\title{
Recent Advancement in Biological Sciences
}

Edited by

Suman Bhusan Chakraborty

Sourav Mitra

Prasanna Kumar Mondal

Sandeep Poddar

Amiya Bhaumik

Published by :

Lincoln Research and Publications Limited, Australia in collaboration with Lincoln University College, Malaysia

\section{www.lincolnrpl.org $\quad$ www.lucp.net}




\title{
Recent Advancement in Biological Sciences
}

\author{
Edited by \\ Suman Bhusan Chakraborty \\ Associate Professor, Department of Zoology \\ University of Calcutta, India \\ Sourav Mitra \\ Assistant Professor, Department of Physics, \\ Surendranath College, Kolkata, India \\ Prasanna Kumar Mondal \\ Assistant Professor, Department of Physics, \\ Surendranath College, Kolkata, India \\ Sandeep Poddar \\ Deputy Vice Chancellor (Research \& Innovation) \\ Lincoln University College, Malaysia \\ Amiya Bhaumik \\ President \\ Lincoln University College, Malaysia
}

Published by :

Lincoln Research and Publications Limited, Australia in collaboration with Lincoln University College, Malaysia 
Copyright (C2021

Lincoln Research and Publications Limited, Australia and Lincoln University College, Malaysia

All rights reserved

No part of this book can be reproduced or transmitted by any means, electronic or mechanical, including photocopying recording or by any information storage and retrieval system without prior written permission from the publisher.

Published on: $30^{\text {th }}$ November 2021

\section{Published by:}

Lincoln Research and Publications Limited

144A, Marsden Road

Ermington, Sydney

NSW 2115

Australia

Tel.: +61-411497511

E-mail: info@lincolnrpl.org

Web.: www.lincolnrpl.org

in collaboration with

\section{Lincoln University College}

Wisma Lincoln

No. 12-18, Off Jalan, Perbandaran SS 6/12

47301 Petaling Jaya

Selangor Darul Ehsan

Malaysia

Tel.: +603-7806 3478

Fax: +603-7806 3479

Toll Free: 1-300-880-111

E-mail: lucp@lincoln.edu.my

Web.: www.lucp.net

ISBN: 978-0-6488798-6-2

eISBN 978-967-2819-02-8

doi:10.31674/book.2021.rabs

Price: AUD 50 


\section{Editors}

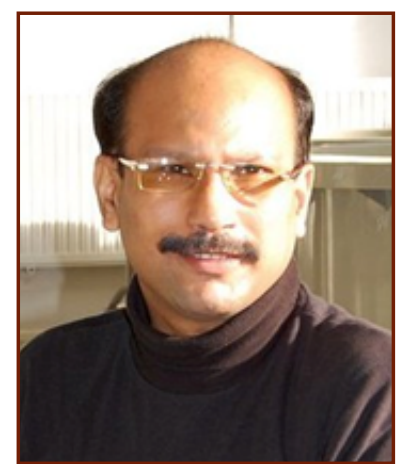

\section{Suman Bhusan Chakraborty}

Associate Professor, Department of Zoology

University of Calcutta, India

Dr. Suman Bhusan Chakraborty is currently an Associate Professor at Department of Zoology, University of Calcutta, India. He received his Ph.D. degree in Zoology from University of Calcutta and completed his postdoctoral research work from Kaposvar University, Hungary. Prior to joining the university, he served Serampore College, West Bengal, India for twelve years as an Assistant Professor. He is the recipient of the Gold Medal in Zoology 2000 from University of Calcutta for securing first class first position in M.Sc. He is engaged in research work on reproductive endocrinology and stress physiology in fish models. He has presented his research work in various international conferences and obtained research funding from different national funding agencies. He has received several national awards for his achievements in research related to aquaculture and fish biology. He has more than 50 research papers published in peer-reviewed scientific journals.

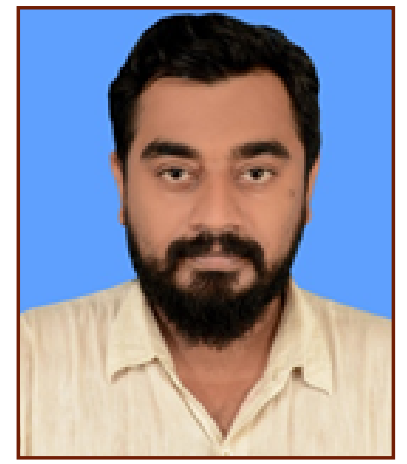

\section{Sourav Mitra}

Assistant Professor, Department of Physics

Surendranath College, Kolkata, India

Dr. Sourav Mitra obtained his Ph.D. in Astrophysics from Harish-Chandra Research Institute, Allahabad. After that he pursued Post-Doctoral research in University of Western Cape, Cape Town, SA and Inter-University Centre for Astronomy and Astrophysics (IUCAA), Pune, India for 4 years. He is currently working as Assistant Professor at Department of physics of Surendranath College, Kolkata. Dr. Mitra has published more than 20 papers in peer-reviewed international journals. His area of research includes Cosmological reionization, Galaxy formation and Dark Matter. He got Young Astronomer of the Year 2013 Award by the Astronomical Society of India and the INSPIRE Faculty Award by Department of Science \& Technology (DST), Government of India in 2017.

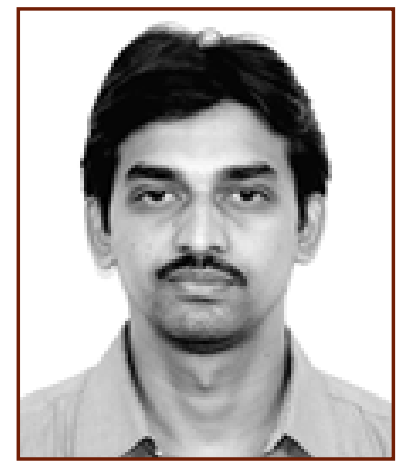

\section{Prasanna Kumar Mondal}

Assistant Professor, Department of Physics

Surendranath College, Kolkata, India

Dr. Prasanna Kumar Mondal, presently working as Assistant Professor in the Department of Physics, Surendranath College, Kolkata, India. He completed his M.Sc. in Physics from University of Calcutta, India. He has done his Ph.D. research work in the Department of Physics of Bose Institute, India and he received his Ph.D. degree from University of Calcutta. He has authored more than 25 research papers in various international peer-reviewed journals. 


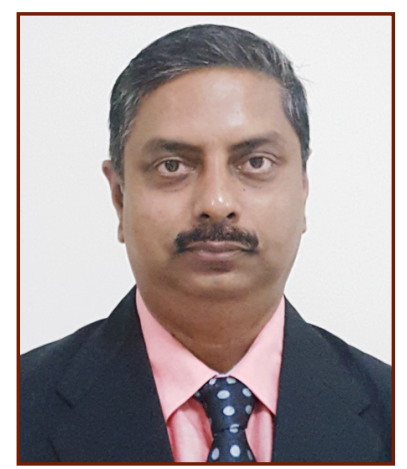

\section{Sandeep Poddar}

Deputy Vice Chancellor (Research \& Innovation)

Lincoln University College, Malaysia

Prof. Dr. Sandeep Poddar, presently the Deputy Vice Chancellor (Research \& Innovation) of Lincoln University College, Malaysia. He also served as Senior Research Director and Executive Editor(Publications), Lincoln University College, Adjunct Faculty (Honorary), Bharat Center Canada. He has graduated from University of Calcutta in 1993 with Honours in Zoology, he has obtained Post Graduate Diploma in Dietetics from All India Institute of Hygiene and Public Health 1995, Master of Science in Zoology with specialization in Biochemical Genetics from Dayalbagh Educational Institute 1998 with distinction. In addition to this he also obtained Master of Business Administration (MBA) from Lincoln University College in 2021. He has completed Ph.D. in Zoology from Vivekananda Institute of Medical Sciences on Cytotoxicity in 2004. After completing $\mathrm{Ph}$.D. he pursued Post Doctoral Research in different projects on Hemoglobinopathies and Oral Cancer mutation. He is serving as reviewer of several International Journals. He has published several research papers, organized international conferences, and edited books in Malaysia, Australia and India. Dr. Sandeep is founder Assistant Secretary of Dr. Tarak Nath Podder Memorial Foundation, Kolkata, India.

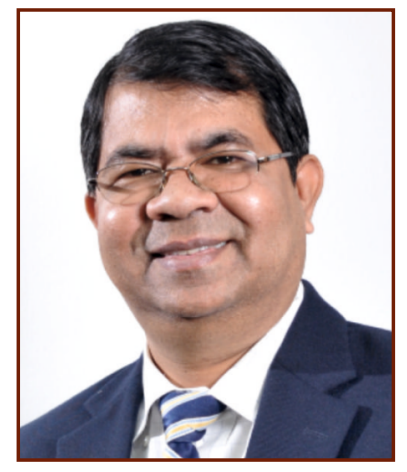

\section{Amiya Bhaumik}

President

Lincoln University College, Malaysia

Dr. Amiya Bhaumik is the Founder and Former Vice-Chancellor of Lincoln University College. He is purely from the field of education. Dr. Bhaumik is Executive Vice President of the International Education Consulting Group, St. Louis, USA since 1999. Dr. Amiya Bhaumik was Research Fellow of UNESCO, Paris. During this tenure, Dr. Bhaumik has traveled extensively to Europe, Africa, Asia and Latin America. He has served as Professor of Business Administration in University of Lucknow, India and in University of Malaya and many other places. Dr. Amiya Bhaumik is a very dynamic personality. He has authored numerous book chapters and has huge number of publication in many national and international journals. He has also edited several books. 
Contents

Pages

Editorial

i-ii

Foreword

An Updated Review on Pharmacological Properties of Ginger (Zingiber officinale Roscoe)

Mitali Paul, Sayan Kumar Saha, Ipshita Bhanja, Dipasree Roychowdhury

Biotechnological Applications of Phototrophic Purple Nonsulfur Bacteria

Anannya Banerjee, Soumita Debnath, Trishita Mukherjee, Baishali Pandit

\section{A Short Review on Gastric Cancer}

Sneha Dey, Biswanath Dari, Subhrajit Kundu, Lalita Das

Bio-Inks for 3D Bioprinting: A Scientific Analysis of Two Decades of Progress, Challenges and Future Prospects

Sritama Chatterjee, Sayak Pan, Suchandra Chatterjee

3D Bioprinting: Various Methods and Techniques for Tissue and Organ Regeneration

Sayak Pan, Sritama Chaterjee, Suchandra Chatterjee

An Overview on The Threats and Conservation Strategies of Royal Bengal Tiger

(Panthera tigris tigris) in Sundarban, India

Aniket Chatterjee, Disha Chatterjee, Biswajit Santra, Adity Sarbajna

Impacts of Covid-19 Pandemic

Aslam khan, Titli Mistry, Tarikul Islam Golder

Inorganic Nanoparticles

Atanu Ganguly, Anirudha Bhattacharjee, Shourya Ghosh, Apurba Biswas

Ocimum sanctum L., Phytochemistry And Effect on Cancer Cells

Ranjan Shaw, Bidisha Sarkar, Rakesh Mondol, Purbasha Sadhukhan, MD. Ahmed Karim, Kousik Ghosh

Induced Pluripotent Stem Cells (iPSCs): A Highway Under Construction

Kajal Das, Shreya Deb, Sagar Kumar Saha, Subhadipa Majumder

Polycystic Ovarian Syndrome Thieving Womanhood

Arun Jana, Sumona Naskar, Subhadra Roy

A Review on Curcumin and its Derivatives as Anticancer Agents

88-96

Subhadeep Sengupta, Suman Mahato, Jagannath Dalui, Lalita Das 

The study of life and living organisms, their life cycles, adaptability, and environment is referred to as biological sciences. Bioinformatics, genomics, metagenomics, proteomics, enzymology, agriculture and marine microbiology, bioremediation, medicinal chemistry, and nanotechnology are among the fast-increasing biological sciences covered in "RecentAdvances in Biological Sciences."

Zingiber officinale Roscoe (commonly known as ginger) is a perennial herb belonging to the aromatic family Zingiberaceae. In addition to its regular use in culinary practices worldwide, ginger has been used as a medicinal herb in Asian culture for more than 5000 years. The rhizome of ginger is well known to be utilized in traditional medicines for varied diseases including-common cold, nausea, migraines, inflammation, dysmenorrhoea, obesity, cholesterol, chronic obstructive pulmonary disease and as an expectorant to mention a few.

The physiological and biochemical characteristics of phototrophic purple nonsulfur bacteria (PPNSB) are unique. These microbes may grow and thrive in a variety of different environments. Purple non sulphur bacteria are the most diverse and versatile of all photosynthetic bacteria with vast potentials for applications. Hence, in view of their abilities to produce biohydrogen, bioplastic, bioherbicides, phytohormones and industrially important enzymes they can be exploited for industrial production of these products.

Gastrointestinal cancer is another name for stomach cancer. Stomach cancer develops when healthy cells in the stomach become aberrant and proliferate uncontrollably. A cancerous tumour can develop and spread to other regions of the body. Stomach Cancer can start in any part of the stomach. The stomach being affected by cancer, has the potential to spread to the adjacent lymph nodes and other body regions. Stomach cancer can damage organs such as the liver, bones, lungs, and women's ovaries.

The overall interest for organ replacement and tissue regeneration is expanding consistently with time. The advancement in tissue engineering and regenerative drug production utilizing 3D bioprinting has succeeded to regenerate broken organs and tissues into perfect ones. The essential material for this type of printing is Bio Ink, which is a must for creating practical organs and tissue structures. Bio fabrication is a rising field that includes formulations of tissue structures with a definite planning. Typical bio fabrication techniques include various steps like desiccation, electrospinning, microengineering etc.

3D bioprinting could be a spearheading innovation permitting manufacture of biomimetic, multiscale, multi-cellular tissues with incredibly confounded tissue microenvironment, complex structure, structure-function relationship, and tissue-explicit integrative and mechanical nonuniformity. 3D bioprinting has acquired energy with the generation of valuable 3D human constructs imitating native tissues/organs.

The largest contiguous lagoon wetland in the world is Sundarbans. The magnificent endangered species among the animals on land is Royal Bengal Tiger. As per Red data book the species is highly threatened. The effect of ecological degradation of Sundarbans in the present status of Panthera tigris is clearly reflected by its number in its natural number. It is difficult to conserve tigers embedded within densely populated human dominated landscapes as it causes conflict between human and tiger. The main cause of this conflict is the livestock grazing and human intrusion into tiger habitat and poor husbandry. So, protection of tiger can be performed by providing them adequate grazing area, abundance of prey, and proper monitoring of their lives. Wide population size and adjacent habitat of Sundarbans is apparently penetrable by tigers. So, the Sundarbans is very much valuable for tiger conservation.

The Corona infection or COVID-19 is a sickness dependent on an obscure infection. It appears to be that it began in China and has broadly spread in practically all nations on the planet. This pandemic circumstance is one of the broadly spread infections in late history. The proposed future action plan or COVID-19 recovery action plan mainly focuses on the socioeconomic, environment and political spheres and not on the medical and technical spheres. 
Inorganic nanomaterials, including gold nanoparticles, silver nanoparticles, iron nanoparticles, silica nanoparticles, and magnetic nanoparticles, are non-toxic, which tend to mix, and are highly stable compared to organic materials. In the field of modern material science, inorganic nanoparticles are being developed as novel drug delivery systems because of their own physical properties, which include size-sensitive optical, magnetic, electronic, and catalytic properties. These nanoparticles have many physical properties, including high stability, high surface area, tenable compositions, and abundant physicochemical multifunctionality.

Herbs and other plant products have been used as medicine across the world since the beginning of time. Ayurveda, which focuses on employing natural plant products as therapeutic agents, was the principal way of treating ailments in ancient India. The sacred plant Ocimum sanctum, often known as 'Tulsi,' may be found all over India. It is not only revered and feared, but it is also utilised as a home cure for a variety of respiratory problems. It is a cytotoxic drug that kills cancer cells in the mouth. The presence of phytochemical substances, which are abundant in Tulsi leaves, is responsible for this action. The herb which is abundantly grown in India can be used for its anticancer properties.

The ability to produce induced pluripotent stem cells (iPSC) opens the prospect of generating practically all cell types in the human body. A new set of strong tools for life science has become available when modern gene editing techniques like CRISPR/CAS are combined. Scientific domains such as genotype and cell type-specific pharmacology, disease modelling, stem cell biology, and developmental biology have all seen significant advancements and new faces.

PCOS (polycystic ovarian syndrome) is a hormonal condition that affects many women of reproductive age. The disorder polycystic ovarian syndrome (PCOS) alters a woman's hormone levels. This hormonal imbalance causes them to miss menstrual periods, making it difficult for them to conceive. Polycystic ovarian syndrome is a deeply stigmatising condition with far reaching implications for all women. The basic treatment of controlling this condition is by changing the lifestyle and taking specific medication.

Understanding biological systems in all their complexity while protecting and using biological organizations in a sustainable manner is the great problem and opportunity for biological science as we move into the twenty-first century. At all levels, from the molecular to the cellular, the system, the organism, and the community of organisms, there is a compelling need to take use of and develop new analytic tools. The technology advances discussed in this book will be cutting-edge in some circumstances.

The editors would like to thank all the authors for their contribution in such varied topics that will help to create awareness in various aspect of life. Editors are also thankful to Principal, Surendranath College, Kolkata, India, Management of Lincoln University College, Malaysia and Lincoln Research \& Publishing Limited, Australia for giving us the necessary permission to publish this book. We are also thankful to Department of Biotechnology, Government of India for providing the financial assistance. This book provides the latest technologies, advanced methods, and unexploited research areas involved in varied fields of biological science research. Each chapter is written by experts in their respective fields of biosciences and comprises of future advancements in life science research.

Suman Bhusan Chakraborty

Sourav Mitra

Prasanna Kumar Mondal

Sandeep Poddar

Amiya Bhaumik 


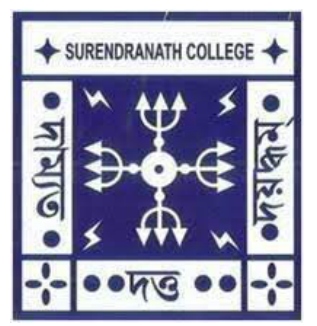

\title{
Surendranath College
}

24/2, Mahatma Gandhi Road, Kolkata - 700 009, India

Phone: (033) 2350 2864, 2354 3876, Fax: (033) 23502864

E-mail: snlcollege@gmail.com, Web.: www.snlawcollege.ac.in

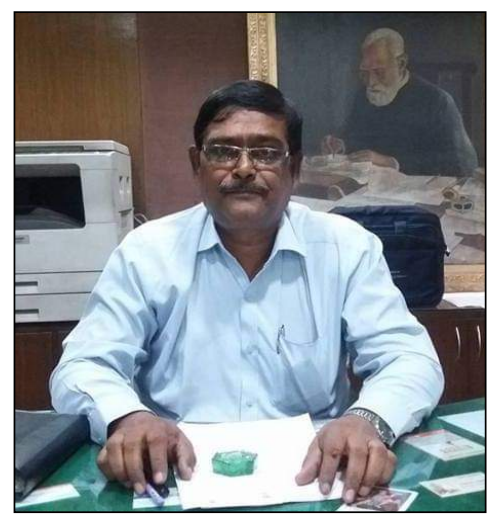

\author{
Dr. Indranil Kar \\ M.Sc., Ph.D. \\ Principal \\ Surendranath College \\ Kolkata, India
}

\section{FOREWORD}

It is my great pleasure to introduce this book titled as "Recent Advancement in Biological Sciences," a collection of review articles written by the undergraduate students of the four DBT STAR departments of Surendranath College, Kolkata, covering a wide range of contemporary themes on current advances in various areas of biological sciences, under able mentorship of their faculty members as mentors.

The volume consists of twelve chapters and pursues specific objectives while complementing the existing literature on different areas of biological sciences. It explores the scope of future developement in various related emerging fields and analyses the challenges in doing so. I extend special thanks and underline my deepest appreciation to the publisher for publishing this issue, Department of Biotechnology, Government of India for providing the financial assistance and all the editors of this book for their real hard work and sincere effort in bringing out this wonderful volume.

While I hope this collection will have particular appeal to all the new young readers in this field, I am confident that the volume will raise interest among our regular readership of scholars and practitioners elsewhere as well, thanks to the relevance and diversity of contributions compiled here in this volume.

Happy reading to all!

Dr. Indranil Kar

Principal

Surendranath College

Kolkata, India 



\title{
An Updated Review on Pharmacological Properties of Ginger (Zingiber Officinale Roscoe)
}

\author{
Mitali Paul, Sayan Kumar Saha, Ipshita Bhanja, Dipasree Roychowdhury* \\ Department of Botany, Surendranath College, Kolkata, India \\ ${ }^{*}$ Corresponding Author's Email: dipasree.roychowdhury@yahoo.com
}

\begin{abstract}
Zingiber officinale Roscoe (commonly known as ginger) is a perennial herb belonging to the aromatic family Zingiberaceae. In addition to its regular use in culinary practices worldwide, ginger has been used as a medicinal herb in Asian culture for more than 5000 years. The rhizome of ginger is well known to be utilized in traditional medicines for varied diseases including-common cold, nausea, migraines, inflammation, dysmenorrhoea, obesity, cholesterol, chronic obstructive pulmonary disease and as an expectorant to mention a few. The medicinal properties of ginger are particularly attributed to its active phytochemicals, namely, 6-gingerol, 6-shogaol, zingerone and other phenolics and flavonoids. Over the last few decades, extensive investigations conducted on $Z$. officinale revealed plethora of pharmacological properties present in the rhizome of the plant like, anti-oxidant, antidiabetic, anti-cancerous, anti-obesity, anti-microbial, anti-inflammatory, cardio-protective, etc. This review article aims in providing an updated status on pharmacological properties reported in rhizomes of $Z$. officinale based on scientific literatures and its future in medicine.
\end{abstract}

Keywords: Zingiber Officinale Roscoe; Ginger; Medicinal Properties; Pharmacological Properties

\section{INTRODUCTION}

According to World Health Organization (WHO), about $80 \%$ of the world's populations still rely mainly on traditional therapies which involve the utilization of plant extracts or their active substances (World Health Organization, 2003). The use of 'natural' or 'alternate herbal' medication has increased markedly over the previous years. The utilization of plants to treat various diseases in India dates back to the days of Rig Veda (3500 to 1800 B.C.). Later, the monumental Ayurvedic works like Charaksamhita and Sushrutasamhita followed by other Ayurveda and Siddha treatises have incorporated nearly 700 plant drugs getting into several medicinal preparations used in the management of health care. In reality these systems are in practice even today in remote areas of our country for hundreds of years (Mukherjee et al., 2017).

The medicinal use of ginger is documented in India and its neighbouring countries for more than 5000 years as one of the most versatile medicinal plants (Silver, 2007). Zingiber officinale, commonly referred to as ginger, belonging to the Zingiber aceae is a perennial herb (The Plant List, 2013).English botanist William Roscoe (1753-1831) coined the name Zingiber officinale in 1807. The name 'Zingiber' was derived from a Sanskrit word 'Shrungver', where 'Shrung' means 'horn' and 'ver' means 'body'due to the horn shaped protrusions present on the rhizome prevalent in this genus. This vegetative propagating plant is distributed throughout tropical Asia and also commercially cultivated in other parts of the world including Mexico. In India, it is widely cultivated including the states of Uttar Pradesh, West Bengal, Andhra Pradesh, Maharashtra and Kerala, for its extensive use as spice and/or condiment as well as its wide application in traditional system of herbal medicines- Ayurveda, Unani, Sidhha and Chinese medicine (Kritikar, Basu \& Blatter, 1993; Khare, 2008). In traditional Chinese medicine, as well as modern China, ginger is used in about half of all herbal prescriptions. The traditional use of this aromatic rhizome in medicine includes treatment of common cold, nausea, migraines, inflammation, dysmenorrhoea, obesity, cholesterol, chronic obstructive pulmonary disease and as an expectorant to 
mention a few. In traditional medicine it is widely used as an aphrodisiac and to cure hormonal imbalance (Sharma, 2019). The medicinal properties of ginger are particularly attributed to its active phytochemicals, namely, 6-gingerol, its dehydrated derivative- 6-shogaol, zingerone and other phenolic and flavonoid compounds. This review article aims in providing an updated status on pharmacological properties reported in rhizomes of $Z$. officinale and its active compounds based on scientific literatures and its future in medicine.

\section{REVIEW OF LITERATURE}

\section{Pharmacological Properties of Zingiber officinale}

Zingiber officinale (ginger) extracts and its important secondary metabolites, namely- gingerol, shogaol, zingerone, etc., are reported to have plethora of pharmacological properties, for example, anti-oxidant and anti-inflammatory (Yeh et al., 2014; Oliveira et al., 2020; Venkateswaran et al., 2021), anti-obesity (Wang et al., 2017; Sampath et al., 2021), anti-diabetic (Al-Amin et al., 2006; Venkateswaran et al., 2021), anti-clotting (Thomson et al., 2002; Saputri, Nabila \& Mun'im, 2017), antiviral (San Chang et al., 2013; Wang et al., 2020 a; Ahkam et al., 2020 \& Haridas et al., 2021), analgesic (Kravchenko et al., 2019) and many more. The recent advancements in the field of each therapeutic properties of ginger and its phytochemicals are discussed in detail in the following section.

\section{DISCUSSION}

\section{Anti-diabetic property}

Diabetes mellitus is a common metabolic disorder, characterised by inability of pancreas to produce insulin or the body's inability to utilise it after being produced. Z. officinale has shown to be effective against both Type-1 and Type-2 diabetes mellitus (T1DM and T2DM) and in improving diabetes related complications in pre-clinical studies with animal models and in vitro cell cultures(Al-Amin et al., 2006; Akash et al., 2015; Nam et al., 2020; Venkateswaran et al., 2021). Some reports of clinical trials are also reported for effectiveness of ginger in controlling blood sugar in humans (Hajimoosayi et al., 2020; Nunes Carvalho et al., 2021).

Treatment using Z. officinale showed a significant rise in insulin levels and reduction in fasting glucose levels in Streptozotocin-induced diabetic rats (Akhani, Vishwakarma \& Goyal, 2004; Al-Amin et al., 2006). In addition, raw ginger feeding showed effective in reversing the diabetic proteinuria in these animal models. Ginger works on T2DM by increasing the insulin synthesis or its sensitivity and providing protection to the pancreatic cells and ultimately increasing the glucose uptake by the tissues. It also decreases the diabetes related complications by reducing fat accumulation, decreasing oxidative stress, protects one against nephropathy and diabetic cataracts due to its antioxidant effects (Akash et al., 2015). Nam et al., (2020) observed significant reduction of hyperglycemia in diabetic mouse models treated with steamed ginger extracts and suggested the mode of action by stimulating insulin secretion by closing the $\mathrm{K}_{\text {ATP }}$ channels of the pancreatic beta cells. In a recent study, ginger extracts, precisely gingerol derivatives and shogaol showed increased uptake of glucose in cell culture system. Further, the antidiabetic potency could be directly correlated with the polyphenol rich varieties of the herb (Venkateswaran et al., 2021). Hence, the reports suggest, ginger administration might have a potential therapeutic role in managing the effects of diabetic complications in human.

Clinical trial with 70 women with gestational diabetes, which is very common complication during pregnancy,showed oral administration of ginger tablets significantly decreased fasting blood sugar levels and improved insulin levels compared to placebo controls in a recent study conducted in Iran. However, the ginger intervention did not show significant decrease in the blood sugar level after 2 hours of meal (Hajimoosayi et al., 2020). In another randomised clinical trial with T2DM patients from age group 18-80, the 4-week intervention programme with $1.2 \mathrm{~g}$ ginger per day, showed to reduce anthropometric measures and lipid levels in the population compared to placebo controls, however, no significant effect was observed in the glycemic levels (Nunes Carvalho et al., 2021). Therefore, detailed studies are further required to prove the effectiveness of this herb in treating diabetes in 
human, although numerous studies support its proficiency in pre-clinical studies.

\section{Anti-obesity properties}

Numerous natural herbs are proven as safe anti-obesity resources. Ginger, one of the most widely consumed spices, has shown beneficial effects against obesity and related metabolic disorders. A large number of systematic reviews and meta-analyses on the potential of ginger and its active compounds in controlling obesity including diabetes related body mass increase are available recently (Wang et al., 2017; Ebrahimzadeh Attari et al., 2018 \& Tramontin et al., 2020). The studies on affectivity of ginger on obesity include in vitro studies, observations in animal models as well as more than 20 reportson human clinical trials.

In pre-clinical studies, high fat diet fed mice showed significant decrease in weight gain, lipid accumulation and reduction in adepocyte size in the test groups administered with ginger essential oils compared to control (Lai et al., 2016; Wang et al., 2019 \& Pragasam et al., 2021). 6-gingerol and aqueous extracts of ginger seemed to be the most effective for treatment of obesity in the animal models. In addition, 6-gingerol suppressed the activity of amylase and pancreatic lipase, leading to a reduction of plasma and tissue lipids. The anti-obesity effects of ginger can be associated with its antiinflammatory properties, as ginger extract and 6-gingerol can notably down regulate mRNA levels of interleukin- 6 and tumor necrosis factor in adipose tissue along with modifying gut microbial of high fat diet fed male rats (Wang et al., 2019).

Clinical trials with ginger supplementation have showed interesting effects in reduction of fat content and anthropometric measures in studied groups. Dietary intake of ginger showed advantageous effect on weight loss and some obesity-associated parameters in overweight and obese adults (Wang et al., 2017). In addition to decrease in body weight and fat accumulation, decrease in hip circumference is also noted in clinical trial with high dose of ginger (Honarvar et al., 2019). The mode of action is presumed to be via controlling appetite of the test subjects by serotonin modulation, regulating satiety, increasing body temperature, increase in metabolism rate, increasing lipid breakdown, decreasing lipogenesis, increasing fatty acid oxidation, regulating absorption of fat in intestine and by blocking adipose tissue generation (Wang et al., 2017 \& Tramontin et al., 2020).

In comparison to the large number of studies in animal model, human trails are limited in number and most of the studies with desired results in animal models are based on high dose of ginger extracts. Therefore, a lot of more clinical trials are necessary before implementing ginger as a potent solution to obesity in humans.

\section{Anti bacterial properties}

Anti-bacterial properties of ginger have been proved in large number of studies against a wide range of bacteria, both Gram positive and Gram negative. Ginger extracts showed antibacterial properties against Gram positive bacteria including- Staphylococcus aureus, Staphylococcus epidermidis, Enterococcus faecalis, Bacillus cereus, B. subtilis, B. megaterium, Streptococcus faecalis, etc. $Z$. officinale is also reported to be similarly effective against Gram negative bacteria, namely, Escherichia coli, Klebsiella pneumoniae, Salmonella typhi, Salmonella typhimurium, Pseudomonas aeruginosa and Proteus sp. (Akintobi et al., 2013; Ganjre et al., 2015; Lucky, Igbinosa \& Jonahan, 2017; Abdalla \& Abdallah, 2018; Mohammed et al., 2019 and Wang et al., 2020a). Both aqueous and ethanol extracts of ginger showed effective bactericidal properties against the tested bacterial strains in these studies. Ginger roots extract at high concentration have strong antibacterial activity against pathogenic bacteria S. aureus, E. coli and K. pneumonae in a dose dependent manner. (Mohammed et al., 2019). Wang et al., (2020) not only demonstrated the potency of ginger in controlling $S$. aureus and $E$. coli, the team also elucidated the mechanism of action suggesting, that, the main active principles responsible for the bactericidal property are zingiberene and $\alpha$-curcumene, which kills the tested organisms by disruption of bacterial cell membrane (Wang et al., 2020b). 


\section{Antiviral properties}

Ginger extracts have been reported to have impressive antiviral properties against different pathogenic viruses like Human Respiratory Syncytial Virus (San Chang et al., 2013), Dengue virus (Sharma, Klinzing \& Ramos, 2015), Hepatitis C virus (Abdel-Moneim et al., 2013), Chikunguniya Virus (Kaushik et al., 2020) and Influenza A Virus (Wang et al., 2020b) to name a few. Haridas et al., (2021) recently reported in silico studies suggesting possible potency of ginger in controlling viral load and shedding of SARS-CoV-2 in nasal passage due to its affinity for the viral spike protein as well as angiotensin-converting enzyme 2 (ACE-2). Similar potential of bioactive compounds of ginger against SARS-CoV-2 infection was suggested earlier by Ahkam et al., (2020).

Some studies suggested only fresh ginger to be effective against certain viruses in blocking the viral attachment and internalization (San Chang et al., 2013). While most of the studies were conducted on cell culture systems to determine the anti-viral activity of different extracts of ginger. In cell culture studies, ginger extracts showed impressive activity against Chikunguniya Virus (Kaushik et al., 2020) while, Gingerone A suppressed viral replication of different subtypes of Influenza A Virus (Wang et al., $2020 \mathrm{~b})$. There are also reports of beneficial effects of ethanolic ginger extracts in combination with Nigella seeds for treatment of patients infected with Hepatitis $\mathrm{C}$ virus in clinical trials in Egypt. The plant extracts administered in combination performed better than individual applications in not only reduction of the viral load in patients but also improved their liver function parameters indicating therapeutic role of the herbs (Abdel-Moneim et al., 2013).

\section{Anti-fungal properties}

Zingiber officinale not only scores high in protection against a variety of bacteria and viruses, it is also effective against fungal pathogens. Antifungal properties of aqueous and ethanolic extracts of ginger are well recorded against fungal strains namely, Aspergillus flavus and Candida albicans (Lucky, Igbinosa \& Jonahan, 2017). In the studies reported, $A$. flavus was found to be the most resilient strain demanding high concentration of ethanolic ginger extracts to show antifungal effect. However, $C$. albicans isolate was easily susceptible to both aqueous and alcoholic extracts of the herb.

\section{Anti-oxidant properties}

Anti-oxidant property of ginger is reported in a large number of studies since 1990s. While earlier studies mainly dealt with anti-oxidant potency of different ginger extracts, recent studies are mainly concentrated on the free radical scavenging properties of specific bioactive phytochemicals of $Z$. officinale, in addition to crude extract studies. Methanolic followed by ethanolic extracts of ginger rhizomes are reported to be more potent than alcoholic extracts in battling against free radicals (PR \& Prakash, 2010; Yeh et al., 2014). Interestingly, some studies suggest high anti-oxidant capacity of leaf extracts of ginger in addition to young rhizomes and the potency is directly correlated with the total phenolic content of the plant (Ghasemzadeh, Jaafar \& Rahmat, 2010). Due to high antioxidant activity of ginger and its extracts, it is suggested to be effective in preventing and/or curing a number of diseases involving inflammation (Kulkarni \& Deshpande, 2016). Oliveira et al., (2020) further suggested that organic farmed ginger is more effective in its anti-oxidant properties as it is rich in 6gingerol in addition to total fibre and protein content.

\section{Anti-inflammatory properties}

Inflammation at site of injury or infection is caused by rush of blood with increased white blood cells to fight against invaders. It can be short lived (for few hours) or chronic (long lasting) in nature. Diseases like rheumatoid arthritis, gouty arthritis and other autoimmune diseases cause chronic inflammation. Ginger is well known for its anti-inflammatory properties over the ages in traditional medicine and also proved scientifically by different studies over the decades (Kulkarni \& Deshpande, 2016; Kravchenko et al., 2019; Shahzad, Anderson, \& Najafzadeh, 2020; Venkateswaran et al., 2021). Transdermal administration of ginger extracts is reported to have significant anti-inflammatory effect in mice model (Kravchenko et al., 2019). Shagaol and gingerol are the main phytochemicals responsible for the anti- 
inflammatory properties of Zingiber officinale, which acts by preventing the macrophages and neutrophil activation and migration of the leukocytes at the infected area (Shahzad, Anderson, \& Najafzadeh, 2020). Different randomised clinical trials on human patients for efficacy of ginger and it compounds as anti-inflammatory drug is reported (Kulkarni \& Deshpande, 2016; Mozaffari-Khosravi et al., 2016). Patients with tuberculosis administered $3 \mathrm{~g}$ of ginger extracts daily for a month showed significant improvement in reduction of inflammatory cytokines compared to placebo controls (Kulkarni \& Deshpande, 2016). Similar reduction of cytokines after 3 months period of oral administration of ginger was reported for osteoarthritis patients in a clinical trial $(n=120)$ compared to placebo controls (Mozaffari-Khosravi et al., 2016).

\section{Neuro-protective activity}

Alzheimer's and Parkinson disease are some of the most common neurodegenerative diseases which cause irreversible damage to the brain cells. Ginger and its important secondary metabolites can be promising neuro-protective agents as observed in different studies in animal models (Hussein et al., 2017; Choi et al., 2017). Daily administration of ginger extracts for 30 days caused significant reversal of neurotoxicity in monosodium glutamate treated male rat models (Hussein et al., 2017). The protective activity of ginger is suggested to be due to antagonistic role of ginger on monosodium glutamate. Similar neuro-protective potential of 6-paradol rich fermented ginger extracts was evident in amyloid-beta induced neurotoxicity in rat subjects (Choi et al., 2017).

\section{Analgesic properties}

Ginger and its bioactive compounds have proved to be effective to ameliorate a wide range of pain, including, migraine, toothache, stomach ache, joint pains due to osteoarthritis and rheumatoid arthritis to name a few (Kravchenko et al., 2019; Chen \& Cai, 2021). In migraine, headache is a recurring problem, often reported once in two weeks requiring bed rest, thus, to avoid long term side effects of regular drugs, alternative medicine has gained importance in recent years. Chen and Cai (2021) recently published a detailed meta-analysis on the potency of $Z$. officinale in treatment of migraine patients based on randomised control clinical trials reported worldwide. The study concludes ginger to be a safe herbal medicine to be used against migraine patients reducing pain within 2 hours of administration without any adverse effects. In osteoarthritis patients also, ginger showed impressive power to reduce pain due to its anti-inflammatory properties (Mozaffari-Khosravi et al., 2016).

\section{Anti-thrombotic properties}

Zingiber officinale has been used for ages in Chinese traditional medicine as blood thinning agent and promoting better blood circulation. In rat models different studies have proved the effectiveness of ginger for its anti-blood clotting properties either alone or in combination with other plant extracts like wood of Caesalpinia sappan (Thomson et al., 2002; Saputri, Nabila \& Mun'im, 2017). It is particularly effective when administered in high doses $(500 \mathrm{mg} / \mathrm{kg}$ body weight) both orally as well as intraperitoneal in the animal models for 30 days. Due to the anti-thrombotic properties of ginger, it must be carefully taken by patients with blood clotting disorder or those who are already taking some blood thinning drugs.

\section{Effect on blood pressure}

Due to the blood thinning effect as well as promoting improved blood circulation, Z. officinale consumption is also recommended for lowering or controlling blood pressure in traditional medicine. It is used widely for treatment of hypertension in the herbal treatment practices. Reports suggest, oral administration of ginger results lowering of blood pressure in human trials in dose dependent manner after 2-4 hours (Ojulari et al., 2014). The mode of action is suggested to be via blockage of voltage dependent calcium channel by the active phyto-constituents of ginger as elucidated in animal models (Ghayur \& Gilani, 2005). The main bioactive compound of ginger responsible for this blood pressure lowering effect is due to 6-gingerol, which inhibits angiotensin II type 1 receptor activation due to its antagonistic property and thus helps in lowering blood pressure and improving overall cardiovascular 
heath of an individual (Liu et al., 2013).

\section{Cardio-protective properties}

Ginger not only promotes blood circulation and aids in controlling blood pressure, but it is also effective in strengthening cardiovascular status. In a recent article, Li et al., (2021) elaborated the wide range of cardio protective activities ginger is capable of, based on the extensive literature survey. The combined properties of $Z$. officinale rhizomes, including, anti-inflammatory property, anti-oxidant property, ability to reduce lipid accumulation and promote lipid metabolism, thus lowering blood cholesterol, inhibition of platelet aggregation in blood vessels, increasing nitric oxide synthesis and blocking voltage dependent calcium channels, etc are some of the important properties that helps in maintain a good cardiovascular health in individuals ( $\mathrm{Li}$ et al., 2021; Ma et al., 2021). The major phytochemical 6-gingerol is again believed to be the key role player for the cardio-protective role of ginger (Ma et al., 2021).

\section{Anti-cancerous properties}

Ginger and its secondary metabolites have gained much importance in current study due to its antiproliferative properties and are believed to be a potential treatment against wide range of cancers, including, breast cancer, cervical cancer, gastrointestinal cancer, blood cancer, liver cancer, colorectal cancer and many more. Hamza et al., (2021) studied effect of ginger on diethylnitrosamine induced liver cancer in rats. The study showed impressive protective power of ginger against liver cancer due to its anti-oxidant and anti-inflammatory properties, ultimately inhibiting uncontrolled cell proliferation and promoting of apoptosis in the test subjects (Hamza et al., 2021). Zingiber officinale is the most common herbal drug used against colon cancer and research proved six bioactive compounds of fresh ginger including major phenol, 6-gingerol, to interact with multiple pathways including signalling pathways involved in cancer progression to control colon cancer (Zhang et al., 2021).

\section{Hepato-protective properties}

Abdel-Azeem et al., (2013) observed ginger to protect liver against acetaminophen induced acute liver toxicity in mice subjects comparable to vitamin E. Similar protective nature is reported in diethylnitrosamine induced toxicity in rats with dry ginger powder administration for 2 months (Fahmi et al., 2019). Hepato-protective properties of $Z$. officinale is reported against a number drug induced liver toxicity, alcoholic liver damage and non-alcoholic hepatic ailments in animal models, however clinical trials with human subjects are yet to be reported.

\section{Protection against renal damage}

Nephro-toxicity is one of the main side effects of a number of antibiotics and drugs in long term usage. The anti-oxidant and anti-inflammatory properties of ginger help in protecting the kidney against different toxic chemicals. Nephro-protective property of Zingiber officinale is well established against alcohol induced chronic kidney damage in animal models suggesting regular dietary intake of ginger to have protective function on renal cells (Ramudu et al., 2011). Similar protective property of ginger against gentamycin induced renal damage of rats is reported by Rodrigues et al., (2014). Almatroodi et al., (2021) suggested 6-gingerol to be the main bioactive compound responsible for reducing renal damage in drug induced nephrotoxicity in rats due to its anti-oxidant and anti-inflammatory properties.

\section{Anti-emetic properties}

Anti-emetic drugs are often needed to be prescribed for patients struggling with nausea and vomiting tendencies due to side effects of drugs, chemotherapy, in morning sickness during pregnancy, etc. Ginger has been used in traditional medicine for its ability to reduce the feeling of nausea and vomiting since ages. Modern research shows, 6-gingerol of fresh Zingiber officinale rhizome and 6-shogaol present in dried ginger powder to be the most effective compounds responsible for the anti-emetic properties reported. Ginger biscuits have shown to reduce the morning sickness in women during early pregnancy (Basirat et al., 2009). The herb is shown to be equally effective in treatment of nausea 
and vomiting in breast cancer patients after chemotherapy (Saneei Totmaj et al., 2019). Thus, fresh and dried ginger, both is reported to have effective antiemetic properties which are well proven in human patients.

\section{Radio-protective properties}

Radiation therapy has become an important type of intervention in the treatment of different forms of cancer, which often comes with an array of side effects including damage to normal healthy cells. With the help of appropriate radio-protective agent, proliferation of cancer cells can be controlled more efficiently, as higher dosage of radiation could be administered without the fear of damaging normal cells to certain extent. Baliga et al., (2012) reported a comprehensive summary of the prospects of Zingiber officinale rhizome offering protection against radiation therapy. Extensive survey of literature available on studies with animal models and in vitro cell cultures on radio-protective properties of ginger extracts and its important secondary metabolites indicated promising potential of the herb in protection against gamma radiation therapy. The most effective secondary metabolite responsible for the radio-protection is attributed to zingerone and dehydrozinger one of ginger. In animal models, these phytochemicals effectively prevented test subjects from sickness and mortality due to gamma radiation therapy. Anti-oxidant and anti-inflammatory properties of $Z$. officinale are suggested to be the reason behind its radio-protective action. Ginger extracts have also shown to protect cells from gamma radiation induced chromosomal abnormalities, micronuclei formation and DNA damage in bone marrow cells and sperm cells of the subjects (Abd El-Monem \& Elwakeel, 2020). Nabil, El-Zainy and Hassan (2021) indicated the anti-oxidant property of ginger to be the most important character in offering protection to test subjects against radiation therapy.

\section{CONCLUSION}

Zingiber officinale Roscoe (Family: Zingiberaceae) or commonly known as ginger is not only used worldwide as a condiment or spice but is used in traditional medicinal practices for a wide range of treatments since ages in Asian culture. Recent research and pre-clinical trials with animal models and in vitro cell culture systems, confirm the rhizomes of ginger and its active bio-compounds to possess a plethora of pharmacological properties as summarised in the Figure 1.

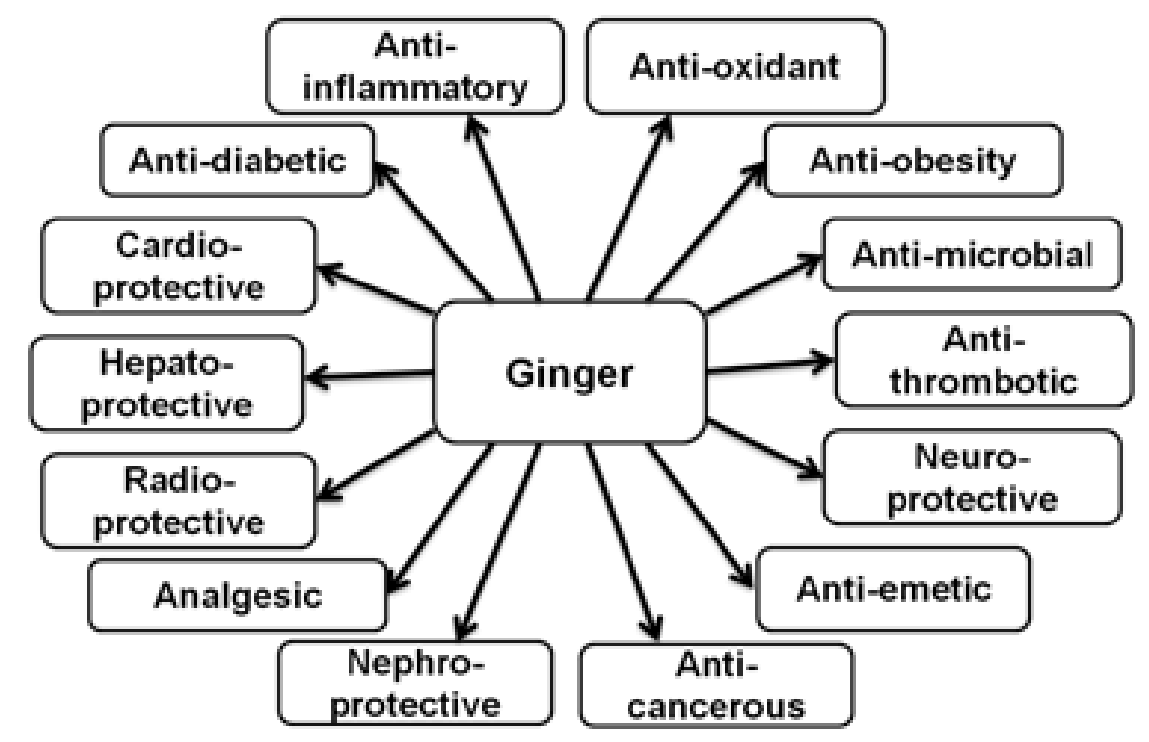

Figure 1: Summary of different pharmacological properties of Zingiber officinale Roscoe

The main phytochemicals responsible for therapeutic properties of ginger are 6-gingerol, 6-shogaol, zingerone and other phenolic and flavonoid compounds. Z. officinale rhizome extracts and its secondary metabolites have promising antimicrobial properties as it was found to be actively working against wide range of bacteria, both Gram +ve and Gram -ve, different types of viruses including in silico studies suggesting to be effective against SARS-CoV-2, as well as effective against different 
fungal strains. Ginger and its bio-active compounds are rich in anti-oxidants and shows impressive anti-inflammatory properties, hence suggested to be effective against different diseases that involve inflammation. The herbal medicine also proved to be effective in protecting test subjects against nephro-toxicity, hepato-toxicity and radiation therapy. Ginger has been used in treatment of diabetes and obesity in traditional medicine since ages and it is not only confirmed by pre-clinical trials but also showed promising results in the limited number of clinical trials on human population available. Human trials are also available for its affectivity against migraine pain which works within 2 hours of administration. Further analgesic properties of ginger are effective against arthritis pain, toothache, stomachache, and many more. The ability of ginger supplements to improve blood circulation, lower blood pressure, lowers lipid accumulation, improve lipid metabolism and ability to reduce in size of adepocytes suggests its potency for offering overall improvement of cardiac health. However, due to the anti-thrombotic property, this herb must be taken carefully for patient already on blood thinners or with blood clotting disorders. Hence, we can conclude ginger to be storehouse of therapeutic properties, but as most pre-clinical trials involved high dosage of ginger extracts, it is extremely important to conduct more clinical trials in future to use it as alternative herbal medicine effectively.

\section{ACKNOWLEDGMENT}

The authors express their sincere thanks to Department of Biotechnology, Government of India for the funding from DBT-STAR College Grant, under which this review project was conducted. The authors are also grateful to the Principal, Surendranath College and the DBT-STAR Coordinator, Surendranath College, for their support and encouragement in implementing this review project.

\section{REFERENCE}

Abd El-Monem, D. D., \& Elwakeel, S. H. B. (2020). Radioprotective efficacy of ginger (Zingiber officinale) extract against gamma-ray radiation-induced genotoxicity in rats. International Journal of Radiation Research, 18(1), 43-55.

Abdalla, W. E., \& Abdallah, E. M. (2018). Antibacterial activity of ginger (Zingiber Officinale Rosc.) Rhizome: a mini review. Int J Pharmacogn Chinese Med, 2, 000142.

Abdel-Azeem, A. S., Hegazy, A. M., Ibrahim, K. S., Farrag, A. R. H., \& El-Sayed, E. M. (2013). Hepatoprotective, antioxidant, and ameliorative effects of ginger (Zingiber officinale Roscoe) and vitamin $E$ in acetaminophen treated rats. Journal of Dietary Supplements, 10(3), 195-209.

Abdel-Moneim, A., Morsy, B. M., Mahmoud, A. M., Abo-Seif, M. A., \& Zanaty, M. I. (2013). Beneficial therapeutic effects of Nigella sativa and/or Zingiber officinale in HCV patients in Egypt. EXCLI Journal, 12, 943.

Ahkam, A. H., Hermanto, F. E., Alamsyah, A., Aliyyah, I. H., \& Fatchiyah, F. (2020). Virtual prediction of antiviral potential of ginger (Zingiber officinale) bioactive compounds against spike and MPro of SARS-CoV2 protein. Berkala Penelitian Hayati, 25(2), 52-57.

Akash, M. S. H., Rehman, K., Tariq, M., \& Chen, S. (2015). Zingiber officinale and type 2 diabetes mellitus: evidence from experimental studies. Critical Reviews ${ }^{\mathrm{TM}}$ in Eukaryotic Gene Expression, 25(2).

Akhani, S. P., Vishwakarma, S. L., \& Goyal, R. K. (2004). Anti-diabetic activity of Zingiber officinale in streptozotocin-induced type I diabetic rats. Journal of Pharmacy and Pharmacology, 56(1), 101-105.

Akintobi, O. A., Onoh, C. C., Ogele, J. O., Idowu, A. A., Ojo, O. V., \& Okonko, I. O. (2013). Antimicrobial activity of Zingiber officinale (ginger) extract against some selected pathogenic bacteria. Nature and Science, 11(1), 7-15.

Al-Amin, Z. M., Thomson, M., Al-Qattan, K. K., Peltonen-Shalaby, R., \& Ali, M. (2006). Anti-diabetic and hypolipidaemic properties of ginger (Zingiber officinale) in streptozotocin-induced diabetic rats. 
British Journal of Nutrition, 96(4), 660-666.

Almatroodi, S. A., Alnuqaydan, A. M., Babiker, A. Y., Almogbel, M. A., Khan, A. A., \& Husain Rahmani, A. (2021). 6-Gingerol, a Bioactive Compound of Ginger Attenuates Renal Damage in StreptozotocinInduced Diabetic Rats by Regulating the Oxidative Stress and Inflammation. Pharmaceutics, 13(3), 317.

Baliga, M. S., Haniadka, R., Pereira, M. M., Thilakchand, K. R., Rao, S., \& Arora, R. (2012). Radioprotective effects of Zingiber officinale Roscoe (ginger): past, present and future. Food \& Function, 3(7), 714-723.

Basirat, Z., Moghadamnia, A., Kashifard, M., \& Sarifi-Razavi, A. (2009). The effect of ginger biscuit on nausea and vomiting in early pregnancy. Acta Medica Iranica, 51-56.

Chen, L., \& Cai, Z. (2021). The efficacy of ginger for the treatment of migraine: A meta-analysis of randomized controlled studies. The American Journal of Emergency Medicine, 46, 567-571.

Choi, J. W., Park, H. Y., Oh, M. S., Yoo, H. H., Lee, S. H., \& Ha, S. K. (2017). Neuroprotective effect of 6paradol enriched ginger extract by fermentation using Schizosaccharomyces pombe. Journal of Functional Foods, 31, 304-310.

Ebrahimzadeh Attari, V., Malek Mahdavi, A., Javadivala, Z., Mahluji, S., Zununi Vahed, S., \& Ostadrahimi, A. (2018). A systematic review of the anti-obesity and weight lowering effect of ginger (Zingiber officinale Roscoe) and its mechanisms of action. Phytotherapy Research, 32(4), 577-585.

Fahmi, A., Hassanen, N., Abdur-Rahman, M., \& Shams-Eldin, E. (2019). Phytochemicals, antioxidant activity and hepatoprotective effect of ginger (Zingiber officinale) on diethylnitrosamine toxicity in rats. Biomarkers, 24(5), 436-447.

Ganjre, A., Kathariya, R., Bagul, N., \& Pawar, V. (2015). Anti-carcinogenic and anti-bacterial properties of selected spices: implications in oral health. Clinical Nutrition Research, 4(4), 209-215.

Ghasemzadeh, A., Jaafar, H. Z., \& Rahmat, A. (2010). Antioxidant activities, total phenolics and flavonoids content in two varieties of Malaysia young ginger (Zingiber officinale Roscoe). Molecules, 15(6), 4324-4333.

Ghayur, M. N., \& Gilani, A. H. (2005). Ginger lowers blood pressure through blockade of voltagedependent calcium channels. Journal of Cardiovascular Pharmacology, 45(1), 74-80.

Hajimoosayi, F., Sadatmahalleh, S. J., Kazemnejad, A., \& Pirjani, R. (2020). Effect of ginger on the blood glucose level of women with gestational diabetes mellitus (GDM) with impaired glucose tolerance test (GTT): a randomized double-blind placebo-controlled trial. BMC Complementary Medicine and Therapies, 20(1), 1-7.

Hamza, A. A., Heeba, G. H., Hamza, S., Abdalla, A., \& Amin, A. (2021). Standardized extract of ginger ameliorates liver cancer by reducing proliferation and inducing apoptosis through inhibition oxidative stress/inflammation pathway. Biomedicine \& Pharmacotherapy, 134, 111102.

Haridas, M., Sasidhar, V., Nath, P., Abhithaj, J., Sabu, A., \& Rammanohar, P. (2021). Compounds of Citrus medica and Zingiber officinale for COVID-19 inhibition: in silico evidence for cues from Ayurveda. Future Journal of Pharmaceutical Sciences, 7(1), 1-9.

Honarvar, N. M., Zarezadeh, M., Khorshidi, M., Arzati, M. M., Yekaninejad, M. S., Abdollahi, M., ... \& Saedisomeolia, A. (2019). The effect of an oral ginger supplementation on NF-kB concentration in peripheral blood mononuclear cells and anthropomorphic data of patients with type 2 diabetes: A randomized double-blind, placebo-controlled clinical trial. Complementary Therapies in Medicine, 42, 7-11.

Hussein, U. K., Hassan, N. E. H. Y., Elhalwagy, M. E., Zaki, A. R., Abubakr, H. O., Nagulapalli Venkata, K. 
C., ... \& Bishayee, A. (2017). Ginger and propolis exert neuroprotective effects against monosodium glutamate-induced neurotoxicity in rats. Molecules, 22(11), 1928.

Kaushik, S., Jangra, G., Kundu, V., Yadav, J. P., \& Kaushik, S. (2020). Anti-viral activity of Zingiber officinale (Ginger) ingredients against the Chikungunya virus. Virusdisease, 31(3), 270-276.

Khare, C. P. (2008). Indian Medicinal Plants: an Illustrated Dictionary. Springer Science \& Business Media.

Kravchenko, I., Eberle, L., Nesterkina, M., \& Kobernik, A. (2019). Anti-inflammatory and analgesic activity of ointment based on dense ginger extract (Zingiber officinale). Journal of Herbmed Pharmacology, 8(2), 126-132.

Kritikar, K. R., Basu, B. D., \& Blatter, E. (1993). Indian Medicinal Plants. Vol. 4. $2^{\text {nd }}$ edition. International Book Distributers, Dehradun, India.

Kulkarni, R. A., \& Deshpande, A. R. (2016). Anti-inflammatory and antioxidant effect of ginger in tuberculosis. Journal of Complementary and Integrative Medicine, 13(2), 201-206.

Lai, Y. S., Lee, W. C., Lin, Y. E., Ho, C. T., Lu, K. H., Lin, S. H., ... \& Sheen, L. Y. (2016). Ginger essential oil ameliorates hepatic injury and lipid accumulation in high fat diet-induced nonalcoholic fatty liver disease. Journal of Agricultural and Food Chemistry, 64(10), 2062-2071.

Li, C., Li, J., Jiang, F., Tzvetkov, N. T., Horbanczuk, J. O., Li, Y., ... \& Wang, D. (2021). Vasculoprotective effects of ginger (Zingiber officinale Roscoe) and underlying molecular mechanisms. Food \& Function, 12(5), 1897-1913.

Liu, Q., Liu, J., Guo, H., Sun, S., Wang, S., Zhang, Y., ... \& Qiao, Y. (2013). [6]-Gingerol: a novel AT1 antagonist for the treatment of cardiovascular disease. Planta Medica, 79(05), 322-326.

Lucky, E., Igbinosa, O. E., \& Jonahan, I. (2017). Antimicrobial activity of Zingiber officinale against multidrug resistant microbial isolates. Health Sciences Research, 4(6), 76-81.

Ma, S. Q., Guo, Z., Liu, F. Y., Hasan, S. G., Yang, D., Tang, N., .. \& Tang, Q. Z. (2021). 6-Gingerol protects against cardiac remodeling by inhibiting the p38 mitogen-activated protein kinase pathway. Acta Pharmacologica Sinica, 1-12.

Mohammed, W. F., Saleh, B. H., Ibrahim, R. N., \& Hassan, M. B. (2019). Antibacterial activity of Zingiber officinale (Ginger) against clinical bacterial isolates. South Asian Journal of Research in Microbiology, 1-7.

Mozaffari-Khosravi, H., Naderi, Z., Dehghan, A., Nadjarzadeh, A., \& Fallah Huseini, H. (2016). Effect of ginger supplementation on proinflammatory cytokines in older patients with osteoarthritis: outcomes of a randomized controlled clinical trial. Journal of Nutrition in Gerontology and Geriatrics, 35(3), 209218.

Mukherjee, P. K., Harwansh, R. K., Bahadur, S., Banerjee, S., Kar, A., Chanda, J., ... \& Katiyar, C. K. (2017). Development of Ayurveda-tradition to trend. Journal of Ethnopharmacology, 197, 10-24.

Nabil, L. M., El-Zainy, M. A., \& Hassan, R. (2021). Effect of Spirulina and Ginger Against Radiation Hazards on Mandibular Alveolar Bone of Albino Rats (Histological, Immunohistochemical and Radiographic Study). Egyptian Journal of Histology.

Nam, Y. H., Hong, B. N., Rodriguez, I., Park, M. S., Jeong, S. Y., Lee, Y. G., ... \& Kang, T. H. (2020). Steamed ginger may enhance insulin secretion through KATP channel closure in pancreatic $\beta$-cells potentially by increasing 1-dehydro-6-gingerdione content. Nutrients, 12(2), 324.

Nunes Carvalho, G. C., Garcia Lira Neto, J. C., Cunha Nunes, L. C., Parente Garcia Alencar, A. M., Lino Marques, R. L., \& Coelho Damasceno, M. M. (2021). Effectiveness of Ginger in the Treatment of Type 2 Diabetes Mellitus: APilot Study of the Randomized Clinical Trial Type. Aquichan, 21(1).

Ojulari, L. S., Olatubosun, O. T., Okesina, K. B., \& Owoyele, B. V. (2014). The effect of Zingiber officinale 
(ginger) extract on blood pressure and heart rate in healthy humans. IOSR J Dent Med Sci, 10, 76-8.

Oliveira, C. T., Ramos, A. L. C. C., Mendonça, H. D. O. P., Consenza, G. P., Silva, M. R., Fernandes, C., ... \& de Araújo, R. L. B. (2020). Quantification of 6-gingerol, metabolomic analysis by paper spray mass spectrometry and determination of antioxidant activity of ginger rhizomes (Zingiber officinale). Research, Society and Development, 9(8), e366984822-e366984822.

Pragasam, S. S. J., Birineni, S., Shyam, P., Nagasuryaprasad, K., \& Venkatesan, V. (2021). Therapeutic efficacy of 6-Gingerol and 6-Shogaol in promoting browning of white adipocytes vis-à-vis enhanced thermogenesis portrayed in high fat milieu. Food Bioscience, 101211.

Sampath, S. J. P., Birineni, S., Perugu, S., Kotikalapudi, N., \& Venkatesan, V. (2021). Therapeutic efficacy of 6-Gingerol and 6-Shogaol in promoting browning of white adipocytes vis-à-vis enhanced thermogenesis portrayed in high fat milieu. Food Bioscience, 42, 101211.

Ramudu, S. K., Korivi, M., Kesireddy, N., Chen, C. Y., Kuo, C. H., \& Kesireddy, S. R. (2011). Ginger feeding protects against renal oxidative damage caused by alcohol consumption in rats. Journal of Renal Nutrition, 21(3), 263-270.

Rodrigues, F. A., Prata, M. M., Oliveira, I. C., Alves, N. T., Freitas, R. E., Monteiro, H. S., ... \& Havt, A. (2014). Gingerol fraction from Zingiber officinale protects against gentamicin-induced nephrotoxicity. Antimicrobial Agents and Chemotherapy, 58(4), 1872-1878.

San Chang, J., Wang, K. C., Yeh, C. F., Shieh, D. E., \& Chiang, L. C. (2013). Fresh ginger (Zingiber officinale) has anti-viral activity against human respiratory syncytial virus in human respiratory tract cell lines. Journal of Ethnopharmacology, 145(1), 146-151.

Saneei Totmaj, A., Emamat, H., Jarrahi, F., \& Zarrati, M. (2019). The effect of ginger (Zingiber officinale) on chemotherapy-induced nausea and vomiting in breast cancer patients: A systematic literature review of randomized controlled trials. Phytotherapy Research, 33(8), 1957-1965.

Saputri, F. C., Nabila, N., \& Mun'im, A. (2017). Combination of Ginger and Sappan Wood Extract Effect on In vivo Antithrombotic Activity Test. Journal of Young Pharmacists, 9(1), S46.

Shahzad, F., Anderson, D., \& Najafzadeh, M. (2020). The antiviral, anti-inflammatory effects of natural medicinal herbs and mushrooms and SARS-CoV-2 infection. Nutrients, 12(9), 2573.

Sharma, B. K., Klinzing, D. C., \& Ramos, J. D. (2015). Modulatory activities of Zingiber officinale Roscoe methanol extract on the expression and activity of MMPs and TIMPs on dengue virus infected cells. Asian Pacific Journal of Tropical Disease, 5, S19-S26.

Sharma, P. (2019) Dravyagun Vigyan. Chaukambha Bharti Academy, Volume 2, Varanasi, India. ISBN: 9789384541040

PR, S. A., \& Prakash, J. (2010). Chemical composition and antioxidant properties of ginger root (Zingiber officinale). Journal of Medicinal Plants Research, 4(24), 2674-2679.

Silver, R. J. (2007). Ayurvedic veterinary medicine: principles and practices. Veterinary Herbal Medicine, 59-83. http://www.theplantlist.org

The Plant List (2013). Version 1. Published on the Internet; http://www.theplantlist.org

Thomson, M., Al-Qattan, K. K., Al-Sawan, S. M., Alnaqeeb, M. A., Khan, I., \& Ali, M. (2002). The use of ginger (Zingiber officinale Rosc.) as a potential anti-inflammatory and antithrombotic agent. Prostaglandins, Leukotrienes and Essential Fatty Acids, 67(6), 475-478.

Tramontin, N. D. S., Luciano, T. F., Marques, S. D. O., de Souza, C. T., \& Muller, A. P. (2020). Ginger and avocado as nutraceuticals for obesity and its comorbidities. Phytotherapy Research, 34(6), 12821290.

Venkateswaran, M., Jayabal, S., Hemaiswarya, S., Murugesan, S., Enkateswara, S., Doble, M., \& Periyasamy, S. (2021). Polyphenol-rich Indian ginger cultivars ameliorate GLUT4 activity in C2C12 
cells, inhibit diabetes-related enzymes and LPS-induced inflammation: An in vitro study. Journal of Food Biochemistry, 45(2), e13600.

Wang, J., Ke, W., Bao, R., Hu, X., \& Chen, F. (2017). Beneficial effects of ginger Zingiber officinale Roscoe on obesity and metabolic syndrome: a review. Annals of the New YorkAcademy of Sciences, 1398(1), 83-98.

Wang, J., Li, D., Wang, P., Hu, X., \& Chen, F. (2019). Ginger prevents obesity through regulation of energy metabolism and activation of browning in high-fat diet-induced obese mice. The Journal of Nutritional Biochemistry, 70, 105-115.

Wang, J., Prinz, R. A., Liu, X., \& Xu, X. (2020a). In vitro and in vivo antiviral activity of gingerenone A on influenza $A$ virus is mediated by targeting janus kinase 2. Viruses, 12(10), 1141.

Wang, X., Shen, Y., Thakur, K., Han, J., Zhang, J. G., Hu, F., \& Wei, Z. J. (2020b). Antibacterial activity and mechanism of ginger essential oil against Escherichia coli and Staphylococcus aureus. Molecules, 25(17), 3955.

World Health Organization. (2003). WHO Guidelines on Good Agricultural and Collection Practices (GACP) for Medicinal Plants. World Health Organization.

Yeh, H. Y., Chuang, C. H., Chen, H. C., Wan, C. J., Chen, T. L., \& Lin, L. Y. (2014). Bioactive components analysis of two various gingers (Zingiber officinale Roscoe) and antioxidant effect of ginger extracts. LWT-Food Science and Technology, 55(1), 329-334.

Zhang, M. M., Wang, D., Lu, F., Zhao, R., Ye, X., He, L., ... \& Wu, C. J. (2021). Identification of the active substances and mechanisms of ginger for the treatment of colon cancer based on network pharmacology and molecular docking. BioData Mining, 14(1), 1-16. 


\title{
Biotechnological Applications of Phototrophic Purple Nonsulfur Bacteria
}

\author{
Anannya Banerjee, Soumita Debnath, Trishita Mukherjee, Baishali Pandit* \\ Department of Botany, Surendranath College, Kolkata, India \\ ${ }^{*}$ Corresponding Author's Email: baishalipandit@gmail.com
}

\begin{abstract}
The physiological and biochemical characteristics of phototrophic purple nonsulfur bacteria (PPNSB) are unique. These microbes may grow and thrive in a variety of different environments. There are additional reports that emphasize their ecological importance. Phototrophic purple nonsulfur bacteria (PPNSB) are Gram-negative anoxyphototrophic bacteria that do not accumulate sulphur in their cells. They include members of the $\alpha$ and $\beta$-Proteobacteria families. PPNSB produces extracellular polymeric substances (EPS), which is one of several polymeric compounds released by this group of bacteria due to their metabolic versatility (various intracellular as well as extracellular substances are secreted by PPNSB). Carbohydrates, proteins, lipids, DNA, uronic acid, humic compounds, and other organic remnants constitute the chemical composition of extracellular polymeric substances (EPS). EPS is primarily produced by phototrophic purple nonsulfur bacteria (PPNSB) to aid in the development of microbial colonies or aggregates (or biofilms) at or outside the cell surface during their growth phase in suspended cultures. Individual bacteria may establish sustainable colonies and survive in stable circumstances at high cell densities in the presence of other mixed populations creating a community or colony, and they can also defend microbial biofilms from different environmental stressors. Different activities, including active secretion, shedding of cell surface material, cell lysis, and adsorption from the environment, all influence the composition of EPS. Phototrophic purple non-sulfur bacteria have recently been investigated for biotechnological applications, including wastewater treatment, aquaculture, bioremediation, biopolymer production, and biohydrogen production. These microorganisms' EPS has also been utilised in cancer therapy drug delivery systems (DDS).
\end{abstract}

\section{Keywords: PPNSB; EPS; Biofilms; Wastewater Treatment; Bioremediation}

\section{INTRODUCTION}

Phototrophic purple nonsulfur bacteria (PPNSB) are a diverse group of microbes found in a variety of environments (Caumette et al. 2004), including waste water ponds, sediments, moist soils, seawater pools, hypersaline environments (Okubo, Futamata \& Hiraishi, 2006), frozen lakes (Karr et al., 2003), sewage, waste lagoons (Kobayashi, 1975), and more. They occasionally create dense blooms in settings with low or imperceptible sulphide levels.

Phototrophic purple nonsulfur bacteria (PPNSB) are a biologically diverse community that thrives in both light and darkness. PPNSB can develop photoautotrophically under phototrophic (anoxic/light) conditions using hydrogen or limited amounts of sulphide as electron donors; a few species can employ sulfite or $\mathrm{Fe} 2+$ as photosynthetic electron donors. Most PPNSB, on the other hand, thrive as photoheterotrophs in conditions containing a readily utilizable organic source such as malate or pyruvate, as well as ammonium as a nitrogen source. The majority of PPNSB require yeast extract. Photoheterotrophic development of PPNSB is aided by organic acids, amino acids, fatty acids, alcohols, and carbohydrates. PPNSB are good nitrogen-fixing bacteria, with a few exceptions. Rhodobacter capsulatus and Rhodobacter sphaeroides grow the fastest when given just nitrogen and have the greatest levels of in vivo nitrogenase activity (Madigan, 1984).

The EPS generated by PPNSB is made up of a range of chemical compounds (Irie, Preston \& Yuk, 2006; 
Flemming, Neu \& Wozniak, 2007). Carbohydrate was discovered to be the most abundant element in the EPS of several pure cultures (Cescutti et al., 1999), but protein was also found in significant amounts on occasion (Veiga et al., 1997). Tsuneda et al., (2001) found humic material, uronic acid, and deoxyribonucleic acids (DNA) in EPS. Carbohydrates have been discovered as one of the primary components of EPS, and it is clear that these polymers are produced by a wide range of phototrophic bacteria, with an equally large diversity of chemical configurations (Wingender, Neu \& Flemming, 1999).

PPNSB have recently been reported as having numerous biotechnological applications (Schumacher \& Sekoulov, 2002, Bender \& Phillips, 2004, Watanabe et al., 2003, Panwichian et al., 2012, Liebergesell et al., 1991, Nath, Kumar \& Das, 2005, Takeno, Yamaoka \& Sasaki, 2005).

\section{LITERATURE REVIEW}

The generation and characterisation of EPS from different pure and mixed cultures, activated sludge, granular sludge, and biofilm in wastewater treatment systems has received a lot of attention. However, there is limited information on the features of PPNSB's Extracellular Polymeric Substances. Rhodovulum sp., a marine photosynthetic bacterium, was identified, and its extracellular nucleic acids in the EPS were discovered to be important for its flocculation (Watanabe et al., 1998). Different techniques were used to extract extracellular polymeric substances (EPS) from Rhodopseudomonas acidophila, with EDTA extraction being the most efficient. EPS from R. acidophila had carbohydrate, protein, and nucleic acid concentrations of $6.5,58.4$, and $5.4 \mathrm{mg} \mathrm{g-1}$ dry cells, respectively (Sheng, Yu \& Yu, 2005). It was also shown that addition of hazardous chemicals to the growing media such as $\mathrm{Cu}(\mathrm{II}), \mathrm{Cr}(\mathrm{VI}), \mathrm{Cd}(\mathrm{II})$, and 2,4-dichlorophenol (2,4-DCP) increased the synthesis of EPS by R. acidophila while lowering the cell dry weight. The EPS content rose by $5.5,2.5,4.0$, and 1.4 times, respectively, at doses of $30 \mathrm{mg} \mathrm{l-1}$ $\mathrm{Cu}(\mathrm{II}), 40 \mathrm{mg} \mathrm{l}-1 \mathrm{Cr}(\mathrm{VI}), 5 \mathrm{mg} \mathrm{l}-1 \mathrm{Cd}(\mathrm{II})$, and $100 \mathrm{mg} \mathrm{l}-1$ 2,4-DCP, compared to the control. This result implies the influence of xenobiotics on the synthesis of these extracellular polymeric substances. The proteins/carbohydrates ratio of EPS was also substantially affected by the presence of these hazardous compounds (Sheng, Yu \& Yu, 2005).

The disposal of hazardous wastes generated by industry frequently results in significant environmental pollution. Contaminants present in industrial wastewaters, such as structurally complex organic compounds, recalcitrant and xenobiotic molecules, and heavy metals, can create major environmental and human health concerns. PPNSB that are able to withstand the presence of toxicants and live in these polluted settings often devise certain mechanisms or processes by which they can survive in the stressful environment. These mechanisms may include amelioration or transformation of harmful chemical compounds into some other less toxic forms (Cokgor et al., 2004; Chen et al., 2008), these processes have been identified and reported to be very useful and significant ones, and they are highly effective even when the organic load is on the higher side (especially in tropical areas where sun exposure is maximum). Treatment of oil containing sewage wastewater by successful transformation of hydrocarbon (Takeno, Yamaoka \& Sasaki, 2005), resistance to various heavy metals (Panwichian et al., 2010), reduction of heavy metals to less harmful forms (Nepple, Kessi \& Bachofen, 2000) and their extensive evaluation for heavy metal bioremediation potential (Watanabe et al., 2003; Feng et al., 2007; Bai et al., 2008) are some of the attractive applications of photosynthetic bacteria. Furthermore, BTEX hydrocarbons, polycyclic aromatic hydrocarbons, microaromatics, polychlorinated biphenyls, chlorinated phenols, and aliphatics are also targets for PPNSB's bioremediation technology (Singh, Paul \& Jain, 2006; Lynch \& Moffat, 2005).

Extracellular polymeric substances (EPS) produced by Phototrophic purple nonsulfur bacteria (PPNSB) are recently been studied as agents for biosorption of heavy metals, because of their ability to bind heavy metals and grow profusely even at very high metal concentrations (Watanabe et al., 2003; lyer, Mody \& Jha, 2005). Natural detoxification of heavy metal-contaminated regions is carried out by metal chelating properties of EPS, this same principle applies to bioremediation as well (Flemming \& Wingender, 2001). Functional groups such as carboxyl, phosphate, sulphate, and their ligand binding preferences for certain metals mainly control the metal-binding capacity of the EPS matrix. The culinary, pharmaceutical, biomedical, bioremediation, and bioleaching sectors are all interested in EPS produced 
by PPNSB because of their wide structural variation and unique physical, rheological, and other characteristics (Fialho et al., 2008; Kumar \& Mody, 2009). Because of their ability to bind metal ions from solutions and their role in flocculation, EPS is critical in the removal of heavy metals from the environment (Pal \& Paul, 2008).

Table 1: Summarizing the Products of Biotechnological Importance Synthesized by Various Phototrophic Purple Nonsulfur Bacteria (PPNSB)

\begin{tabular}{|c|c|c|}
\hline Purple Non Sulfur Bacteria & Product & Uses \\
\hline $\begin{array}{c}\text { Rhodospirillum rubrum, } \\
\text { Rhodobacter sphaeroides } \\
\text { Rhodobacter capsulatus }\end{array}$ & $\begin{array}{c}\text { Polyhydroxyalkanoate } \\
\text { (PHA) production }\end{array}$ & $\begin{array}{c}\text { Biopolyesters (alternative to } \\
\text { conventional plastics as they are } \\
\text { biodegradable and biocompatible) }\end{array}$ \\
\hline Rhodovulum sulfidophilum & $\begin{array}{c}\text { Extracellular nucleic } \\
\text { acids (DNA and RNA) }\end{array}$ & $\begin{array}{c}\text { Biofilm formation and horizontal } \\
\text { gene transfer }\end{array}$ \\
\hline $\begin{array}{c}\text { Rhodovulum sulfidophilum } \\
\text { Rhodobacter sphaeroides } \\
\begin{array}{c}\text { Rhodobacter palustris } \\
\text { Rhodovulum sulfidophilum }\end{array}\end{array}$ & $\begin{array}{c}\text { Photohydrogen } \\
\text { production }\end{array}$ & $\begin{array}{c}\text { Aiological research and medicines } \\
\text { for RNA-based therapy }\end{array}$ \\
\hline $\begin{array}{c}\text { Rhodopseudomonas } \\
\text { acidophila Rhodovulum sp }\end{array}$ & $\begin{array}{c}\text { Extracellular polymeric } \\
\text { substances (EPS) } \\
\text { production }\end{array}$ & Heavy metal bioremediation \\
\hline
\end{tabular}

\section{DISCUSSION}

Sarkar and Banerjee, 1980; Sasikala and Ramana, 1995 and Archana et al., 2004 and several other workers have studied phototrophic purple nonsulfur bacteria (PPNSB) isolated from natural and artificial environments in India from a taxonomic and physiological standpoint, and their biotechnological potentials for the production of biohydrogen and biodegradable biopolymer, polyhydroxyalkanoates have also been evaluated (Sasikala and Ramana, 1995; Sunita \& Mitra, 1993; Archana et al., 2004; Basak \& Das, 2009; Mukhopadhyay, Patra \& Paul, 2005). Among other things, PPNSB may be utilized to improve plant growth performance, increase yield and quality of edible plant biomass, improve resilience to environmental stresses, bioremediate heavy metals, and reduce greenhouse gas emissions (Sakarika et al., 2020). Extracellular polymeric substances (EPS) produced by these microorganisms not only facilitate biofilm formation, thereby increasing their chances of survival but is also important regarding their bioremediation, bioleaching and biomedical applications.

\section{CONCLUSION}

The taxonomy, physiology, and biochemistry of phototrophic purple nonsulfur bacteria (PPNSB) from natural and man-made wastewater habitats in India have received very little attention. Furthermore, the synthesis of extracellular polymeric compounds by this diverse collection of microbes, as well as their potential for various biotechnological uses, are yet unexplored to a certain extent.

\section{ACKNOWLEDGEMENT}

The authors express their gratitude to the Department of Biotechnology, Government of India, for sponsoring this review study via the DBT-Star College Grant. We are also appreciative to Surendranath College's Principal and DBT-Star Coordinator for their assistance and encouragement in executing this review project at the undergraduate level. 


\section{REFERENCES}

Archana, A., Sasikala, C., Ramana, C. V., \& Arunasri, K. (2004). "Paraffin wax-overlay of pour plate", a method for the isolation and enumeration of purple non-sulfur bacteria. Journal of Microbiological Methods, 59(3), 423-425.

Bai, H. J., Zhang, Z. M., Yang, G. E., \& Li, B. Z. (2008). Bioremediation of cadmium by growing Rhodobacter sphaeroides: kinetic characteristic and mechanism studies. Bioresource Technology, 99(16), 7716-7722.

Basak, N., \& Das, D. (2009). Photofermentative hydrogen production using purple non-sulfur bacteria Rhodobacter sphaeroides OU 001 in an annular photobioreactor: a case study. Biomass and Bioenergy, 33(6-7), 911-919.

Bender, J., \& Phillips, P. (2004). Microbial mats for multiple applications in aquaculture and bioremediation. Bioresource Technology, 94(3), 229-238.

Caumette, P., Guyoneaud, R., Imhoff, J.F., Süling, J., and Gorlenko, V. (2004). Thiocapsa marina sp. nov., a novel, okenone-containing purple sulfur bacterium isolated from brackish coastal and marine environments. Int. J. Syst. Evol. Microbiol. 54, 1031-1036.

Cescutti, P., Toffanin, R., Pollesello, P., \& Sutherland, I. W. (1999). Structural determination of the acidic exopolysaccharide produced by a Pseudomonas sp. strain 1.15. Carbohydrate Research, 315(1-2), 159-168.

Chen, Z., Ren, N., Wang, A., Zhang, Z. P., \& Shi, Y. (2008). A novel application of TPAD-MBR system to the pilot treatment of chemical synthesis-based pharmaceutical wastewater. Water Research, 42(13), 3385-3392.

Cokgor, E. U., Alaton, I. A., Karahan, O., Dogruel, S., \& Orhon, D. (2004). Biological treatability of raw and ozonated penicillin formulation effluent. Journal of Hazardous Materials, 116(1-2), 159-166.

Feng, Y., Yu, Y., Wang, Y., \& Lin, X. (2007). Biosorption and bioreduction of trivalent aurum by photosynthetic bacteria Rhodobacter capsulatus. Current Microbiology, 55(5), 402-408.

Fialho, A. M., Moreira, L. M., Granja, A. T., Popescu, A. O., Hoffmann, K., \& Sá-Correia, I. (2008). Occurrence, production, and applications of gellan: current state and perspectives. Applied Microbiology and Biotechnology, 79(6), 889-900.

Flemming, H. C., \& Wingender, J. (2001). Relevance of microbial extracellular polymeric substances (EPSs)-Part I: Structural and ecological aspects. Water Science and Technology, 43(6), 1-8.

Flemming, H. C., Neu, T. R., \& Wozniak, D. J. (2007). The EPS matrix: the "house of biofilm cells". Journal of Bacteriology, 189(22), 7945-7947.

Irie, Y., Preston, A., \& Yuk, M. H. (2006). Expression of the primary carbohydrate component of the Bordetella bronchiseptica biofilm matrix is dependent on growth phase but independent of Bvg regulation. Journal of Bacteriology, 188(18), 6680-6687.

Iyer, A., Mody, K., \& Jha, B. (2005). Biosorption of heavy metals by a marine bacterium. Marine Pollution Bulletin, 50(3), 340-343.

Karr, E. A., Sattley, W. M., Jung, D. O., Madigan, M. T., \& Achenbach, L. A. (2003). Remarkable diversity of phototrophic purple bacteria in a permanently frozen Antarctic lake. Applied and Environmental Microbiology, 69(8), 4910-4914.

Kobayashi, M. (1975). Role of photosynthetic bacteria in foul water purification. Progress in Water Technology.7, 309-315.

Kumar, A. S., \& Mody, K. (2009). Microbial exopolysaccharides: variety and potential applications. Microbial Production of Biopolymers and Polymer Precursors: Applications and Perspectives, 229253. 
Liebergesell, M., Hustede, E., Timm, A., Steinbüchel, A., Fuller, R. C., Lenz, R. W., \& Schlegel, H. G. (1991). Formation of poly (3-hydroxyalkanoates) by phototrophic and chemolithotrophic bacteria. Archives of Microbiology, 155(5), 415-421.

Lynch, J. M., \& Moffat, A. J. (2005). Bioremediation-prospects for the future application of innovative applied biological research. Annals of Applied Biology, 146(2), 217-221.

Madigan, M. T. (1984). A novel photosynthetic purple bacterium isolated from a Yellowstone hot spring. Science, 225(4659), 313-315.

Mukhopadhyay, M., Patra, A., \& Paul, A. K. (2005). Production of poly (3-hydroxybutyrate) and poly (3hydroxybutyrate-co-3-hydroxyvalerate) by Rhodopseudomonas palustris SP5212. World Journal of Microbiology and Biotechnology, 21(5), 765-769.

Nath, K., Kumar, A., \& Das, D. (2005). Hydrogen production by Rhodobacter sphaeroides strain OU 001 using spent media of Enterobacter cloacae strain DM11. Applied Microbiology and Biotechnology, 68(4), 533-541.

Nepple, B. B., Kessi, J., \& Bachofen, R. (2000). Chromate reduction by Rhodobacter sphaeroides. Journal of Industrial Microbiology and Biotechnology, 25(4), 198-203.

Okubo, Y., Futamata, H., \& Hiraishi, A. (2006). Characterization of phototrophic purple nonsulfur bacteria forming colored microbial mats in a swine wastewater ditch. Applied and Environmental Microbiology, 72(9), 6225-6233.

Pal, A., \& Paul, A. K. (2008). Microbial extracellular polymeric substances: central elements in heavy metal bioremediation. Indian Journal of Microbiology, 48(1), 49.

Panwichian, S., Kantachote, D., Wittayaweerasak, B., \& Mallavarapu, M. (2010). Isolation of purple nonsulfur bacteria for the removal of heavy metals and sodium from contaminated shrimp ponds. Electronic Journal of Biotechnology, 13(4), 3-4.

Panwichian, S., Kantachote, D., Wittayaweerasak, B., \& Mallavarapu, M. (2012). The use of selected purple nonsulfur bacteria to remove heavy metals and salts from sediment and water collected from contaminated areas to decrease their phytotoxicity. African Journal of Biotechnology, 11(29).

Sakarika, M., Spanoghe, J., Sui, Y., Wambacq, E., Grunert, O., Haesaert, G., ... \& Vlaeminck, S. E. (2020). Purple non-sulphur bacteria and plant production: benefits for fertilization, stress resistance and the environment. Microbial Biotechnology, 13(5), 1336-1365.

Sarkar, P. K., \& Banerjee, A. K. (1980). Nicotinic acid as an essential growth factor of Rhodospirillum photometricum and a new Rhodospirillum isolate. Naturwissenschaften 67, 41-42. https://doi.org/10.1007/BF00424507

Sasikala, C. H., \& Ramana, C. V. (1995). Biotechnological potentials of anoxygenic phototrophic bacteria. II. Biopolyesters, biopesticide, biofuel, and biofertilizer. Advances in Applied Microbiology, 41, 227-278.

Schumacher, G., \& Sekoulov, I. (2002). Polishing of secondary effluent by an algal biofilm process. Water Science and Technology, 46(8), 83-90.

Sheng, G. P., Yu, H. Q., \& Yu, Z. (2005). Extraction of extracellular polymeric substances from the photosynthetic bacterium Rhodopseudomonas acidophila. Applied Microbiology and Biotechnology, 67(1), 125-130.

Singh, R., Paul, D., \& Jain, R. K. (2006). Biofilms: implications in bioremediation. Trends in Microbiology, 14(9), 389-397.

Sunita, M., \& Mitra, C. K. (1993). Photoproduction of hydrogen by photosynthetic bacteria from sewage and waste water. Journal of Biosciences, 18(1), 155-160.

Takeno, K., Yamaoka, Y., \& Sasaki, K. (2005). Treatment of oil-containing sewage wastewater using 
immobilized photosynthetic bacteria. World Journal of Microbiology and Biotechnology, 21(8), 13851391.

Tsuneda, S., Park, S., Hayashi, H., Jung, J., \& Hirata, A. (2001). Enhancement of nitrifying biofilm formation using selected EPS produced by heterotrophic bacteria. Water Science and Technology, 43(6), 197-204.

Veiga, M. C., Jain, M. K., Wu, W., Hollingsworth, R. I., \& Zeikus, J. G. (1997). Composition and role of extracellular polymers in methanogenic granules. Applied and Environmental Microbiology, 63(2), 403-407.

Watanabe, M., Kawahara, K., Sasaki, K., \& Noparatnaraporn, N. (2003). Biosorption of cadmium ions using a photosynthetic bacterium, Rhodobacter sphaeroides $S$ and a marine photosynthetic bacterium, Rhodovulum sp. and their biosorption kinetics. Journal of Bioscience and Bioengineering, 95(4), 374-378.

Watanabe, M., Sasaki, K., Nakashimada, Y., Kakizono, T., Noparatnaraporn, N., \& Nishio, N. (1998). Growth and flocculation of a marine photosynthetic bacterium Rhodovulum sp. Applied Microbiology and Biotechnology, 50(6), 682-691.

Wingender, J., Neu, T. R., \& Flemming, H. C. (1999). What are bacterial extracellular polymeric substances?. In Microbial Extracellular Polymeric Substances (pp. 1-19). Springer, Berlin, Heidelberg. 


\title{
A Short Review on Gastric Cancer
}

\author{
Sneha Dey, Biswanath Dari, Subhrajit Kundu, Lalita Das* \\ Department of Chemistry, Surendranath College, Kolkata, India
}

${ }^{*}$ Corresponding Author's Email: das.lalita1@gmail.com

\begin{abstract}
Gastrointestinal cancer is another name for stomach cancer. Stomach cancer develops when healthy cells in the stomach become aberrant and proliferate uncontrollably. A malignant tumour in the stomach is possible. A cancerous tumour is often malignant, which means it can develop and spread to other regions of the body. Stomach Cancer can start in any part of the stomach. The stomach being affected by cancer, it has the potential to spread to the adjacent lymph nodes and other body regions. Stomach cancer can damage organs such as the liver, bones, lungs, and women's ovaries.
\end{abstract}

\section{Keywords: Stomach Cancer; Biologic Targeted Therapy; Diffuse Subtype; Helicobacter Pylori; Intestinal Subtype}

\section{INTRODUCTION}

Most of the stomach cancers are a type commonly known as adenocarcinoma, i.e., the cancers commenced within the glandular tissue that strains the inner of the stomach (Meyerhardt, Tepper \& Venook, 2015). Lymphoma, gastric sarcoma, and neuroendocrine tumours are just a few of the malignant tumours that can develop in the stomach (Li, 2020).

- Statistical Factor: Stomach cancer often occurs in older people. More than 64 percent of people are diagnosed with cancer at an older age. In general, it has been discovered that the majority of cancer patients are under the age of 68 . The occurrence of stomach cancer differs greatly around the world (Yusefi et al., 2018). However, stomach cancer is becoming less frequent, it is still the most common cancer variety and one of the deadliest mortality around the world. Stomach cancer originates at a sluggish rate and spreads slowly, which is why a human can survive for more than 5 years after being identified with stomach cancer. However, in some special cases, the survival rate may be higher or lower. Nearly 32 percent of people with stomach cancer, can survive for 5 years, suggesting that the majority of patients with stomach cancer are detected after the disease has spread to other parts of the body. In general, if stomach cancer is recognized before it has spread to other regions of the body, then the survival rate is increased depending on the stage of the cancer. 5-year survival rate is 69 percent if cancer is detected and treated before it spreads to the stomach's side. The survival percentage drops dramatically if cancer has spread to other regions of the body. However, the 5 -year survival rate plummets to approximately 5 percent when cancer has grown to a distant area of the body (Rawla \& Barsouk, 2019).

- Symptoms and Signs: Like other diseases, stomach cancer also has some symptoms

and signs, but they are very mild and very hard to notice by many people without a serious check-up. Due to the lack of particular symptoms, stomach cancer is commonly neglected at an early stage, people believe (Mallath, 2021; Mustafa et al., 2017).

The symptoms are:

o Indigestion or heart burn

o Uncomfortable sensations or pain in the abdomen

o Gastrointestinal discomfort after taking foods 
- Loss of appetite

- Bloating of the stomach after eating

o Sensation of food getting caught inside the throat whilst eating.

Besides, there could be some other signs also for stomach cancer, they are sickness and tiredness, blood in the stool and also while vomiting, and loss of weight which is unexplained.

\section{Types of treatment}

Stomach cancer is treated with a mix of therapies, just like any other cancer that doesn't have any specific treatment. In most of the cases stomach cancer can't be fully cured; the main reason behind this is often stomach cancer not found in an early stage until it goes to an advanced stage (Joshi \& Badgwell, 2021; Rausei \& Lianos, 2020).

There are several treatments available to treat stomach cancer, they are,

1. Surgery: Doing surgery, the tumour and some tissues from the affected part of the

stomach is removed during an operation. Different types of surgery are there to treat carcinoma, depending on the cancer's stage (Solsky \& In, 2021). Surgery is performed to remove the damaged portion of the stomach in a subtotal or partial gastrectomy, this process of surgery is called in the early stage of cancer when the cancer is cancer (Smyth et al., 2016). If the cancer has migrated to the outer part of the stomach, then not only just surgery is necessary. Besides this, chemotherapy or radiation treatment and chemotherapy is accustomed to treat the affected part of the stomach as well as the outer part of the stomach. Most importantly, when cancer is identified as stage 4 cancer, surgery is not recommended as a treatment.

\section{Radiation therapy: In this therapy, high energy X-rays are used to treat cancer}

affected cells. Radiation therapy is generally given over a specified length of time and consists of a certain number of sessions. People affected by gastric carcinoma, are generally given radiation therapy using an external beam by machine from outside of the human body. Radiation is used to reduce the size of the tumour before surgery and as well as to destroy any left cancer cell (Sitarz et al., 2018). Though this process of treatment is effective, but it has some side effects such as fatigue, mild skin reaction, upset stomach etc (Gao, 2016).

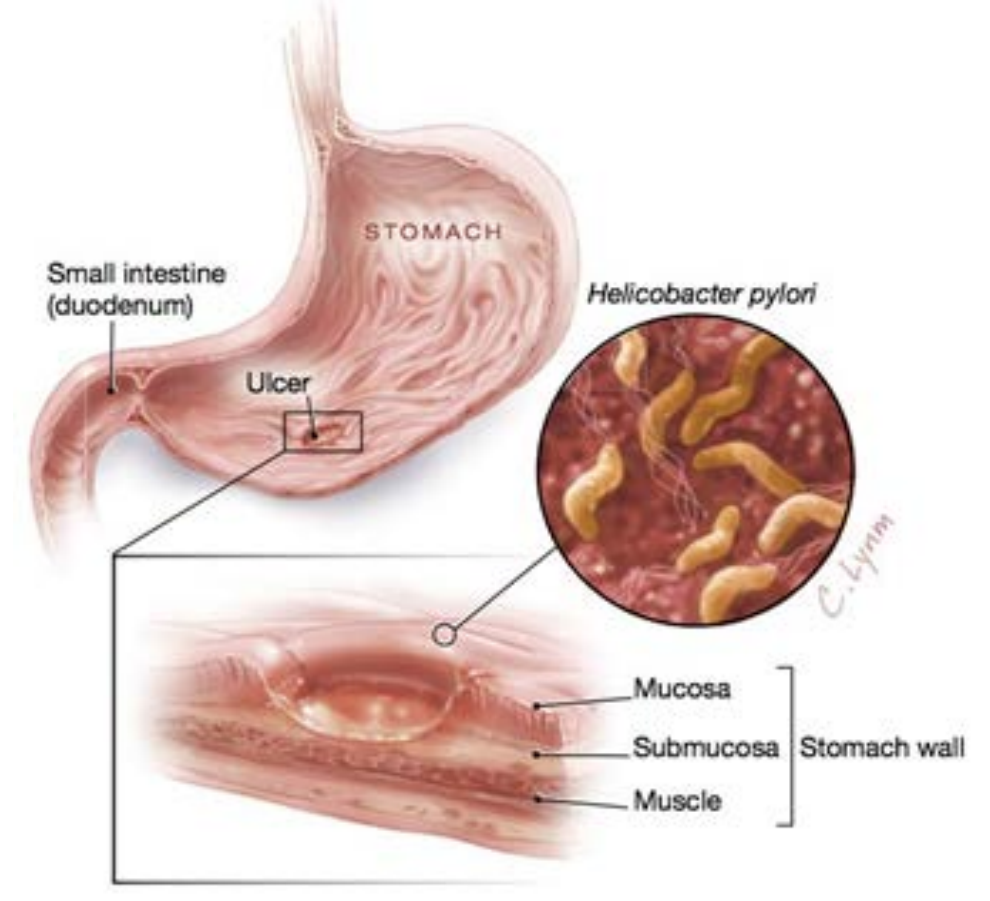

Figure 1: Gastric Carcinoma (Slomski, 2019) 
3. Chemotherapy: In treatment process drugs are used to stop rapidly spreading cancer

cells. The particular medicines for this treatment are called cytotoxic medicines (Beypınar \& Sümbül, 2020; Woell et al., 2008). It is generally a primary treatment of spreading cancer affected cells. Medicine attacks the cancer affected cells to kill those cells inside and as well as outside of stomach (Schipper \& Wagener, 1996).

4. Targeted therapy: Targeted therapies are used to identify and attack those proteins

that cancer calls have produced (Jomrich \& Schoppmann, 2016; Kharkar, 2020; Thiel \& Ristimäki, 2015). Two targeted medications for people suffering with stomach cancer are,

- Trastuzumab (Herceptin) (Guo et al., 2018): It mainly targets HER2, a protein that promotes cell growth. Sometimes cancer affected cells of stomach produces excess of HER2 (Demetriades, 2011; Won \& Janjigian, 2015; Zhu et al., 2021).

- Ramucirumab (CYRAMZA): This medication blocks a protein, named VEGF that encourages the body to produce the new blood vessels and hence tumour grows faster (Casak et al., 2015; Guglielmo et al., 2017).

5. Immunotherapy: By this process, medicine encourages the body's immune cells to

attack cancer affected cells (Matsueda \& Graham, 2014; Zhang et al., 2021a). This therapy is used when a candidate has received two or more other treatments (Dolcetti \& De, 2019).

\section{LITERATURE REVIEW}

\section{Pathologic subtypes and characteristics}

There are many classification schemes for gastric cancer, but the Lauren classification is the most commonly used one. The Lauren classification defines two main histological subtypes (Cisło et al., 2018; Hatogai, 2016).

\section{Classification of gastric cancer}

Each subtype represents distinct clinical and epidemiological characteristics. Gastric carcinomas with characteristics of both histologic subtypes are uncommon.

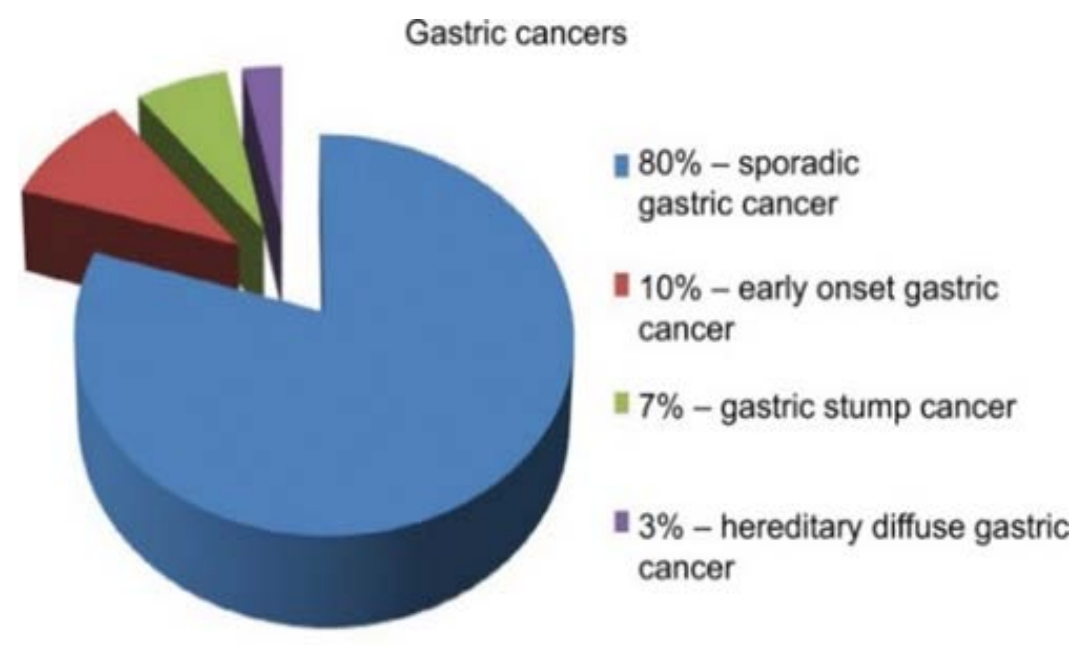

Figure 2: Classification of Gastric Cancer (Sitarz, 2018)

Molecules of intercellular adhesion, which are retained in intestinal form disease but deficient in diffuse stomach cancer, are responsible for the morphologic variations between the two subtypes. 


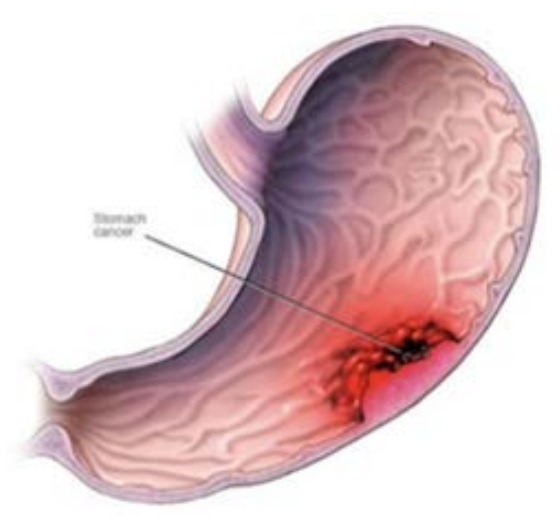

Figure 3: Intestinal Disease

Human epidermal growth factor receptor 2 (HER2) expression, for example, can be targeted in both subsets. In gastric cancer, amplification of gene is a common mode of gene change. Overexpression of such protein can be identified by IHC (Immuno-Histo Chemistry) (Chen et al., 2014). Colorectal, lung, stomach, and ovarian tumours have all been found to have HER2 amplification. In gastric carcinoma, HER2 (Duduyemi, Afihene \& Asiamah, 2017) over-expression has been reported in 7 to 34 percent of tumours, especially at the G-E junction and in lesions of the intestine. Overexpression of the Epidermal Growth Factor Receptor (EGFR) gene has been shown to be a marker for a poor prognosis in gastric cancer patients. In around 40 percent of stomach cancer patients, both Vascular Endothelial Growth Factor A(VEGFA) and its receptor are expressed, and higher VEGFA expression is linked to a poor prognosis and advanced illness. All of the above proteins have been investigated for their influence on survival and are potential targets for biologic treatment approaches. The clinical trials will be addressed in greater depth later in this study. New biomarkers (Chong et al., 2010) for targeted treatment, such as Fibroblast Growth Factor Receptor (FGFR) (Inokuchi et al., 2015), hepatocyte growth factor receptor and the mammalian target of rapamycin are being explored. Clinical trials are underway for some of the new targets (Mezhir et al., 2010).

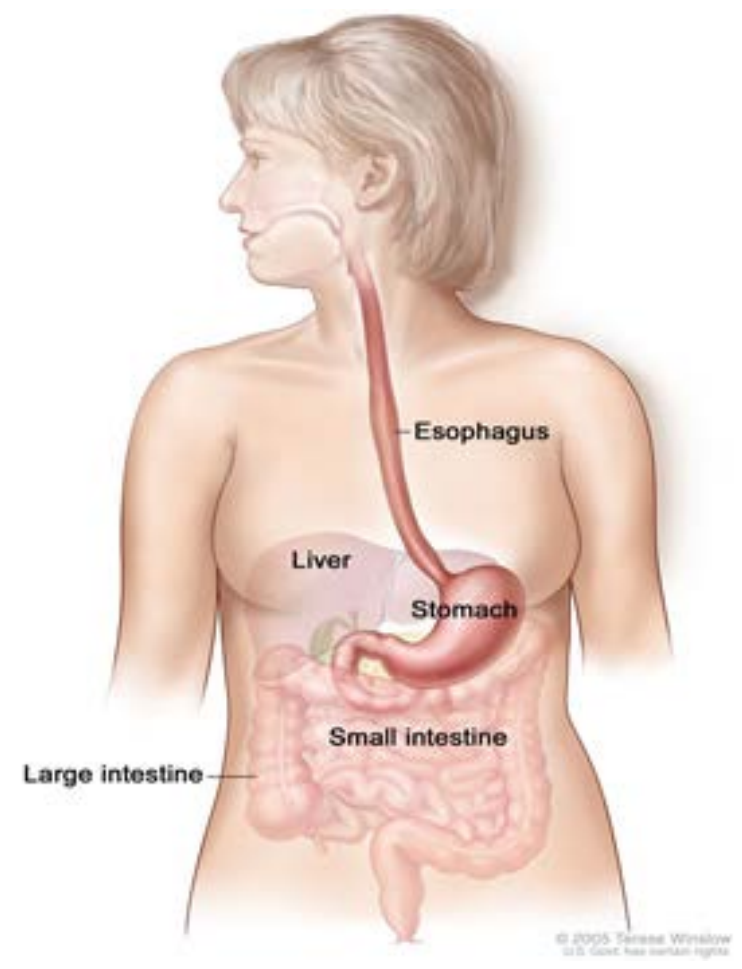

Figure 4: The Upper Gastrointestinal [Digestive] System Includes the Oesophagus and Stomach 


\section{Intestinal subtype}

In high-risk populations, the intestinal subtype is the most often histology that has been diagnosed, and it is more probable to be spontaneous than transmitted. Males are more likely than females to be diagnosed with it, and the tumours are likely to resemble ulcerated masses in appearance. $\mathrm{H}$. pylori infection is closely linked to the development of tumours. As a result, the intestinal subtype has been related to the global decrease in stomach cancer incidence throughout the previous several decades (Hu et al., 2012).

The $H$. pylori bacterium causes a sequence of alterations in histology that eventually lead to a cancerous lesion. The sequence of events starts with a long-term $\mathrm{H}$. pylori infection, which causes non-atrophic gastritis, which progresses to multifocal atrophic gastritis without metaplasia, followed by intestinal metaplasia and dysplasia (Akiyama \& Uemura, 2009). Long-term stomach inflammation caused by chronic $\mathrm{H}$. pylori infection can induce epithelial damage, leading in gastric atrophy marked by the loss of parietal and chief cells, as well as glandular atrophy (El-Zimaity et al., 2002). Intestinal metaplasia replaces the gastric epithelium, especially in the lesser curvature of the stomach, but it can occur elsewhere in the stomach. Dysplastic foci can form in regions of metaplasia over time. The lamina propria can be invaded by dysplastic areas over time, causing them to be classified as invasive malignancy by Western standards. In Japan ( Coronel-Castillo, Contreras-Carmona \& MéndezSánchez, 2018), carcinoma is described as extreme nuclear and architectural abnormalities without invasion.

Many oncogenes are over-expressed in intestinal subtype tumours; none, however, have been consistently present at any stage of the illness. The c-met oncogene is linked to the formation of around 20 percent of intestinal gastric cancers, and c-met transcript expression is linked to more advanced illness. In fact, certain virulent H. pylori ( Amieva \& Peek Jr, 2016) strains produce an effector protein that appears to alter c-met receptor signal transduction pathways, potentially leading to tumour initiation and development. Intestinal metaplasia, dysplasia, and invasive carcinomas have all been linked to K-ras mutations (Allgayer et al., 1999).

Many types of gastric malignancies in the intestine have mutations in tumour suppressor genes. The P53 tumour suppressor gene (TP53) (Saito et al., 2018) is an essential cell cycle regulator. In more than 60 percent of invasive gastric tumours, loss of heterozygosity or mutational inactivation of TP53 expression occurs. In H. pylori-associated chronic gastritis (Shtygasheva et al., 2010; Zhang et al., 2021b), metaplasia, and dysplasia, TP53 expression anomalies have also been observed; however, the link between the P53 tumour suppressor gene and $\mathrm{H}$. pylori is unclear. In addition to $\mathrm{H}$. pylori and gastric tumour initiation and proliferation, mutations in the adenomatous polyposis coli gene have been found in intestinal and diffuse subtype gastric tumours. Hypermethylation (Kim et al., 2004; Stanland \& Luftig, 2020) and micro-satellites can also be observed.

\section{Diffuse subtype}

Gastric cancer in its diffuse form tends to be more aggressive than gastric cancer in its intestinal form. It is more commonly diagnosed in younger individuals, and there is no gender bias (Ling et al., 2020). While it has been connected to $\mathrm{H}$. pylori infection, it is more commonly linked to loss of E-cadherin expression, and no precancerous lesions have been identified. Without gland development, diffuse gastric tumours tend to infiltrate the stomach wall and neighbouring organs, such as the duodenum and oesophagus. In certain cases, linitis plastica is caused by diffuse infiltration of the stomach wall. Signet ring cell histology ( serkan SENGER et al., 2020) is rarely seen in this subtype (Postlewait et al., 2015).

Diffuse gastric tumours have a definite carcinogenic mechanism involving faulty intercellular adhesions. The majority of instances are caused by a lack of expression of the cell adhesion protein E-cadherin (Liu \& Chu, 2014). The E-cadherin gene (CDH1) (Guilford et al., 1998) is a homo-dimeric transmembrane cellular adhesion protein involved in cell assembly: Complex of cell adhesion. The 
gene looks to be working as a tumour suppressor gene requiring a "Two-hit" model (Kosmider \& Moreau-Gachelin, 2006; Pizzo et al., 2021) if inactivation. HDGC is a hereditary type of diffuse gastric cancer with an autosomal dominant inheritance pattern. By the age of 80 , the disease has manifested itself early in life, with a lifetime cumulative chance of getting stomach cancer of more than 80 percent. Multifocal tumours under an intact mucosal surface are common in HDGC patients, making it harder to diagnose. Prophylactic gastrectomy is recommended for patients with $\mathrm{CDH} 1 \mathrm{germ}$-line mutations and a family history of gastric cancer (Mathers \& Loncar, 2006; Oliveira, Seruca \& Carneiro, 2006). $\mathrm{CDH} 1$ mutations have also been associated to sporadic diffuse gastric carcinomas. In sporadic diffuse type gastric tumours, mutations and loss of heterozygosity in the CDH1 gene, as well as promoter hypermethylation, are found (Wang et al., 2021).

\section{Helicobacter pylori}

One of the few cancers that can be linked to an infectious agent is gastric cancer. $\mathrm{H}$. pylori (Helicobacter pylori) (Abnet et al., 2011; Polk \& Peek, 2010; Suzuki \& Matsuzaki, 2018) is a spiral Gram-negative bacterium (Yang et al., 2021) that colonises roughly half of the world's population's stomachs. Chronic gastritis, peptic ulcer disease, and stomach lymphomas and non-cardiac adenocarcinomas (Huang et al., 2018) have all been related to it and is designated as a human carcinogen by the World Health Organization (Sitarz et al., 2018). While H. pylori infection is the strongest recognised risk factor for stomach cancer, only a tiny fraction of persons who are infected, develop cancer. Although both histologies may be causative, intestinal-type adenocarcinoma is more closely connected with $\mathrm{H}$. pylori (Ito et al., 2009) infections than the diffuse subtype of gastric cancer. The bacteria are considered to be responsible for 80 percent of instances of distal gastric cancer, although it has nothing to do with cardia gastric carcinoma. If left untreated, the infection is most often acquired during childhood and will last for the rest of one's life. A chronic infection's inflammation has the ability to generate reactive oxygen species, which can damage DNA. $\mathrm{H}$. pylori can also cause hypermethylation, particularly in CpG islands, which makes tumour suppressor genes (Tamura, 2006) inactive. The carcinogenicity of different bacteria strains varies. The cytotoxic-associated gene A (Cag A) (Parsonnet et al., 1997) is found in the more aggressive strains, and it codes for an oncogenic protein that may be delivered directly into stomach epithelial cells via type-IV secretion, triggering a sequence of molecular processes that leads to carcinogenesis. Cag A appears to be involved in the breakdown of intracellular junctions, epithelial polarity loss, increased proliferation, and reduced apoptosis, all of which lead to cancer development. The bulk of Cag A positive strains are found in high-risk locations like East Asia and the Colombian Andes, which helps to explain why the cancer is more frequent there. Another virulence factor that induces intracellular vacuoles is the vac $A$ protein (Crafciuc et al., 2021) and epithelial cells' membrane channels.

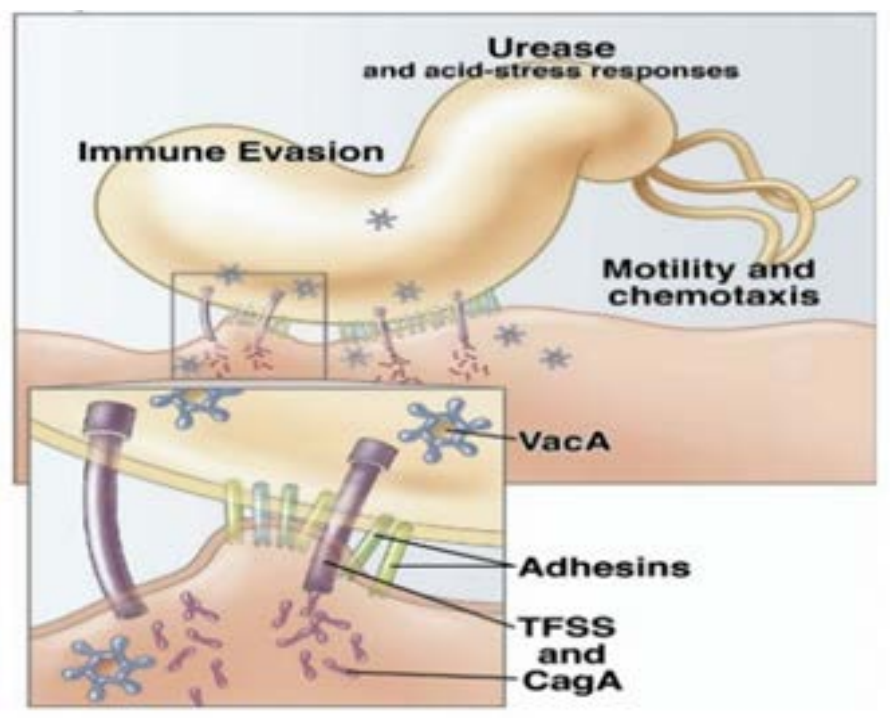

Figure 5: Helicobacter pylori Major Virulence and Colonization Factors (Amieva \& El-Omar, 2008) 
H. pylori eradication trials are logistically challenging because the infection causes carcinogenesis for several years. The current trials looked at how infection eradication affected the development and regression of precancerous lesions. After 15 years of follow-up, one study found a substantial decrease in the occurrence of stomach cancer in the population undergoing the therapy of $\mathrm{H}$. pylori (Dang \& Graham, 2017). According to a different study of the eradication trials, in the development of $\mathrm{H}$. pylori-associated stomach cancer, there is a limit beyond which treatment fails to reduce the risk of malignancy. The actual impact of the bacteria's elimination has yet to be determined.

\section{DISCUSSION}

\section{Global impact}

Gastric cancer has become one of the world's most common and deadly cancers. According to GLOBOCAN 2018 (Dar \& Sharma, 2019; Sharma, 2021) data gastric cancer is the 5th most frequent cancer and the 3rd most lethal around the globe. In the year of 2018 near about 783000 deaths were recorded. The incidence and mortality of gastric carcinoma varies greatly by area and are strongly influenced by diet and Helicobacter pylori infection. Gastric carcinoma is the third most common type of cancer in the world, behind only lung and colorectal cancer in total mortality, according to GLOBOCAN data from 2018. Gastric carcinoma is responsible for around 1 in every 12 oncological deaths. Every year, more than a million new instances of stomach cancer are discovered around the world. Gastric cancer is the 5th most often diagnosed cancer in the globe, and the 7 th most prevalent. From birth to age 74 , males have a 1.87 percent chance of developing gastric cancer, while females have a 0.79 percent chance. Up until the mid-1990s, the main cause of cancer-related mortality globally was gastric cancer. Gastric cancer kills nearly 783,000 men per year, putting it together the third most lethal carcinoma in men worldwide. Stomach cancer is the most common cause of death for men in ten countries around the world (Rampazzo et al., 2012) especially in eastern and central Asian countries including Iran, Kyrgyzstan, and Turkmenistan.

Table 1: Classification System of Gastric Carcinoma (Hu et al., 2012)

\begin{tabular}{|c|c|}
\hline Classification- World Health Organisation (2010) & Classification- Lauren (1965) \\
\hline $\begin{array}{l}\text { Adenocarcinoma in Papillary cells } \\
\text { Tubule shaped adenocarcinoma } \\
\text { Adenocarcinoma in Colorectal }\end{array}$ & Intestinal type \\
\hline $\begin{array}{l}\text { Carcinoma of the signet ring } \\
\text { And additional carcinomas with a low cohesive ability }\end{array}$ & Diffuse type \\
\hline Carcinoma multiforme & Indeterminate type \\
\hline $\begin{array}{l}\text { Carcinoma that affects gland-like cells in skin } \\
\text { Carcinoma that affects squamous cell in skin } \\
\text { Adenocarcinoma in liver } \\
\text { Lymphocytic stoma in carcinoma } \\
\text { Carcinoma in in a woman's uterus (womb) } \\
\text { Mixture of Carcinoma and Sarcoma } \\
\text { Carcinoma in Parietal cell } \\
\text { Tumours (for child) in soft tissues, kidneys, brain } \\
\text { Carcinoma in Parotid glands } \\
\text { Carcinoma in Paneth cells } \\
\text { Carcinoma in Primitive cells } \\
\text { Carcinoma in Gl and Pancreatobiliary tract } \\
\text { Tumour in the ovary or testicle } \\
\text { Carcinoma in the testicles } \\
\text { Tumour in the gonads } \\
\text { Adenocarcinoma in the thyroid (most often), breast, } \\
\text { pancreas, salivary glands (rare) }\end{array}$ & \\
\hline
\end{tabular}




\section{Therapeutic options}

Antibiotics are used to treat patients who have been infected with $\mathrm{H}$. pylori, is being studied to see whether or not it will help to avoid stomach cancer. Treatment of this infection has been shown in some studies to prevent pre-cancerous stomach anomalies, but further research is required. Antibiotics may aid in the prevention of stomach cancer, returning in some instances, but they are not true chemoprevention. Antibiotics have been proven to reduce the chance of cancer returning in another area of the stomach in patients who have had early-stage stomach cancer treated by endoscopic mucosal resection. Woefully, stomach cancers are more commonly discovered later in the United States, as a result, it's uncertain how beneficial these findings would be. People who take nonsteroidal anti-inflammatory medicines (NSAIDs) (Ong et al., 2007; Russell, 2001) are medications that are used to treat inflammation, NSAIDs like aspirin or ibuprofen may be less likely to get stomach cancer, according to some (but not all) research. Meanwhile, physicians usually advise against taking these medications only to reduce the cancer risk since they may have significant side effects in certain people. Chemo drugs function against cancer cells because they kill cells that divide quickly. Cancer cells, on the other hand, differ from normal cells in a number of ways. To try to exploit these gaps, researchers have created new targeted drugs in recent years. When traditional chemo drugs fail, targeted drugs can help. They also have fewer side effects than chemotherapy medications.

More research is needed to assess the effectiveness of eradicating $\mathrm{H}$. pylori as a stomach cancer preventive approach. A significant eradication rate has been observed when a proton pump inhibitor (PPI) (Coronel-Castillo, Contreras-Carmona \& Méndez-Sánchez, 2018) is used with two of the following three antibiotics: amoxicillin, clarithromycin, and nitroimidazole.<smiles>C[C@H]1N2C(=O)[C@@H](NC(=O)C(N)c3ccc(O)cc3)[C@H]2SC1(C)C</smiles>

Figure 6: Amoxicilin<smiles>O=[N+]([O-])c1cnc[nH]1</smiles>

Figure 7: Nitroimidazole

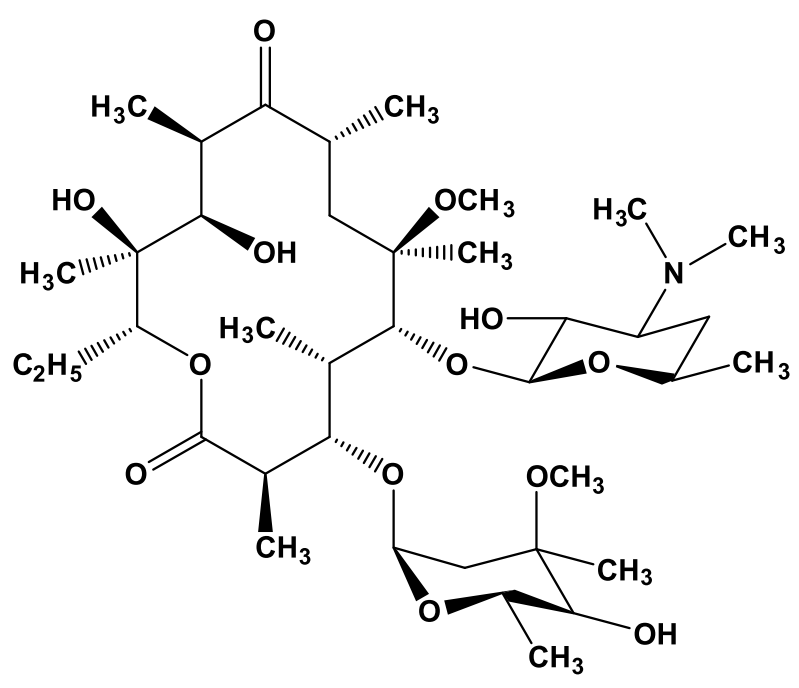

Figure 8: Clarithromycin 


\section{CONCLUSION}

Many university hospitals, medical facilities, and other institutions across the world are conducting important stomach cancer research. Scientists are gaining a better understanding of what causes the disease and how to cure it. Chemoprevention is the use of natural or manmade synthetic substances to lower cancer risks. In the prevention of stomach cancer, any substance can be beneficial. The process that causes gastric cancer are better understood than those that cause most of the other solid tumours. H. pylori is the single most important environmental risk factor for non-cardiovascular gastric cancer and at least some cardiac malignancies. Only by preventing the addition of $\mathrm{H}$ pylori or eradicating the infection before a yet-to-be-determined stage which is critical committal in gastric carcinogenesis, can gastric cancer be prevented. The data linking dietary variables to stomach cancer risk and the possibility for dietary change is inconsistent, and further study is needed. Gastric cancer mortality is reduced, and endoscopic surgery is made easier in high-risk populations, minimising the morbidity associated with the disease. Within, non-high-risk populations, it may be feasible to tailor screening to people who are most at risk of acquiring stomach cancer in the future. Age, family background, and $\mathrm{H}$. pylori status, as well as gastric atrophy is diagnosed by serological testing and probably in risk assessment, molecular genetic screening for predisposing polymorphisms and bacterial virulence factors is likely to be utilised.

Antioxidants are substances that combat free radicals. A free radical is a kind of molecule that is produced by several carcinogenic (cancer-causing) causes. Vital cell components such as chromosomes can be damaged by free radicals. The cells can die or become cancerous depending on the severity of the damage. Antioxidants are nutrients and other substances that may either kill or prevent free radical production. Vitamin C, beta-carotene, vitamin E, and the mineral selenium are among these nutrients. Studies on the use of dietary supplements to lower the incidence of stomach cancer have shown conflicting findings thus far. According to some evidence, using a combination of antioxidant supplements can lower the risk of stomach cancer in those who already have poor nutrition. In this field, further research is required.

\section{ACKNOWLEDGMENT}

The authors are thankful to Department of Chemistry, Surendranath College for providing us the latest information on this area.

\section{REFERENCES}

Abnet, C. C., Zheng, W., Ye, W., Kamangar, F., Ji, B. T., Persson, C., ... \& Chow, W. H. (2011). Plasma pepsinogens, antibodies against Helicobacter pylori, and risk of gastric cancer in the Shanghai Women's Health Study Cohort. British Journal of Cancer, 104(9), 1511-1516.

Akiyama, J., \& Uemura, N. (2009). Intestinal metaplasia subtype and gastric cancer risk. Journal of gastroenterology and hepatology, 24, 4-6. doi:10.1111/j.1440-1746.2008.05736.x

Allgayer, H., Wang, H., Shirasawa, S., Sasazuki, T., \& Boyd, D. (1999). Targeted disruption of the K-ras oncogene in an invasive colon cancer cell line down-regulates urokinase receptor expression and plasminogen-dependent proteolysis. British Journal of Cancer, 80(12), 1884-1891. doi:10.1038/ sj.bjc.6690616

Amieva, M. R., \& El-Omar, E. M. (2008). Host-bacterial interactions in Helicobacter pylori infection. Gastroenterology, 134(1), 306-323.

Amieva, M., \& Peek Jr, R. M. (2016). Pathobiology of Helicobacter pylori-induced gastric cancer. Gastroenterology, 150(1), 64-78. doi: 10.1053/j.gastro.2015.09.004

Beypınar, İ., \& Sümbül, A.T. (2020). Adjuvant chemotherapy in gastric cancer. Journal of Cancer Prevention \& Current Research, 11. doi:10.15406/jcpcr.2020.11.00431 
Casak, S. J., Fashoyin-Aje, I., Lemery, S. J., Zhang, L., Jin, R., Li, H., .. \& Pazdur, R. (2015). FDA approval summary: ramucirumab for gastric cancer. Clinical Cancer Research, 21(15), 33723376.doi:10.1158/1078-0432.CCR-15-0600

Chen, M., Li, Y., Ming, Z., Biao, A., \& Zheng, L. X. (2014). Comparison of HER2 status by fluorescence in situ hybridisation and immunohistochemistry in gastric cancer. Contemporary Oncology, 18(2), 95. doi:10.5114/wo.2014.41383

Chong, P. K., Lee, H., Zhou, J., Liu, S. C., Loh, M. C. S., Wang, T. T., ... \& Lim, Y. P. (2010). ITIH3 is a potential biomarker for early detection of gastric cancer. Journal of Proteome Research, 9(7), 36713679. doi:10.1021/pr100192h

Cisło, M., Filip, A. A., Offerhaus, G. J. A., Ciseł, B., Rawicz-Pruszyński, K., Skierucha, M., \& Polkowski, W. P. (2018). Distinct molecular subtypes of gastric cancer: from Laurén to molecular pathology. Oncotarget, 9(27), 19427. doi:10.18632/oncotarget.24827

Coronel-Castillo, C. E., Contreras-Carmona, J., \& Méndez-Sánchez, N. (2018). The proton pump inhibitors use and gastric cancer development: is it a real association. AMJ.

Crafciuc, A. V., Muñoz-Groza, A. E., Băleanu, V. D., Gheorman, V., Gheorman, V., Scurtu, I., ... \& Pîrvu, D. C. (2021). CLINICOPATHOLOGICAL ANALYSIS OF GASTRIC CANCER. Research and Science Today, (1), 39A-52.

Dang, B. N., \& Graham, D. Y. (2017). It is time to rethink H. pylori therapy. Journal of Gastrointestinal and Liver Diseases: JGLD, 26(2), 115.doi:10.15403/jgld.2014.1121.262.hpy

Dar, M., \& Sharma, K. (2019). Burden of cancer in India: GLOBOCAN 2018 estimates incidence, mortality, prevalence and future projections of cancer in India. J Emerg Technol Innovat Res, 6(6), e505. doi:10.1729/Journal.22750

Demetriades, H. (2011). HER2-Positive Gastric Cancer. Annals of Surgical Oncology, 18, 190. doi:10.1245/s10434-011-2000-0

Dolcetti, R., \& De Re, V. (2019). Immunomodulation and Immunotherapy for Gastric Cancer. Gastric Cancer In The Precision Medicine Era, 189-212.

Duduyemi, B. M., Afihene, M. Y., \& Asiamah, E. A. (2017). Human epidermal growth receptor-2 immunohistochemistry in gastric cancer. Cancer Research, 77, 753-753. doi:10.1158/15387445.AM2017-753

El-Zimaity, H. M., Ota, H., Graham, D. Y., Akamatsu, T., \& Katsuyama, T. (2002). Patterns of gastric atrophy in intestinal type gastric carcinoma. Cancer, 94(5), 1428-1436.doi:https://doi.org/ 10.1002/cncr.10375

Gao, L. (2016). The book Gastric Cancer: diagnosis and treatment of gastric cancer. Translational Gastroenterology and Hepatology, 1, 54-54. doi:10.21037/tgh.2016.06.04

Guglielmo, A., Ciani, O., Staropoli, N., Ciliberto, D., Tarricone, R., Giancotti, M., \& Mauro, M. (2017). OP60 Ramucirumab In Gastric Cancer Treatment: An Economic Evaluation. International Journal of Technology Assessment in Health Care, 33(S1), 26-27. doi:10.1017/S0266462317001453

Guilford, P., Hopkins, J., Harraway, J., McLeod, M., McLeod, N., Harawira, P., ... \& Reeve, A. E. (1998). E-cadherin germline mutations in familial gastric cancer. Nature, 392(6674), 402-405. doi:10.1038/32918

Guo, X., Zhu, H., Zhou, N., Chen, Z., Liu, T., Liu, F., ... \& Yang, Z. (2018). Noninvasive detection of HER2 expression in gastric cancer by 64Cu-NOTA-trastuzumab in PDX mouse model and in patients. Molecular Pharmaceutics, 15(11), 5174-5182. doi:10.1021/acs.molpharmaceut.8b00673

Hatogai, K. (2016). Molecular Subtypes of Gastric Cancer. Gan to kagaku ryoho. Cancer \& Chemotherapy, 43(3), 281-285. 
Hu, B., El Hajj, N., Sittler, S., Lammert, N., Barnes, R., \& Meloni-Ehrig, A. (2012). Gastric cancer: Classification, histology and application of molecular pathology. Journal of Gastrointestinal Oncology, 3(3), 251. doi:10.3978/j.issn.2078-6891.2012.021

Huang, Q., Cheng, Y., Chen, L., Mingzhan, D., Wang, Y., Xu, G., ... \& Zou, X. (2018). Low risk of lymph node metastasis in 495 early gastric cardiac carcinomas: a multicenter clinicopathologic study of 2101 radical gastrectomies for early gastric carcinoma. Modern Pathology, 31(10), 1599-1607. doi:10.1038/s41379-018-0063-1

Inokuchi, M., Fujimori, Y., Otsuki, S., Sato, Y., Nakagawa, M., \& Kojima, K. (2015). Therapeutic targeting of fibroblast growth factor receptors in gastric cancer. Gastroenterology Research and Practice, 2015. doi:10.1155/2015/796380

Ito, M., Takata, S., Tatsugami, M., Wada, Y., Imagawa, S., Matsumoto, Y., ... \& Chayama, K. (2009). Clinical prevention of gastric cancer by Helicobacter pylori eradication therapy: a systematic review. Journal of Gastroenterology, 44(5), 365-371. doi:10.1007/s00535-009-0036-8

Jomrich, G., \& Schoppmann, S. F. (2016). Targeted therapy in gastric cancer. European Surgery, 48(5), 278-284. doi:10.1007/s10353-016-0389-1

Joshi, S. S., \& Badgwell, B. D. (2021). Current treatment and recent progress in gastric cancer. CA: $A$ Cancer Journal for Clinicians, 71(3), 264-279.doi:https://doi.org/10.3322/caac.21657

Kharkar, P. S. (2020). Cancer Stem Cell (CSC) Inhibitors in Oncology-A Promise for a Better Therapeutic Outcome: State of the Art and Future Perspectives. Journal of Medicinal Chemistry, 63(24), 15279-15307. doi:10.1021/acs.jmedchem.0c01336

Kim, T. Y., Jong, H. S., Jung, Y., Kim, T. Y., Kang, G. H., \& Bang, Y. J. (2004). DNA hypermethylation in gastric cancer. Alimentary Pharmacology \& Therapeutics, 20, 131-142. doi:10.1111/j.13652036.2004.01984.x

Kosmider, O., \& Moreau-Gachelin, F. (2006). From mice to human: the "two-hit model" of leukemogenesis. Cell Cycle, 5(6), 569-570. doi:10.4161/cc.5.6.2577

Li, J. (2020). Gastric cancer in young adults: a different clinical entity from carcinogenesis to prognosis. Gastroenterology Research and Practice, 2020.doi:10.1155/2020/9512707

Ling, Y., Watanabe, Y., Nagahashi, M., Shimada, Y., Ichikawa, H., Wakai, T., \& Okuda, S. (2020). Genetic profiling for diffuse type and genomically stable subtypes in gastric cancer. Computational and Structural BiotechnologyJournal, 18, 3301-3308. doi:10.1016/j.csbj.2020.10.021

Liu, X., \& Chu, K. M. (2014). E-cadherin and gastric cancer: cause, consequence, and applications. BioMed Research International, 2014. doi:10.1155/2014/637308

Mallath, M. K. (2020). Gastric Cancer. Geriatric Gastroenterology, 1-52.

Mathers, C. D., \& Loncar, D. (2006). Projections of global mortality and burden of disease from 2002 to 2030. PLoS medicine, 3(11), e442. doi:10.1371/journal.pmed.0030442

Matsueda, S., \& Graham, D. Y. (2014). Immunotherapy in gastric cancer. World Journal of Gastroenterology: WJG, 20(7), 1657. doi:10.3748/wjg.v20.i7.1657

Meyerhardt, J. A., Tepper, J. E., \& Venook, A. P. (2015). Special series: advances in GI cancer. Journal of Clinical Oncology, 33(16), 1717.

Mezhir, J. J., Pillarisetty, V. G., Shah, M. A., \& Coit, D. G. (2010). Randomized clinical trials in gastric cancer. Surgical Oncology Clinics, 19(1), 81-100. doi:10.1016/j.soc.2009.09.011

Mustafa, M., Menon, J., Muniandy, R. K., Illzam, E. M., Nornazirah, A., Nang, M. K., ... \& Sharifa, A. M. (2017). Gastric cancer: risk factors, diagnosis and management. IOSR Journal of Dental and Medical Sciences, 16, 69-74.doi:10.9790/0853-1603126974 
Oliveira, C., Seruca, R., \& Carneiro, F. (2006). Genetics, pathology, and clinics of familial gastric cancer. International journal of surgical pathology, 14(1), 21-33. doi:10.1177/106689690601400105

Ong, C. K. S., Lirk, P., Tan, C. H., \& Seymour, R. A. (2007). An evidence-based update on nonsteroidal anti-inflammatory drugs. Clinical Medicine \& Research, 5(1), 19-34. doi:10.3121/cmr.2007.698

Parsonnet, J., Friedman, G. D., Orentreich, N., \& Vogelman, H. (1997). Risk for gastric cancer in people with CagA positive or CagA negative Helicobacter pylori infection. Gut, 40(3), 297-301. doi:10.1136/gut.40.3.297

Pizzo, L., Lasser, M., Yusuff, T., Jensen, M., Ingraham, P., Huber, E., ... \& Girirajan, S. (2021). Functional assessment of the "two-hit" model for neurodevelopmental defects in Drosophila and X. laevis. PLoS genetics, 17(4), e1009112. doi:10.1371/journal.pgen.1009112

Polk, D. B., \& Peek, R. M. (2010). Helicobacter pylori: gastric cancer and beyond. Nature Reviews Cancer, 10(6), 403-414. doi:10.1038/nrc2903

Postlewait, L. M., Squires, M. H., Kooby, D. A., Poultsides, G. A., Weber, S. M., Bloomston, M., ... \& Maithel, S. K. (2015). The prognostic value of signet-ring cell histology in resected gastric adenocarcinoma. Annals of Surgical Oncology, 22(3), 832-839. doi:10.1245/s10434-015-4724-8

Rampazzo, A., Mott, G. L., Fontana, K., \& Fagundes, R. B. (2012). Gastric adenocarcinoma trends in the central region of Rio Grande do Sul (Southern Brazil): what has changed in 25 years?. Arquivos de Gastroenterologia, 49, 178-183. doi:10.1590/S0004-28032012000300002

Rausei, S., \& Lianos, G. D. (2020). Treatment of gastric cancer. doi:10.3390/cancers 12092627

Rawla, P., \& Barsouk, A. (2019). Epidemiology of gastric cancer: global trends, risk factors and prevention. Przeglad Gastroenterologiczny, 14(1), 26.doi:10.5114/pg.2018.80001

Russell, R. I. (2001). Non-steroidal anti-inflammatory drugs and gastrointestinal damage-problems and solutions. Postgraduate Medical Journal, 77(904), 82-88. doi:10.1136/pmj.77.904.82

Saito, M., Saito, K., Nakano, H., Kikuchi, T., Okayama, H., Fujita, S., ... \& Kono, K. (2018). Annexin A1 is upregulated in p53-positive gastric cancer. Annals of Cancer Research and Therapy, 26(1), 26-30. doi:10.4993/acrt.26.26

Schipper, D. L., \& Wagener, D. J. (1996). Chemotherapy of gastric cancer. Anti-cancer Drugs, 7(2), 137149. doi:10.1097/00001813-199602000-00001

Sharma, R. (2021). Global, regional, national burden of breast cancer in 185 countries: evidence from GLOBOCAN 2018. Breast Cancer Research and Treatment, 187(2), 557-567.doi:10.1007/s10549020-06083-6

Shtygasheva, O., Ageeva, E., Maksimov, M., \& Strizhakov, G. (2010). The phenotype lymphocytes of patients withH. pylori-associated the gastric cancer: Poster no.: P7. 10. Helicobacter, 15(4).

Sitarz, R., Skierucha, M., Mielko, J., Offerhaus, G. J. A., Maciejewski, R., \& Polkowski, W. P. (2018). Gastric cancer: epidemiology, prevention, classification, and treatment. Cancer Management and Research, 10, 239. doi:10.2147/CMAR.S149619

Slomski, A. (2019). H pylori treatment may reduce long-term gastric cancer risk. JAMA, 322(20), 19481948.

Smyth, E. C., Verheij, M., Allum, W., Cunningham, D., Cervantes, A., \& Arnold, D. (2016). Gastric cancer: ESMO Clinical Practice Guidelines for diagnosis, treatment and follow-up. Annals of Oncology, 27, v38-v49.doi:10.1093/annonc/mdw350

Solsky, I., \& In, H. (2021). Surgical Treatment for Gastric Cancer. Gastrointestinal Endoscopy Clinics, 31(3), 581-605.doi:10.1016/j.giec.2021.04.001

Stanland, L. J., \& Luftig, M. A. (2020). The role of EBV-induced hypermethylation in gastric cancer 
tumorigenesis. Viruses, 12(11), 1222. doi:10.3390/v12111222

Suzuki, H., \& Matsuzaki, J. (2018). Gastric cancer: evidence boosts Helicobacter pylori eradication. Nature Reviews Gastroenterology \& Hepatology, 15(8), 458-460. doi:10.1038/s41575-018-0023-8

Tamura, G. (2006). Alterations of tumor suppressor and tumor-related genes in the development and progression of gastric cancer. World Journal of Gastroenterology: WJG, 12(2), 192. doi:10.3748/wjg.v12.i2.192

Thiel, A., \& Ristimäki, A. (2015). Targeted therapy in gastric cancer. Apmis, 123(5), 365372.doi:10.1111/apm.12359

serkan SENGER, A., GÜlMEZ, S., OFLUOGLU, C. B., Ayhan, Ö. Z., OMEROGLU, S., POLAT, E., \& DUMAN, M. (2020). Incidence and Prognostic significance of Signet Ring Cell Histology in Gastric Cancer. A cross sectional study from Turkey. Iranian Red Crescent Medical Journal, 22(9).doi:10.32592/ircmj.2020.22.9.34

Yang, S., Wang, C., Hu, Y., Yang, H., Wang, S., Zhou, B., ... \& Xie, X. (2021). Function of Non-coding RNA in Helicobacter pylori-associated Gastric Cancer. Frontiers in Molecular Biosciences, 8, 210. doi:10.3389/fmolb.2021.649105

Wang, J., Xiu, J., Baca, Y., Battaglin, F., Arai, H., Kawanishi, N., ... \& Lenz, H. J. (2021). Large-scale analysis of KMT2 mutations defines a distinctive molecular subset with treatment implication in gastric cancer. Oncogene, 1-12. doi:10.1038/s41388-021-01840-3

Woell, E., Devries, A., Eisterer, W., Hejna, M., Keil, F., Stein, H., ... \& Greil, R. (2008). Chemotherapy in gastric cancer. Anticancer Research, 28(2B), 1213-1219.

Won, E., \& Janjigian, Y. Y. (2015). Role of HER2 in Gastric Cancers. In Gastric Cancer (pp. 77-89). Springer, Cham. doi:10.1007/978-3-319-15826-6_5

Yusefi, A. R., Lankarani, K. B., Bastani, P., Radinmanesh, M., \& Kavosi, Z. (2018). Risk factors for gastric cancer: a systematic review. Asian Pacific Journal of Cancer Prevention: APJCP, 19(3), 591.doi:10.22034/APJCP.2018.19.3.591

Zhang, J., Jiang, M., Li, S., Zhang, Z., Sun, H., Yang, F., \& Liang, H. (2021a). Developing a Novel Anticancer Gold (III) Agent to Integrate Chemotherapy and Immunotherapy. Journal of Medicinal Chemistry. doi:10.1021/acs.jmedchem.1c00050

Zhang, X., Liu, F., Bao, H., Wang, A., Han, M., Wu, X., ... \& Zheng, L. (2021b). Distinct genomic profile in h. pylori-associated gastric cancer. Cancer Medicine, 10(7), 2461-2469.doi:10.1002/cam4.3765

Zhu, Y., Zhu, X., Wei, X., Tang, C., \& Zhang, W. (2021). HER2-targeted therapies in gastric cancer. Biochimica et Biophysica Acta (BBA)-Reviews on Cancer, 188549. doi:10.1016/j.bbcan. 2021.188549 


\title{
Bio-Inks for 3D Bioprinting: A Scientific Analysis of Two Decades of Progress, Challenges and Future Prospects
}

\author{
Sritama Chatterjee, Sayak Pan, Suchandra Chatterjee* \\ Department of Chemistry, Surendranath College, Kolkata, India \\ ${ }^{*}$ Corresponding Author's Email: chatterjeesuchandra01@gmail.com
}

\begin{abstract}
The overall interest for organ replacement and tissue regeneration is gradually expanding consistently with time. The advancement in tissue engineering and regenerative drug production utilizing 3D bioprinting have succeeded to regenerate broken organs and tissues into perfect ones. The essential material for this type of printing is Bio Ink, that is a must for creating practical organs and tissue structures. However, the bio inks thus used in 3D printing technology need to satisfy certain properties that are critical for their use in this regime. Combination of various techniques and enhancement in properties of these bio inks are essential for developing more and more efficient bio inks in future as per demand. Present review consists of a thorough study on various modern state-of-art polymer-based bio inks, commonly utilized in 3D bioprinting sector. Several types of bio ink formulations starting from cell-biomaterial based bio inks to cell-based bio inks like tissue spheroids and cell aggregates so far have been used for tissue engineering and regenerative drug production. Additionally, tunable bio inks, that are compatible with printable live cells, and sufficiently stable are now-a-days also evolving using chemically composed biomaterials with proper mixing of cells and hydrogels. Hope the current review would succeed to highlight large potential of the most modern bio inks, that are desired for more advanced tissue/organ structure generation using 3D bioprinting in near future.
\end{abstract}

\section{Keywords: Bioprinting; Bio ink; Tissue; Cell; Organ; Biomaterial; Scaffold; Hydrogel}

\section{INTRODUCTION}

Bio fabrication is a rising field that includes formulations of tissue structures with a definite planning. Typical bio fabrication techniques include various steps like desiccation, electrospinning, microengineering etc. Though various fabrication techniques are available there in generating threedimensional (3D) structures with a variety of biomaterials (Gungor-Ozkerim et al., 2018), they often possess restricted flexibility in the fabrication methodology. Modernist definition of bio fabrication is creation of helpful biological products like micro-tissues, hybrid cell-materials etc. via bio assembly, bioprinting, and consequent tissue maturation processes in a computerized way using living cells, cell aggregates, and variety of bioactive molecules. As of late, this 3D bioprinting has arisen as a distinctive bio fabrication methodology with significantly improved reproducibility powered by the machinecontrolled deposition methods. Fundamentally, bioprinting method licenses for the manufacture of 3D tissue constructs having pre-customized designs and geometries using biomaterials or potential living cells as bio ink by contemporizing its deposition through mechanized maneuvers. Laser-assisted bioprinting, inkjet/droplet bioprinting, and extrusion-based bioprinting are the three most popular 3D bioprinting methods available. Coincidental or consequent printing of multiple materials are also possible by using modern multi-head deposition system. For bioprinting, at the very outset, 3D constructs (to be manufactured) are first programmed with a computer-aided design/computer-aided manufacturing (CAD/CAM) system and then the requisite structure is actually made using proper bio inks. These bio inks are vital elements in 3D bioprinting that actually get cross-linked and stabilized by the bioprinting process to produce the desired tissue structure. The right choice of bio ink depends primarily on the target tissue structure and also on the type of bioprinter used. Despite enormous potential, applications of bio-printing technology are till date restricted for lack of acceptable bio inks, 
required for meeting minimum requirements of bioprinting to own correct bioactivity of various cells. A perfect bio ink got to own some desired mechanical, physical, chemical, and biological properties leading to: (i) adequately mechanically strong tissue structures, (ii) gelation and stabilization processes that are adjustable for bioprinting of flexible structures, (iii) biocompatibility and biodegradability that is capable of mimicking natural microenvironment of the tissues, (iv) chemical modifications to satisfy tissue-specific needs, and (v) large-scale production with minimum batch-tobatch variations. However, all possible bioprinting applications desperately need standardized procedures for bio ink formulation. The main motto of this review is to gift all the readers an in-depth summary of current scenario of existing bio inks. It would also include both natural and artificial biomaterials used either alone or together. The studies in which biopolymers are printed with bio inks made of embedded cells (Mandrycky et al., 2016) would also be discussed here. The review would initially describe natural bio inks, then gradually move towards artificial bio inks and cellular spheroids, followed by some commercial bio inks, their potential uses and flaws. A brief discussion would also be there on various new bio ink formulation techniques and related challenges.

\section{LITERATURE REVIEW AND DISCUSSION}

\section{3D Bioprinting:}

In the field of regenerative medicine 3D bioprinting is an emerging field that can produce cell-laden 3D structures having the capacity of mimicking body tissues playing a crucial role in drug delivery and cancer studies apart from tissue engineering. Bioprinting can generate case-specific spatial geometry with controllable microstructure, and proper positioning of different cells for fabrication of tissue engineering. In this review, the diverse manufacture procedures would be delineated first and would be discussed shortly along with their benefits and challenges. Then Bio inks would be discussed in detail just as ebb and flow of research in this field. We can find nozzle-based techniques such as inkjet printing, extrusion printing, and laser-based ones, all have the capacity of generating the desired bio printed scaffolds using appropriate bio inks.

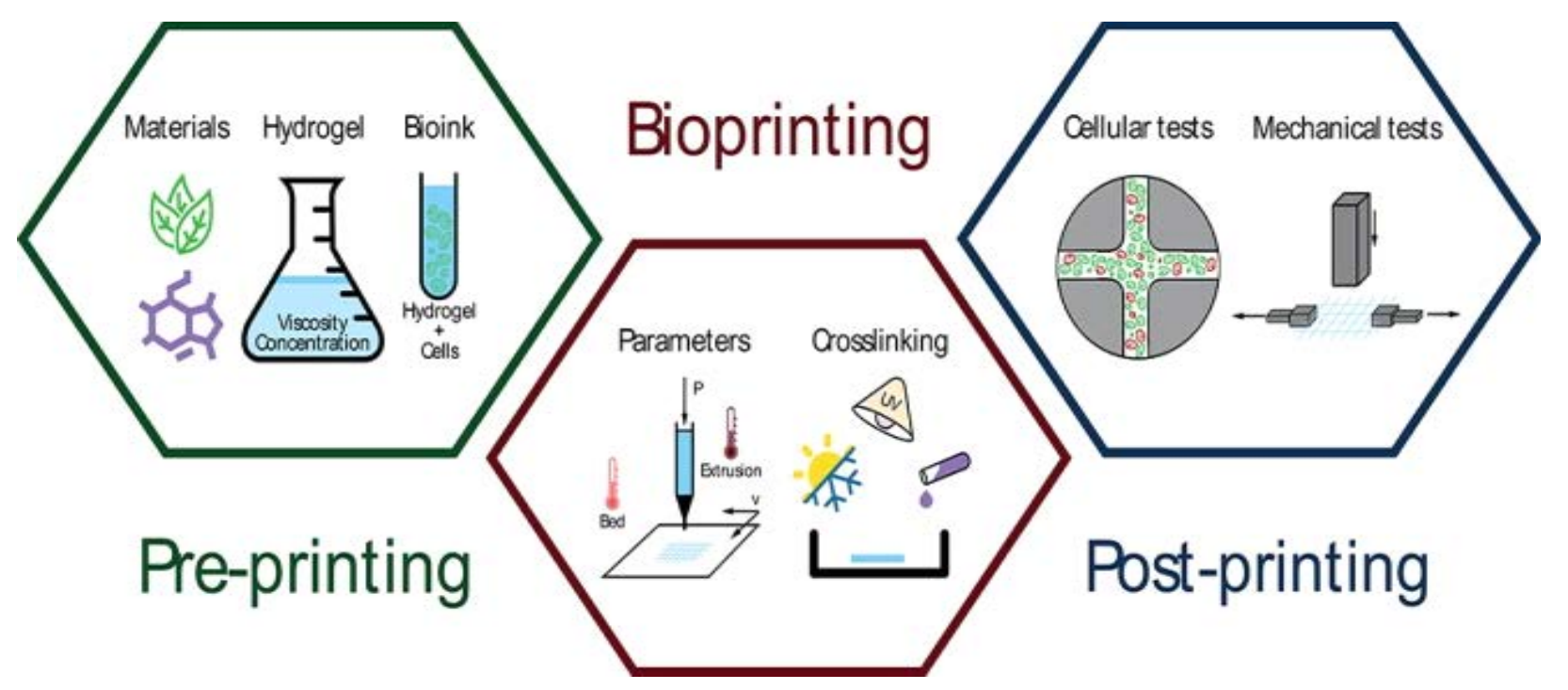

Figure 1: Process of Bioprinting

All the formulation techniques were found to effectively affect the cell viability, structural resolution, and speed cum precision of printing. In addition, material choice and their respective concentrations were also found to affect the quality of printing (Hong et al., 2018). Every single procedure has its own discrete individual benefit and pitfall and hence we should combine multiple techniques to consolidate the upsides of every strategy. Bioprinting employs the first principle of 3D printing for generating medical specialty elements with employment of biocompatible materials. Such materials are called Bio ink. They should in addition contain bioactive components like live cells and growth factors. 
Developing novel bio inks is one amongst the nice and cozy topics of current day research. 3D bioprinting being triple crown of printing sensible tissues and organs can also create nice influence on regenerative drugs. This bioprinting is done through quite a range of techniques like inkjet, extrusion, and laser/light varieties. Inkjet bioprinters can manufacture notably high-resolution patterns (Vijayavenkataraman et al., 2018) by generating little ink droplets on a substrate, that typically needs liquid bio inks. Similarly, laser-based bioprinting also needs liquid-like bio inks. Whereas extrusionbased bioprinting needs bio inks with certain viscousness as this method uses a mechanical force to drive the material via a nozzle in an exceedingly managed manner to construct 3D structures. Inkjet, extrusion, and laser-assisted bioprinting are all used for 3D bioprinting. Different other techniques of bioprinting, like Freedom Reversible Embedding of Suspended Hydrogels (FRESH) are also utilized intelligently for tissue regeneration. Stereolithography is another method that has been adopted for bioprinting. A lightweight stereolithography-based low-cost 3D bioprinter can perform point-by-point curing process that can print mixed cells explicitly with good biocompatibility. It can safely be used for screening of drugs, studying various pathological mechanisms, and preparing biological disease models. Lightweight bioprinting is that vital drive like the laser-assisted one which by choice solidifies a bio ink layer wise additively to build up the desired objects. "Continuous liquid interface production" developed by Tumbleston et al., (2015) is the most modern technique developed in this field that can astonishingly improve the printing quality and time. Although that methodology is rarely used except for printing some difficult and complicated tissue architectures. In this review, the authors would mainly concentrate on bio inks used in various techniques of bio printing, like Pressure-Assisted, ThermalAssisted, Inkjet, Extrusion based, Magnetic-Assisted, Light-Assisted and Micro-fluidics Bioprinting.

\section{Bio-inks:}

The main constraints of bioprinting always involve choice of right biomaterials to be utilized as bio inks. Biomaterials whether natural or artificial play an integral role in drug designing (Sun et al., 2020) and are utilized effectively in medical applications to support, enhance, or replace broken tissue or a biological system.

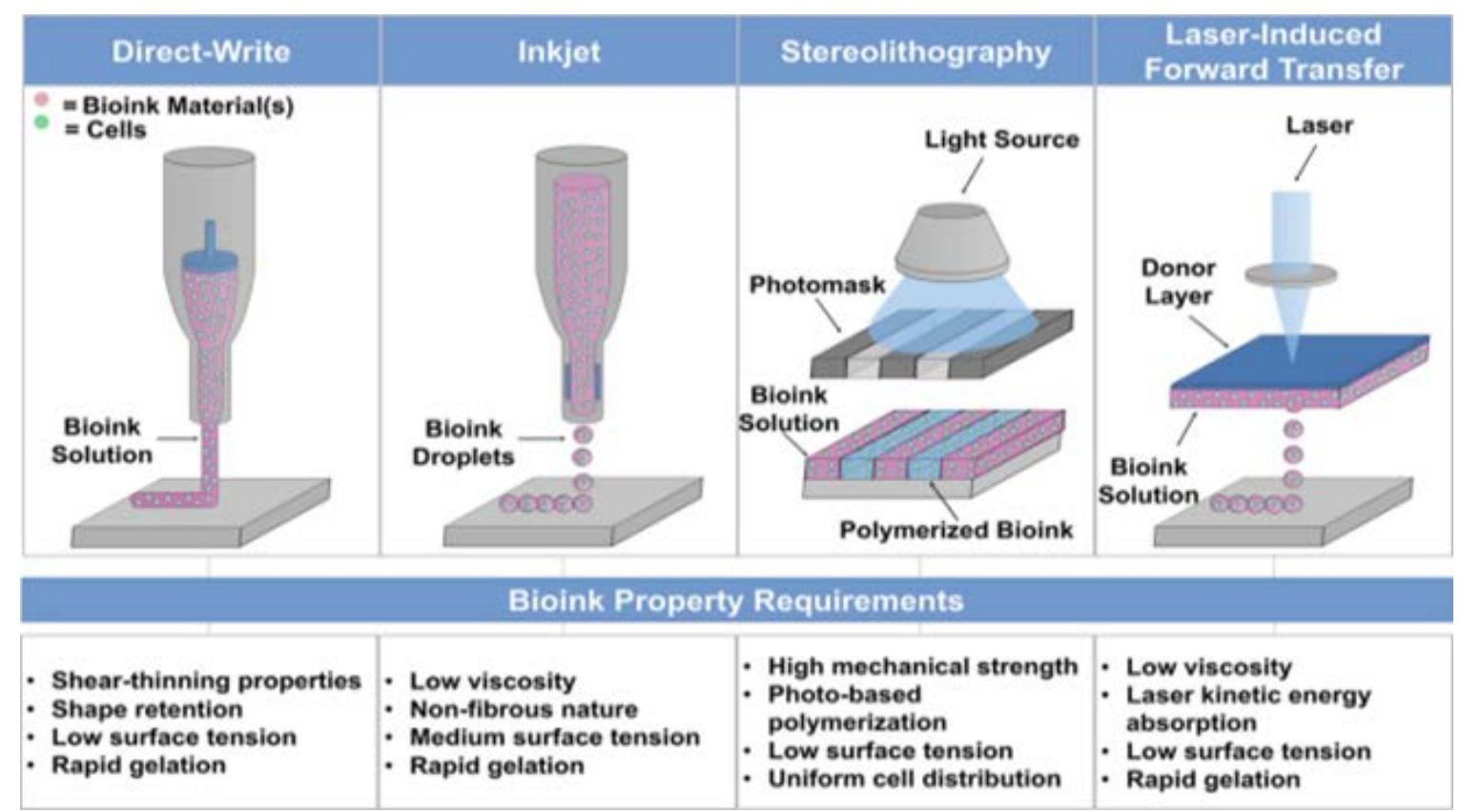

Figure 2: Different Bio Inks

Biomaterials are generally composed of cells only, however in some cases, an extra carrier material encapsulating the cells is additionally present. This carrier is typically a biopolymeric gel, acting as a 3D molecular scaffold. The cells present get attached to this gel that allows them to unfold, grow and proliferate. Hydrogels are the mostly used bio inks. Pre-fabrication crosslinking of the precursor hydrogel solutions to lift it to a state of higher viscosity, followed by post-fabrication cross-linking is 
most desired. Bio inks can embody cells in various forms like single cells, aggregated cell spheroids, cellular rods, mini-tissues or organoids, silk coated cells, cells microcarriers seeded with cells, or encapsulated cells in mixed microenvironment. Here, in this short review, authors would try to briefly summarize some of the most used natural and artificial polymers that can be efficiently used as bio inks.

(a) Thermoplastic Polymers: A portion of the conventionally utilized biomaterials are thermoplastic polymers in view of their innate flexibility, adaptability, and adjustable habitual properties. Resorbable thermoplastics are one amongst the primary sort of materials that were developed for medical applications. This category of materials has found use in several applications like surgical anchors, screws, pins, and implants. Polycapro-lactone (PCL) (Murphy, De Coppi \& Atala, 2020) could be a perishable polyester that has found wide application in the medical field. PCL is hydrophobic and semi crystalline in nature. This thermoplastic has excellent flexibility in drug delivery and as a system material for bio printed constructs. Polylactic acid (PLA) based thermoplastics are ordinarily used within biomaterial field being approved by Food and Drug Administration (FDA) (Hospodiuk et al., 2017) because of its degradability, processability, and biocompatibility. Explicit application of thermoplastics as a support material for protecting softer hydrogel-based materials inside the body demands proper blending of PLA with materials like nanohydroxyapatite and carbon nanotubes. Once incorporated into a block co-polymer with PLA, the degradation mechanics, property, crystallinity, and stiffness are simply tailored. This category of materials has found their usage in drug delivery and tissue engineering in low mechanical stress environment.

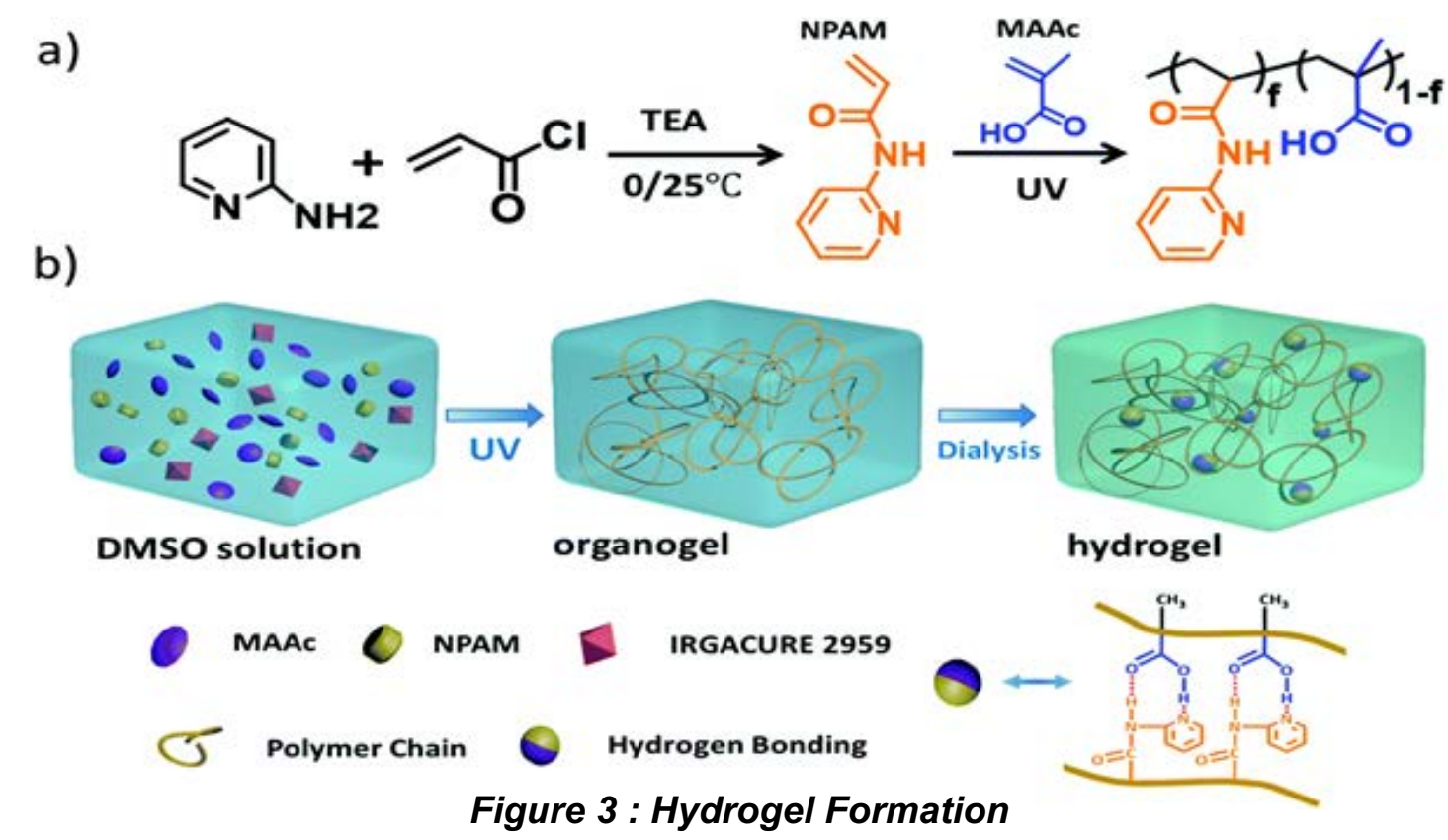

(b) Hydrogels: Hydrogel is a network of crosslinked compound chains that are deliquescent, typically found as a colloid in which water is the dispersion phase. The cross links that bond the polymers of a hydrogel can be of two broad categories (Hölzl et al., 2016) physical and chemical. Physical cross links contain gas bonds, hydrophobic interaction, and chain entanglements. Thanks to these cross links and structural integrity, colloidal gel network doesn't dissolve much in water, rather they are extremely absorbent. Employment of hydrogels in imprinting has appeared as an active domain of analysis in the last half decade. Although they are ideal materials for cell culture and encapsulation, nonetheless their and hydrous states throw challenges to sound reproduction of planned constructs. In general agarose or albuminoids are employed as the "3D papers" or support and polyethylene glycol diacrylate (PEGDA) is used to print cell encapsulated gels. Induced gelation of alginate with photopolymerizable gels like Gelatin Methacrylate (GelMA) and PEGDA (Gillispie et al., 2020) conjointly has now a days become one of the most popular bio inks. Currently, varied bio printable hydrogels have arrived each with specific targeted aims and options. Natural polymers like alginate, chitosan, hyaluronic acid (HA) 
and gelatin after slight modification can also show smart printability, despite restricted tailoring ability. Artificial hydrogels like polyglicidol and polyethylene glycol (PEG) have shown wonderful chemical properties and mechanical tailoring ability. Chemical modification of natural polymers like HA, alginate, gelatin etc. tailored by chemists to formulate hydrogels has tried to break the demarcation between artificial and natural polymers. Current trend of making multicomponent inks (combos of synthetic/natural/modified materials) can be very useful in near future. Hydrogels usually need a cross-linking step post or throughout bioprinting and might involve light chemical processes and ionic cross-linking for that and this cross-linking of hydrogels via novel methods has really become a promising field recently. Self-healing and shear-thinning hydrogels however, now a days have come into picture that generally do not require any further cross-linking steps. All these approaches have succeeded to form new hydrogels with definite edges in tailor ability and functionality over the natural materials.

(c) Resins: They are usually viscous substances that can be converted into rigid polymers through specific procedures. Though resins are naturally occurring substances, they can be created synthetically too. Some of these synthetic artificial resins have properties like natural plant resins while many of them are completely different. Resins belong to a special category of photo-cross linked biomaterial inks using various techniques like stereolithography (SLA), digital light processing (DLP) and 2-photon polymerization (2PP) (Ribeiro et al., 2017). These cross linkable resins (light assisted) are extensively used in bio fabrication, spanning from thermoset-like materials to soft and hydrated hydrogels. Upon irradiation with UV light, these resins form 3D network. Methacrylate resins as well as GelMA and PEGMA are mostly used for printing of hydrogels and polyesteracrylates for printing of thermosets. DLP method enables massive scale fabrication (Wang, Kelley \& Venditti, 2016) of light evoked thiolene resins, polyester acrylates, plastic fumarate resins etc. Cross-linkable resins prepared with ultraviolet light have an oversized variation of properties, depending on the type of the monomers used, density of structural network, light exposure, and secondary cross-linking steps, starting from hydrous hydrogels to useful resins. However, using lower energy light sources, modern techniques allow fabrication of bio ink resins minimizing harmful effects of cytotoxic and mutagenic UV light. Proteome studies envisage that UV cross linkable materials, cells show no discernible injury to their proteome.

\section{Biomaterials for Bio Inks and their Properties:}

As discussed earlier, bio inks employed for 3D bioprinting ought to possess some vital qualities like smooth printability, characteristic mechanical properties, modifiable functionality, controlled

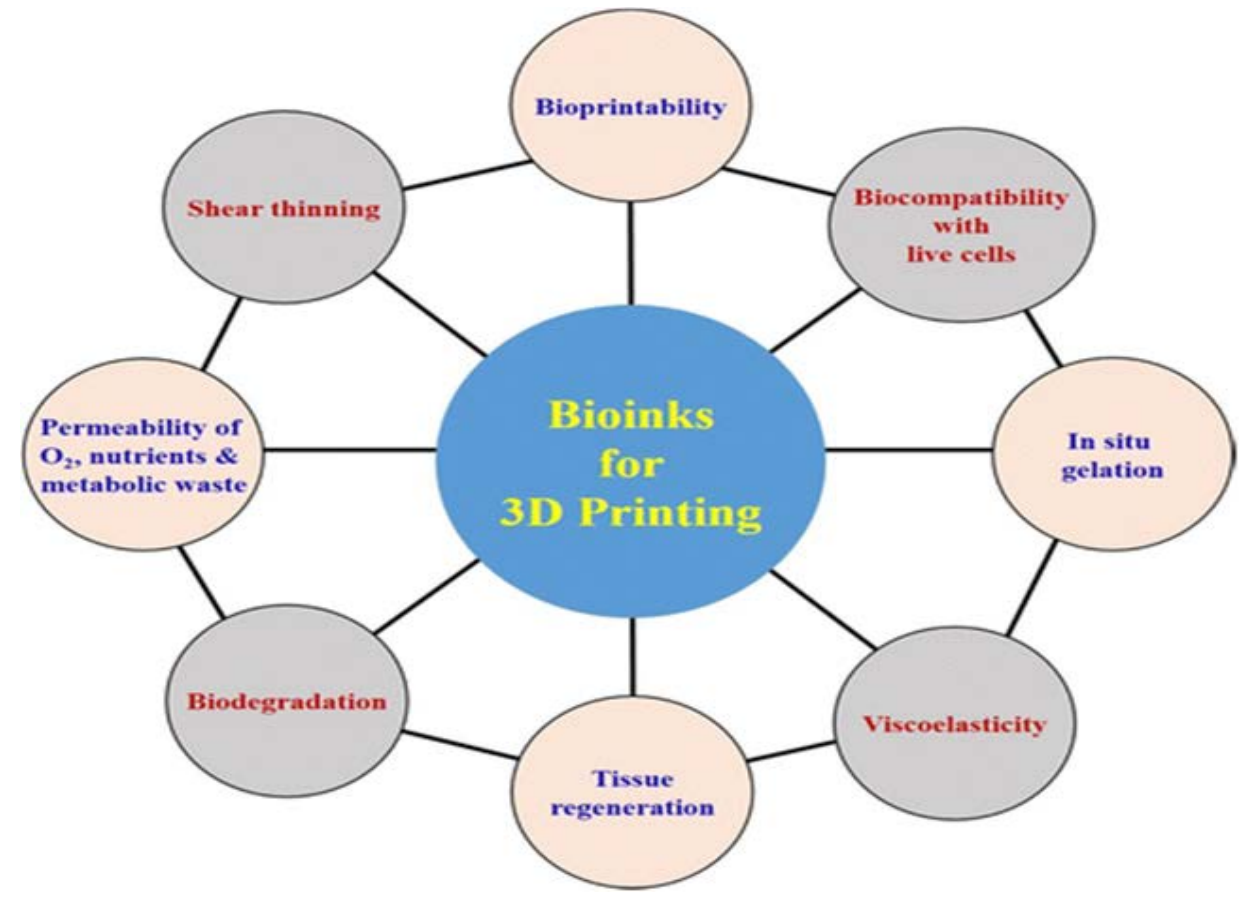

Figure 4 : Properties of Bio inks 
biodegradability, and non-toxicity to target cells. Proper bio ink ought to be selected to regenerate desired tissue structure or organs. Various biomaterials so far have been reported as bio inks (Mirski, Bekhta \& Dziurka, 2019) and natural biomaterials obtained from natural resources have variable benefits over artificial ones. However, artificial polymers also possess their own unique properties that are absent in natural polymers like tunable mechanical stability, photo crosslinking capacity and so forth.

\section{(i) Natural Bio Inks}

(a) Agarose-based bio inks: The marine saccharide Agarose, prepared from algae, is one of the most popular biopolymers due to its amazing gel forming ability. Agarose is a linear chain compound with repetitive agaro-biose units with a backbone made up of disaccharides like D-galactose and 3,6anhydro-L-galactopyranose (Wang, Kelley \& Venditti, 2016). However, its capacity to help in cell development is limited, albeit its impressive gelation, mechanical and biocompatibility properties. Hence, blends of purposeful biomaterials together with the agarose gel became quite popular. In 2017 Kreimendahl and his team separately reported employment of agarose-based bio ink consisting of both collagen and fibrinogen. Flexibility of these agarose-based mixed biomaterials to create stable 3D structures and support epithelial tissue and fibroblast cell growth was meticulously reported by them. In 2018 Yang's group used agarose/collagen in conjunction with $\mathrm{Na}$ alginate for bio ink production for animal tissue engineering applications. Biomaterials thus printed showed increased mechanical properties along with their superior gelling capability. In $2017 \mathrm{Gu}$ and his team succeeded in developing useful neurons and nerve cells from pluripotent stem cells utilizing agarose along with alginate and carboxymethyl-chitosan (CMC). Chemically modified agarose like carboxylated agarose was also reported to be used as bio inks for formulating automatically tunable 3D tissue constructs. Human Mesenchymal Stem Cells (hMSCs) were also used in some studies yielding terribly high cell viability compared to native agarose gel. Degree of carboxylation could be altered (Huang et al., 2017) to get different gels with varied mechanical properties as per the demand. Kucukgul and his team (2013) reported use of cellular aggregates for direct cell 3D printing with sleek muscle, epithelial tissue, and fibroblast cells. Flexibility of those constructs to fuse with each other to produce higher cell viability was also demonstrated by them. Although these type of bio inks show smart gelation properties, chemical modifications and appropriate mixing are often needed for supporting the resultant 3D printed structures and for boosting a lot of cellular functions as well. In 2017 Jang and his team successfully pointed out that a suitable combination of agaroses having both high and low relative molecular masses can actually enhance its ability to make 3D structures as support towards live cells. Fibroblast cell usage in in-vitro studies proved 2:1 (high: low) quantitative mixture to be most sensible and suitable for bioprinting in soft tissue engineering.

(b) Alginate-based bio inks: Alginate, the charged polysaccharide is also a natural biopolymer obtained from algae, often named as alginic acid or algin. The Alginate compounds (Norouzi, Nazari \& Miller, 2016) are mostly made up of have two monomers: (1-4)- $\beta$-D-mannuronic acid and $\alpha$-Lguluronic acid. The first one helps in gel formation, while the second one and their mixtures help in inducing flexibility to the fabric. These biopolymers can effectively entrap water and different other molecules due to capillary action enabling them to diffuse inside out and thereby making them most efficient bio-inks. Thus, in $2014 \mathrm{Xu}$ with his group used these alginate-based bio inks along with animal tissue cells for printing hollow constructs, in which the special vessel-like microfluidic channels can effectively transport chemical element, nutrients, biomolecules etc. ultimately support cell growth. In a similar study, Yu and his group (2016) also tried alginates along with animal tissue cells as bio inks for developing cannular constructs with a triaxial nozzle assembly. Huang and his team (2017) next discovered a co-axial system of alginate-based colloidal gel material that might print strong 3D constructs having micro-channels for effective nutrient delivery. In 2015 Christensen and his team reported a metal alginate bio ink using mouse fibroblast cells for construction of vascular-like structures using salt crosslinker (Wolf et al., 2012). Similarly, various polymers were homogenized with alginate, like PCL, poloxamer, hydroxyapatite, gelatin etc. to fabricate numerous desired 3D constructs. Alginates are best utilized to foster 3D neural tissues. Using alginate-based bio inks, human embryonic stem cells could be bio printed for the first time. Yang with his group (2018) succeeded to construct a 3D tissue model of cervical tumor using Hela cells and a mix of bio inks 
consisted of gelatin, alginate, and fibrinogen for in vitro study. In 2017 Ahlfeld and his team mixed artificial nano silicate clay with alginate and $\mathrm{CMC}$ to formulate two different bio inks. This nano silicate clay incorporation further enhanced the power of the alginates and CMC samples (Ono, 2017) to unleash loaded medicine in a slowly paced sustained manner. Alginate-based and hyaluronate-based nano fibrillated polysaccharide composite bio inks were compared for formulating animal tissue constructs using pluripotent stem cells in another study. A separate study reported the use of alginate in combination with different artificial polymers like Poly (Ethylene Glycol)-Tetra-Acrylate (PEGTA) and GeIMA for developing the desired 3D bio printed materials. Addition of PEGTA helped significantly in bioprinting of advanced multilayer hollow 3D systems like stable tube-shaped structures. In future this method might help in getting more and more sophisticated vascularized tissue constructs for various tissue engineering applications. Thus, all these studies emphasized on varied benefits of alginatebased bio inks over the traditional hydrogels.

(c) Collagen-based bio inks: Collagen obtained from natural biomaterials is the main element of ECM. It is used as a bio ink material either alone or in a combined state due to its amazing biocompatible properties. They can be crosslinked by modifying temperature or $\mathrm{pH}$ and by using vitamin B. Collagen crosslinking always provides redoubled strength and visco-elastic properties compared to the non-crosslinked scleroprotein. Direct usage of these scleroproteins (So \& Xia, 2019) in $3 \mathrm{D}$ printing is quite difficult and hence use of different alternative gelation materials become essential. In 2018 Yang and his group recommended the use of a mixture of scleroprotein and alginate for cartilage tissue regeneration. In a similar work, combination of scleroprotein with gelatin was exploited utilizing Drop on demand (DOD) inject technique to co-culture of human epithelium cells and hMSCs. The power of usage of mixed bio inks for constructing stable 3D constructs with high biological activity and rheologic properties was clearly established. A new collagen-based bio ink was also reported by Yeo et al., (2016). Their results showed redoubled mechanical and biological properties (López-Marcial et al., 2018) with introduction of scleroprotein. They used scleroprotein as the core biomaterial and alginate as sheath biomaterial along with human stem cells in their study. A similar work demonstrated employment of scleroprotein with alginates crosslinked by polyphenol. Bio inks consisting of human fat stem cells showcased higher cell viability and proliferation compared to alginate-based bio inks after printing. A recent work has proved the power of transglutaminasecrosslinked gelatin-based bio-printed 3D constructs in developing vascularized structures. These structures are generally useful in constructing complicated tumor models and in various fields of tissue engineering.

(d) Hyaluronic acid-based bio inks: The natural ECM Hyaluronic acid (HA) abundantly seen in cartilages and connective tissues are often employed in 3D bioprinting for formulating 3D structures. Various types of HA-based bio inks have been reported till date. An important study used photocrosslinked HA (Mirdamadi et al., 2019) as bio inks to get enhanced physical properties. Like allnatural polymers, HA also has low mechanical properties and slow gelatinous behavior, compared to all artificial hydrogels. Very recently Ouyang and his team using secondary crosslinking methodology has successfully created HA-based stable 3D printed constructs that resulted in sensible cellular adhesion properties that could be further enhanced by addition of oligopeptides within the hydrogels. Another research revealed how HA-based hydrogels by reacting with methacrylate can show high osteogenic properties (Fan et al., 2016). Methacrylate cluster here intelligently uses the photocrosslinking mechanism to enable the hydrogels to get cross-linked. Some recent studies revealed that combination of HA with different artificial polymers help in generating sufficiently stable structures with considerable cell viability. Chemical and photo-crosslinking are employed only to boost the useful properties of these bio inks. Various mixtures of different artificial polymers (Axpe \& Oyen, 2016) and their efficacy as injectable gels were sincerely investigated. Varieties of cells could be bio printed by employing them all. In 2018 Sakai and his group reported HA-gelatin based bio inks that may be polymerized even using visible radiation with the aid of Ruthenium-based complexes. In a separate work, extremely tunable HA-CMC combination gels were formulated. Variation of mechanical properties and cell viability with varying concentrations suggest that higher concentration might yield higher cell viability and impart sufficient stability to the printed 3D constructs. All such studies as a whole manifest the benefits of HA as bio inks in 3D bioprinting technology. 


\section{(ii) Artificial Bio Inks}

Apart from naturally available biomaterials, numerous types of artificial bio inks based on fibrin, cellulose, silk, ECM, cell aggregates, cell spheroids, etc. are also used in 3D printing.

a) Fibrin-based bio inks: Fibrin, generally seen inside blood helps in coagulation. Fibrin colloids are generally created out of fibrinogen due to catalytic treatment of thrombin possessing fantastic biocompatibility and biodegradability despite weak mechanical properties. Hence these bio inks are not much effective.

b) Cellulose-based bio inks: Carboxy Methyl Cellulose (CMC), the semi flexible saccharide can be obtained from cellulose. Environment-friendly colloidal gels can be obtained from this CMC by fixing its concentration, mass, type of salts, and the number of methyl groups attached. A CMC solution was found to form gels below $37^{\circ} \mathrm{C}$ and $\mathrm{CMC}$ based bioactive glasses were used to regenerate $3 \mathrm{D}$ bone structures. Production of a nanocellulose-alginate-based bio ink for its effective use in tissue engineering was reported by Markstedt and his group (2015). In another work nanocellulose hydrogels (Piras, Fernández-Prieto \& De Borggraeve, 2017) could formulate patient-specific auricular gristle tissues that manifested wonderful retention of form, size and cell viability. Polysaccharide nanofibrils and cross-linkable xylan-based bio inks also showed high mechanical integrity and amazing printing properties. In some studies, alginate based nanocellulose showed higher cell viability compared to their HAanalogues.

(c) Silk-based bio inks: Silk fibroin, the natural macromolecule is obtained from silkworm. These silkbased scaffolds are mostly utilized in the field of regenerative drugs and tissue engineering due to some of their special properties. In 2015 Das and his group prepared a silk-gelatin based bio ink using sonication and enzymatic crosslinking. A different work successfully showed how silk and gelatin as bio inks can typically enhance biocompatibility, cell permeability and tissue integration in soft tissue reconstruction, using glycerin as the physical cross linker. Alginate based silk fibroin macromolecules were also used as bio inks in Inkjet printing, cross-linking the tyrosine residues with peroxidase. Xiong and his team (2017) showed efficaciousness of primarily gelatin-silk based inks to regenerate skin tissues. In 2018 Zheng and his team reported silk-based bio inks made up of PEG exhibiting excellent high-resolution printability (Dzobo, Motaung \& Adesida, 2019). Recently, spider silk is also additionally receiving a lot of attention attributable to its amazing mechanical properties. DeSimone (2020) used recombinant spider silk proteins in a separate study for preparing bio inks. Even if printed constructs showed lesser cell viability using spider silk-based bio inks, once gelatin was added, the results were promising. For improving and enhancing these cell viability, biocompatible materials were added to silk to enhance the standard of printed materials.

(d) Extracellular matrix (ECM)-based bio inks: ECM is a mixed framework consisting of various parts like collagen, glycosaminoglycan, chondroitin sulfate, elastin, etc. along with cells. Decellularized ECMs ( $\mathrm{dECM}$ ) can be obtained from specific tissues wherefrom cells are removed by a stepwise procedure keeping the ECM intact that can be ultimately crushed to create a powder-like state and dissolved in appropriate solvents to prepare bio inks. Printability of these dECM-based bio inks (Shin et al., 2019) can be enhanced by addition of different hydrogels like PCL obtained from different tissue varieties. Researchers have developed a system which might exactly manage the temperature and $\mathrm{pH}$ of the bio inks to create gels even at $37^{\circ} \mathrm{C}$ while printing. It was shown by them that precise stacking of such cells by the system cannot succeed to impact the cell viability even with heating, to incite any harmful impact to the printed cells. 3D printing with dCEM along with dual stem cells was reported for internal organ patch development (Ali et al., 2019). Although dECM was found to provide sensible cell viability and functionality, isolation and quantification of DNA and ECM constituents from specified tissues, they are too expensive compared to other alternate gel-bio ink formulations used in 3D bioprinting.

(e) Cell aggregates as bio inks: Bio inks consisting of spherical cell aggregates (spheroid) containing thousands of cells are generally required to develop 3D printed constructs. Such spheroids are serially distributed by self-assembly method into biocompatible scaffolds. Yu and his group (2016) showed a completely unique bio ink made up of tissue spheroids for developing 3D constructs not hampering 


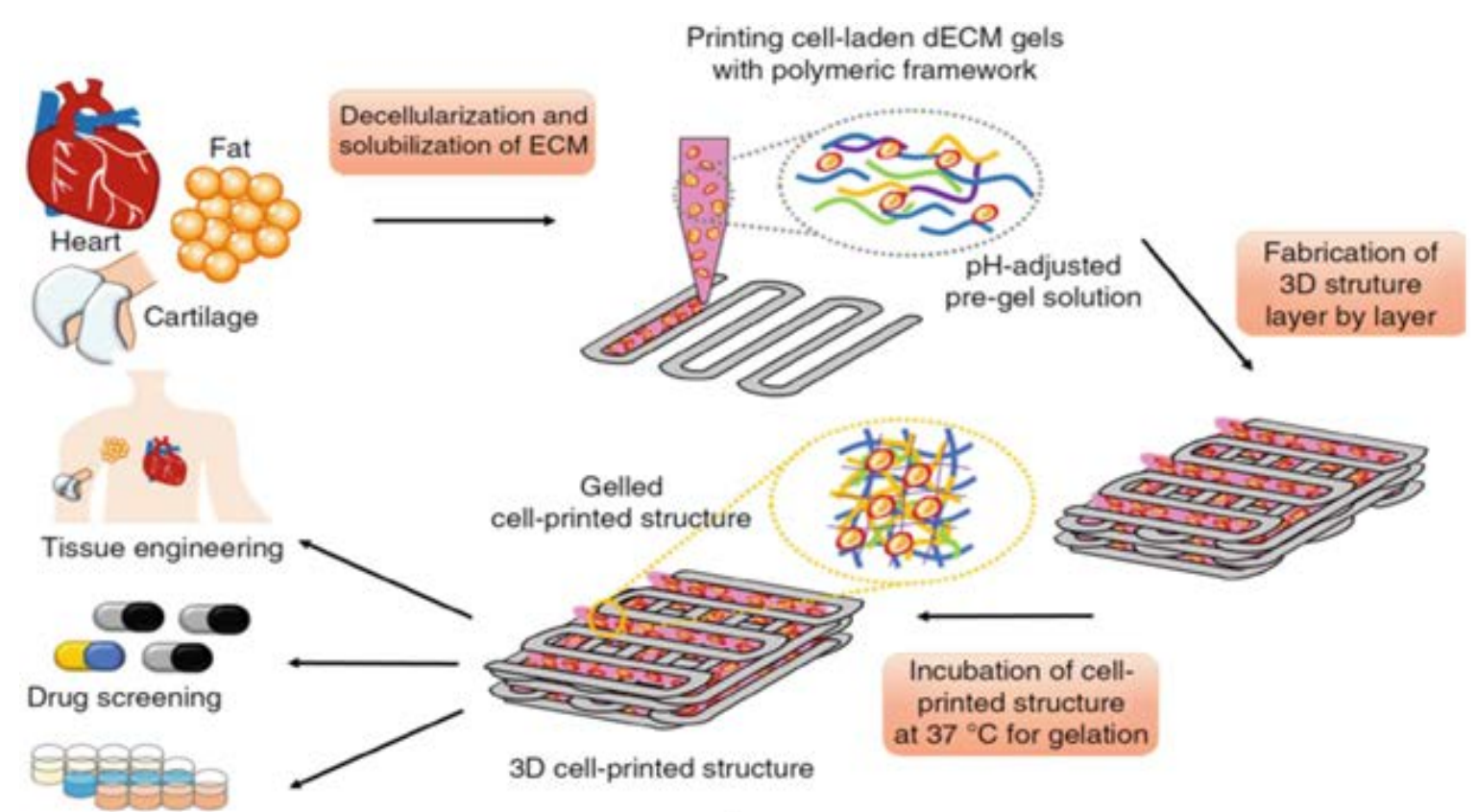

Figure 5: (ECM)-Based Bio inks

any scaffold. Tissue strands up to eight $\mathrm{cm}$ long could be developed by speedy fusion of cells utilizing self-assembly method (Bakirci et al., 2017) despite using any harsh chemicals as crosslinker or support material. Cell aggregates or cell sheets could also be formed employing some thermosensitive compound gels like Poly ( $\mathrm{N}$-isopropyl acrylamide) as substrate. When spontaneous cell growth starts onto the substrate, the cell sheets can be made detached by application of gentle heat not distressing the arrangement of the cell-matrix. Those detached cell sheets after getting completely separated can safely be used as potential bio inks for 3D bioprinting. Bio inks of this type displayed better effectiveness than the traditional cell aggregates for carefully preserving the ECM flawlessness.

\section{Challenges and Future Perspectives:}

Although 3D bio-printing has solid capacities to create the desired tissue and organ structures effortlessly, it desperately needs enhancement of various properties of bio inks and inevitably effective commercialization of the 3D printed products. This technique can promote the scope of development of more advanced case-specific 3D structures for medical emergencies. Among various other available techniques, the cell-laden hydrogels are most popular used for developing these 3D constructs. Choice of proper bio inks varied accessible bio inks and their properties have been discussed throughout this review. Though ideal bio ink preparation is yet to achieve, because of its gigantic commitments in various fields, it can possibly be utilized in industrial applications too over the long haul. Despite the fact that cell laden bio inks are normally utilized, ECM-based bio inks, decellularized bio inks, cell aggregates or spheroids likewise show promising outcomes towards manufacture of useful tissues or organs utilizing this 3D bioprinting innovation. Nonetheless, this load of strategies needs enormous number of explicit cells that typically restricts use of this 3D bio imprinting in different cases. Advent of advanced high resolution and low cost bioprinters may help to solve that problem enhancing the prospects of this specific area. Numerous novel polymeric biomaterials with supramolecular reasonableness, reversible crosslinking and stimuli responsive hydrogels have been accounted for as of late. Eventual fate of bio inks and 3D bioprinting is hence exceptionally encouraging, prompting manufacture of cutting-edge case-explicit tissue/organs and gadgets over the long haul.

\section{CONCLUSION}

Bioprinting is no doubt a rapidly emerging, quickly developing and promising technology to generate $3 \mathrm{D}$ tissue constructs with exactly outlined structural geometries utilizing living cells and/or biomaterials as potential bio inks. These bio inks are an important part of bioprinting and generally include biomaterials (such as hydrogels), cells, or cell aggregates, or their combos. Many natural (e.g., 
alginate and gelatin) and artificial (e.g., PCL, PEG, Pluronic) polymers are commonly used as bio inks. Though there are various efforts on advancement of this technology, development of acceptable bio inks that satisfactorily meet bioprinting necessities regarding mechanical, rheological, and biological properties are limited so far. Thus, the advent of latest bio ink materials and engineering of novel bio ink formulations are presently major areas of interest. Additionally, a lot of work is needed in making models and standards to check and judge the properties of various bio ink materials. To the current end, new metrics must be compelled to be developed for evaluating these bio inks and bioprinting processes, that are important to standardize their uses. Additionally, the development of latest computational models is another space of interest to totally analyze the printability and behavior of the bio inks before experimental optimization. Keeping in mind the preceding topics, this review has given top to bottom insights about these bio inks, and it is normal that this might benefit the broad audience in the interesting field of bioprinting and tissue designing in general.

\section{ACKNOWLEDGEMENT}

The authors express their sincere thanks to the Department of Biotechnology, Government of India for the funding from DBT-STAR College grant, under which this review project was conducted. They are also grateful to the Principal and the DBT-STAR Coordinator, Surendranath College, for their support and encouragement in implementing this review project at undergraduate level.

\section{REFERENCES}

Ahlfeld, T., Cidonio, G., Kilian, D., Duin, S., Akkineni, A. R., Dawson, J. I., ... \& Gelinsky, M. (2017). Development of a clay based bioink for 3D cell printing for skeletal application. Biofabrication, 9(3), 034103.

Ali, M., Pr, A. K., Yoo, J. J., Zahran, F., Atala, A., \& Lee, S. J. (2019). A photo-crosslinkable kidney ECM-derived bioink accelerates renal tissue formation. Advanced Healthcare Materials, 8(7), 1800992.

Axpe, E., \& Oyen, M. L. (2016). Applications of alginate-based bioinks in 3D bioprinting. International journal of Molecular Sciences, 17(12), 1976.

Bakirci, E., Toprakhisar, B., Zeybek, M. C., Ince, G. O., \& Koc, B. (2017). Cell sheet based bioink for 3D bioprinting applications. Biofabrication, 9(2), 024105.

Christensen, K., Xu, C., Chai, W., Zhang, Z., Fu, J., \& Huang, Y. (2015). Freeform inkjet printing of cellular structures with bifurcations. Biotechnology and Bioengineering, 112(5), 1047-1055.

Das, S., Pati, F., Choi, Y. J., Rijal, G., Shim, J. H., Kim, S. W., ... \& Ghosh, S. (2015). Bioprintable, cellladen silk fibroin-gelatin hydrogel supporting multilineage differentiation of stem cells for fabrication of three-dimensional tissue constructs. Acta Biomaterialia, 11, 233-246.

DeSimone, E. (2020). Biofabrication Using Recombinant Spider Silk Proteins (Doctoral dissertation, Universität Bayreuth, Bayreuther Graduiertenschule für Mathematik und NaturwissenschaftenBayNAT).

Dzobo, K., Motaung, K. S. C. M., \& Adesida, A. (2019). Recent trends in decellularized extracellular matrix bioinks for 3D printing: an updated review. International Journal of Molecular Sciences, 20(18), 4628.

Fan, R., Piou, M., Darling, E., Cormier, D., Sun, J., \& Wan, J. (2016). Bio-printing cell-laden Matrigel-agarose constructs. Journal of Biomaterials Applications, 31(5), 684-692.

Gillispie, G., Prim, P., Copus, J., Fisher, J., Mikos, A. G., Yoo, J. J., ... \& Lee, S. J. (2020). Assessment methodologies for extrusion-based bioink printability. Biofabrication, 12(2), 022003.

Gu, Q., Tomaskovic-Crook, E., Wallace, G. G., \& Crook, J. M. (2017). 3D bioprinting human induced 
pluripotent stem cell constructs for in situ cell proliferation and successive multilineage differentiation. Advanced Healthcare Materials, 6(17), 1700175.

Gungor-Ozkerim, P. S., Inci, I., Zhang, Y. S., Khademhosseini, A., \& Dokmeci, M. R. (2018). Bioinks for 3D bioprinting: an overview. Biomaterials Science, 6(5), 915-946.

Hölzl, K., Lin, S., Tytgat, L., Van Vlierberghe, S., Gu, L., \& Ovsianikov, A. (2016). Bioink properties before, during and after 3D bioprinting. Biofabrication, 8(3), 032002.

Hong, N., Yang, G. H., Lee, J., \& Kim, G. (2018). 3D bioprinting and its in vivo applications. Journal of Biomedical Materials Research Part B:Applied Biomaterials, 106(1), 444-459.

Hospodiuk, M., Dey, M., Sosnoski, D., \& Ozbolat, I. T. (2017). The bioink: A comprehensive review on bioprintable materials. Biotechnology Advances, 35(2), 217-239.

Huang, Q., Zou, Y., Arno, M. C., Chen, S., Wang, T., Gao, J., ... \& Du, J. (2017). Hydrogel scaffolds for differentiation of adipose-derived stem cells. Chemical Society Reviews, 46(20), 6255-6275.

Jang, J., Park, H. J., Kim, S. W., Kim, H., Park, J. Y., Na, S. J., .. \& Cho, D. W. (2017). 3D printed complex tissue construct using stem cell-laden decellularized extracellular matrix bioinks for cardiac repair. Biomaterials, 112, 264-274.

Kreimendahl, F., Köpf, M., Thiebes, A. L., Duarte Campos, D. F., Blaeser, A., Schmitz-Rode, T., ... \& Fischer, H. (2017). Three-dimensional printing and angiogenesis: tailored agarose-type I collagen blends comprise three-dimensional printability and angiogenesis potential for tissue-engineered substitutes. Tissue Engineering Part C: Methods, 23(10), 604-615.

Kucukgul, C., Ozler, B., Karakas, H. E., Gozuacik, D., \& Koc, B. (2013). 3D hybrid bioprinting of macrovascular structures. Procedia Engineering, 59, 183-192.

López-Marcial, G. R., Zeng, A. Y., Osuna, C., Dennis, J., García, J. M., \& O’Connell, G. D. (2018). Agarose-based hydrogels as suitable bioprinting materials for tissue engineering. ACS Biomaterials Science \& Engineering, 4(10), 3610-3616.

Mandrycky, C., Wang, Z., Kim, K., \& Kim, D. H. (2016). 3D bioprinting for engineering complex tissues. Biotechnology Advances, 34(4), 422-434.

Markstedt, K., Mantas, A., Tournier, I., Martínez Ávila, H., Hagg, D., \& Gatenholm, P. (2015). 3D bioprinting human chondrocytes with nanocellulose-alginate bioink for cartilage tissue engineering applications. Biomacromolecules, 16(5), 1489-1496.

Mirdamadi, E., Muselimyan, N., Koti, P., Asfour, H., \& Sarvazyan, N. (2019). Agarose slurry as a support medium for bioprinting and culturing freestanding cell-laden hydrogel constructs. $3 D$ Printing and Additive Manufacturing, 6(3), 158-164.

Mirski, R., Bekhta, P., \& Dziurka, D. (2019). Relationships between thermoplastic type and properties of polymer-triticale boards. Polymers, 11(11), 1750.

Murphy, S. V., De Coppi, P., \& Atala, A. (2020). Opportunities and challenges of translational 3D bioprinting. Nature Biomedical Engineering, 4(4), 370-380.

Norouzi, M., Nazari, B., \& Miller, D. W. (2016). Injectable hydrogel-based drug delivery systems for local cancer therapy. Drug Discovery Today, 21(11), 1835-1849.

Ono, M. (2017). Resin glycosides from Convolvulaceae plants. Journal of Natural Medicines, 71(4), 591-604.

Piras, C. C., Fernández-Prieto, S., \& De Borggraeve, W. M. (2017). Nanocellulosic materials as bioinks for 3D bioprinting. Biomaterials Science, 5(10), 1988-1992.

Ribeiro, A., Blokzijl, M. M., Levato, R., Visser, C. W., Castilho, M., Hennink, W. E., ... \& Malda, J. (2017). Assessing bioink shape fidelity to aid material development in 3D bioprinting. Biofabrication, 10(1), 014102. 
Sakai, S., Ohi, H., Hotta, T., Kamei, H., \& Taya, M. (2018). Differentiation potential of human adipose stem cells bioprinted with hyaluronic acid/gelatin-based bioink through microextrusion and visible light-initiated crosslinking. Biopolymers, 109(2), e23080.

Shin, M., Galarraga, J. H., Kwon, M. Y., Lee, H., \& Burdick, J. A. (2019). Gallol-derived ECM-mimetic adhesive bioinks exhibiting temporal shear-thinning and stabilization behavior. Acta Biomaterialia, 95, 165-175.

So, W. H., \& Xia, J. (2019). On-Resin Passerini Reaction toward C-Terminal Photocaged Peptides. Organic letters, 22(1), 214-218.

Sun, W., Starly, B., Daly, A. C., Burdick, J. A., Groll, J., Skeldon, G., ... \& Ozbolat, I. T. (2020). The bioprinting roadmap. Biofabrication, 12(2), 022002.

Tumbleston, J. R., Shirvanyants, D., Ermoshkin, N., Janusziewicz, R., Johnson, A. R., Kelly, D., ... \& DeSimone, J. M. (2015). Continuous liquid interface production of 3D objects. Science, 347(6228), 1349-1352.

Vijayavenkataraman, S., Yan, W. C., Lu, W. F., Wang, C. H., \& Fuh, J. Y. H. (2018). 3D bioprinting of tissues and organs for regenerative medicine. Advanced Drug Delivery Reviews, 132, 296-332.

Wang, C., Kelley, S. S., \& Venditti, R. A. (2016). Lignin-based thermoplastic materials. ChemSusChem, 9(8), 770-783.

Wolf, M. T., Daly, K. A., Brennan-Pierce, E. P., Johnson, S. A., Carruthers, C. A., D'Amore, A., ... \& Badylak, S. F. (2012). A hydrogel derived from decellularized dermal extracellular matrix. Biomaterials, 33(29), 7028-7038.

Xiong, S., Zhang, X., Lu, P., Wu, Y., Wang, Q., Sun, H., ... \& Ouyang, H. (2017). Agelatin-sulfonated silk composite scaffold based on $3 \mathrm{D}$ printing technology enhances skin regeneration by stimulating epidermal growth and dermal neovascularization. Scientific reports, 7(1), 1-12.

Xu, C., Zhang, M., Huang, Y., Ogale, A., Fu, J., \& Markwald, R. R. (2014). Study of droplet formation process during drop-on-demand inkjetting of living cell-laden bioink. Langmuir, 30(30), 9130-9138.

Yang, X., Lu, Z., Wu, H., Li, W., Zheng, L., \& Zhao, J. (2018). Collagen-alginate as bioink for threedimensional (3D) cell printing based cartilage tissue engineering. Materials Science and Engineering: C, 83, 195-201.

Yeo, M., Lee, J. S., Chun, W., \& Kim, G. H. (2016). An innovative collagen-based cell-printing method for obtaining human adipose stem cell-laden structures consisting of core-sheath structures for tissue engineering. Biomacromolecules, 17(4), 1365-1375.

Yu, Y., Moncal, K. K., Li, J., Peng, W., Rivero, I., Martin, J. A., \& Ozbolat, I. T. (2016). Three-dimensional bioprinting using self-assembling scalable scaffold-free "tissue strands" as a new bioink. Scientific Reports, 6(1), 1-11.

Zheng, Z., Wu, J., Liu, M., Wang, H., Li, C., Rodriguez, M. J., ... \& Kaplan, D. L. (2018). 3D bioprinting of self-standing silk-based bioink. Advanced Healthcare Materials, 7(6), 1701026. 


\title{
3D Bioprinting: Various Methods and Techniques for Tissue and Organ Regeneration
}

\author{
Sayak Pan, Sritama Chaterjee, Suchandra Chatterjee* \\ Department of Chemistry, Surendranath College, Kolkata, India \\ ${ }^{*}$ Corresponding Author's Email: chatterjeesuchandra01@gmail.com
}

\begin{abstract}
3D bioprinting could be a spearheading innovation permiting manufacture of biomimetic, multiscale, multi-cellular tissues with incredibly confounded tissue microenvironment, complex structure, structure-function relationship, and tissue-explicit integrative and mechanical nonuniformity. 3D bioprinting has acquired energy with the generation of valuable 3D human constructs imitating native tissues/organs. Varied bio-materials like carbohydrates, proteins, and nucleic acids along with nanocomposites are currently a days becoming accustomed to foster biocompatible and transitory frameworks, advancing cell adhesion and multiplication inside the tissues fabricated utilizing 3D printing innovation. In this review, the various fabrication techniques: Pressure-Assisted, MagneticAssisted and Light-Assisted bioprinting, are intended to be outlined, elaborated and compared. Every technique has incontestable individual blessings and drawbacks. With more modern analysis, surely, we would be able to include various strategies together to blend the advantages of every single procedure in not so distant future.
\end{abstract}

\section{Keywords: 3D Bioprinting; Biomaterial; Scaffold; Tissue; Cell; Organ}

\section{INTRODUCTION}

3D printing is a special technique for growing three dimensional solid objects from a digital file. It allows all to form objects layer by layer employing a specific 3D modelling code program. By 1950, the basic idea about 3D printing got developed and since then, this unique technology has brought diverse advancements. Currently this science is utilized not just for non-biological matters but for alive cells and tissues too. At the Wake Forest Institute scientists for the first time used a 3D printer to make an artificial scaffold of a person's bladder in 1999. They then coated that scaffold with cells taken from that patient and with success grew a working organ for the first time. This incident no doubt has set the stage for genuine bioprinting. Current day 3D Bioprinting (Roche et al., 2020) is nothing but a 3D printing with Biomaterials to duplicate elements that can imitate tissues, bones, and blood vessels within the body. Thus, it can be a progressive technology that will finally create a quicker, simpler, and customized remedy for organ transplantation. This relatively effortless approach approves fabrication of geometrically well-defined 3D scaffolds seeded with cells in an amazingly fast, cheap, and highthroughput manner by the researchers. Bio-printed organs and tissues thus created from patient's own cells obviously would reduce the possibility of rejection and may additionally put off the necessity for organ donors. This technology may permit formation of varied tissue structures, like excretory organ tissues, pores, and skin tissues. Even creation of blood vessels and bone location units are also printable leading to a grand advancement in tissue engineering. Some 3D bio-printed liver tissues have already been written by using researchers in Tokyo. 3D bioprinting contributes to indispensable advances of tissue engineering by providing innovative materials acknowledged as Biomaterials. Biomaterials are bioengineered (Bajaj et al., 2014) substance commonly better than the ordinary bodily materials, as properly as tender tissue, and bone. Though the main purpose of 3D bio-printing is to regenerate a whole organ, very little advancement has been seen till date in this regime. Some organs often have problematic and complicated shapes making them more difficult to bio-print. Thus, a bioprinted heart must not entirely meet its structural needs, but would definitely meet the bioorganic process, mechanical load, and electrical sign propagation needs. The extremely personalized and 
highly customized characteristics of $3 D$ printing technology has immense potential in the pharmaceutical industry too. Using 3D bio printing technology, pills with specific dosage can be printed on demand according to individual patients' physical need. Printing tablets with specific shape and structure to control the release rate, printing loose porous pills to reduce swallowing difficulties and printing of transdermal microneedle patches to reduce pain of patients are also possible. 3D bioprinters are currently evolving fast, and they would revolutionize the whole medical business very soon.

\section{LITERATURE REVIEW}

\section{Bio-Printing:}

Bioprinting may be a subcategory of Additive Manufacturing (AM), recognized as Three-Dimensional (3D) printing (Ozbolat, 2015). 3D printing could not be first delineated in 1986 without the help of Charles W. Hull, who for the first time named it as 'stereolithography' and showed that skinny layers of a cloth could be curved with ultraviolet radiation and could be consecutively written in layers to finally fabricate a powerful 3D structure. In 3D bioprinting, layer-by-layer specific positioning of organic materials, biochemicals and residing cells are done with some helpful elements (Roche et al., 2021), that are employed to create those 3D structures. 3D bioprinting relies on three central approaches: Biomimicry, Independent self-assembly and Mini tissue constructing blocks. Either of these ways are judiciously employed by the researchers to fabricate 3D sensible constructs with organic and mechanical properties appropriate for clinical restoration of tissue and organ operation (Biazar et al., 2018). The main motto however is to breed the advanced micro-architecture of Extra Cellular Matrix (ECM). Over the past decade, a variety of bioprinting applied sciences have been developed (Charbe, McCarron \& Tambuwala, 2017) and tailored to manufacture tissues or organs. The foremost popular bioprinting ways is the pressure-assisted one. Other methodologies like piezo-, thermal-, laser-, acoustic, and microfluid-driven bioprinting are less fashionable being highly expensive. The first principle of $3 \mathrm{D}$ printing is generally followed for generating medical specialty elements that are biocompatible (Yang et al., 2017) and are called bio-inks containing bioactive components like live cells in them in addition. Production of novel bio-inks is really an interesting topic of current day research in medical specialty engineering. Inkjet bioprinters through generation of little ink droplets on a substrate can manufacture notably high-resolution patterns (Xiang Gu et al., 2016) using liquid bio inks. Inkjet, extrusion, and laser-assisted bioprinting are most popular methodologies used in the 3D bioprinting regime. Different other techniques like Freeform Reversible Embedding of Suspended Hydrogels (FRESH) (Hinton et al., 2015) had been tested so far for tissue regeneration. One amongst the brand-new advances in 3D printing field that can dramatically improve the printing time. is "Continuous Liquid Interface Production" developed by DeSimone and his group. Although this technology is no longer used in bioprinting (Ning \& Chen, 2017) but can be fruitful in the long run for printing difficult tissue architectures. In this review, we are going to discuss about some popular techniques of 3D bioprinting and compare their effectiveness

\section{Bioprinting approach:}

There are three central approaches of 3D bioprinting ( $\mathrm{Gu}$ et al., 2015). They are biomimicry, autonomous self-assembly and mini tissue building blocks.

Biomimicry: The primary approach of bioprinting is named biomimicry (du Plessis \& Broeckhoven, 2019). The main aim of this approach is to form fabricated structures exactly mimicking the natural structures that are normally found within the tissues and organs in a body i.e., identical reproduction of the cellular and extracellular parts of a tissue or organ. Material analysis, cell-culture methods, and nanotechnology (Di Marzio et al., 2020) are effectively used to generate all these bio-engineered products. For successful Biomimicry, exact replication of biological tissues in microscale is important.

Autonomous self-assembly: It depends on the cell type being the primary driver of histogenesis, leading to composition, localization, and structural properties of the constructed tissue. It often requires detailed data about embryonic tissue genesis and organogenesis to ultimately drive the embryonic mechanisms of bio-printed tissues. 
Mini tissue building block: In this technique, small functional units of tissues and organs known as mini tissues, are initially formed. These mini tissues represent the smallest possible structural and purposeful units of the organs, just like the kidney neuron that are later fabricated either via autonomous self-assembly or biomimicry. Bioprinting firstly transforms cluster of mini tissues into macro-tissues (Murata, Arai \& Nakayama, 2020) which is next followed by autonomous self-assembly to make desired functional structures.

\section{Imaging and digital design:}

For tissue engineers medical imaging technology is a valuable tool that is used to produce data on 3D structure and its functionality at cellular, tissue, organ, and organism levels. This imaging technology mainly consists of two most popular non-invasive imaging techniques; Computerized Tomography (CT) and Magnetic Resonance Imaging (MRI). Computer-Aided Manufacturing (CAD-CAM) and mathematical modelling are also used to gather advanced tomographic and architectural information about various tissues and organs. The $C T$ imaging required for various medicinal and interventional procedures essentially require absorption of X-rays by the target tissues and organs (Figure 1).
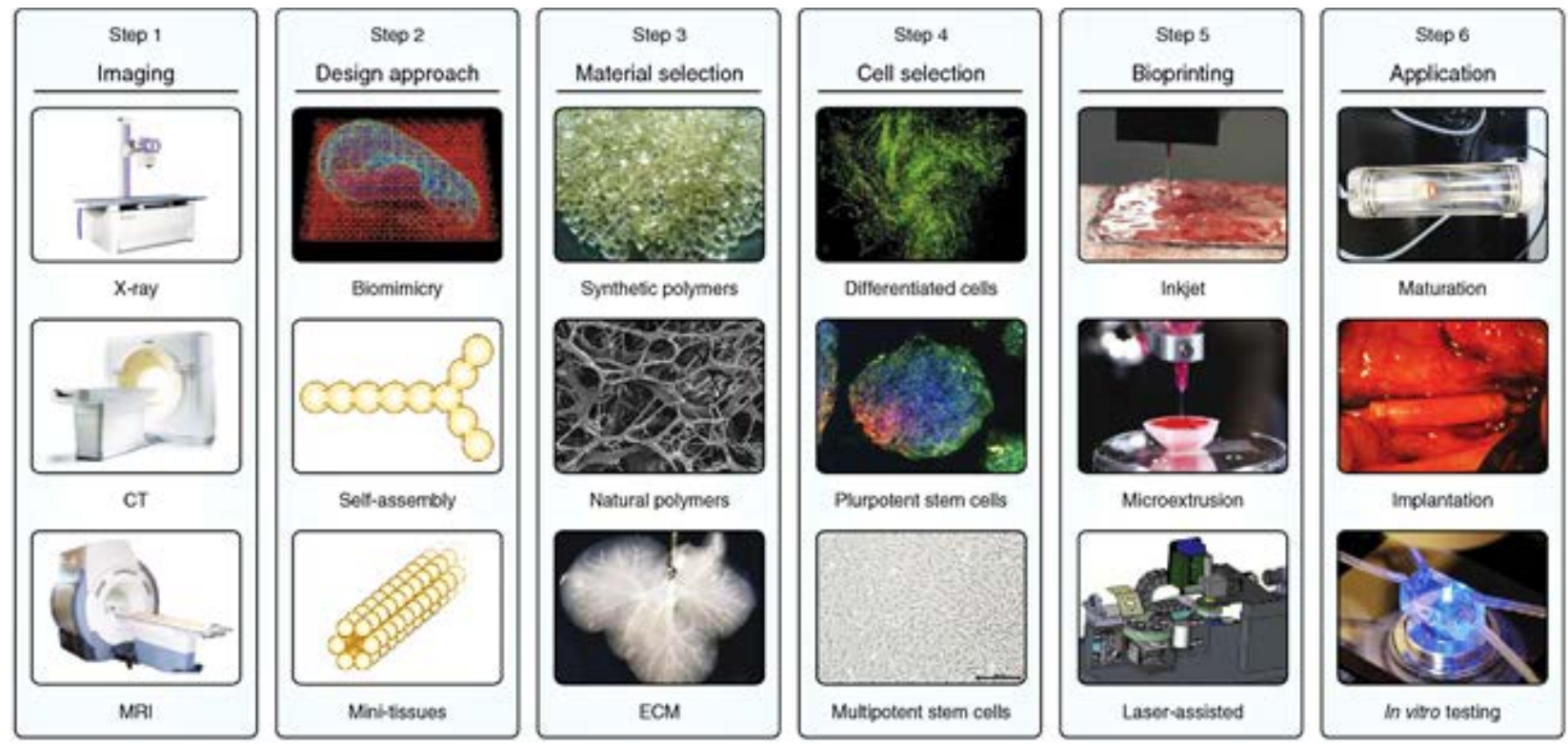

Figure 1: Various Imaging Technique (Xing et al., 2020)

These imaging modalities using $X$ ray beam can generate closely spaced axial slices of tissue design, which after surface rendering and stereolithographic editing, can totally describe whole volume of the target tissue. MRI using Nuclear Magnetic Resonance (NMR) (Kérourédan et al., 2018), can also give high contrast spatial resolution in case of soft tissues without exposure to harmful radiations. The colour contrast of biological structures in this process however can be raised with intelligently utilizing some contrast reagents. In CT scans (Oliveira et al., 2019) barium or iodine are used whereas, iron oxide, gadolinium or metalloproteins are used for MRI scans for the contrast enhancement. These contrast agents can attenuate $X$-rays or enhance magnetic resonance signals effectively highlighting the structures like blood vessels, that otherwise would be tough to delineate from their surroundings. Once the raw image is obtained using these techniques, 2D cross-sectional pictures and 3D anatomical representations are stepwise created for detailed analysis of the structure. These ultimate 3D anatomical representations can generate actual views of organ anatomy religiously holding the imagevoxel info that may be used for volume rendering, volumetric representation, and 3D image representation. Reconstructed images or models thus produced can be viewed from multiple angles to ultimately generate a correct copy of the imaged organ or tissue that is exactly equivalent to the direct copy of a patients' own organ. Computer-based models can also entirely or partly contribute to these anatomical structural design analyses, and simulation. Computer modelling can in addition also assist in predicting mechanical and biochemical properties of the fabricated tissue constructs (Daly et al., 2017). 


\section{DISCUSSION}

\section{Techniques:}

The main technologies used for deposition and patterning of biological materials are inkjet, micro extrusion, laser-assisted printing magnetic-assisted printing and piezo-assisted printing. Unique features of those technologies ought to be thought about considering the foremost crucial factors in 3D bioprinting; surface resolution, cell viability and the biological materials used for printing. Let us explore those important printing techniques as shown in Figure 2.

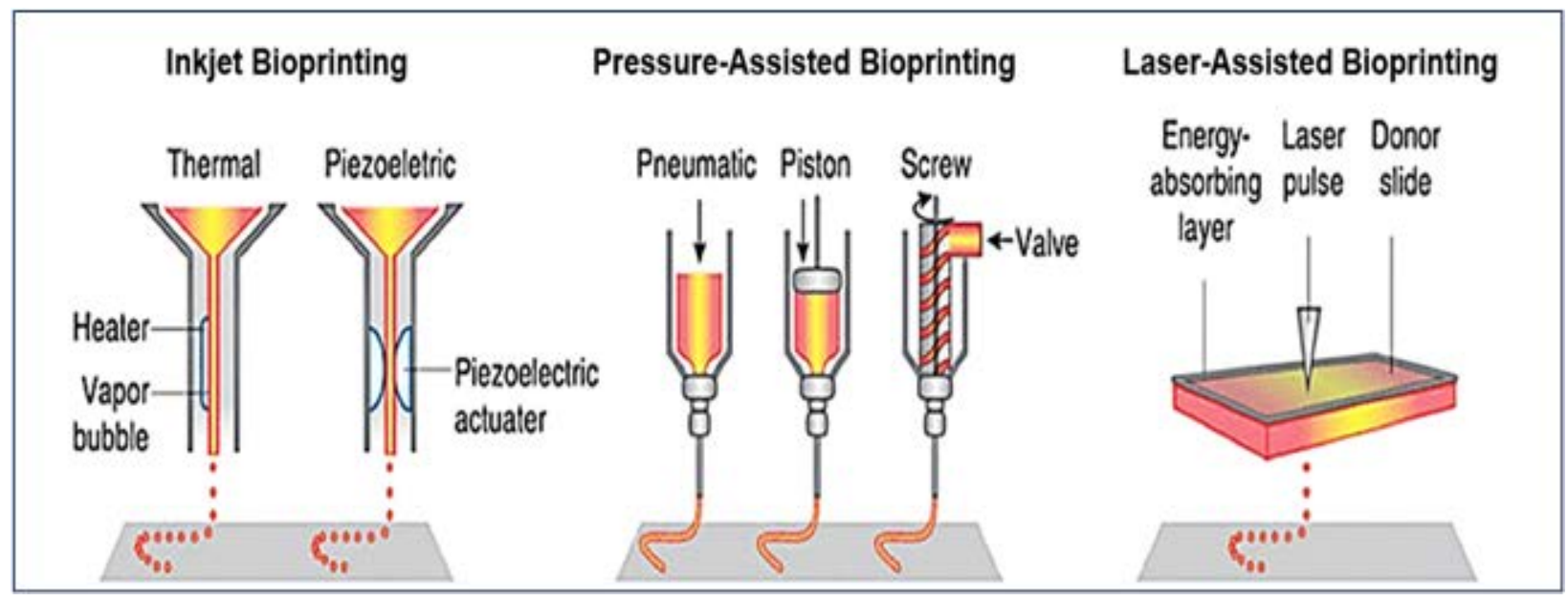

Figure 2: Different Techniques of Bioprinting (Maina et al., 2018)

(a) Pressure-assisted bioprinting: This (PAB) method is primarily based on extrusion technique to construct desired 3D patterns and structures (Cui et al., 2020). Biomaterials in the form of solutions, pastes, or dispersions are generally used for printing here that are intelligently extruded by efficiently

controlling the gas pressure. Once layer-by-layer application is done properly, complete 3D patterns and constructs are eventually shaped almost accurately. Direct incorporation of cells, their undiversified distribution, higher cell density and confirmed retention of activity are the major benefits of this technique however resulting in a lower speed and determination. Wide acceptable range of printable biomaterials and cheap instrumentation are also some of the major benefits of this process. Several researchers have merely changed the standard industrial 3D printers to print biomaterials or developed their own printing machines to scale back the costing. Those printers are commonly equipped with a lot of cartridges filled with chosen bio-inks (Askari et al., 2021). that enable dispensing of various combos of cells and biomaterials simultaneously. Application of pressure facilitates here the ejection of fabric through a needle or nozzle. Various dispensing systems of this PAB method are shown in Figure 2. Direct incorporation cum homogenous distribution of cells and confirmed retention of their activities are the major benefits of this technology. Pressure bioprinters (Moncal et al., 2019) are accustomed to fabricating multiple tissue varieties like artery valves, branched tube-shaped tree like structure and in vitro pharmacokinetic models for tumours. For complicated structures the fabrication time is relatively higher, and the structure of tissues constructed by this process generally vary from normal to micro range.

(b) Thermal-assisted bioprinting: This is the oldest bioprinting technique ever reported. In this method a workplace inkjet printer is generally modified to permit the dispensing of cells and biomaterials (Figure 2). In droplet-on-demand (Solis et al., 2019) (DOD) technique, electrical energy is employed to produce heat that can vaporize the fabric ultimately forming a drop (Figure 2). This vaporization is the reason for the unforeseen enlargement of the fabric in little capillary channel, prompting formation and ejection of drops of fabric. The amount of heat applied here, influences the cell viability throughout and post bioprinting. The resolution of this method is mostly dependent on the amount of bio-ink ejected from each nozzle varying from $\mathrm{pm}$ to $\mathrm{nm}$ with a resolution as low as $100 \mu \mathrm{m}$. 
(c) Inkjet bioprinting: This method initially used a commercial printer only to spray cells. Inkjet bioprinters (Figure 3), often known as droplet bioprinters, generally use thermal energy (Cui et al., 2012) or acoustic force for ejecting liquid drops onto a substrate aiming its layer-by-layer construction.

Thermal inkjet bioprinting normally generates bio-ink droplets by electrical heating of the print head to strain the cells in liquid form and those liquid drops come out from the nozzle with application of pressure. Accurate deposition of bio-inks manufactured from cells, scaffold materials and growth factors are possible by this process. It is the oldest biological printing technique adopting noncontact printing technology using bio inks (cells or biological materials) to comprehend fabrication of organs with the help of digital models. Thermal inkjet (Li et al., 2020) printing technology primarily uses its components to spray droplets. These components are employed to heat the ink quickly, so that the ink within the cavity can be safely vaporized into bubbles. The pressure generated from chemical action can transform those bubbles into a driblet, that can be easily squeezed out of the extrusion nozzle.

Electricity inkjet printing technique primarily

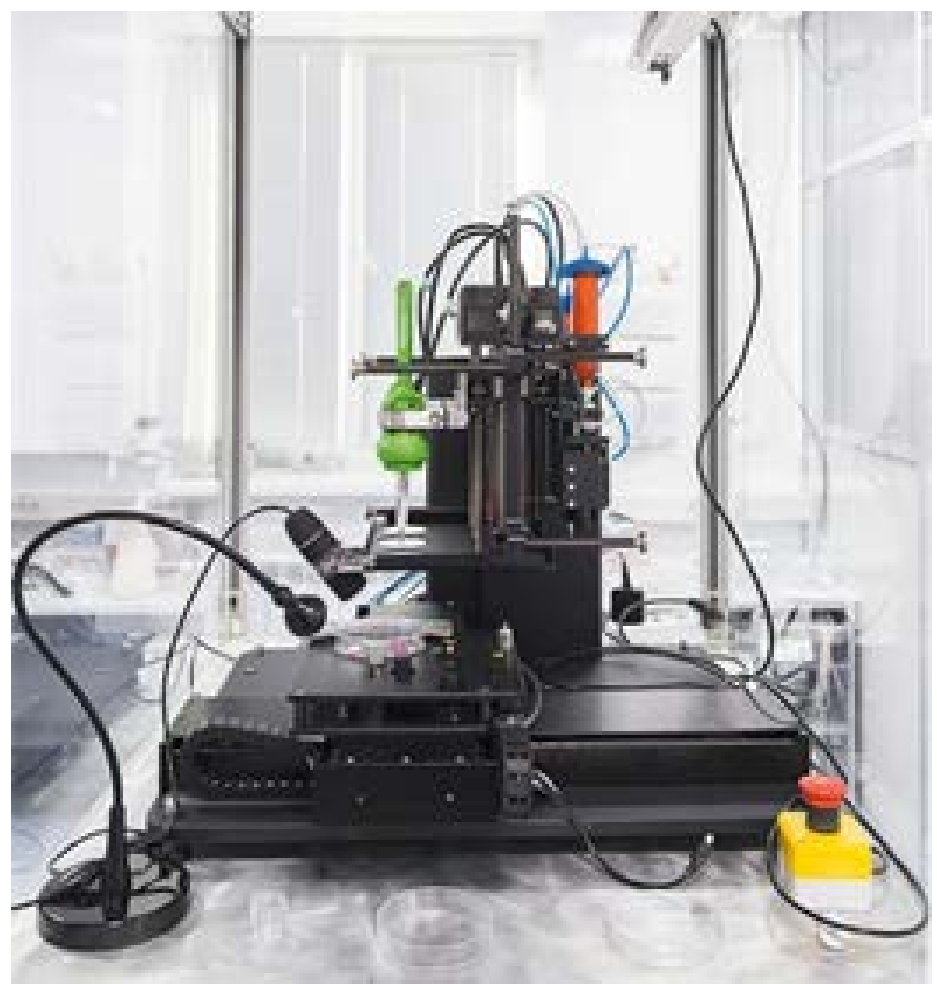

Figure 3: A typical Inject Bioprinter uses electricity for spraying droplets (Cui et al., 2012) for the first-time used this technology for repairing articular gristle of physical structure, that was evidenced to be a potential guide for the tissues to regenerate expeditiously. Weiss et al. later used multi-nozzles for manufacturing composite structures. Utilizing this method, a range of defective growth factors, like clotting factor, coagulase etc. could be written exactly into a mice cell. They also showed feasibility of unaltered printing that due to its complexity was found inappropriate for sensible use. This inkjet printing technology (Angelopoulos et al., 2020) used to be preferred earlier due to its comparatively matured nature with its multiple nozzles, high speed to synchronously print cells, non-contact approach and capability of building heterogeneous tissues and organs as well. Compared to other alternative methods, this inkjet printing technique offers quick fabrication speed but with comparatively low cell densities. The methodology can be classified into three groups: Continuous inkjet bioprinting, Electro-hydrodynamic jet bioprinting, and Drop-on-demand inkjet bioprinting.

(d) Piezo-assisted bioprinting: When piezoelectric materials are stirred up electrically, they ordinarily do get deformed in size. In this technique ejection of very little volumes of bio ink even in driblet is possible just like thermal-assisted bioprinting process. Using this method, it is feasible to dispense droplets with volume $\mathrm{pl}$ to $\mathrm{nl}$ and hence (Ahn et al., 2016) can safely be employed for fabricating microarrays and deposition of interstitial tissues and retinal neural cells not affecting the cell viability.

(e) Magnetic-assisted bioprinting: Permanent magnets or temporary electromagnets are used in this process to produce magnetic field that can be extensively used for patterning of the cells in controlled arrays or locations. Pre-labelling of cells with magnetic nanoparticles or magnetic fluids (Gupta et al., 2020) is essential here for management of cells prior application of magnetic field. Even single-cell patterning is also possible by this method. As these technologies mature, it would not be impossible to mix this magnetic field to arrange cells into definite patterns during any bioprinting method or even post bioprinting. 
(f) Laser-assisted bioprinting: In general light-assisted bioprinting are methods are termed as stereolithography (SLA). There are two distinctive varieties of SLA techniques: Optical Laser and Digital Lightweight Process (DLP). To compare, while optical laser can induce selective point by point cross-linking of a light-sensitive material, the DLP system utilizing a dynamic mirror array can selectively project light onto larger areas to cross-link all points assigned for every layer. Many other alternative light-based techniques were explored later (Hakobyan et al., 2020). In such laser assisted processes, a pulsed light beam is generally targeted on a glass surface coated with an adsorbed layer that vaporizes to induce driblet formation and deposition of those droplets layer-by-layer with a predesigned pattern till the desired 3D construct is formed. This nozzle-free technique typically avoids shear stress on cells commonly observed in other available techniques. Two-Photon Polymerisation (2PP) is another such technique permitting high-accuracy cross-linking (Sorkio et al., 2018) by selective irradiation and desired cross-linking of the voxels. Because of the high-resolution structures attainable through 2PP, the build time is comparatively long, and the sizes of the created constructs commonly range between hundreds of microns to a couple of millimetres.

(g) Microfluidics bioprinting: Microfluidics bioprinting (as shown in Figure 4) is a comparatively old methodology initiated in the 1950's widely adopted and applied wherever cells and biomaterials are cultivated in extremely outlined and controlled conditions. The chance to regulate fluid flow accurately by making certain laminar regimes allow the researchers to analyse cellular environments much accurately and effectively reducing the number of essential ingredients. Most modern bioprinters use custom-made microfluidic chip points as dispensing heads using which, it can style up new types of bioprinting systems that enable proper dispensing of low consistency materials combined with cells (Figure 4). One of the major benefits of this technique is that it is supported by a well-established
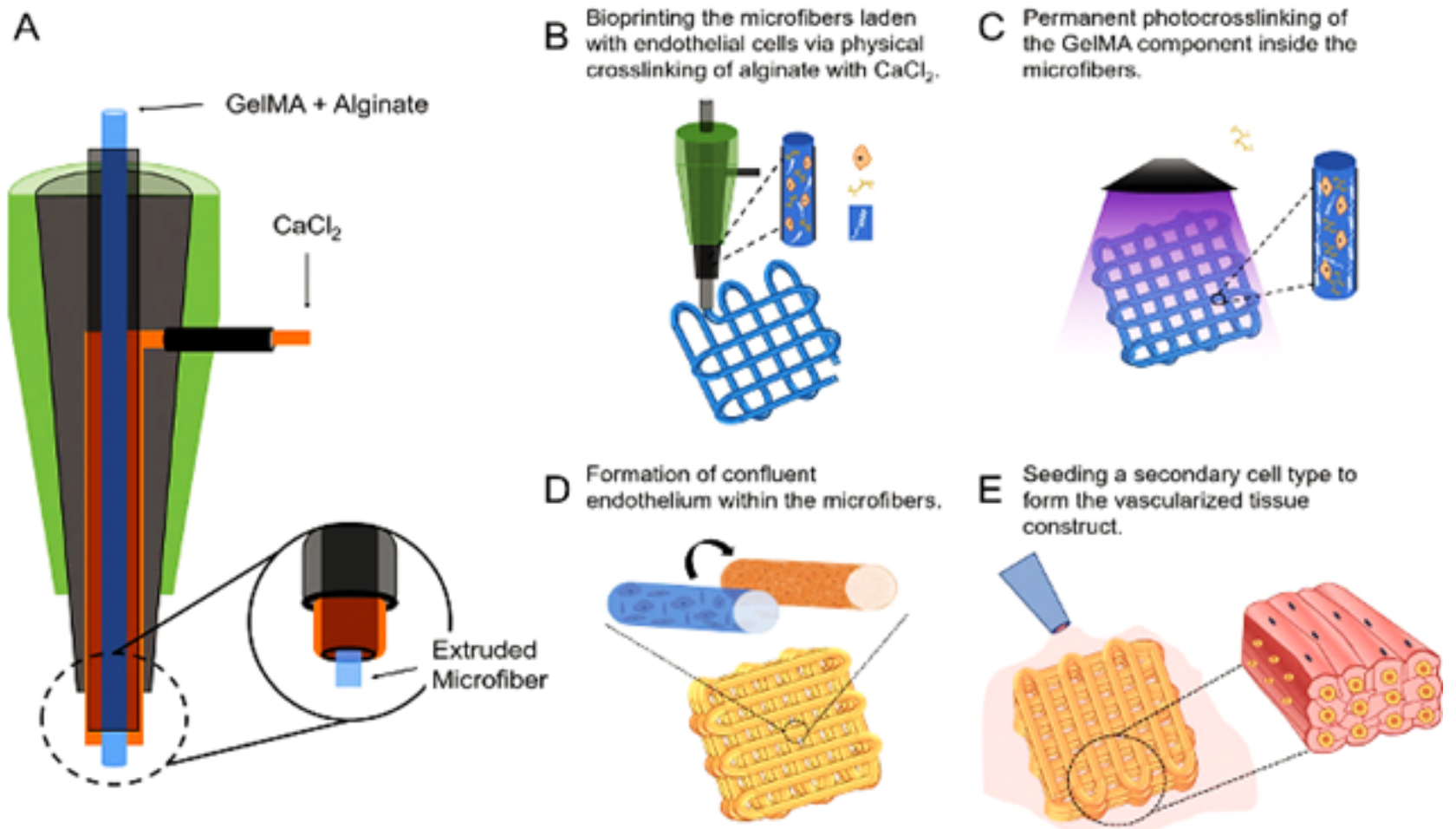

Figure 4: The Microfluidic Bioprinting Strategy for Generating Vascularized Tissue Constructs (Zhang, Pi \& van Genderen, 2017)

methodology, thus making development of various heads much easier (Richard, Neild \& Cadarso, 2020). Some commercial bioprinters have already adopted this technology. Microfluidics can correctly manage the fluids carrying biomolecules, cells, organisms, or chemical agents and are ideal for performing a mess of tasks and are extremely useful in integrating diverse types of 3D bioprinting nozzles. 


\section{CONCLUSION}

3D bioprinting innovations have plenty of degrees for added improvement. Organ printing for clinical applications is yet a work in progress. A typical obstacle of bioprinting strategy is its low printing speed for producing complex scale-up structures. While a few difficulties still needing to be tended to in carrying out full clinical interpretation of bioprinting, there have been some extremely encouraging advancements in the previous few years. Development of skin, bone, ligament, vessels, organoids, and so on using 3D bioprinting advances have shown a great deal of potential, and the blend of bioprinting and drug science likewise have brought up another novel heading for future exploration. Numerous private companies like CELLINK, Organovo, TeVido, Digilab, Cyfuse are currently commercializing this innovation. Along these lines, in this background, we should say that 3DBioprinting definitely has an exceptionally promising future.

\section{ACKNOWLEDGEMENT}

The authors express their sincere thanks to the Department of Biotechnology, Government of India for the funding from DBT-STAR College grant, under which this review project was conducted. They are also grateful to the Principal and the DBT-STAR Coordinator, Surendranath College, for their support and encouragement in implementing this review project at the undergraduate level.

\section{REFERENCES}

Ahn, S. H., Lee, J., Park, S. A., \& Kim, W. D. (2016). Three-dimensional bio-printing equipment technologies for tissue engineering and regenerative medicine. Tissue Engineering and Regenerative Medicine, 13(6), 663-676.

Angelopoulos, I., Allenby, M. C., Lim, M., \& Zamorano, M. (2020). Engineering inkjet bioprinting processes toward translational therapies. Biotechnology and Bioengineering, 117(1), 272-284.

Askari, M., Naniz, M. A., Kouhi, M., Saberi, A., Zolfagharian, A., \& Bodaghi, M. (2021). Recent progress in extrusion 3D bioprinting of hydrogel biomaterials for tissue regeneration: a comprehensive review with focus on advanced fabrication techniques. Biomaterials Science, 9(3), 535-573.

Bajaj, P., Schweller, R. M., Khademhosseini, A., West, J. L., \& Bashir, R. (2014). 3D biofabrication strategies for tissue engineering and regenerative medicine. Annual Review of Biomedical Engineering, 16, 247-276.

Biazar, E., Najafi S, M., Heidari K, S., Yazdankhah, M., Rafiei, A., \& Biazar, D. (2018). 3D bio-printing technology for body tissues and organs regeneration. Journal of Medical Engineering \& Technology, 42(3), 187-202.

Charbe, N., McCarron, P. A., \& Tambuwala, M. M. (2017). Three-dimensional bio-printing: A new frontier in oncology research. World Journal of Clinical Oncology, 8(1), 21.

Cui, X., Boland, T., DD'Lima, D., \& K Lotz, M. (2012). Thermal inkjet printing in tissue engineering and regenerative medicine. Recent Patents on Drug Delivery \& Formulation, 6(2), 149-155.

Cui, X., Li, J., Hartanto, Y., Durham, M., Tang, J., Zhang, H., ... \& Woodfield, T. (2020). Advances in Extrusion 3D Bioprinting: A Focus on Multicomponent Hydrogel-Based Bioinks. Advanced Healthcare Materials, 9(15), 1901648.

Daly, A. C., Freeman, F. E., Gonzalez-Fernandez, T., Critchley, S. E., Nulty, J., \& Kelly, D. J. (2017). 3D bioprinting for cartilage and osteochondral tissue engineering. Advanced Healthcare Materials, 6(22), 1700298.

Di Marzio, N., Eglin, D., Serra, T., \& Moroni, L. (2020). Bio-Fabrication: Convergence of 3D bioprinting and nano-biomaterials in tissue engineering and regenerative medicine. Frontiers in Bioengineering and Biotechnology, 8, 326. 
du Plessis, A., \& Broeckhoven, C. (2019). Looking deep into nature: A review of micro-computed tomography in biomimicry. Acta Biomaterialia, 85, 27-40.

Gu, Q., Hao, J., Lu, Y., Wang, L., Wallace, G. G., \& Zhou, Q. (2015). Three-dimensional bio-printing. Science China Life Sciences, 58(5), 411-419.

Gupta, T., Aithal, S., Mishriki, S., Sahu, R. P., Geng, F., \& Puri, I. K. (2020). Label-Free Magnetic-FieldAssisted Assembly of Layer-on-Layer Cellular Structures. ACS Biomaterials Science \& Engineering, 6(7), 4294-4303.

Hakobyan, D., Kerouredan, O., Remy, M., Dusserre, N., Medina, C., Devillard, R., ... \& Oliveira, H. (2020). Laser-assisted bioprinting for bone repair. In 3D Bioprinting (pp. 135-144). Humana, New York, NY.

Hinton, T. J., Jallerat, Q., Palchesko, R. N., Park, J. H., Grodzicki, M. S., Shue, H. J., ... \& Feinberg, A. W. (2015). Three-dimensional printing of complex biological structures by freeform reversible embedding of suspended hydrogels. Science Advances, 1(9), e1500758.

Kérourédan, O., Ribot, E. J., Fricain, J. C., Devillard, R., \& Miraux, S. (2018). Magnetic Resonance Imaging for tracking cellular patterns obtained by Laser-Assisted Bioprinting. Scientific Reports, 8(1), 1-10.

Li, X., Liu, B., Pei, B., Chen, J., Zhou, D., Peng, J., ... \& Xu, T. (2020). Inkjet bioprinting of biomaterials. Chemical Reviews, 120(19), 10793-10833.

Maina, R. M., Barahona, M. J., Finotti, M., Lysyy, T., Geibel, P., D’Amico, F., ... \& Geibel, J. P. (2018). Generating vascular conduits: from tissue engineering to three-dimensional bioprinting. Innovative Surgical Sciences, 3(3), 203-213.

Moncal, K. K., Ozbolat, V., Datta, P., Heo, D. N., \& Ozbolat, I. T. (2019). Thermally-controlled extrusionbased bioprinting of collagen. Journal of Materials Science: Materials in Medicine, 30(5), 1-14.

Murata, D., Arai, K., \& Nakayama, K. (2020). Scaffold-Free Bio-3D Printing Using Spheroids as "Bio-Inks" for Tissue (Re-) Construction and Drug Response Tests. Advanced Healthcare Materials, 9(15), 1901831.

Ning, L., \& Chen, X. (2017). A brief review of extrusion-based tissue scaffold bio-printing. Biotechnology Journal, 12(8), 1600671.

Oliveira, E. P., Malysz-Cymborska, I., Golubczyk, D., Kalkowski, L., Kwiatkowska, J., Reis, R. L., ... \& Walczak, P. (2019). Advances in bioinks and in vivo imaging of biomaterials for CNS applications. Acta Biomaterialia, 95, 60-72.

Ozbolat, I. T. (2015). Bioprinting scale-up tissue and organ constructs for transplantation. Trends in Biotechnology, 33(7), 395-400.

Richard, C., Neild, A., \& Cadarso, V. J. (2020). The emerging role of microfluidics in multi-material 3D bioprinting. Lab on a Chip, 20(12), 2044-2056.

Roche, C. D., Brereton, R. J., Ashton, A. W., Jackson, C., \& Gentile, C. (2020). Current challenges in three-dimensional bioprinting heart tissues for cardiac surgery. European Journal of Cardio-Thoracic Surgery, 58(3), 500-510.

Roche, C. D., Sharma, P., Ashton, A. W., Jackson, C., Xue, M., \& Gentile, C. (2021). Printability, Durability, Contractility and Vascular Network Formation in 3D Bioprinted Cardiac Endothelial Cells Using Alginate-Gelatin Hydrogels. Frontiers in Bioengineering and Biotechnology, 9, 110.

Solis, L. H., Ayala, Y., Portillo, S., Varela-Ramirez, A., Aguilera, R., \& Boland, T. (2019). Thermal inkjet bioprinting triggers the activation of the VEGF pathway in human microvascular endothelial cells in vitro. Biofabrication, 11(4), 045005. 
Sorkio, A., Koch, L., Koivusalo, L., Deiwick, A., Miettinen, S., Chichkov, B., \& Skottman, H. (2018). Human stem cell based corneal tissue mimicking structures using laser-assisted 3D bioprinting and functional bioinks. Biomaterials, 171, 57-71.

Xiang Gu, G., Su, I., Sharma, S., Voros, J. L., Qin, Z., \& Buehler, M. J. (2016). Three-dimensionalprinting of bio-inspired composites. Journal of Biomechanical Engineering, 138(2).

Xing, F., Xiang, Z., Rommens, P. M., \& Ritz, U. (2020). 3D bioprinting for vascularized tissue-engineered bone fabrication. Materials, 13(10), 2278.

Yang, R., Chen, Y., Ma, C., Wang, H., \& Wang, S. (2017). Research progress on the technique and materials for three-dimensional bio-printing. Sheng wu yi xue gong cheng xue za zhi= Journal of Biomedical Engineering= Shengwu Yixue Gongchengxue Zazhi, 34(2), 320-324.

Zhang, Y. S., Pi, Q., \& van Genderen, A. M. (2017). Microfluidic bioprinting for engineering vascularized tissues and organoids. Journal of Visualized Experiments: JoVE, (126). 


\title{
An Overview on The Threats and Conservation Strategies of Royal Bengal Tiger (Panthera tigris tigris) in Sundarban, India
}

\author{
Aniket Chatterjee, Disha Chatterjee, Biswajit Santra, Adity Sarbajna* \\ Department of Zoology, Surendranath College, Kolkata, India \\ ${ }^{*}$ Corresponding Author's Email: aditymukhopadhyay@gmail.com
}

\begin{abstract}
The largest contiguous Lagoon Wetland in the world is sundarban. The magnificent endangered species among the animals on land is Royal Bengal Tiger. As per Red data book the species is highly threatened. The effect of ecological degradation of Sundarban in the present status of Panthera tigris tigris is clearly reflected by its number in its natural number. It is difficult to conserve tigers embedded within densely populated human dominated landscapes and it causes conflict between human and tiger. The main causes of this Conflict is the livestock grazing and human intrusion into tiger habitat and also poor husbandry So, protection of tiger can be performed by providing them adequate grazing area, abundance of prey, and proper monitoring of their lives. Wide population size and adjacent habitat of sundarban is apparently penetrable to tigers so, the sundarban is very much valuable for tiger conservation. This paper examines the habitat of tiger and its threats and conservation through the Project Tiger in the Sundarban Biosphere Reserve.
\end{abstract}

Keywords: - Royal Bengal Tiger; Panthera Tigris; Sundarban; Conservation

\section{INTRODUCTION}

Sundarban Biosphere Reserve is the world's largest abutting rainforest ecosystem. The estuary is formed due to the confluence of three significant rivers, the Ganges, Meghna \& Brahmaputra which intersect at the valley of the Bay of Bengal. An total area of $10,248 \mathrm{sq} \mathrm{km}$ is covered by Sundarban of which $41.5 \%$ is in India (Fig:1). The Sundarbans houses over 1,500 species of fauna as well as 84 different mangroves and mangrove associated vegetation (Raha \& Saha, 2004) and is the only mangrove forest in the world inhabited by tigers.

Sundarban Tiger Reserve covers an area of $2,548.89 \mathrm{sq} \mathrm{Km}$ and was established in 1973. It is situated a small south of the Tropic of Cancer between $21^{\circ} 31^{\prime}$ to $22^{\circ} 53^{\prime} \mathrm{N}$ and $88^{\circ} 37$ to $89^{\circ} 09^{\prime} \mathrm{E}$. It possess three delegate zones: core zone: $1,330.12 \mathrm{~km} 2$; primitive zone: $124.40 \mathrm{~km} 2$; subsidiary wilderness zone: $241.07 \mathrm{~km} 2$, and buffer zone $(1,255 \mathrm{~km} 2)$ (Chowdhury et al., 2016).

Tigers of Sundarbans are discovered throughout the length and width of the forests and are classified as 'Endangered' in The IUCN Red List of Threatened Species ${ }^{\mathrm{TM}}$. Though they are infamous as maneaters but are popular as long-range swimmers, efficient climbers having extraordinary migratory and eco-adaptive behaviors (Mallick, 2013).

When wild Tiger (Panthera tigris tigris) dominated area merges with human dominated landscapes, they cause a threat by preying on livestock and less commonly on humans. Human - Wildlife conflict (HWC) causes severe harm and it is a serious global problem .It is very harmful for so many endangered species for their living, apace with the loss of human life and their property (Chowdhury et al., 2016).Fuel wood dependency in area, forest non-timber product collection, illegal grazing etc also is the key cause of conflicts (Kumar, 2020). According to Kumar, 2020, movement in human dominated areas and interaction with human beings has enhanced tiger's skills along with cognition for survival without any fear and current pandemic and subsequent lock down has resulted in less anthropogenic 
stress in the natural habitat thereby enhancing fear less movement of wild animals and increasing chances of $\mathrm{HWC}$ in future.

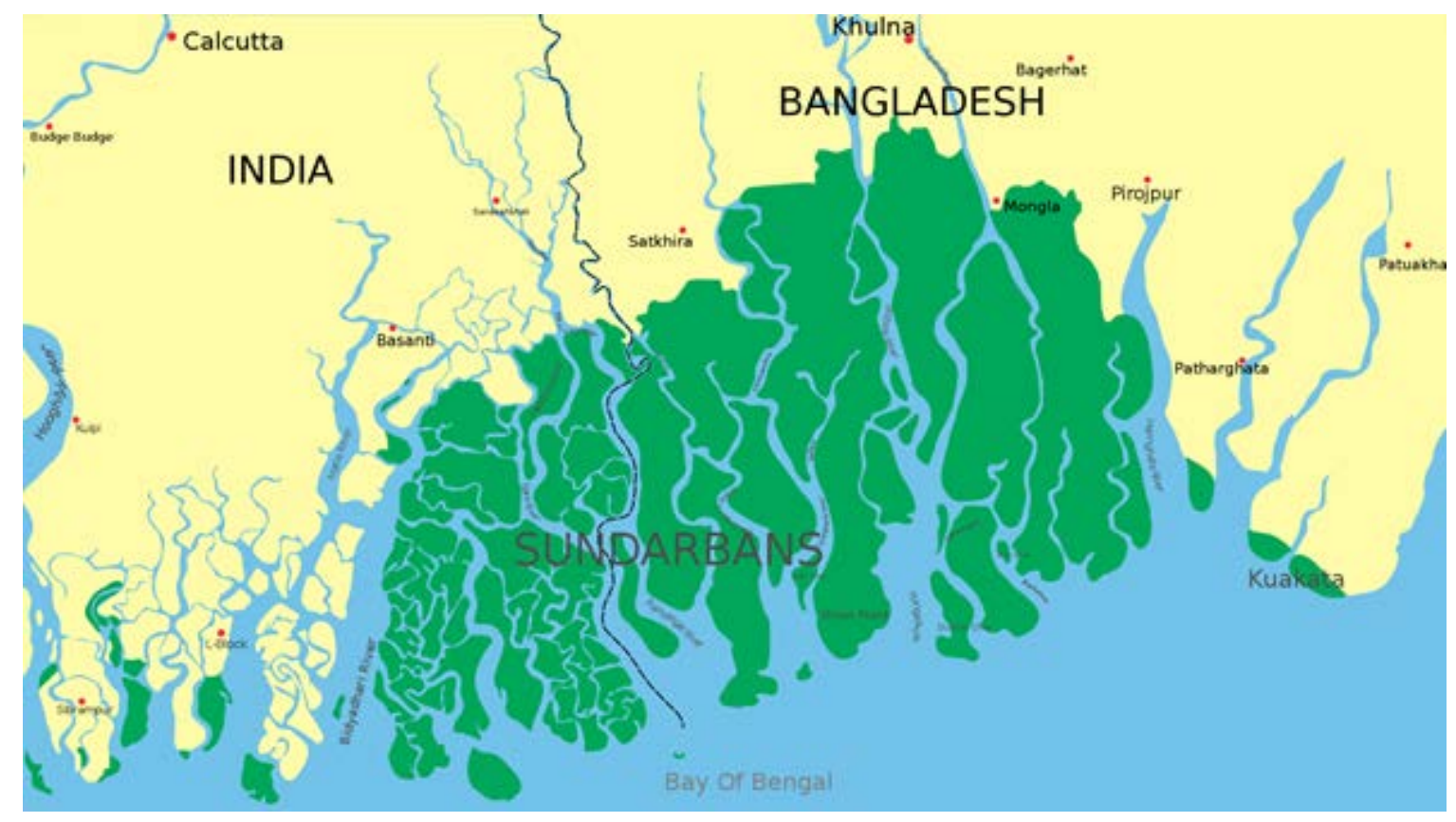

Figure 1: Sundarban Delta

\section{LITERATURE REVIEW}

\section{The Sundarban Biosphere Reserve}

In West Bengal , Sundarban Tiger Reserve (2584.89 sq km) is the only mangrove tiger land and it is a part of the World's largest estuarine wetland spread over Bangladesh and India (Mallick, 2012). The area provides the subcontinent's largest population of the critically endangered species of Royal Bengal Tigers (Panthera tigris tigris), which are well known for their long distance swimming and extra ordinary migrating behavior from one island to another and are also popular for their reputation as man- eaters (Chowdhury et al., 2016).

\section{In-situ conservation}

In Sundarbans there are about 550 individuals of Royal Bengal Tiger are present respectively and nearly all reports that exist on wildlife and tigers in the Sundarbans mention the large number of tiger attacks and discuss the possible root causes the impact of tiger attacks of in- situ in Bangladesh. (Azad, Hashem \& Hossain, 2005). Sundarbans is a unique, relatively unfragmented ecosystem and has significant socio-economical and ecological values, thus receiving considerable conservation attention from government and implications on society, culture, sustainability and the biodiversity of Bangladesh. (Ashraf, 2010). Occasionally the concurrence of both human-beings and tigers in in-situ areas creates the context in which tigers are attacked and killed as a measure of retribution, Considering the economic sustainability and livelihood of the local people, the conservation of tigers has become a serious issue in the Sundarbans .(Dey, Debnath \& Sikdar, 2006).

\section{Habitat of Royal Bengal Tiger in Sundarban}

The tiger population inhabiting the Sundarbans are very peculiar with reference to their adaptations to the mangrove forests and one of the greatest diverse tiger habitat in the world (Naha et al., 2016).Tigers of Sundarbans are relatively occupy large habitats, they are terrestrial and the size of the area usually depends on the density of their prey (Bhattarai, 2009). Tiger occurrence must be varying from area to area based on its own likings; they do not have any specific area for lead their life the mangrove (Bhattacharya, 2011). Naha et al., 2016, identified five habitat classes of tigers in 
Sundarban based on ground cover (Figure1) namely, Water/Channels, Phoenix dominated, Ceriops dominated, Barren dry areas and Avicennia-Sonneratia habitats.

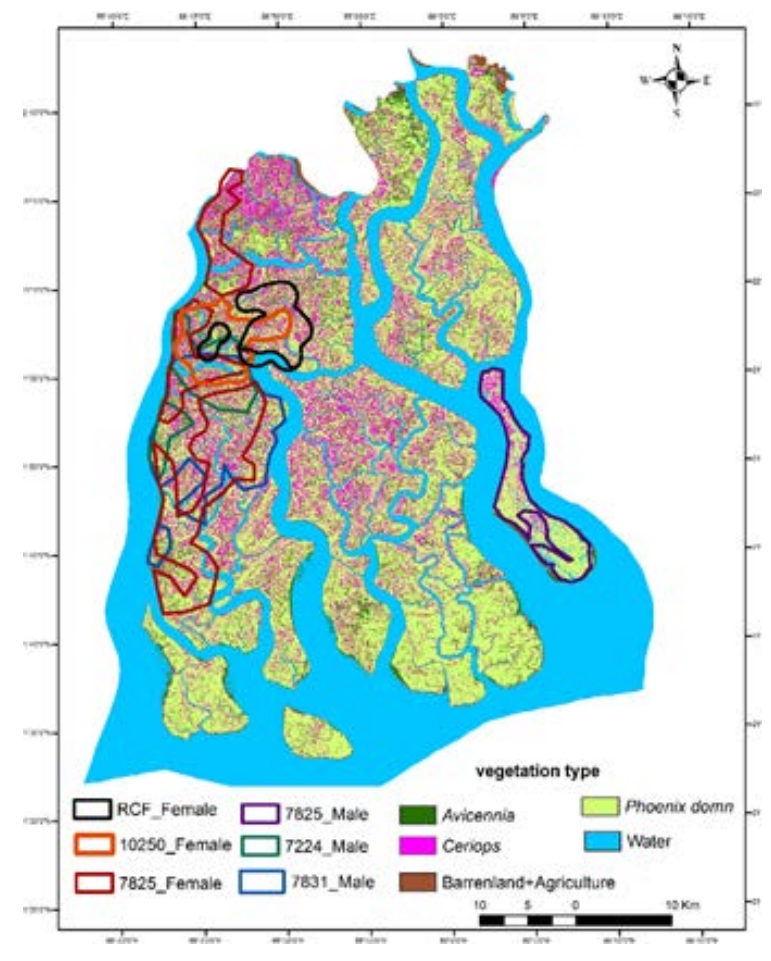

\section{Factors affecting tiger population in Sundarbans}

Plenty of prey animals, relatively ease in movements and trapping animals, safety from wave of the sea, security from human aggression and availability of fresh water play a determining role in tiger population (Bhattacharya, 2011).Tiger regulates their pray population consequently altering the structure and the function of the entire ecosystem (Bhattarai, 2009). Changes of climate resuts in rise of sea level that in turn pose a real fear of fragmenting the tiger habitat of Sundarban (Naha et al., 2016). Dube, 2021 analyzed the social factors affecting the conservation of tigers Sundarban and concluded that though the local people had standard awareness regarding tiger conservation but are not financially equipped to pay taxes or contribute towards the cause.

\section{Man-eating habit of the Tigers of Sundarbans}

Studies have directed that there is a direct co-relation between frequency of availability of human prey and man-eating habit of tigers in Sundarban (Bhattacharya, Jha \& Dave, 2001). Lack of wild prey, abundance of unprotected livestock causes tigers to attack villagers or livestock. (Lubis et al., 2020.) Bhattacharya, 2011, in their study noticed that that tigers attack men between 7.00A.M to 8.00 A.M in the morning and 3.00 P.M to 5.00 PM in the evening and this time interestingly coincides with the time the villagers go and return from their work, thereby concluding that the tigers have very good understanding of human nature. Das and Dutta, 2014 in their study observed that the tigers of Sundarban were generally seen to attack in the regions of higher salinity and thus established a positive correlation of salinity with the man-eating habit of tigers.

\section{Human tiger Conflict}

Human-tiger (Panthera tigris Linnaeus, 1758) conflicts (HTC), manifested primarily as attacks on people and domes-tic animals, exacerbate at least 2 major threats to tigers: (i) conflicts often result in mortality or removal of tigers from the wild; and (ii) they result in negative attitudes towards tigers by local people, thereby reducing support for tiger conservation.

Human-tiger (Panthera tigris Linnaeus, 1758) conflicts (HTC), manifested primarily as attacks on people and domes-tic animals, exacerbate at least 2 major threats to tigers: (i) conflicts often result in mortality or removal of tigers from the wild; and (ii) they result in negative attitudes towards tigers by 
local people, thereby reducing support for tiger conservation.

Human-tiger-conflicts (HTC), refers primarily as attacks on people and lifestock animals. HTC not only results in harm to man and animal, but also poses major threats to tigers. Such conflicts may cause either death or removal of tigers from the wild (Figure 3). They also cause negative attitudes towards tigers by local people, thereby reducing support for tiger conservation (Goodrich, 2010). Tiger population of Sundarban is well known for the highest expanse of conflict between tiger and human in the world. Unlike now, historically livestock rearing was not considered as important livelihood option in Sundarban, thus with the increase in lifestock in recent years tigers in conflict with humans result in their persecution (Naha et al., 2016). Tiger also kills fishermen while fishing in the rivers of the sundarbans; often tigers escape from the forest area and enter in the locality in search of food and killing domestic animals. (Ghosh, 2015). Like other parts of India people in Sundarbans generally do not hunt of kill tigers for pleasure or sport, mainly due to religious reasons and age-old beliefs. As a result, the tigers do not fear humans, do not relate them with terror and even consider them as potential prey. (Naha et al., 2016).

\section{Threats to tigers}

The increase in human population has resulted in urbanization which in turn has caused increased forest patchiness and natural habitat encroachment of tigers, reserve forests are now surrounded by human settlement and thus the tigers are forced to occupy spaces adjacent to human habitations (Wang et al., 2020). Killing of tigers and their prey, sea-level rise, wood collection were the highest ranked threats to the tigers (Aziz et al., 2013). Tigers are being hunted by humans for their skins and purported medicinal values, also losing natural habitat to deforestation and conversion to agricultural land which in turn results in great threat to the survival of Bengal Tigers (Bhattarai, 2009).Regular infiltration of NTFP collectors inside core areas and most importantly the changing character of the fragile eco-climatic condition of Sundarban also serve as threats to tiger population of Sundarban.

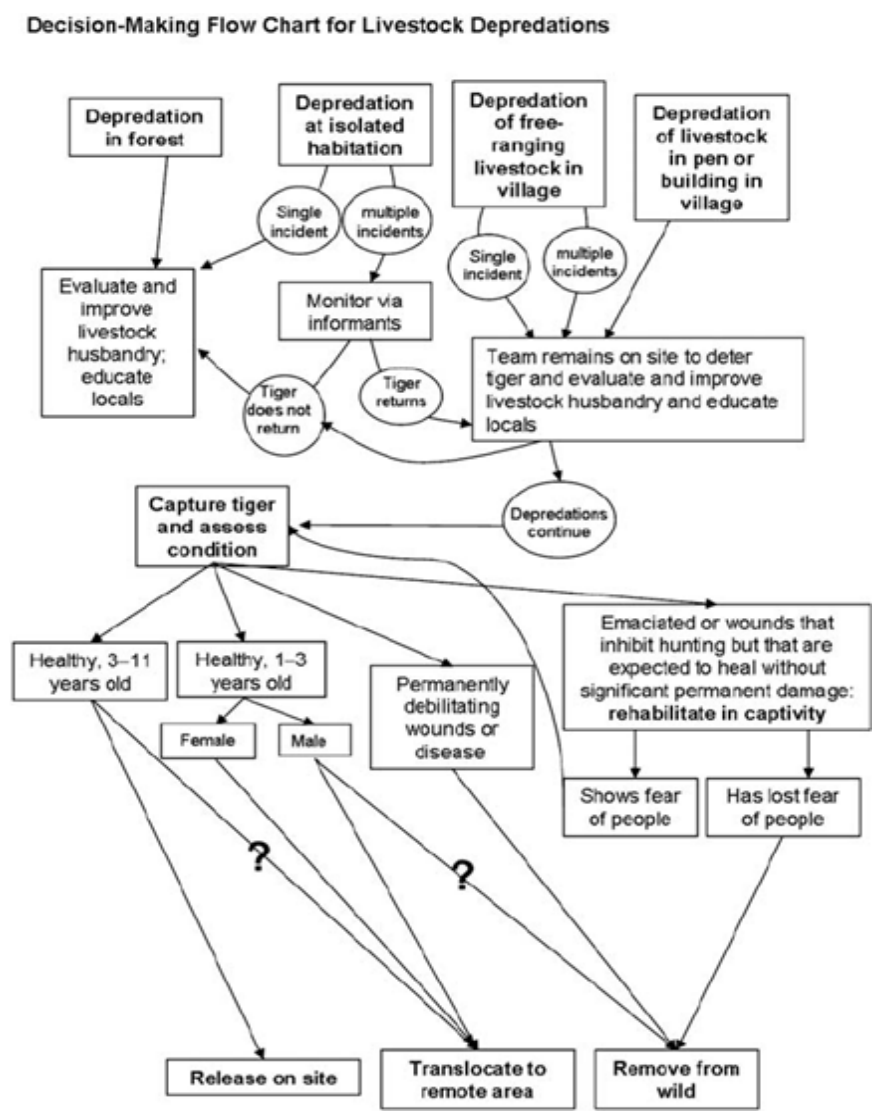

Figure 3: Decision Making Flowchart for Trained Tiger Response Team to be Undertaken During HTC (source: Wildlife Conservation Society, 2021). 


\section{Conservation of Royal Bengal Tiger and Tiger Project}

Royal Bengal Tigers are in a precarious situation (Bulte \& Damania, 2008). A stop on tiger shooting was imposed in 1970, and Project Tiger was launched in 1973, in nine reserve forests (Chakrabarti, 2009). Sundarban Tiger Reserve was also established in the same year which aim to preserve the natural habitat through stabilization of the natural mangrove ecosystem and conserve Royal Bengal Tiger and its associated wildlife in situ through restoration. IUCN has categorized tigers as 'endangered' animal in the Red Data Book. The monitoring of tiger along with its co-predators, prey and habitat in Sundarban was conducted as a part of All India Estimation of Tiger and these activities are prescribed by Project Tiger, Ministry of Environment and Forests (Bhattacharya, 2011).

\section{DISCUSSION}

The mystic Sunderbans with its unique natural beauty and diverse flora fauna is in danger. This world heritage site is under the threat of physical disaster, resulting in danger of the life it supports mainly the Royal Bengal Tiger. The results acquired so far in case of both ex-situ and in-situ conservation processes as adopted in the zoos and in Sundarban mangrove area respectively in West Bengal indicates that both the techniques which are mentioned that is essential and encomiastic to each other for successful stabilization of the prestigious and flagship animal i.e., Royal Bengal Tiger along with harmonious co-existence of other indigenous species in the natural ecological background (Dey, Debnath \& Sikdar, 2006) It is not a matter of certain speculation as to whether the problems experienced by the tiger widows of Sundarban will attack any attention in a national or indeed, international organization, even given the context of increasing HTC in recent years (Chowdhury et al., 2016). To reduce human-tiger conflict people should be provided with the education of conservation to make them aware about tiger behavior and ecology, regular monitoring of problem tigers to alert the people and compensation to losses can be helpful in this matter. (Bhattarai, 2009).

\section{CONCLUSION}

The conservation strategies undertaken by government and social bodies have shown positive results. According to the West Bengal Forest Department data, the tiger count for the year 2019-20 rose to 96 , from 88 in 2018-19. Thus, to conclude appropriate policies of sustainable intervention guided by a thorough research and followed by proper implementation, control and critical monitoring for necessary changes in action, is required to secure the biosphere drawing proper and sincere concerns of local people, regional and national governments and the world forums.

\section{ACKNOWLEDGEMENT}

The authors express their sincere thanks to Department of Biotechnology, Government of India for the funding from DBT-STAR College grant, under which this review project was conducted. We are also grateful to the Principal and the DBT-STAR Coordinator, Surendranath College, for their support and encouragement in implementing this review project at undergraduate level.

\section{REFERENCES}

Ashraf, M. A. (2010). The eco-political perspective: tiger conservation in Bangladesh as case study. Tigerpaper, 37(4), 20-24.

Azad, M. A. K., Hashem, M. A., \& Hossain, M. M. (2005). Study on human Royal Bengal tiger Interaction of in situ and ex situ in Bangladesh. J Biol Sci, 53, 250-2.

Aziz, A., Barlow, A. C., Greenwood, C. C., \& Islam, A. (2013). Prioritizing threats to improve conservation strategy for the tiger Panthera tigris in the Sundarbans Reserve Forest of Bangladesh. Oryx, 47(4), 510-518.

Bhattacharya, A. K., Jha, S., \& Dave, A. (2001). Biodiversity conservation and ecotourism; lessons from Sundarban tiger reserve. Van Vigyan-Jour For Sci, 39, 73-81.

Bhattacharya, S. (2011). Statue of faunal status with special reference to Bengal Tiger (Panthera tigris 
tigris) in Sundarban Delta. Exploratory animal and medical research, West Bengal Veterinary Alumni Association, West Bengal. India, 1, 41-52.

Bhattarai, B. R. (2009). Human-Tiger (Panthera tigris tigris) conflict in Bardia national park, Nepal. $A$ Thesis for Partial Fulfilment of Master of Science.

Bulte, E., \& Damania, R. (2008). Resources for sale: corruption, democracy and the natural resource curse. The BE Journal of Economic Analysis \& Policy, 8(1).

Chakrabarti, R. (2009). Local people and the global tiger: An environmental history of the sundarbans. Global Environment, 2(3), 72-95.

Chowdhurym, A. N., Mondal, R., Brahma, A., \& Biswas, M. K. (2016). Ecopsychosocial aspects of human-tiger conflict: an ethnographic study of tiger widows of Sundarban Delta, India. Environmental Health Insights, 10, EHI-S24899.

Das, G. K., \& Datta, S. (2016). Man-made environmental degradation at Sunderbans. Reason- $A$ Technical Journal (Formerly Reason-A Technical Magazine), 13, 89-105.

Dey, S., Debnath, H. S., \& Sikdar, P. K. (2006). A review of the legal tools for management of sundarban biosphere reserve, west bengal india. Indian Forester, 132(10), 1343.

Dube, P. P. (2021). Social Factors Affecting the Conservation of Tigers in the Samsher Nagar Area of Sunderbans. Asian Journal of Biology, 12-23.

Ghosh, P. (2015). Conservation and conflicts in the Sundarban biosphere reserve, India. Geographical Review, 105(4), 429-440.

Goodrich, J. M. (2010). Human-tiger conflict: a review and call for comprehensive plans. Integrative Zoology, 5(4), 300-312.

Kumar, S. (2020). Human-Tiger Conflicts a Current issue of Central India, Paradigm to Rethink the Consequences and Challenges of changing behaviour of Wild Animals in Human Dominated Landscape. Int. J. Sci. Res. in Biological Sciences Vol, 7(4).

Lubis, M. I., Pusparini, W., Prabowo, S. A., Marthy, W., Andayani, N., \& Linkie, M. (2020). Unraveling the complexity of human-tiger conflicts in the Leuser Ecosystem, Sumatra. Animal Conservation, 23(6), 741-749.

Mallick, J. (2012). Status of the mammal fauna in Sundarban Tiger Reserve, West Bengal-India. TAPROBANICA: The Journal of Asian Biodiversity, 3(2).

Mallick, J. K. (2013). Ecology, status and aberrant behavior of Bengal Tiger in the Indian Sundarban. Animal Diversity, Natural History and Conservation, 2, 381-454.

Naha, D., Jhala, Y. V., Qureshi, Q., Roy, M., Sankar, K., \& Gopal, R. (2016). Ranging, activity and habitat use by tigers in the mangrove forests of the Sundarban. PLoS One, 11(4), e0152119.

Raha, A. K., \& Saha, B. K. (2004). A Wonder that is Sundarban. Kolkata, India: Computronics.

Wang, C. C., Kwan, K. Y., Shin, P. K., Cheung, S. G., Itaya, S., Iwasaki, Y., ... \& Hsieh, H. L. (2020). Future of Asian horseshoe crab conservation under explicit baseline gaps: A global perspective. Global Ecology and Conservation, 24, e01373.

Wildlife Conservation Society. (2021). https://www.wcs.org/ 


\title{
Impacts of Covid-19 Pandemic
}

\author{
Aslam Khan, Titli Mistry, Tarikul Islam Golder* \\ Department of zoology, Surendranath College, Kolkata, India \\ *Corresponding Author’s Email: tarikulgolder@gmail.com
}

\begin{abstract}
The Corona infection or COVID-19 is a sickness dependent on an obscure infection. It appears to be that it began in China and has broadly spread in practically all nations on the planet. This pandemic circumstance is one of the broadly spread infections in late history. Nonetheless, there was flu pandemic in 1918 with the specific number of passing as yet unclear. Some accept that the loss of life would have been around 50-100 million individuals. The article is pointed toward examining the positive and adverse consequences of COVID-19 in a sociological viewpoint. It is additionally centered around potential difficulties to the store network in South Asia. South Asian nations are exceptionally affected by the pandemic circumstance, and the local portrayal is about $4 \%$ in the later piece of May 2020 with an expanding propensity. Additionally, the article has a proposition for the control of the infection just as the whole financial, natural and political environment in a country, while especially giving more weight to South Asia. The proposed activities are examined in present moment, mid-term and long-haul premise, and any master and social laborer who is engaged with the pandemic control cycle can acquire an understanding into what to do and how to play out their errands. A sociological examination on COVID-19 is vital on the grounds that there is a wing involving prevailing clinical specialists in the control and the executives of the infection. The article stresses the significance of a sociological examination in a pandemic circumstance. Normally, anybody would think about a pandemic circumstance in exceptionally adverse terms because of its enthusiastic, financial, natural, political and social elements. Nonetheless, it is likewise sure because of specific factors that assistance to reintegrate and rearrange the social framework all in all.
\end{abstract}

Keywords: Covid-19; Pandemic; Sar-Cov-2; Influenza; WHO

\section{INTRODUCTION}

An intense respiratory contamination of obscure beginning was first detailed in December 2019, in Wuhan, China, called novel Covid tainted pneumonia (NCIP). Later on, the microbe was distinguished as an original wrapped RNA B-Covid, using fair-minded sequencing, which was of comparable phylogeny to SARS-CoV. On January 12, 2020, WHO named this new infection as the 2019. On January 30, 2020, WHO declared this infection as a worldwide pandemic (WHO, 2020 a). On February 11, 2020, WHO named the original sickness as Corona Virus Disease 2019 (COVID-19) (WHO, 2020 b). The Corona infection Study Group (CSG) named this infection as serious intense respiratory condition Covid 2 (SARS-CoV-2). The SARS-CoV-2 can influence the respiratory parcel including nose, mouth, throat, sinuses and lungs. Human-to human and human-to-creature transmissions have been assumed for this infection. Respiratory drops of the tainted individual are the most probable instruments of transmission. These drops convey the infection into the air and can go into a host body through nose and mouth. The infection causes aggravation in lungs which harm the aspiratory cells by starting a fiery response. Heaving, looseness of the bowels, weariness and high fever might create with extreme aggravation (Guo et al., 2020). Fiery liquid delivered because of aggravation fill the lungs, bringing about hacking and trouble in breathing by decreasing the capacity of alveoli. The oxygen level in the blood might drop while different organs, for example, kidneys or the heart may be influenced in serious cases (Geier \& Geier, 2020; Garcia et al., 2020). The beginning of this disease was Wuhan city from where it spreads to the remainder of the world (Lu, Stratton \& Tang, 2020). This illness has imagined a huge danger to the wellbeing and economy of the world. 
Impacts of Covid-19 Pandemic

\section{LITERATURE REVIEW}

\section{Negative impacts of coronavirus in our life:}

\section{Unemployment}

Financial analysts said the current number of jobless doesn't consider millions who have joined the rural area as occupations evaporated in the remainder of the economy (Blustein et al., 2020). While these individuals are formally considered utilized, the misfortune in wages and purchasing influence could comprise "masked" joblessness. The basic issue in India is that the typical business computing philosophy has separated amidst pandemic," political financial specialist M K Venu told DW. Overviews show out of an employable labor force of 520 million, almost 35 to $40 \%$ (or up to 200 million specialists) might be underemployed with no genuine work or utilized yet acquiring half or less of their previous compensation in metropolitan places," he said. This is exceptional in light of the fact that the authority joblessness rate is around $8 \%$, yet this doesn't consider huge underemployment and individuals professing to be utilized yet with half or less compensation.

\section{Suicide}

Numerous Indian COVID-19 self-destruction cases are turning the press-media consideration and flooding in the web-based media stages albeit, no specific examinations surveyed the COVID-19 selfdestruction causative components generally. Accordingly, the current examination presents 269 COVID-19 self-destruction cases (matured 19 to 65 years). The self-destruction causalities are incorporated as follows - dread of COVID-19 contamination, trailed by monetary emergency, dejection, social blacklist and strain to be isolate, COVID-19 positive, COVID-19 business related pressure, incapable to return home because of lockdown, inaccessibility of liquor and so on Considering the super mental effects identified with COVID-19, there arises a requirement for countrywide broad tenemental wellbeing (Gunnell et al., 2020).

\section{School dropout}

Right around 24 million youngsters are in danger of not getting back to class one year from now because of the financial aftermath of COVID-19, as indicated by the United Nation's approach brief on the pandemic's effect on training (World Bank, 2020). The instructive financing hole is likewise prone to increment by $33 \%$, it said. More than 1.6 billion students across the world have been influenced by the interruption of the instruction framework, however the pandemic has likewise served to worsen existing variations, with weak populaces in low-pay nations taking a harder and longer hit. For instance, during the second quarter of $2020,86 \%$ of kids at the essential level have been adequately out of school in helpless nations, contrasted with only $20 \%$ in exceptionally created nations.

\section{Obesity among child and elderly people}

The Covid prompted lockdowns across the world have adversely affected eating regimen, rest, and actual work among kids with corpulence, a new report clarified (Zemrani et al., 2021). As indicated by University at Buffalo research, the investigation, distributed in April in Obesity, analyzed 41 overweight kids under imprisonment all through March and April in Verona, Italy. Contrasted with practices recorded a year earlier, the youngsters and the older people groups ate an extra feast each day; dozed an additional half hour out of every day; went through almost five hours of the day before the telephone, PC and TV screens; and drastically expanded their utilization of red meat, sweet beverages, and lousy nourishments. Active work, then again, diminished by over two hours of the week, and the measure of vegetables devoured stayed unaltered.

\section{Educational system}

The effect of pandemic COVID-19 is seen in each area all throughout the planet. The training areas of India just as world are severely influenced by this. It has authorized the overall lock down making exceptionally terrible impact on the understudies' life. Around 32 crore students halted to move schools/universities and all instructive exercises ended in India. The flare-up of COVID-19 has 
instructed us that change is inescapable. It has functioned as an impetus for the instructive establishments to develop and decide on stages with advances, which have not been utilized previously. The instruction area has been battling to endure the emergencies with an alternate methodology and digitizing the difficulties to wash away the danger of the pandemic.

\section{Treatment problems}

The COVID-19 pandemic significantly affects the limit of wellbeing frameworks to proceed with the conveyance of fundamental wellbeing administrations. While wellbeing frameworks all throughout the planet are being tested by expanding interest for care of COVID-19 patients, it is basic to keep up with preventive and corrective administrations, particularly for the most weak populaces, like kids, more seasoned people, individuals living with persistent conditions, minorities and individuals living with handicaps. Nations need to accomplish the ideal harmony between battling the COVID-19 pandemic and upkeep of fundamental wellbeing administrations, said Dr Tedros Adhanom Ghebreyesus, Director-General of the World Health Organization. "Many individuals who need therapy for illnesses like malignancy, cardiovascular infection and diabetes have not been getting the wellbeing administrations and medications they need since the COVID-19 pandemic started. It's imperative that nations discover creative approaches to guarantee that fundamental administrations for NCDs proceed, even as they battle COVID-19.

\section{Impact on business}

The COVID-19 effect differs by sort of business, with these three classes generally influenced: individual help, friendliness and retail. The more modest the organization, the harder the hit, with organizations with less than 20 workers generally influenced. Since an independent company with less than 20 workers normally need income and capital. Those organizations were quick to diminish hours for representatives or lay off workers. Those organizations were additionally quick to quit employing subcontractors. "Those organizations had a test, and will encounter the most outrageous adverse consequence," said Laurie McCabe, SMB's fellow benefactor and accomplice. "They needed to figure out how to serve clients in the new stay-at-home climate. "McCabe said that proceeding to work has been inconceivable for private companies which offer active types of assistance, like boutiques and spas, home improvement and fix workers for hire, and for some dental and clinical workplaces.

\section{Possitve impacts of coronavirus in our life}

\section{On environment}

The overall disturbance brought about by the COVID-19 pandemic has come about in various; and 50 percent decrease in nitrogen oxides emanations which one Earth frameworks researcher assessed may have saved something like 77,000 lives more than two months. Other constructive outcomes on the climate incorporate administration framework controlled speculations towards a feasible energy progress and different objectives identified with natural insurance, for example, the European Union's seven-year $€ 1$ trillion spending proposition and $€ 750$ billion recuperation plan "Cutting edge EU" which looks to save $25 \%$ of EU spending for environment amicable consumption. Notwithstanding, the flareup has additionally given cover to criminal operations, for example, deforestation of the Amazon tropical jungle and poaching in Africa, thwarted ecological strategy endeavors, and made financial drop out that some foresee will slow interest in environmentally friendly power energy Technologies (Gardiner, 2020).

\section{Road accidents decreases}

One beneficial outcome of measures executed to control the spread of COVID-19 was the decrease of auto collisions, on both metropolitan and interurban streets. This brought about a stamped fall in the quantity of traffic related wounds and fatalities, as seen by. Among the principle factors behind car crashes are natural conditions (climate, perceivability, and so on), street attributes, vehicle plan, human blunder, and the volume of vehicles flowing on our streets. At times, auto collisions are because of a mix of more than one of the above mentioned. The likelihood of auto collisions happening 
increments with the volume of vehicles on our streets and in accordance with certain street attributes. This likelihood expansions in circumstances portrayed by both low and undeniable degrees of clog. In the last case, nonetheless, the seriousness of the subsequent mishaps will in general fall when vehicles crash at lower speeds. The limitations on portability presented following the COVID-19 episode have suggested a sharp decrease in street traffic. This ought to have had an immediate and recognizable impact.

\section{Decrease of other infectious diseases}

During lockdown, due to imagining from Covid everybody was extremely delicate about there, they timely washed their hands with shop or hand washes. They utilized face cover as necessary while going external the house, they additionally kept up with social removing to imagine Covid. By this individual ready to imagine Covid as well as numerous irresistible sicknesses by the assistance of those genuine own wellbeing

\section{Positive impact on migratory birds and insects and marine animals}

Dissimilar to on account of people, the COVID-19 lockdown appears to emphatically affect creatures and birds. Restricted human impedance and diminished contamination levels in the locale have assisted them with recovering their space in the biological system partially, say naturalists. Many bird species which relocate from different pieces of the nation - known as incomplete transient birds - have now broadened their visit at the Therthangal and Melaselvanoor-Keelaselvanoor bird asylums, says Ramanathapuram Forest Range Officer S. Sathish. A.S. Marimuthu, Wildlife Warden, Ramanathapuram, says the lockdown time frame has helped in lessening wounds to marine creatures.

\section{Learning technology}

The COVID-19 has brought about schools closed the whole way across the world. Internationally, over 1.2 billion kids are out of the study hall. Subsequently, instruction has changed drastically, with the unmistakable ascent of e-learning, whereby educating is attempted distantly and on advanced stages. Examination proposes that internet learning has been displayed to expand maintenance of data, and take less time, which means the progressions Covid have caused may be setting down deep roots (Putra et al., 2020). As per the new discoveries from 48 nations in the OECD's Teacher and Learning International Survey, just $60 \%$ of instructors have gotten proficient improvement in the utilization of web and correspondence innovation, while near $20 \%$ of educators revealed a significant requirement for advancement around here.

\section{DISCUSSION}

When these positive and negative impacts are considered, it is very clear that COVID-19 has caused more positive impacts to the nations, regions and the world, particularly to South Asian countries. However, some countries such as Italy, Spain, the USA, and China and several European countries have suffered a lot (Business Standard, 2021). At the time of writing this article, COVID-19 has infected $5,306,928$ persons worldwide (when the article was finalised for publication, the number has increased up to 15,947,291). Comparatively, these positive and negative impacts are valid for these countries too, irrespectively of the level of the spread of COVID-19.

The proposed future action plan or COVID-19 recovery action plan mainly focuses on the socioeconomic, environment and political spheres and not on the medical and technical spheres.

At the point when these various spaces or circles are seen, it turns out to be exceptionally evident that there are bigger quantities of activities distinguished under friendly areas. It suggests that COVID-19 is a pandemic circumstance and it has more basic viewpoints in the social area which should be taken a gander at through a sociological as opposed to managerial or political point of view (Saladino, Algeri \& Auriemma, 2020). In addition, the majority of the proposed activities are centered around present moment and medium-term activities. Nonetheless, there are some critical activities recognized under long haul activities which are identified with individual and gathering level mentalities. 
This load of examinations done and expounded in various subsections of the paper feature that there is an essential job to be played by the inventory network organizations and the executives' bunches in each general public. In South Asian nations, the circumstance in inventory network organizations and the board is more explicit than in different regions of the planet since this load of nations keep a double method of economy-resource and business. Especially, end clients and shoppers are occupied with some homegrown or bungalow creation frameworks. On the off chance that they have an excess, they share it with their family members and neighbors as a social propensity. Along these lines, they are not absolutely reliant upon the market framework. Some of them offer these homegrown items to stores. Here and there they give some worth expansion as cabin items and attempt to support a steady production network organization. The present circumstance is seen in numerous South Asian nations. Accordingly, maintainability in production network networks in South Asian nations is exceptionally unstable. In any case, clearly the inventory network networks are compelling and of central significance under the COVID 19 pandemic circumstance in South Asian nations.

Particularly, if the specialists center around this COVID 19 recuperation activity plan, it will assist with securing the family, climate and the country. Along these lines, the authors need to have an attitudinal change towards a more feasible control of the pandemic circumstance in our country. The whole examination of this paper is essentially founded on the specialist's Sri Lankan and other South Asian nations' experience, especially being developed arranging. The knowledge will assist with overseeing and control the COVID-19 pandemic circumstance in South Asian nations. In case the authors can adjust to the circumstance as clarified, this methodology will assist them with keeping a steady and maintainable improvement program for these nations, especially for Sri Lanka.

When contrasted with a few verifiable encounters in overseeing pandemic circumstances, this COVID19 pandemic circumstance can be utilized as an improvement freedom to partake in a lot higher takeoff with another arrangement of perspectives during the time spent turn of events.

\section{CONCLUSION}

The current examination adds to our comprehension of the effect of the COVID-19 emergency on work and private life. It gives proof on the covariates of a more regrettable/positive saw sway and on the relationship with MWB and SRH in the German and Swiss working populaces. Representatives whose business contract was influenced by the emergency appear to contrarily affect their work life. This features the vital job of (un-/under-) work in an emergency, as business is related with a few wellbeing advancing components that can't be subbed in some other manner. Besides, the private everyday routine of representatives experiencing alone has been influenced most contrarily because of social disconnection. Consequently, mental medical aid additionally open online ought to be set up especially for these weak gatherings. Businesses need to guarantee that they keep close friendly binds with and sincerely support representatives with decreased agreement or working hours. In addition, fast monetary guides are expected to the individuals who have lost their pay somewhat or totally. By the by, we should likewise cultivate positive outcomes of the emergency. By and large, it appears to be that an increment in WFH was positive for work life. Gaining from the useful impacts of WFH in an emergency can illuminate future authoritative and administrative strategies to help this type of working. As representatives experienced with WFH decidedly affected private life than beginners, future WFH approaches ought to incorporate contribution preparing and trade of involvement between workers on the most proficient method to set up certain schedules viable with their private life. This will assist representatives with proactively distinguishing their inclinations and specialty their workplace as needs be. Further, an increment in relaxation time was especially certain for private life. More relaxation time takes into account devoting additional chance to exercises one appreciates, and this might be valuable likewise for recuperation and separation from work and for emotional well-being overall. Consequently, representatives could likewise be prepared in ideal creating of their relaxation time to fortify these advantageous impacts. At long last, we saw that other than the announced genuine changes in work and private life, likewise the view of the general positive or adverse consequence is identified with the wellbeing results. This proposes to offer positive brain science trainings to workers assisting them with intentionally zeroing in on and utilize expected positive results of the emergency. From a longitudinal examination point of view, it is fascinating to additionally analyze how the real and saw effect of the 
continuous emergency just as the related wellbeing results change over the long run and regardless of whether a portion of the new schedules created during the pandemic will be kept up with in the long haul. To finish up, our examination adds to ongoing proof that the Covid-19 emergency and related lockdown measures don't have exclusively adverse consequence. Maybe, it influences weak gatherings of people who need designated support, while most of the populace stay sound or even experience positive changes in their everyday life.

\section{ACKNOWLEDGEMENT}

The authors express their sincere thanks to Department of Biotechnology, Government of India for the funding from DBT-STAR College grant, under which this review project was conducted. We are also grateful to the Principal and the DBT-STAR Coordinator, Surendranath College, for their support and encouragement in implementing this review project at undergraduate level.

\section{REFERENCES}

Blustein, D. L., Duffy, R., Ferreira, J. A., Cohen-Scali, V., Cinamon, R. G., \& Allan, B. A. (2020). Unemployment in the time of COVID-19: A research agenda.

Business Standard (2021). China Locks Down Thousands in North to Contain Latest Covid-19 Outbreak. $25^{\text {th }}$ October. https://www.business-standard.com/article/current-affairs/china-locksdown-thousands-in-north-to-contain-growing-delta-outbreak-121102500292_1.html

Garcia, E. M., Caballero, V. R., Albiach, L., Aguero, D., Ambrosioni, J., Bodro, M., ... \& Soriano, A. (2020). Tocilizumab is associated with reduction of the risk of ICU admission and mortality in patients with SARS-CoV-2 infection. medRxiv.

Gardiner, B. (2020). Pollution made COVID-19 worse. Now lockdowns are clearing the air. National Geographic.[Website Link].

Geier, M. R., \& Geier, D. A. (2020). Respiratory conditions in coronavirus disease 2019 (COVID-19): Important considerations regarding novel treatment strategies to reduce mortality. Medical Hypotheses, 140, 109760.

Gunnell, D., Appleby, L., Arensman, E., Hawton, K., John, A., Kapur, N., ... \& Yip, P. S. (2020). Suicide risk and prevention during the COVID-19 pandemic. The Lancet Psychiatry, 7(6), 468-471.

Guo, Z. D., Wang, Z. Y., Zhang, S. F., Li, X., Li, L., Li, C., ... \& Chen, W. (2020). Aerosol and surface distribution of severe acute respiratory syndrome coronavirus 2 in hospital wards, Wuhan, China, 2020. Emerging infectious diseases, 26(7), 1586.

Lu, H., Stratton, C. W., \& Tang, Y. W. (2020). Outbreak of pneumonia of unknown etiology in Wuhan, China: The mystery and the miracle. Journal of medical virology, 92(4), 401.

Putra, P., Liriwati, F. Y., Tahrim, T., Syafrudin, S., \& Aslan, A. (2020). The Students Learning from Home Experience during Covid-19 School Closures Policy in Indonesia. Jurnal lqra, 5(2).

Saladino, V., Algeri, D., \& Auriemma, V. (2020). The psychological and social impact of Covid-19: new perspectives of well-being. Frontiers in psychology, 11, 2550.

WHO, G. (2020). Statement on the second meeting of the International Health Regulations (2005) Emergency Committee regarding the outbreak of novel coronavirus (2019-nCoV). World Health Organization.

World Bank. (2020). The COVID-19 pandemic: Shocks to education and policy responses.

World Health Organization. (2020). Naming the coronavirus disease (COVID-19) and the virus that causes it. Brazilian Journal of Implantology and Health Sciences, 2(3).

Zemrani, B., Gehri, M., Masserey, E., Knob, C., \& Pellaton, R. (2021). A hidden side of the COVID-19 pandemic in children: the double burden of undernutrition and overnutrition. International Journal for Equity in Health, 20(1), 1-4. 


\title{
Inorganic Nanoparticles
}

doi: $10.46977 /$ book.2021.rabs

\author{
Atanu Ganguly, Anirudha Bhattacharjee, Shourya Ghosh, Apurba Biswas* \\ Department of Chemistry, Surendranath College, Kolkata, India
}

${ }^{*}$ Corresponding Author's Email: apurbacu@yahoo.co.in

\begin{abstract}
Inorganic nanomaterials, including gold nanoparticles, silver nanoparticles, iron nanoparticles, silica nanoparticles, and magnetic nanoparticles, are non-toxic, have a tendency to mix, and are highly stable compared to organic materials, as demonstrated in recent investigations. In the field of modern material science, inorganic nanoparticles are being developed as novel drug delivery systems because of their own physical properties, which include size-sensitive optical, magnetic, electronic, and catalytic properties. These nanoparticles have many physical properties, including high stability, high surface area, tunable compositions, and abundant physicochemical multifunctionality. The size, morphology, and surface charge of nanoparticles are commonly measured using nuclear magnetic resonance, dynamic light scattering, optical microscope, atomic force microscopy and electron microscopy.
\end{abstract}

Keywords: Nanoparticles; Gold; Silver; Iron; Silica; Characterization

\section{INTRODUCTION}

Inorganic nanoparticles are one of the most fundamental nanomaterials in modern technologies because they can be easily synthesized and mass produced at a low cost, so they are often used in applications. It also gets much more attention for drug or gene delivery carriers as they have markedly different physical, chemical, and biological properties from their bulk counterparts. Shape and size can have a strong impact on the electromagnetic, optical, and catalytic properties of noble metal nanoparticles, such as gold, silver. By convention, 1-100 nm is the nanoscale most often used in reference to nonmaterials (Pandey \& Dahiya, 2016), but there is no clear explanation from a biological or chemical perspective (Laurent et al., 2010). This review covers pharmaceutically important inorganic nanoparticles. These nanostructures can be made from metals, metal oxides, carbon, and other types of organic materials. Silver nanoparticles (AgNPs), silicon nanoparticles (SNPs), gold nanoparticles (GNPs), quantum dots (QDs) and other carbon-based nanoparticle and biopolymer nanostructures are the most common types used in the nonmedical field. In the field of chemistry, it has both advantages and disadvantages if it is to determine how to use something for a purpose. For example, silicon nanostructures are generally used as a support for drug loading, while gold nanoparticles are mainly used with photo thermal agents in cancer treatment and biosensing.

\section{LITERATURE REVIEW AND DISCUSSION}

\section{Metallic inorganic nanoparticles}

Metal nanoparticles are submicron-sized self-contained creations made of different pure metals such as gold, silver, platinum, zinc, etc. or their complexes such as hydroxides, phosphates etc. (Mody et al., 2010). Metal NPs are very harmful to living cells, so the creation and use of powder form metal NPs is considered to be dangerous (Pandey \& Dahiya, 2016). Metal NPs have been used widely in biomedical and engineering field as well as it has enormous impact in our daily life. Antibodies, drugs can be easily bind with different functional groups that can be introduced in the metal NPs. The development of new, economically viable methods for the production of MNPs has led to pilot-scale production of MNPs, which are now widely used in footwear, clothing, cosmetics, shampoos, and plastic containers. Metallic nanoparticles are one of the most important research issues and have 
proven themselves to be extremely effective in biomedical sciences and engineering.

\section{Gold nanoparticles (GNPs)}

GNPs are tiny gold particles with a diameter of 1 to $100 \mathrm{~nm}$ (Pandey \& Dahiya, 2016). They are also known as colloidal gold when they are distributed in water. GNPs are found in a wide range of sizes, from 2 to $100 \mathrm{~nm}$ (Jadzinsky et al., 2007). The 20-50 nm particle size ranges showed the greatest cellular uptake. 40-50 nm particles have been shown to cause specific cell damage (El-Sayed, Huang \& El-Sayed, 2006). These $40-50 \mathrm{~nm}$ particles can diffuse into tumors, but they can be easily removed, and the patient can quickly return to a normal state of health, mind, or both. A bigger particle, 80-100 $\mathrm{nm}$, on the other hand, does not spread into the tumor over a large area, and instead remains in the same area near the blood vessels. For verification of Monte Carlo simulations, different sizes of nanoparticles can be prepared in experimental gold nanoparticle micro dosimetry. The citrate reduction technique is used to produce gold nanoparticles.

\subsection{Application of gold nanoparticles:}

Gold nanoparticles, most stable metal nanoparticles have huge importance in this emerging nanotechnology and medical field. These nanoparticles have been carefully designed to be used as conductors in everything from printable inks to electronic chips (Huang et al., 2003). Photodynamic therapy, photothermal therapy as well as radiation therapy are the most useful methods for the treatment of different types of cancer because of near-IR-absorbing gold nanoparticles produce heat for their excitation by light at wavelengths of 700 to $800 \mathrm{~nm}$. These high intensity radiations allow these nanoparticles to kill targeted tumors (Stuchinskaya et al., 2010).

\section{Silver nanoparticles ((AgNPs)}

Silver nanoparticles (AgNPs) are one of the most important and extremely interesting nanomaterials, among many metallic nanoparticles, that play a role in biomedical applications (Pandey \& Dahiya, 2016). AgNPs are essential to nanoscience and nanotechnology, specifically nanomedicine (Gurunathan et al., 2015). Silver nanoparticles are small silver particles of 1-100 mm in size that are rapidly used as silver because of their high surface-to-volume ratio. Silver nanoparticles (AgNPs) are increasingly used in different industries, including optical, electrical, and thermal properties, as well as biological properties.

\subsection{Application of silver nanoparticles}

(i) Antibacterial agents: Silver nanoparticles can be used in consuming and medical products to sterilize nanomaterial, such as medical, consumer, textiles, food and health care products (Keat et al., 2015). These nanoparticles have the ability to change structure of cell membranes as well as to penetrate bacterial cell walls, causing cell death.

(ii) Sensors: In recent years, silver nanoparticles have been used for sensing and imaging applications due to their valuable optical properties. Colorimetric sensing of peptide-capped silver nanoparticles has been extensively studied, with a focus on the interaction of peptide and the silver nanoparticles (Bansal \& Verma, 2014).

\section{Iron oxide nanoparticles (INPs)}

Iron oxide nanoparticles are radish brown paramagnetic inorganic compounds, having the diameter 1100 nanometers with two general forms magnetite $\left(\mathrm{Fe}_{3} \mathrm{O}_{4}\right)$ and its oxidized form maghemite $\left(\mathrm{\gamma}-\mathrm{Fe}_{2} \mathrm{O}_{3}\right)$ (Pandey \& Dahiya, 2016). Recently, iron oxide nanoparticles have gained much attention for their remarkable new properties, such as super paramagnetism, high field irreversibility, surface-to-volume ratio, higher surface area, high saturation field and easy separation method (Elias \& Tsourkas, 2009; Peng etal., 2008).

\subsection{Applications of iron nanoparticles:}

Iron nanoparticles have wide range of applications in industrial and medical field. Used to clean 
industrial sites contaminated by chlorinated organic compounds, this compound is found in plastics, nanowires, coatings, nanofibres, and textiles. These nanoparticles are expected to most promising application in targeted drug delivery system due to their response to magnetic field.

\section{Silica nanoparticles}

Colloidal Silica (silicon dioxide) nanoparticles are amorphous materials that are commonly shaped like a sphere. They are available in a wide variety of sizes, and their surface chemistry can be adapted to suit a range of applications. Sober and coworkers first made tiny silicon particles of $1 \mathrm{~mm}$ in size using sol-gel method and then systematically characterized them. Bogus et.al. have performed such extensive research on controlling hydrolysis of silica nanoparticles of a few hundred nanometers to several micrometers in size. In medical imaging, silicon nanoparticles serve as a crucial aid and support function, and they are used to encapsulate contrast agents' particles, such as organic dyes, quantum dots.

\subsection{Application of silica nanoparticles}

Silica nanoparticles have numerous applications, including pharmaceutical delivery and theranostics. They can be used as an additive to produce rubber and plastics, as a strong filler for concrete and other construction composites, and as a firmly fixed, non-poisonous platform for biomedical applications.

\section{Nanoparticle characterization techniques}

Nuclear magnetic resonance (NMR), optical microscope, electron microscope such as scanning electron microscope (SEM), atomic force microscopy (AFM), field emission scanning electron microscope (FESEM), tunneling electron microscope (TEM), dynamic light scattering (DLS), etc. have been commonly used to measure the size, morphology, and surface charge of nanoparticles.

\subsection{Nuclear magnetic resonance (NMR):}

Nuclear magnetic resonance (NMR) spectroscopy is an analytical technique for the structural determination of nano materials and it also used in the quantative determination of nanoparticles. It is based on a phenomenon in which nuclei possess nonzero spin in a strong magnetic field that supplied from outside. This magnetic field causes the energy difference between the aligned and opposed orientations of spins i.e. 'spin-up' and 'spin-down' states. It is used to study surface chemistry and the physical properties of the nanomaterials due to their interaction between ligand and magnetic surfaces of nanoparticles although it is not suitable for characterizing ferro or ferrimagnetic materials. Chemical shifts enhance the selectivity and allow determining the physicochemical characteristics of components in the inorganic nanoparticle (Chow, 2003).

\subsection{Optical microscope}

Through the magnifying of a lens with visible light, optical microscopy is a method designed to take a closer look at a small part or quantity, to show what the whole looks like. Most nanoparticles are below the contrast visual representation limit of the best resolution that possible of an optical microscope; therefore, it has limitations to observe different features of nanostructured materials in details. Plasmonic grating can be used as adapter to bring down the resolution and then it can be used for nanoscale microscope technique, though microscopy can still be useful to measure the approximate size and crystallinity of starting materials, as might be needed in mixing or homogenization processing, or other larger particles.

\subsection{Electron microscopy}

Different types of electron microscopy are used to characterize nanoparticles, among them scanning electron microscopy (SEM) and transmission electron microscopy (TEM) is being widely used. It is a high-resolution technique in which electrons are used instead of photon to analyze the nanoparticles Scanning and transmission electron microscopy, respectively, allow direct observation of nanoparticles, while TEM is better for its narrower size limit as well as less backscattered. It also 
provides chemical and structural information of materials in nano scale. The freeze-fracture procedure, in which a cast is made of the real small part or quantity intended to show what the whole looks like, can result in very detailed contrast visual representation data, though lower vacuum (environmental or E-SEM) instrumentation can limit this manipulation at the expense of some resolution (Kelly et al., 2003).

\subsection{Dynamic light scattering (DLS)}

Dynamic light scattering is a very useful technique for characterizing nanoparticles and can be used to determine the diameter of the particles that move in different directions in a colloidal solution. This method is based on diffusion motion of the particles in which larger particles scatter more compare to smaller particles due to their different Brownian motion in liquid solution. It can be used not only for measuring the size but also the stability over time in different $\mathrm{pH}$ medium and temperature conditions. Information of surface electric charge as well as stability of the nanoparticles can be obtained with the help of Zeta potential. Photon correlation spectroscopy (PCS) or dynamic light scattering are the fastest and most widely used methods of calculating minute particle sizes, and they currently rank in the first place. (deAssis et al., 2008).

\subsection{Atomic force microscopy (AFM)}

Atomic force microscopy is used to characterize nanoparticles with sub nanometer particles size. It is a very good characterization technique compare to other techniques such as optical microscope, electronic microscope and dynamic light scattering. Therefore, it becomes very popular one. It is commonly used for surface analysis of nanoparticles to get high resolution images (Zur Mühlen et al., 1996).

\section{CONCLUSION}

Nanoparticles have wide range of applications specially it may be regarded as a part of a novel drug delivery system, which can play a crucial role in delivering drugs with greater therapeutic potential, better bioavailability, and minimal side effects and toxicity. It has made a profound contribution to the modern medical system, and it is expected to become a landmark in the emerging health care system. Nuclear magnetic resonance, optical microscope, electron microscopy, dynamic light scattering, and atomic force microscopy are commonly used for characterization of size, morphology, and surface charge of nanoparticles.

\section{ACKNOWLEDGEMENT}

The authors express their sincere thanks to Department of Biotechnology, Government of India for the funding from DBT-STAR College grant, under which this review project was conducted. They are also grateful to the Principal and the DBT-STAR Coordinator, Surendranath College, for their support and encouragement in implementing this review project at undergraduate level.

\section{REFERENCE}

Bansal, A., \& Verma, S. S. (2014). Searching for alternative plasmonic materials for specific applications. Indian Journal of Materials Science, 2014.

Chow, T. S. (2003). Size-dependent adhesion of nanoparticles on rough substrates. Journal of Physics: Condensed Matter, 15(2), L83.

de Assis, D. N., Mosqueira, V. C. F., Vilela, J. M. C., Andrade, M. S., \& Cardoso, V. N. (2008). Release profiles and morphological characterization by atomic force microscopy and photon correlation spectroscopy of $99 \mathrm{mTechnetium-fluconazole} \mathrm{nanocapsules.} \mathrm{International} \mathrm{Journal} \mathrm{of}$ Pharmaceutics, 349(1-2), 152-160.

El-Sayed, I. H., Huang, X., \& El-Sayed, M. A. (2006). Selective laser photo-thermal therapy of epithelial 
carcinoma using anti-EGFR antibody conjugated gold nanoparticles. Cancer Letters, 239(1), 129135.

Elias, A., \& Tsourkas, A. (2009). Imaging circulating cells and lymphoid tissues with iron oxide nanoparticles. ASH Education Program Book, 2009(1), 720-726.

Gurunathan, S., Park, J. H., Han, J. W., \& Kim, J. H. (2015). Comparative assessment of the apoptotic potential of silver nanoparticles synthesized by Bacillus tequilensis and Calocybe indica in MDA-MB231 human breast cancer cells: targeting p53 for anticancer therapy. International Journal of Nanomedicine, 10, 4203.

Huang, D., Liao, F., Molesa, S., Redinger, D., \& Subramanian, V. (2003). Plastic-compatible low resistance printable gold nanoparticle conductors for flexible electronics. Journal of the Electrochemical Society, 150(7), G412.

Jadzinsky, P. D., Calero, G., Ackerson, C. J., Bushnell, D. A., \& Kornberg, R. D. (2007). Structure of a thiol monolayer-protected gold nanoparticle at 1.1 A resolution. science, 318(5849), 430-433.

Keat, C. L., Aziz, A., Eid, A. M., \& Elmarzugi, N. A. (2015). Biosynthesis of nanoparticles and silver nanoparticles. Bioresources and Bioprocessing, 2(1), 1-11.

Kelly, K. L., Coronado, E., Zhao, L. L., \& Schatz, G. C. (2003). The optical properties of metal nanoparticles: the influence of size, shape, and dielectric environment.

Mody, V. V., Siwale, R., Singh, A., \& Mody, H. R. (2010). Introduction to metallic nanoparticles. Journal of Pharmacy and Bioallied Sciences, 2(4), 282.

Zur Mühlen, A., Zur Mühlen, E., Niehus, H., \& Mehnert, W. (1996). Atomic force microscopy studies of solid lipid nanoparticles. Pharmaceutical Research, 13(9), 1411-1416.

Pandey, P., \& Dahiya, M. (2016). A brief review on inorganic nanoparticles. J Crit Rev, 3(3), 18-26.

Peng, X. H., Qian, X., Mao, H., \& Wang, A. Y. (2008). Targeted magnetic iron oxide nanoparticles for tumor imaging and therapy. International Journal of Nanomedicine, 3(3), 311.

Stuchinskaya, T., Moreno, M., Cook, M. J., Edwards, D. R., \& Russell, D. A. (2011). Targeted photodynamic therapy of breast cancer cells using antibody-phthalocyanine-gold nanoparticle conjugates. Photochemical \& Photobiological Sciences, 10(5), 822-831.

Laurent, S., Bridot, J. L., Elst, L. V., \& Muller, R. N. (2010). Magnetic iron oxide nanoparticles for biomedical applications. Future Medicinal Chemistry, 2(3), 427-449. 


\title{
Ocimum sanctum L., Phytochemistry and Effect on Cancer Cells
}

\author{
Ranjan Shaw, Bidisha Sarkar, Rakesh Mondol, Purbasha Sadhukhan, Md. Ahmed Karim, Kousik \\ Ghosh* \\ Department of Botany, Surendranath College, Kolkata, India \\ ${ }^{*}$ Corresponding Author's Email: kgbotsnc@gmail.com
}

\begin{abstract}
A well-known sacred plant known as tulsi or basil (holy) is an aromatic plant growing both in cultivated and wild manner. Its scientific name is Ocimum sanctum $\mathrm{L}$. belonging to family Lamiaceae. It is grown in every household in India as well as in many Southeast Asian countries. In several ancient systems of medicine Ocimum sanctum L. has a wide number of applications as drug in cardiopathy, haemopathy, leucoderma, asthma, bronchitis etc. This review incorporates taxonomy of Ocimum sanctum L., its chemical constituents and effect on cancer cells.
\end{abstract}

Keywords: Ocimum sanctum L; Taxonomy; Phytochemistry; Cancer Cells

\section{INTRODUCTION}

Cancer is one of the major diseases worldwide that continues to cause high mortality in the world. At the present time different plants are under clinical evaluation to understand the phytochemistry to utilize them to treat tumor growth (Nair, Gunasegaran \& Joshi, 1982). Herbs prescribed for cancer therapy in Ayurveda not only provide complete cure but also reduce the side effects and complications associated with cancer (Balachandran \& Govindarajan, 2005). The one of the most effective medicinal herbs is O. sanctum L., also known as tulsi (Charles \& Simon, 1990). This is an Indian sacred herb which is a member of the LAMIACEAE family. Ocimum was first listed by Linnaeus. He listed five species, of which $O$. sanctum is also one. About 200 species of herbs and shrubs are listed in the Ocimum genus (Linnaeus, 1753).

\section{LITERATURE REVIEW}

\section{Taxonomy}

Kingdom: Plantae

Subkingdom: Tracheobionta

Superdivision: Spermatophyta

Division: Magnoliophyta

Class: Magnoliopsida Subclass: Asteridae

Order: Lamiales

Family: Lamiaceae

Genus: Ocimum L.

Species: O. sanctum L.

The climatic conditions for growth of $O$. sanctum in warmth environment. The typical characteristics of $O$. sanctum is square stem, opposite and decussate leaves with many gland dots. The flowers of tulsi shows zygomorphic character and the feature of having bilabiate corolla. On distinct study $O$. sanctum has shown many therapeutic properties for which it is used to give relief from asthma, diabetes, infertility, and stress (Gupta, Prakash \& Srivastava, 2002). It is also used for the treatment of cancer, any 
disorder of nervous system which may leads to convulsions, repeated vomiting. Studies show that person having gastrointestinal, or any disorder of liver could be given decoction of $O$. sanctum leaves (Rajeshwari, 1992; Samudralwar \& Garg, 1996).

\section{Phytochemicals}

The following Phytochemicals are present in the leaves of $O$. sanctum-

1. Volatile Oil- eugenol, methyl eugenol, carvacrol and sesquiterpine hydrocarbon caryophyllene.

Ursolic acid has also been isolated from the O. sanctum leaves. Apart from ursolic acid, Nair et al., (2000) also isolated apigenin, luteolin, apigenin-7-0-glucuronide, luteolin-7-0-glucuronide, orientin, molludistin (Balanehru \& Nagarajan, 1991).

2. Flavonoids- orientin and vicenin.

These two flavonoids, obtained from the aqueous leaf extract of $O$. sanctum.

3. Phenolic compounds- cirsilineol, cirsimaritin, isothy-musin, isothymonin, apigenin, rosmarinic acid and appreciable quantities of eugenol (Kelm et al., 2000).

These phytochemicals are found in the fresh leaves and stems of $O$. sanctum

4. The leaves of $O$. sanctum are also known to contain traces of zinc, manganese, and sodium.

5. Seeds of $O$. sanctum possess the fatty oil (17.82\%) consisting of $6.9 \%$ palmitic acid, $2.1 \%$ stark acid, $15.7 \%$ linolenic acid, $66.1 \%$ linoleic acid and $9 \%$ oleic acid.

The oil content of seeds may vary depending on the geographical source.

Norr \& Wagner (1992) identified vicenin-2, rosmarinic acid, galuteolin, cirsilineol gallic acid, gallic acid methylester, gallic acid ethylester, protocatechic acid, vanillic acid, 4-hydroxybenzoic acid, vanillin, 4hydroxybenz-aldehyde, caffeic acid, chlorogenic acid from the ethanolic extract of $O$. sanctum. They also detected 2-phenyl-propaneglucoside I and II. The unsaponifiable matter yielded a small quantity of sitosterol. Three insoluble bromoglycerides were crystallized on direct bromination of the oil in dry ether (two dilinoleno-linolins melting at $157^{\circ} \mathrm{C}$ and $145^{\circ} \mathrm{C}$ respectively and a linolenodilinolin melting at $80^{\circ} \mathrm{C}$.

\section{Effect on cancer cells}

\section{i. Oral squamous cell carcinoma (OSCC)}

The leaves of $O$. sanctum showed effectiveness both as dry and aqueous extract. It acts as a cytologically toxic agents in the oral squamous cell which have become cancerous (Ca9-22). Studies have shown that anti cancerous phytochemicals of $O$. sanctum are more effective when aqueous extract is used. The most effective dose, HPC was $30 \mathrm{mg} / \mathrm{L}$ for aqueous extract of leaves and $35 \mathrm{mg} / \mathrm{L}$ for dry extract of leaves (Devi, Bisht, \& Vinitha, 1998).

\section{ii. Lung alveolar pulmonary adenocarcinoma}

The beneficial use and the role of ethanol extract of $O$. sanctum (EEOS) in the lung adenocarcinoma, investigation was done in the in vitro A549 cell culture model system by the treatment of A549 cells with $O$. sanctum of different concentrations. The cells growth, survival, and communication depend on cells adhesion to the extracellular matrix. Detachment adhesion assay was performed to analyze the ability of EEOS-inducing A549 cells (Singh, Majumdar \& Yadav, 1996).

\section{DISCUSSION}

While evaluating the role of phytochemistry of Ocimum sanctum on cancerous cells of various body parts as discussed above, it has been observed that depending upon the body parts extract of Ocimum sanctum acts as a powerful anti-carcinogenic agent Ocimum sanctum (Nadkarni \& Patwardhan, 1952; Nahak, Mishra \& Sahu, 2011). In case of cancerous cells of oral regions, the active phytochemicals show effective anti-cancer activity, acting as a chemotherapeutic agent. 
In lung alveolar pulmonary adenocarcinoma, Ethanol Extract of Ocimum sanctum has shown anticancerous activity by reducing proliferation of cells and its viability and increasing apoptosis in A 549 cells (Nörr \& Wagner, 1992).

Similar mode of action is observed in non-small cell lung carcinoma cell with an additional property of alteration of membrane potential of mitochondria.

\section{CONCLUSION}

Our results summarize that Ocimum sanctum extract effective for the treatment of oral cancer cells, lung alveolar pulmonary adenocarcinoma.

\section{ACKNOWLEDGEMENT}

The authors would like to acknowledge Department of Biotechnology, Government of India for the funding from DBT-STAR College grant, under which this review project was conducted. They are also grateful to the Principal and the DBT-STAR Coordinator for their support and encouragement in implementing this review project.

\section{REFERENCES}

Balachandran, P., \& Govindarajan, R. (2005). Cancer-an ayurvedic perspective. Pharmacological research, 51(1), 19-30.

Balanehru, S., \& Nagarajan, B. (1991). Protective effect of oleanolic acid and ursolic acid against lipid peroxidation. Biochemistry international, 24(5), 981-990.

Charles, D. J., \& Simon, J. E. (1990). Comparison of extraction methods for the rapid determination of essential oil content and composition of basil. Journal of the American Society for Horticultural Science, 115(3), 458-462.

Devi, P. U., Bisht, K. S., \& Vinitha, M. (1998). A comparative study of radioprotection by Ocimum flavonoids and synthetic aminothiol protectors in the mouse. The British Journal of Radiology, 71(847), 782-784.

Gupta, S. K., Prakash, J., \& Srivastava, S. (2002). Validation of traditional claim of Tulsi, Ocimum sanctum Linn. as a medicinal plant.

Kelm, M. A., Nair, M. G., Strasburg, G. M., \& DeWitt, D. L. (2000). Antioxidant and cyclooxygenase inhibitory phenolic compounds from Ocimum sanctum Linn. Phytomedicine, 7(1), 7-13.

Linnaeus, C. (1753). Species plantarum, ed. 1. Holmiae [Stockholm]: Impensis Laurentii Salvii.

Nadkarni, G. B., \& Patwardhan, V. A. (1952). Fatty oil from the seeds of Ocimum sanctum Linn (Tulsi). Current Science, 21(3), 68-69.

Nahak, G., Mishra, R. C., \& Sahu, R. K. (2011). Taxonomic distribution, medicinal properties and drug development potentiality of Ocimum (Tulsi). Drug Invention Today, 3(6).

Nair, A. R., Gunasegaran, R., \& Joshi, B. S. (1982). Chemical investigation of certain South Indian plants. Indian Journal of Chemistry Section B-organic Chemistry Including Medicinal Chemistry, 21(10), 979-980.

Nörr, H., \& Wagner, H. (1992). New constituents from Ocimum sanctum. Planta Medica, 58(06), 574574.

Rajeshwari, S. (1992). Ocimum sanctum. The Indian home remedy. Current Medical Scene. March-April (published by, Cipla Ltd., Bombay Central, Bombay).

Samudralwar, D. L., \& Garg, A. N. (1996). Minor and trace elemental determination in the Indian herbal and other medicinal preparations. Biological trace element research, 54(2), 113-121.

Singh, S., Majumdar, D. K., \& Yadav, M. R. (1996). Chemical and pharmacological studies on fixed oil of Ocimum sanctum. Indian journal of experimental biology, 34(12), 1212-1215. 


\title{
Induced Pluripotent Stem Cells (iPSCs): A Highway Under Construction
}

Kajal Das, Shreya Deb, Sagar Kumar Saha, Subhadipa Majumder*

Department of Zoology, Surendranath College, Kolkata, India

*Corresponding Author's Email: subhadipa@gmail.com

\begin{abstract}
Induced pluripotent stem cells are adult cells which are genetically reprogrammed and modified to embryonic stem cell like state. This cells can forcefully express genes which are essential for maintaining the embryonic stem cell properties. Reprogramming may be done by adding number of different transcription factors or application of miRNA and/or some small molecules. The advantages of iPSCs are, iPSCs can be formed from the tissue of the same patient who will receive the transplantation; thus, the patients will avoid immune rejection. Ethical issues also can be evaded because adult cells are harvested from a willing person without harming him/her. iPSC permits the development of infinite source of any type of human cell of interest which are needed for therapeutic purposes.
\end{abstract}

Keywords: Stem Cell; iPSCs; Reprogramming

\section{INTRODUCTION}

Stem cells has ability of self-renewal and unlimited proliferation. Stem cells have three main properties: they can divide and renewing themselves for long time; they are unspecialized; and can form specialized cell types. There are several types of stem cells (embryonic stem cells or ESCs, fetal stem cell, non-embryonic or adult stem cells, induced pluripotent stem cells or iPSCs and cord blood stem cells and amniotic fluid stem cells, Figure 1 and 2) which can be used for different purposes like research, therapeutic etc. The induced pluripotent stem cells allow simple adult somatic cells to be modified and reprogrammed into stem cell like state and can be used as naive stem cells.

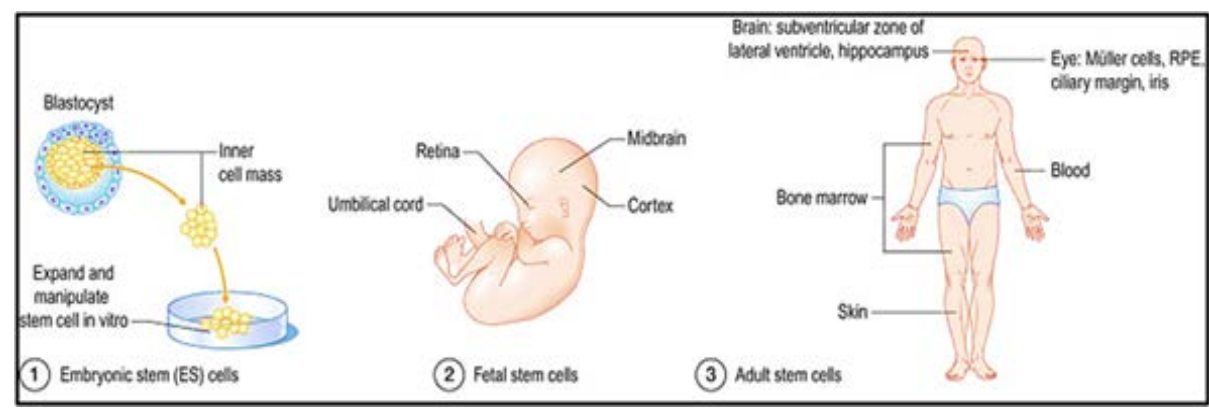

Figure 1: Types of Stem Cells (Source: Snyder \& Yip, 2011)

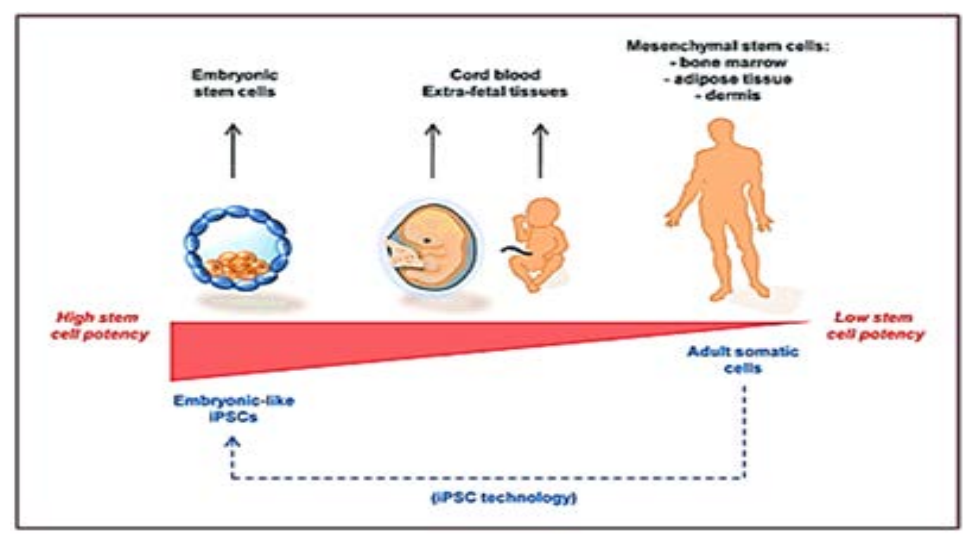

Figure 2: Sources of Stem Cells (Source: Duscher et al., 2015) 


\section{LITERATURE REVIEW AND DISCUSSION}

\section{Stem Cell Division Based on Potency}

In development, cell fate and potency are gradually constrained (from totipotent to unipotent) until a final differentiation of cell. Life begins from the zygote, a single cell. The zygote and early stages of blastomeres are totipotent which can develop a whole organism. But the developmental potential of blastomeres progressively drops (by a succession of inductive signals) (Figure 3).

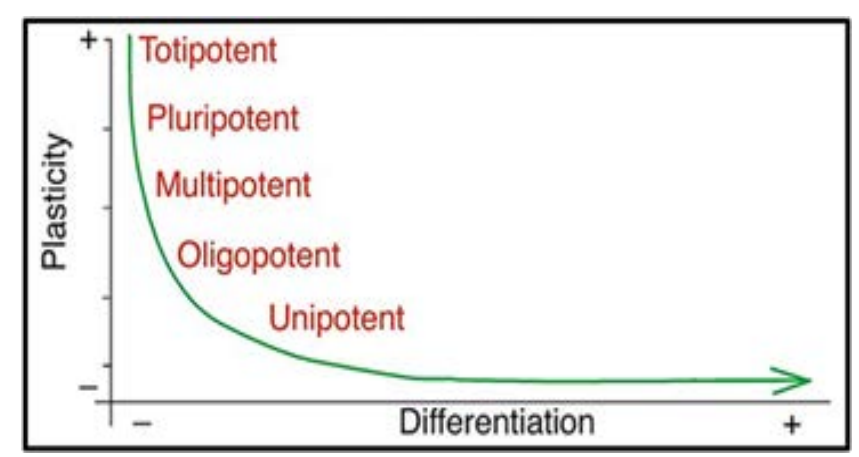

Figure 3: Cell Potency Decreases during Development (Source: Trucco, 2005)

\section{Stem Cells According to Their Developmental Potential}

Totipotent Cell: (Lat. totipotentia, "ability for all ") A cell that can form all types of cells in an organism. It can also form the extra-embryonic structures of the organism. (Figure 4). Example: Zygote, early stages of blastomeres (4-cell stage) of an embryo.

Pluripotent Cell: A cell that that can give rise all tissues of an organism (endoderm, mesoderm or ectoderm) except extra-embryonic structures (Figure 1.4). Example: Inner cell mass (ICM) cells.

Types of Pluripotent Cell:

- Embryonic stem cells

- Induced pluripotent stem cells

The zygote, the single cell from which the embryo is derived, is a totipotent cell. Later, the inner mass cells of the zygote can differentiate into many types of tissues of the organism. Inner mass cells are the source of pluripotent stem cell.

Multipotent Cell: Cells that develop into more than one cell type but not all. Cell fate is restricted (Figure 5). Example: Adult hematopoietic stem cells. It can form multiple types of blood cells, but certainly not a muscle cell.

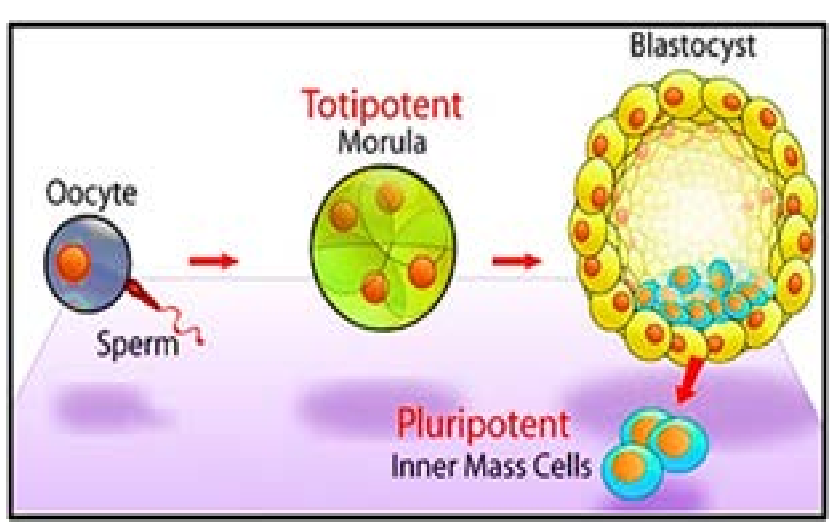

Figure 4: Totipotent and Pluripotent Stem Cell (Source: ScienceDebate, 2011)

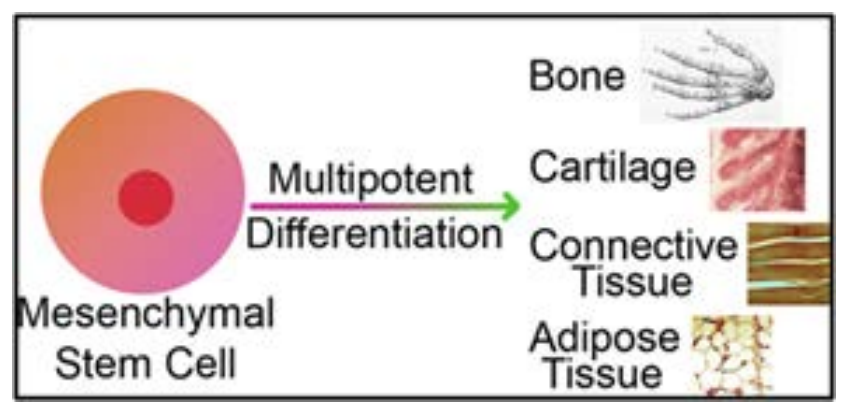

Figure 5: Multipotent Stem Cell (Source: Rowland, 2009)

Mesenchymal stem cells can form wide variety of cells.

Oligopotent Cell: This cells can differentiate into a limited cell types (Figure 6). Example: A lymphoid stem cell can form various blood cells such as $B$ and T cells but not a red blood cell. 


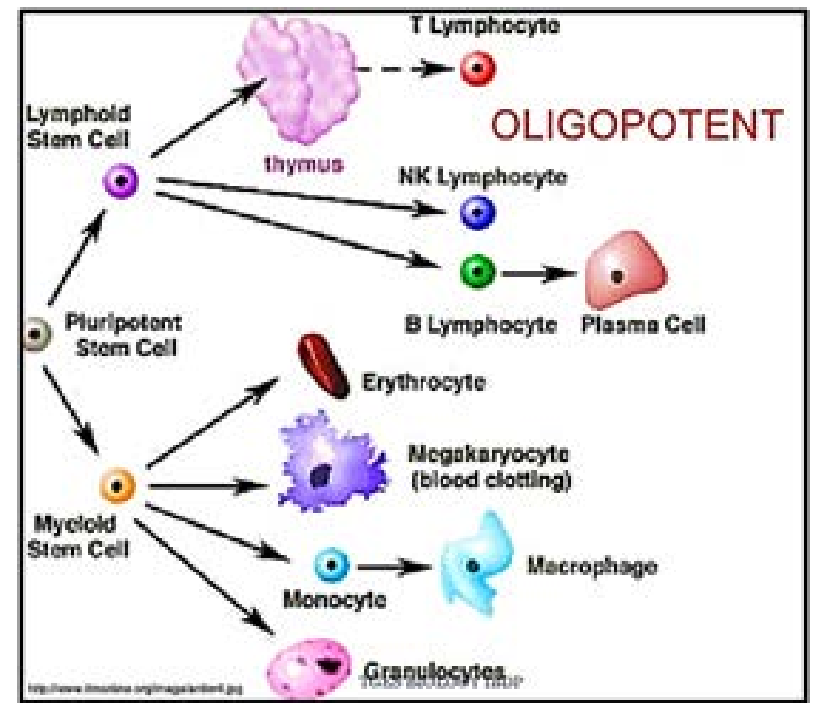

Figure 6: Oligopotent Stem Cell (Source: Slideshare, 2011)

Lymphoid cells can differentiate into different lymphocytes.

Unipotent Cell: Unipotent cells can only copy of it (Figure 7). Example: Epithelial cells self-renew all over its adult life.

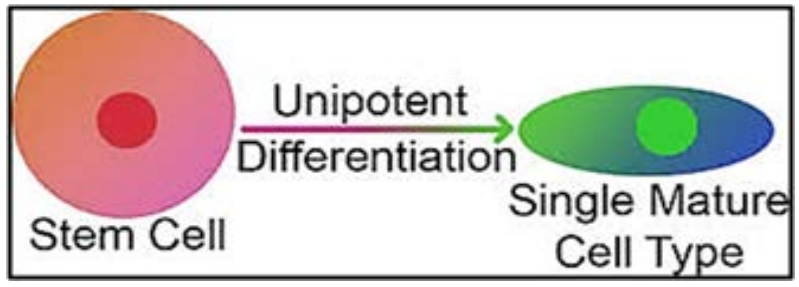

Figure 7: Unipotent Stem Cell Can Form a Single Type of Cell of the Organism (Source: Rowland, 2009)

\section{Induced Pluripotent Stem Cells (iPSCs)}

iPSCs can be artificially acquired by reprogramming animal cells by inducing some factors which leads to "forced" expression of genes. iPSC can be formed from skin or blood cells. These cells are reprogrammed to go back into embryonic-like pluripotent state that help to develop an infinite source of different type of cell needed for various purposes. Adult mammalian cells can be reprogrammed to a pluripotent state by adding of few embryonic transcription factors. This factors stimulate and modulate the potential of cells during reprogramming process (Figure 8).

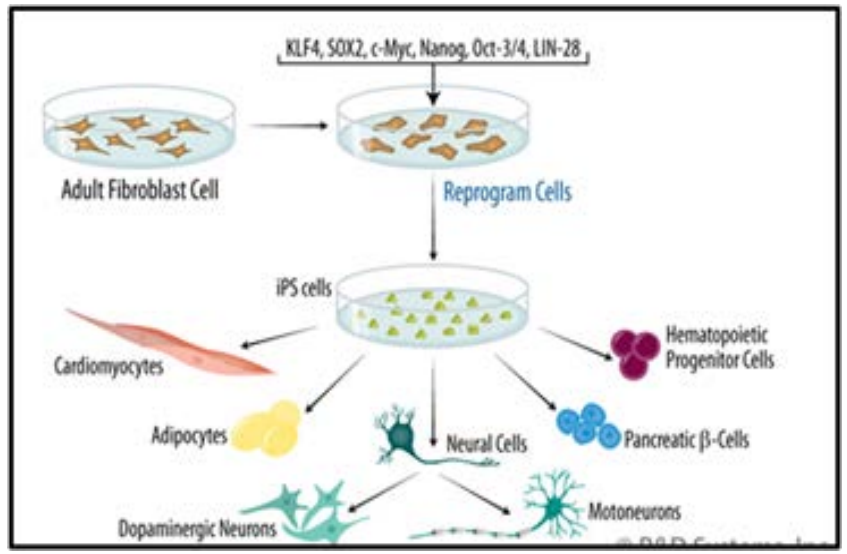

Figure 8: Reprogramming of adult cell to generate iPSCs (Source: R\&Dsystems) 
Adult somatic cells are cultured in vitro and stimulated with expression vectors which encodes the necessary transcription factors related with pluripotency.

\section{Steps for Reprogramming of Somatic Cells}

Oct4, Sox2, KIf4, and c-Myc transcription factors are used to reprogram the adult cells. Reprogramming may be attained by adding different mixture of transcription factors, application of miRNA and some small molecules (Valproic acid, Sodium butyrate, Vitamin C etc.). Various direct delivery methods are used such as using synthetic mRNA etc. (Figure 9).

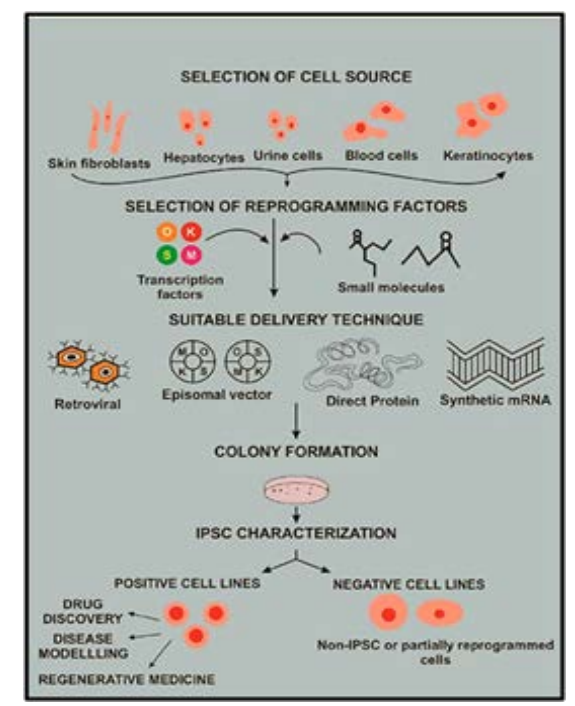

Figure 9: Steps for Reprogramming (Source: Singh et al., 2015a)

Suitable cell sources are selected and stimulated by pluripotent cocktail (OSKML) with compatible delivery method. Expression of reprogramming factors prevent the expression of the genes which are responsible for cell differentiation and activate the genes for pluripotency maintenance.

\section{Types of Donor Cells for iPSC Generation}

Induced Pluripotent Stem Cells are derived from skin or blood cells that have been reprogrammed into a pluripotent state for therapeutic purposes (Table 1).

Human hair follicles are used for iPSCs generation (Higgins et al., 2012). Aasen et al. (2008) established the use of keratinocytes. Blood is considered the appropriate source for iPSCs. Recently, renal epithelial cells are used for the formation of iPSCs (Zhou et al., 2011).

Table 1: Cell Source Types and Necessary Reprogramming Factors (Source: Singh et al., 2015b)

\begin{tabular}{|l|l|l|}
\hline \multirow{2}{*}{ Cell types } & $\begin{array}{l}\text { Reprogramining } \\
\text { factors }\end{array}$ & Reprogrammiag efficieacy (approximate) \\
\hline \multirow{2}{*}{ Fiteoblasts } & OSKM & $0.02 \%$ \\
\cline { 2 - 3 } & OSLN & $0.02 \%$ \\
\hline Keratinocytes & OSKM & ND \\
\hline Cord blood endothelial cells & OSLN & $<0.01 \%$ \\
\hline Cord blood stem cells & OSKM & $0.01 \%$ \\
\hline Neural stem cells & O & $<0.004 \%$ \\
\hline Melanocytes & OSKM & $0.19 \%$ \\
\hline Amriotic cells & OSKM & $0.05-1.5 \%$ \\
\hline Adipose derived stem cells & OSKM & $0.50 \%$ \\
\hline Hepatocytes & OSKM & $0.10 \%$ \\
\hline Circulating T cells & OSKM & $0.10 \%$ \\
\hline Astrocytes & OSKM & $>0.02 \%$ \\
\hline Peripheral blood & OSKM & $0.01 \%$ \\
\hline Kidney mesangial cells & OSKM & $\mathrm{ND}$ \\
\hline Urise cells & OS & $4 \%$ \\
\hline
\end{tabular}


Selection of Reprogramming Factors (O-Oct4; S-Sox2; K-KIf4; M- c-Myc; N-Nanog; L-Lin28)

OSKM (Oct4, Sox2, KIf4, c-Myc) cocktail was used by Yamanaka's group extensively by researchers on a for human somatic cells (Aasen et al., 2008). Thomson's group reported another combination of factors that were Oct 3/4, Sox2, Nanog, and Lin 28 (OSNL). But the OSKM and OSNL cocktails showed low-efficiency rate (Zhou, 2011) (Figure 10).

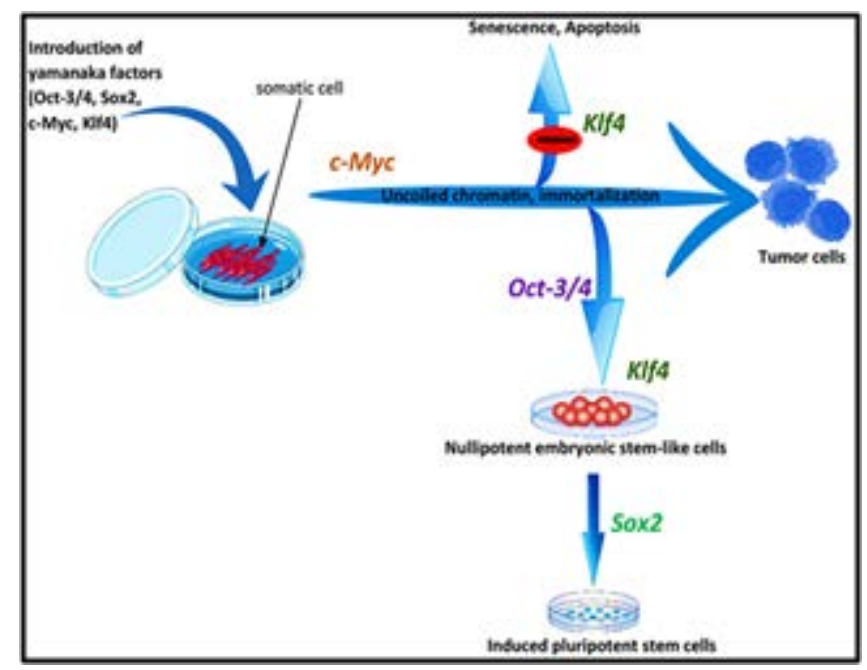

Figure 10: OSKM Factors in iPSCs Induction (Source: Yamanaka, 2012)

\section{Transcription Factors and Non-Coding RNAs Used During iPSC Induction}

Most common factors responsible for maintenance of pluripotency are: Oct4, Sox2, KIf4, c- Myc, and Nanog.

Oct-3/4 and Sox1, Sox2, Sox3, and Sox15 are essential transcriptional regulators involved in the induction. Certain members of the KIf and Myc family (c-myc, L-myc, and N-myc), LIN28 and Nanog reported with high induction efficiency. Small molecules, microRNA's (miR) and culturing conditions also can enhance the iPSC quality and efficiency of reprogramming (Table 2).

Table 2: Types of Reprogramming Factors. Upregulated (+); Downregulated (-) (Source: Brouwer, Zhou \& Kasri, 2016)

\begin{tabular}{|c|c|c|c|}
\hline Aropuming bitin & Fantis & Aficow pationg & 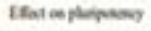 \\
\hline Oat & 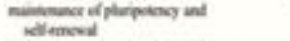 & 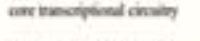 & * \\
\hline $\sin 2$ & 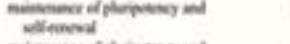 & 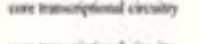 & * \\
\hline sai & 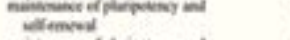 & 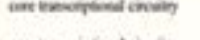 & * \\
\hline 6 atp & 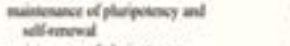 & 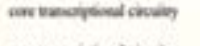 & * \\
\hline Las & 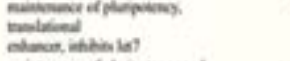 & 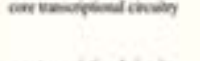 & * \\
\hline Nent & 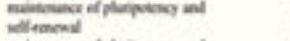 & 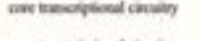 & * \\
\hline seat & 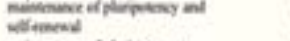 & 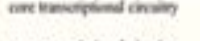 & * \\
\hline in & 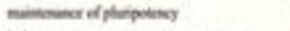 & 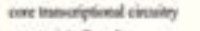 & * \\
\hline$\infty 0$ & 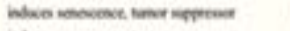 & nophented ople & - \\
\hline N & 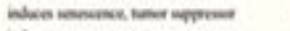 & aproniat eqde & - \\
\hline risowe & 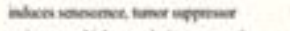 & aptribied ople & - \\
\hline cuss & 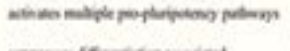 & 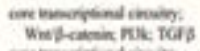 & * \\
\hline Later & 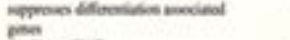 & 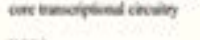 & * \\
\hline tora & Falices IVI & $\operatorname{ratb}$ & * \\
\hline Maso & politholer & poposiniall opde & * \\
\hline mase & pos inther & 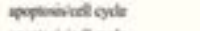 & - \\
\hline Cycla bi & 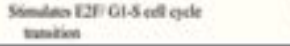 & 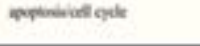 & * \\
\hline
\end{tabular}

\section{Small Molecules Used During iPSC Induction}

Yamanaka factors are used for somatic cells reprogramming by many researchers, but this factors have lower reprogramming efficiency. Valproic acid, CHiR99021, sodium butyrate, vitamin C, parnate, 
5-Azacytidine, RG108 etc. now can replace the Yamanaka factors (Table 3 and 4).

Table 3: Roll of Small Molecules in Reprogramming (Source: Singh et al., 2015b)

\begin{tabular}{|l|l|}
\hline Compound & Mechanism of action \\
\hline Valproic acid & Inhibits of histone deactlyase \\
\hline CHiR99021 & Inhibits of glycogen synthase kinase 3 \\
\hline Sodium butyrate & Inhibits of histone deacetylase \\
\hline Vitamin C & Lowers reactive oxygen species \\
\hline Pamate & Inhibits of histone demethylase \\
\hline 5-Azacytidine & Inhibits of DNA methyltransferase \\
\hline RG108 & Blocks DNA methyltransferase \\
\hline
\end{tabular}

Table 4: Small Molecules and Related Pathways (Source: Brouwer, Zhou \& Kasri, 2016)

\begin{tabular}{|c|c|c|c|}
\hline Reprogramming factors & Function & Affected pathway & Effect on pluripotency \\
\hline Vitamin C & alleviates cell senescence/antioxidant & Hypoxia response & + \\
\hline Valproic acid & inhibits histone deacetylases & Chromatin remodeling & + \\
\hline CHIR99021 & GSK3-inhibitor & PI3k; Wnt/ß-catenin & + \\
\hline Pamate & lysine-specific demethylase 1 inhibitor & Chromatin remodeling & + \\
\hline SB431542 & ALK5/TGF $\beta$ receptor inhibitor & TGF $\beta$ & + \\
\hline PD0325901 & MEK inhibitor & MAPK/ERK & + \\
\hline BLX-01294 & Methyltransferase G9a inhibitor & Chromatin remodeling & + \\
\hline Lithium & GSK3-inhibitor & PI3k; Wnt/B-catenin & + \\
\hline Maxadilan & $\begin{array}{l}\text { downregulates Caspase3 and 9, } \\
\text { anti-apoptotic }\end{array}$ & apoptosis & + \\
\hline 8-Br-cAMP & Protein kinase $\mathrm{A}$ activator & CAMP & + \\
\hline$A-83-01$ & ALK5/TGF $\beta$ receptor inhibitor & TGF $\beta$ & + \\
\hline Trazovivin & promotes survival, ROCK inhibitor & PI3k & + \\
\hline Y-27632 & promotes survival, ROCK inhibitor & PI3k & + \\
\hline EPZ004777 & DOT1L inhibitor & Chromatin remodeling & + \\
\hline DAPT & $\begin{array}{l}\text { Inhibits Notch/ increases core transcription } \\
\text { factor expression/inhibits p53 pathway }\end{array}$ & $\begin{array}{l}\text { core transcriptional } \\
\text { circuitry/ apoptosis }\end{array}$ & + \\
\hline
\end{tabular}

\section{Other Techniques to Reprogram Somatic}

There are various ways for achieving pluripotency other than introducing the transcription factors and small molecules. The common methods are Somatic Cell Nuclear Transfer (SCNT) and Cell fusion.

Nuclear Transfer: Nuclear reprogramming was first achieved by SCNT. The adult somatic cell nucleus was removed and transferred to an enucleate oocyte which was unfertilized (Figure 11). In 1996, first cloned sheep was developed by nucleus transfer of blasomere from sheep embryo to unfertilized and enucleated egg (Campbell et al., 1996). Different cells such as hepatocytes, leukocytes, neuronal cells, lymphocytes were also used (Hochedlinger \& Jaenisch, 2003).

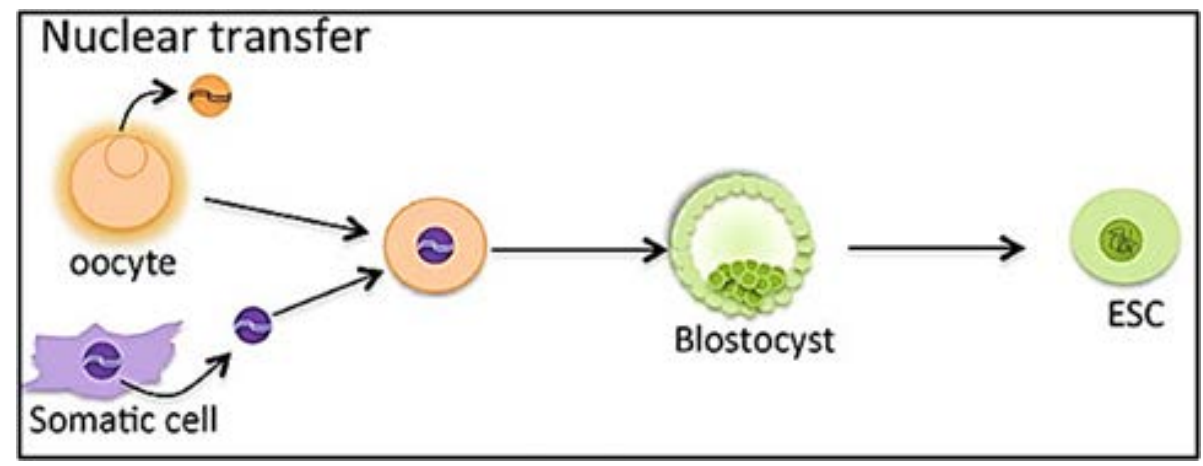

Figure 11: SCNT Technique to Produce Pluripotent Stem Cell Formation (Source: Chen et al., 2014)

Cell Fusion: In this procedure the pluripotent cells are fused with somatic cells (Figure 12). Over expression of embryonic transcription factors dominate the adult cell properties and maintain the 
pluripotency (Tada et al., 2001; Pralong, Trounson \& Verma, 2006).

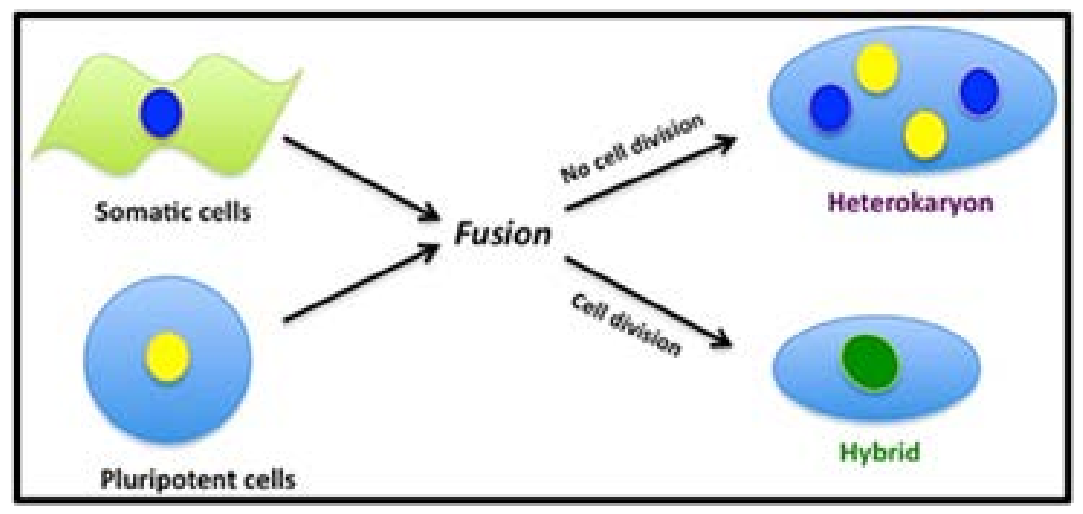

Figure 12: Cell Fusion Technique to Produce iPSCs (Source: Nigro \& Verfaillie, 2013)

Stressor: When cells from brain, skin, lung, and liver were exposed to stress and low $\mathrm{pH}$, some cells was survived from stress and could be reprogrammed to multipotent stem cells. This is called stimulustriggered acquisition of pluripotency (STAP; Figure 13). The procedure had the advantage that the cells were reprogrammed without embryo destruction or entry of any exogenous genes. (Yamanaka 2012).

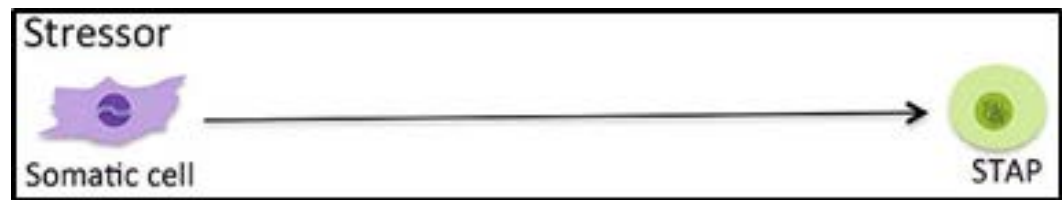

Figure 13: Generation of Pluripotenty by Stress. (Source: Chen et al., 2014)

Parthenogenesis: Using a series of chemical treatments, it's possible to trick an egg into developing into an embryo without being fertilized by sperm.

\section{Methods for iPSC Generation}

Varoious delivery methods have been introduced to reprogramme the somatic cells. It can be classified into two categories: integrative systems (integration of exogenous genetic material into the host genome) and non-integrative systems (no integration of genetic material into the host genome) (Figure 14).

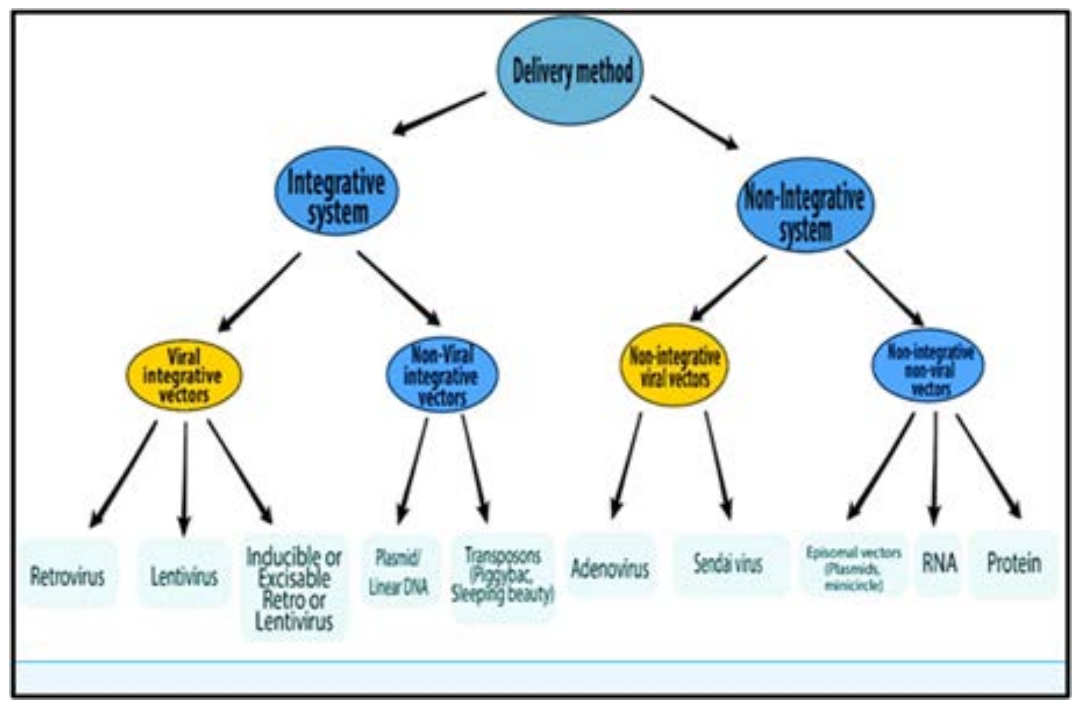

Figure 14: Various Delivery Methods of iPSC Induction (Source: Omole \& Fakoya, 2018) Integrative Method

Viral Vector-Based Delivery: MMLV-derived retroviral vectors are used for production of mouse and 
human iPSCs. Lentiviral vectors are also used for its higher cloning capacity.

Transposon Delivery: Delivery of OSKM by Piggyback (PB) transposon is another method. This delivery occurs with high efficiency, and this is the only one method that allows a precise deletion of the cassette.

\section{Non-Integrative Method}

Viral Vector-Based Delivery: Adenoviral vectors are used for making iPSCs. Adenoviral vectors can carry $36 \mathrm{~Kb}$ and can infect both dividing and non-dividing cells.

Protein/RNA Based Delivery: Some scientist fused OSKM proteins with poly-arginine in Escherichia coliand generated iPSCs.

\section{iPSC Cell Culture}

hiPSCs/human ESCs (hESCs) are generated on living feeder cells, such as mouse embryonic fibroblasts (MEFs) or SNL cells or on a feeder-free culture substrate such as Matrigel, fibronectin or human recombinant laminin-511.

\section{Feeder Cell Dependent Culture}

Adherent growth-arrested, viable and bioactive cells are the feeder layer cells. These cells are used as a substratum for cell growth. These cells release the growth factors which support the growth of target cells. Some feeder cells synthesize ligands or cytokines which are needed to support the cultured target cells (Roy et al., 2001).

In this system, the fibroblast-seeded plates are prepared. Feeder cells provide the growth factors and extracellular matrix proteins which create a support matrix for target cell attachment and proliferation (Figure 15).

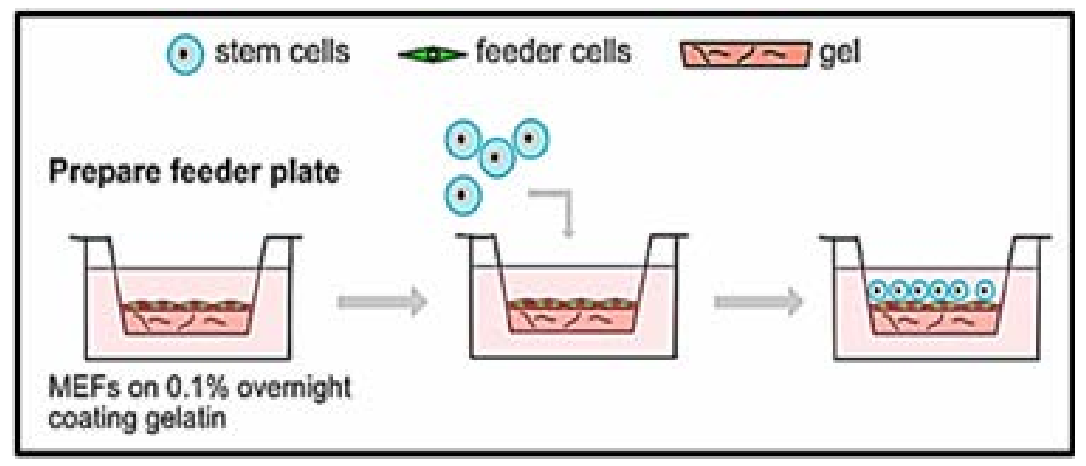

Figure 15: Feeder Cell Dependent Culture. (Source: Creative Bioarray)

\section{Feeder Cell Independent Culture}

In this method, extracellular matrix is used in the place of feeder cells. The feeder-free system is manipulated by adding the essential amino acids, salts, other nutritional elements, and growth factors in the media (Figure 16).

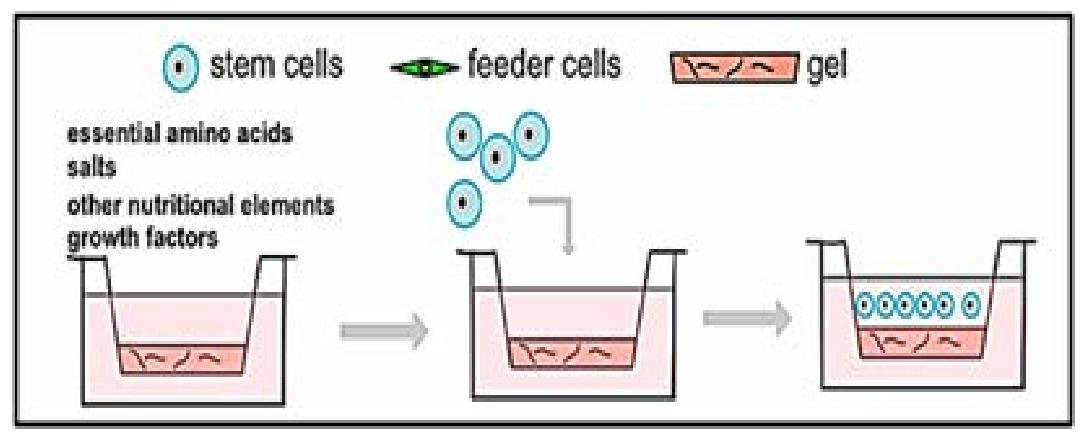

Figure 16: Feeder Cell Independent Culture (Source: Creative Bioarray) 


\section{Applications of iPSC}

The application of iPSCs is wide as it is used for the following mentioned approaches.

\section{Disease Modeling and Drug Development}

iPS cells have been generated for different human genetic diseases such as Down syndrome, liver disease and polycystic kidney disease (Figure 17). An international collaborated project, Stem BANCC has set their goal to generate a library of $1,500 \mathrm{iPS}$ cell lines which will be used in early drug testing.

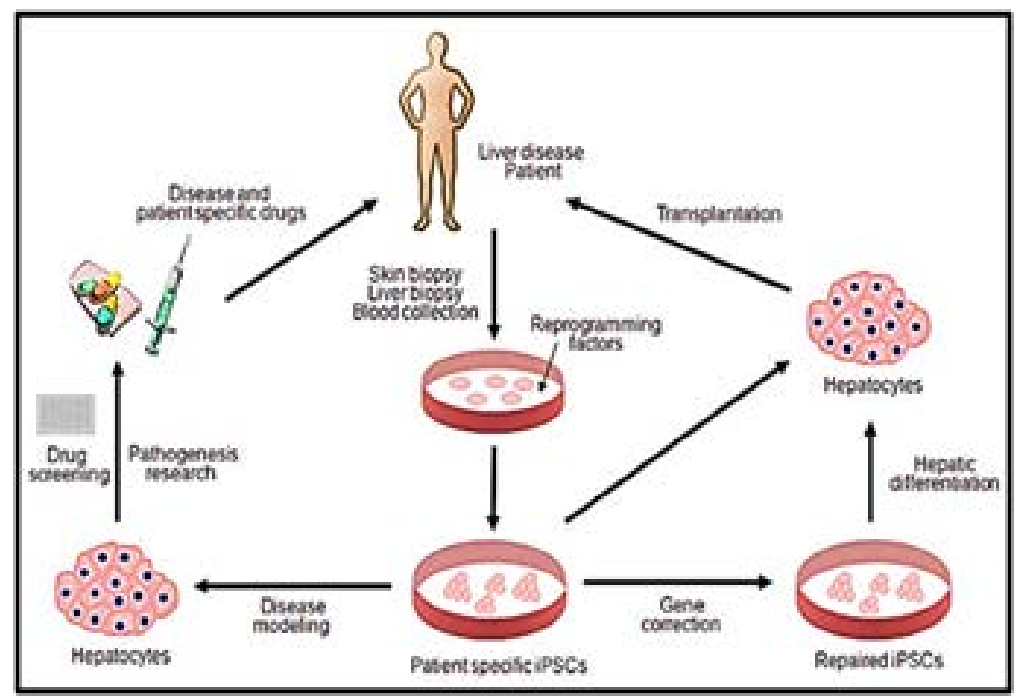

Figure 17: Applications of Human iPSCs for Liver Diseases (Source: Chun, Chaudhari \& Jang, 2010)

\section{Organ Synthesis}

Induced pluripotent stem cells (iPSCs) also help in generating patient-specific organs. Delivering autologous, and thus immune-matched, organs to replace a patient's own diseased organs help to eliminate the detrimental effects of immunosuppression (Figure 18). A study was reported from Japan where Human 'liver buds' (iPSC-LBs) were grown from a mixture of three different kinds of stem cells: hepatocytes (liver cells) coaxed from iPSCs; endothelial stem cells (lining of blood vessels) from umbilical cord blood; and mesenchymal stem cells (connective tissue).

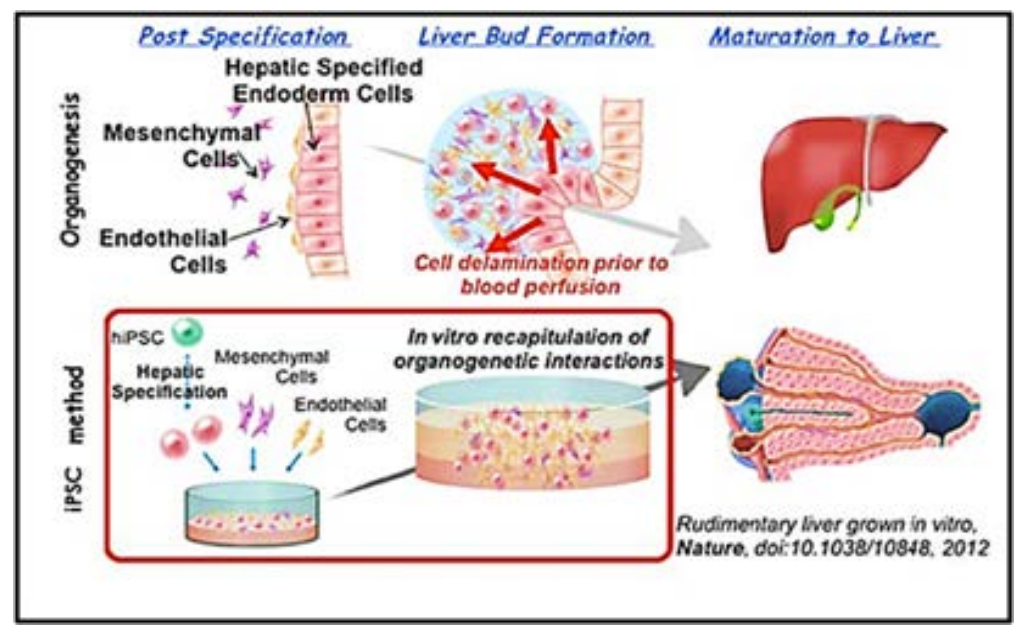

Figure 18: Formation of Liver Bud from Pluripotent Stem Cells (Source: Zhang et al., 2015)

\section{Tissue Repair}

In hydrogel, 3D skeletal myogenic differentiation of pluripotent cells was induced in different studies. 
Study showed that the artificial muscles recapitulated the features of skeletal muscle and could be implanted into immunodeficient mice (Maffioletti et al., 2018) (Figure 19).

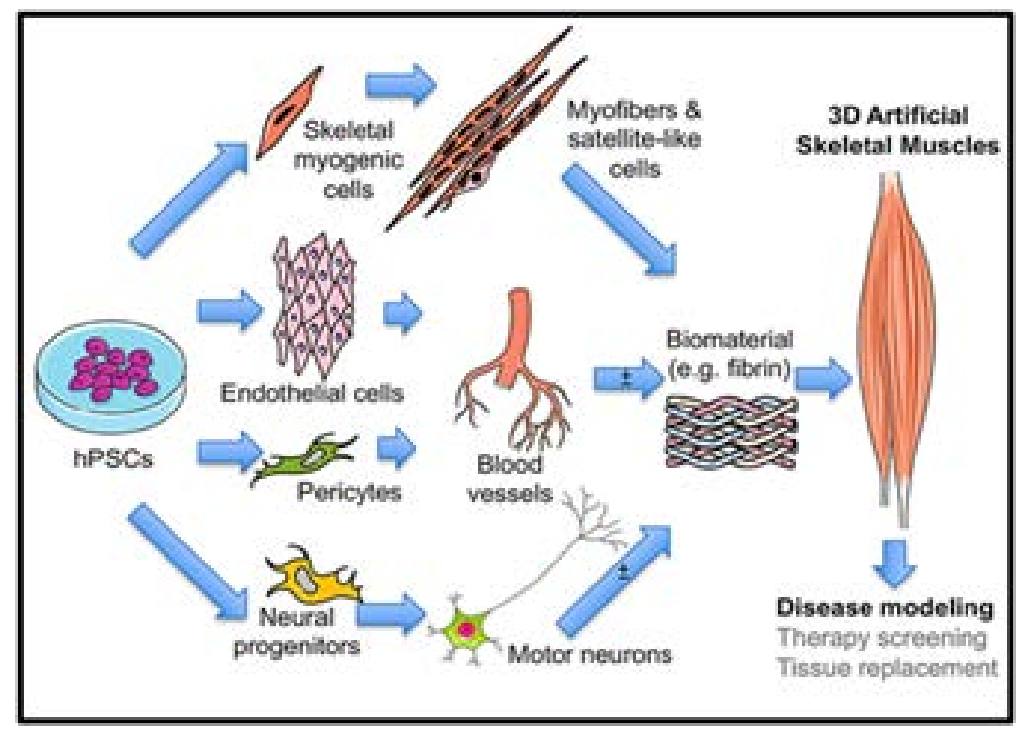

Figure 19: Skeletal Muscle Formation from iPSCc (Source: Maffioletti et al., 2018)

\section{Red Blood Cell Synthesis}

Type O red blood cells were synthesized in 2014 at the Scottish National Blood Transfusion Service by using iPSC. This type $O$ red blood cells can be transfused into all type of patients (Focosi and Pistello 2016) when it is necessary.

\section{CONCLUSION}

Induced pluripotent stem cells provide researchers and clinicians a $\mathrm{n}$ new line of patient-specific therapies. Reprogramming adult tissues has innumerable probable applications to drug development, research on stem cells and developmental processes. However, many technical and basic science issues are there for iPSC technology which can't be realized fully. For medicine applications, patient safety is the prime consideration for doctors. Furthermore, reprogramming is currently too inefficient for routine clinical practice. Thus, unscrambling the molecular mechanisms which direct the reprogramming is a major step which wide the path of this new approach.

\section{ACKNOWLEDGEMENT}

The authors express their sincere thanks to Department of Biotechnology, Government of India for the funding from DBT-STAR College grant, under which this review project was conducted. We are also grateful to the Principal and the DBT-STAR Coordinator, Surendranath College, for their support and encouragement in implementing this review project at undergraduate level.

\section{REFERENCES}

Aasen, T., Raya, A., Barrero, M. J., Garreta, E., Consiglio, A., Gonzalez, F., ... \& Belmonte, J. C. I. (2008). Efficient and rapid generation of induced pluripotent stem cells from human keratinocytes. Nature Biotechnology, 26(11), 1276-1284.

Brouwer, M., Zhou, H., \& Kasri, N. N. (2016). Choices for induction of pluripotency: recent developments in human induced pluripotent stem cell reprogramming strategies. Stem Cell Reviews and Reports, 12(1), 54-72.

Campbell, K. H., McWhir, J., Ritchie, W. A., \& Wilmut, I. (1996). Sheep cloned by nuclear transfer from a cultured cell line. Nature, 380(6569), 64-66. 
Chen, K.G., Mallon, B.S., McKay, R.D.G., \& Robey, P.G. (2014). Human Pluripotent Stem Cell Culture: Considerations for Maintenance, Expansion, and Therapeutics. Cell Stem Cell, 14(1), 13-26.

Chun, Y. S., Chaudhari, P., \& Jang, Y. Y. (2010). Applications of patient-specific induced pluripotent stem cells; focused on disease modeling, drug screening and therapeutic potentials for liver disease. International Journal of Biological Sciences, 6(7), 796.

Creative Bioarray. Stem Cell Culture Guide. https://www.creative-bioarray.com/support/stem-cellculture-guide.htm

Duscher, D., Barrera, J., Wong, V. W., Maan, Z. N., Whittam, A. J., Januszyk, M., \& Gurtner, G. C. (2016). Stem cells in wound healing: the future of regenerative medicine? A mini-review. Gerontology, 62(2), 216-225.

Focosi, D., \& Pistello, M. (2016). Effect of Induced Pluripotent Stem Cell Technology in Blood Banking. Stem Cells Translational Medicine, 5(3), 269-274.

Higgins, C. A., Itoh, M., Inoue, K., Richardson, G. D., Jahoda, C. A., \& Christiano, A. M. (2012). Reprogramming of human hair follicle dermal papilla cells into induced pluripotent stem cells. The Journal of Investigative Dermatology, 132(6), 1725.

Hochedlinger, K., \& Jaenisch, R. (2003). Nuclear transplantation, embryonic stem cells, and the potential for cell therapy. New England Journal of Medicine, 349(3), 275-286.

Maffioletti, S. M., Sarcar, S., Henderson, A. B., Mannhardt, I., Pinton, L., Moyle, L. A., ... \& Tedesco, F. S. (2018). Three-dimensional human iPSC-derived artificial skeletal muscles model muscular dystrophies and enable multilineage tissue engineering. Cell Reports, 23(3), 899-908.

Nigro, A.L., \& and Verfaillie, C.M. (2013). Multiple Paths to Reprogramming. In D. Bhartiya, \& N. Lenka (Ed.), Pluripotent Stem Cells.

Omole, A.E., \& Fakoya, A.O.J. (2018). Ten years of progress and promise of induced pluripotent stem cells: historical origins, characteristics, mechanisms, limitations, and potential applications. PeerJ, 6, e4370.

Pralong, D., Trounson, A. O., \& Verma, P. J. (2006). Cell fusion for reprogramming pluripotency. Stem Cell Reviews, 2(4), 331-340.

R\&Dsystems. Differentiation Potential of Induced Pluripotent Stem Cells. https://www.rndsystems. $\mathrm{com} /$ resources/articles/differentiation-potential-induced-pluripotent-stem-cells

Rowland, T. (2009). Visual Stem Cell Glossary. https://www.allthingsstemcell.com/glossary/

Roy, A., Krzykwa, E., Lemieux, R., \& Néron, S. (2001). Increased efficiency of gamma-irradiated versus mitomycin $\mathrm{C}$-treated feeder cells for the expansion of normal human cells in long-term cultures. Journal of Hematotherapy and Stem Cell Research, 10(6), 873-80.

ScienceDebate. (2011). What are Stem Cells? Embryonic and Adult Stem Cells. https://sciencedebate.com/science-blog/what-are-stem-cells-embryonic-and-adult-stem-cells

Singh, V. K., Kalsan, M., Kumar, N., Saini, A., \& Chandra, R. (2015a). Induced pluripotent stem cells: applications in regenerative medicine, disease modeling, and drug discovery. Frontiers in Cell and Developmental Biology, 3, 2.

Singh, V. K., Kumar, N., Kalsan, M., Saini, A., \& Chandra, R. (2015b). Mechanism of induction: induced pluripotent stem cells (iPSCs). Journal of Stem Cells, 10(1), 43.

Slideshare. (2011). Stemcells. https://www.slideshare.net/duthaman/stemcells

Snyder, E.Y., \& Yip, S. (2011). 7 - Stem Cell Biology. In Richard A. Polin, W. William, W. Fox, \& S.H. 
Abman (Ed.), Fetal and Neonatal Physiology ( $4^{\text {th }}$ ed.), Saunders.

Tada, M., Takahama, Y., Abe, K., Nakatsuji, N., \& Tada, T. (2001). Nuclear reprogramming of somatic cells by in vitro hybridization with ES cells. Current Biology, 11(19), 1553-1558.

Trucco, M. (2005). Regeneration of the pancreatic $\beta$ cell. The Journal of clinical investigation, 115(1), 5-12.

Yamanaka, S. (2012). Induced pluripotent stem cells: past, present, and future. Cell Stem Cell, 10(6), 678-684.

Zhang, R. R., Koike, H., \& Takebe, T. (2015). The Visualization of Human Organogenesis from Stem Cells by Recapitulating Multicellular Interactions. In Hyper Bio Assembler for 3D Cellular Systems (pp. 275-283). Springer, Tokyo.

Zhou, T., Benda, C., Duzinger, S., Huang, Y., Li, X., Li, Y., ... \& Esteban, M. A. (2011). Generation of induced pluripotent stem cells from urine. Journal of the American Society of Nephrology, 22(7), 1221-1228. 


\title{
Polycystic Ovarian Syndrome Thieving Womanhood
}

\author{
Arun Jana, Sumona Naskar, Subhadra Roy* \\ Department of Chemistry, Surendranath College, Kolkata, India \\ ${ }^{*}$ Corresponding Author's Email: roysubhadra40@gmail.com
}

\begin{abstract}
Polycystic ovarian syndrome (PCOS) is one of the most important and common cause of anovulatory infertility. This gynecological problem affects a large proportion of women of the reproductive age and shows multiple symptoms. It results in diseases like hyperandrogenism, obesity, anovulation, cancer etc.Different factors could result in different manifestations and many of these are related to predispositions. Exact symptoms and manifestations are needed to be identified for the exact diagnosis of PCOS, especially among the adolescent patients because there is much similarity between features of PCO syndrome and physiological findings in puberty. The most common signs of this disease are infrequent or prolonged menstrual periods or excess male hormone levels or development of numerous small collections of fluid associated with failure in regular egg release. The excess androgen levels may also cause more facial and body hair growth (hirsutism), insulin resistance and acne. The exact cause of PCOS is not known. However, genetic reasons and excess androgen production from the ovaries may play a role in PCOS development. The major diagnostic methods include visual examination of the pelvic region, blood tests for measuring glucose tolerance, fasting cholesterol and triglyceride levels. Ultrasonography can help to diagnose PCOSby detecting small antral follicles which synthesize serum $\mathrm{AMH}$. Early diagnosis and treatment with proper medication and lifestyle changes can surely help to manage the disease.
\end{abstract}

\section{Keywords: PCOS; Hyperandrogenism; Infertility; Womanhood}

\section{INTRODUCTION}

Polycystic ovarian syndrome is an alarming health condition that mostly affects women of the reproductive age and its prevalence is about $10 \%$ (Varghese, Thomas \& Sindhuja, 2015). Changes in lifestyle with increasing modernization had increased the diagnosis of PCOS in young girls. According to Rosenfield, many factors like overweight mother, low birth weight of baby, obesity, precocious puberty, metabolic syndrome and many other factors have increased the risk of polycystic ovarian syndrome. Evidences suggest that PCOS in human beings can be initiated due to prenatal androgen exposure which is mainly caused due to congenital adrenal hyperplasia. Also, a high level of luteinizing hormone and dehydroepiandrosterone sulfate levels during puberty shows a direct correlation with this syndrome. Studies have suggested that high levels of androgens in a mother with PCOS can potentially move across the placenta and serve as a risk factor for causing the syndrome in the fetus. A number of findings have suggested that genetic factors may also be responsible for the transmission of polycystic ovarian syndrome among women of the same family. Recent studies have shown that daughters of women with PCOS might have increased ovarian volume even quite before the onset of puberty, thus supporting genetic correlations. The syndrome may also develop due to a number of prenatal and postnatal factors. Although its pathogenesis may begin in early fetal life the syndrome may manifest itself as early as childhood or adolescence.

\section{LITERATURE REVIEW AND DISCUSSION}

\section{The Clinical Problem}

Polycystic ovarian syndrome or PCOS may manifest itself through the occurrence of a number of features like hyperandrogenism, loss of ovarian functions, polycystic ovarian morphological features 
(Kousta, Tolis \& Franks, 2005). As mentioned earlier, environmental factors and changes in living pattern have contributed to increased obesity and occurrence of polycystic ovarian syndrome in adolescent girls. This is closely associated with ovulatory infertility which mainly causes PCOS. Abnormality in follicular development, rapid gonadotropin-releasing hormone pulses, excess release of luteinizing hormone and low levels of follicle stimulating hormone secretion contribute to abnormalities in ovulation and excess production of androgen. Hirsutism is another manifestation of excess androgen release. Cardiometabolic abnormalities and high risk of cardiovascular diseases are found to be associated with PCOS. The syndrome is also found to be related with reduced glucose tolerance, diabetes mellitus and higher triglyceride levels. Women suffering from PCOS have high risk of vascular diseases, high triglyceride and LDL cholesterol levels in blood which might not be dependent on BMI. (Wild et al., 2011). Women with a family history of the syndrome and who have undergone menopause have been found to be at a higher risk of cardiovascular events. However, much more research needs to be done to support this. High risk of endometrial cancer as much as about $9 \%$ have been found among women with this syndrome (Dumesic \& Lobo, 2013; Jakimiuk \& Issat, 2010). Obesity, improper ovulation and insulin resistance have been found to be high risk factors for the occurrence of endometrial cancer among women with PCOS. The occurrence of polycystic ovarian syndrome also increases the risks of pregnancy complications, apnea, emotional and sleep problems in patients.

\section{Diagnosis}

The pathological effects of polycystic ovarian syndrome in women are reported to involve various combinations of hyperandrogenism, improper ovulation, polycystic ovarian morphologic features and pregnancy loss (Balakrishnan, 2018).

\section{Hyperandrogenism}

High levels of androgen or hyperandrogenism, as it is called, has been found to be closely associated with the occurrence of polycystic ovarian syndrome. It may be manifested clinically with hirsutism and acne or it may be manifested biochemically with elevated serum androgen levels or may manifest in both ways. Women of different descents or origin may exhibit different degrees of hyperandrogenism. The degree of hyperandrogenism is generally assessed by visual grading of hair growth at nine areas of the body which are androgen- sensitive. Hyperandrogenism is dependent on the total testosterone levels which are measured by mass spectrometry. However, measurements of total testosterone, calculation of free testosterone levels and sex hormone binding globulin might give more accurate results (Rykova, 2016).

\section{Ovulatory dysfunction}

Women suffering from polycystic ovarian syndrome have been found to have higher cardiometabolic risk than those with either improper ovulation for polycystic ovaries. Unpredictable menses which occur at irregular intervals are typically associated with ovulatory dysfunction. The morphological features currently associated with PCOS involve 12 or more antral follicles in each ovary and a volume of more than $10 \mathrm{ml}$ in each ovary. Although ovarian ultrasonography is the major diagnostic tool to identify polycystic ovaries, however, it may not be required for diagnosis in cases where both hyperandrogenism and ovarian dysfunction are found. Many a times PCOS manifests itself in adolescence girls with the occurrence of irregular menses and emergence of hirsutism. However, sometimes some symptoms of this syndrome overlap with those of normal puberty. The symptoms of PCOS are more serious in women who have undergone menopause or are about to undergo it. Reports have suggested that a presumptive diagnosis of PCOS might have an earlier history of hyperandrogenism and oligomenorrhea. Studies suggest that patients suffering from PCOS should be screened for cardiometabolic risk factors through longitudinal screening. BMI measurement, measurement of waist circumference and blood pressure at regular intervals are included under such screening processes. PCOS patients should also be screened for impaired glucose tolerance, type 2 diabetes mellitus, smoking, sleep distress, depression and apnea. 


\section{CONCLUSION}

Polycystic ovarian syndrome (PCOS) is a metabolic disorder characterized by multiple symptoms including hormonal imbalances, hyperandrogenism, irregular menses, improper ovulation, pregnancy-loss which are all reflected in the mental health of women causing depression, distress, sleep and emotional problems. Maintenance of optimum body weight can reduce insulin and androgen levels and may restore regular ovulation. Since high-carbohydrate diets can lead to an increase in insulin levels, women suffering from PCOS should limit carbohydrates uptake and also consume lowfat diet.In addition, increased physical activity and regular exercise may also help in maintaining proper hormone levels and lowering blood sugar levels. Thus, adoption of a healthy lifestyle may help to improve the quality of life in PCOS patients.

\section{ACKNOWLEDGEMENT}

The authors express their sincere thanks to Department of Biotechnology, Government of India for the funding from DBT-STAR College grant, under which this review project was conducted. We are also grateful to the Principal and the DBT-STAR Coordinator, Surendranath College, for their support and encouragement in implementing this review project at undergraduate level.

\section{REFERENCES}

Balakrishnan, D. (2018). Polycystic Ovarian Syndrome and Early Pregnancy Loss. Journal of Medical Science and Clinical Research, 6(5).

Dumesic, D. A., \& Lobo, R. A. (2013). Cancer risk and PCOS. Steroids, 78(8), 782-785.

Jakimiuk, A., \& Issat, T. (2010). PCOS and cancer risk. Folia Histochemica Et Cytobiologica, 47(5).

Kousta, E., Tolis, G., \& Franks, S. (2005). Polycystic ovary syndrome. Revised diagnostic criteria and long-term health consequences. HORMONES-ATHENS-, 4(3), 133.

Rykova, O. (2016). Polycystic Ovary Syndrome: Laboratory Diagnosis of Hyperandrogenic Status of a Woman (A Review of the Article «The Polycystic Ovary Syndrome: a Position Statement from the European Society of Endocrinology», October 2014). International Journal of Endocrinology (Ukraine), (8.72), 69-71.

Varghese, B., Thomas, A., \& Sindhuja, B. (2015). Long Term Health Consequences of Polycystic Ovarian Syndrome. Journal of Pharmaceutical Research, 14, 75.

Wild, R. A., Rizzo, M., Clifton, S., \& Carmina, E. (2011). Lipid levels in polycystic ovary syndrome: systematic review and meta-analysis. Fertility and sterility, 95(3), 1073-1079. 


\title{
A Review on Curcumin and its Derivatives as Anticancer Agents
}

\author{
Subhadeep Sengupta, Suman Mahato, Jagannath Dalui, Lalita Das* \\ Department of Chemistry, Surendranath College, Kolkata, India \\ ${ }^{*}$ Corresponding Author's Email: das.lalita1@gmail.com
}

\begin{abstract}
Cancer is becoming one of the leading causes of death around the world. Really cancer is one of the most crucial disease for health hazards. In respect of the great advancement of cancer treatment, the incident and rate of mortality of cancer continue elevated. So, the search for further expert and less poisonous drug is still important of research of current aspects. Curcumin, the active ingredient in the Curcuma longa plant, has been widely used as an antioxidant, anti-cancer, and anti-inflammatory medicine for the past two decades. In the chapter, we will try to explain a summary of the medicinal importance as well as pharmacological action of curcumin and its subordinate in terms of activity of anticancer, and main mechanism of action. The literature values from the experimental and medical appraisal of curcumin in animal models, cancer cell lines, and human bodies have been used to set cellular level targets.
\end{abstract}

\section{Keywords: Anticancer Agents; Curcumin; Pathway of Cell; Mechanism for Action; System of Delivery}

\section{INTRODUCTION}

Cancer is one of the most life-threatening illnesses and major public health issues in the globe. In spite of great advancement of cancer treatment, the rate of occurrence of the illness and the mortality have not fallen (Gupta et al., 2017). In connection to the preparation of chemically agents of anticancer, we collect various anticancer agents with different effectiveness from plant origin like Taxus brevifolia, Catharanthus roseus, Betula alba, Cephalotaxus species, Curcuma longa, Erythroxylum previllei, and many others (Gupta et al., 2013).No other element is as significant as curcumin which is a pure crystalline form, collected from the rhizomes of Curcuma longa $L$ (turmeric) (Alibeiki et al., 2017) and was turned out from turmeric plant in a crystalline form which is pure. (Goel, Kunnumakkara \& Aggarwal, 2008). The bio-functional properties of curcumin and its derivatives are anti-tumour, antioxidant, and anti-inflammatory activities (Nagahama et al., 2016). Curcumin and its derivatives have gotten a lot of interest in the last two decades because of this. The characteristics are imposed to the main elements in curcumin (Aggarwal, Deb \& Prasad, 2015). Therefore, scientific research work has enlightened the structure activity relationship studies to improve biological and elemental properties of it.

The important machinery of role of curcumin shows its identical anticancer action which includes apoptosis inducing and proliferation of inhibiting the tumours by introducing a diversity of cellular signalling pathways (Kunnumakkara et al., 2017). Various studies indicated its antitumor activity on cancer in squamous cell of head and neck, breast, lung (Anand et al., 2008), showing its ability to kill multiple cancerous cells. Including all the advantages, its engagement is restricted due to its poor solubility in water and weak bioavailability in oral and chemical stability is low. The poor cellular uptake of it is another barrier. Because to its hydrophobic nature, it tends to enter the cell curtain and binds to the fatty acyl chains of lipids in membrane through $\mathrm{H}$-binding, which in term causes low appearance of curcumin within the cytoplasm (Barry et al., 2009). For beating these difficulties and betterment of the overall anticancer action of curcumin, different structural interchanges have been done to increase specific toxicology on definite cancer cells (Tsukamoto et al., 2014), to increase stability of bioavailability (Agrawal \& Gupta, 2000). Another presentation to use various systems to deliver is to increase 
properties like physiochemical and activity of treatment of curcumin. This write up emphasis on the modern articles of the SAR of the drug and its similar structure drugs and their activity cancer treatment in various cancer cell lines, models of animal, and clinical studies for human as well as numerous varieties of delivery systems of curcumin that have been applied for treatment of carcinoma.

\section{Structure of Curcumin and its derivatives:}

Alternation of chemical formula of curcumin does not only move the binding of receptor and active site of pharmacology of a drug but also changes properties of pharmacokinetics and physiochemical of it. Realizing the essential pharmacophoric point within a molecular structure, it requires an extensive conversation of its natural and synthetically made familiar type of compounds. Two phenyl rings associated with methoxyl and hydroxyl groups are attached and connected through a keto-enol linker (C7) of seven carbon atoms. A chemical interaction involving aryl-aldehydes and acetylacetone is typically reported to yield its derivatives. This association reaction produces several chemical analogues, eg, compounds with substitution of alkyl group at the middle carbon of the linkage at C7 moiety (Chen et al., 2006). According to a SAR investigation of curcumin derivatives, the presence of a hydrogen donor group in the same plane as the -diketone moiety is required for curcumin analogue antiandrogenic efficacy in the treatment of prostate cancer (Ohtsu et al., 2002). In addition, C7 atoms to C5-five carbon atom improves the antiandrogenic activity (Lin et al., 2006). As a result, several new curcumin compounds have been produced to introduce a methyl group at both $\mathrm{C} 2$ and $\mathrm{C} 6$ positions of the compounds. Methylation boosted the molecule's capacity to assault targets and activity, but it also increased its hydrophobicity significantly in comparison to curcumin, limiting its useable dose in cancer treatment (Qiu et al., 2010). Moreover, the stability of kinetically controlled prepared curcumin derivatives has shown the glycosylation of the pharmacophoric points. Aromatic ring actually improves solubility of the compound in water, which in turn enhances its kinetic stability and tends to a better response in therapy (Ferrari et al., 2009). The major ways of converting curcumin into a more excretable form during phase I and phase II metabolism include oxidation, reduction, and conjugation (glucuronidation and sulfurylation). Curcumin's hydroxyl groups $(4-\mathrm{OH})$ connected to the phenyl rings undergo conjugation processes. Curcumin's kinetic stability can be improved by masking the 4-OH groups (Cao et al. , 2014).

(A)

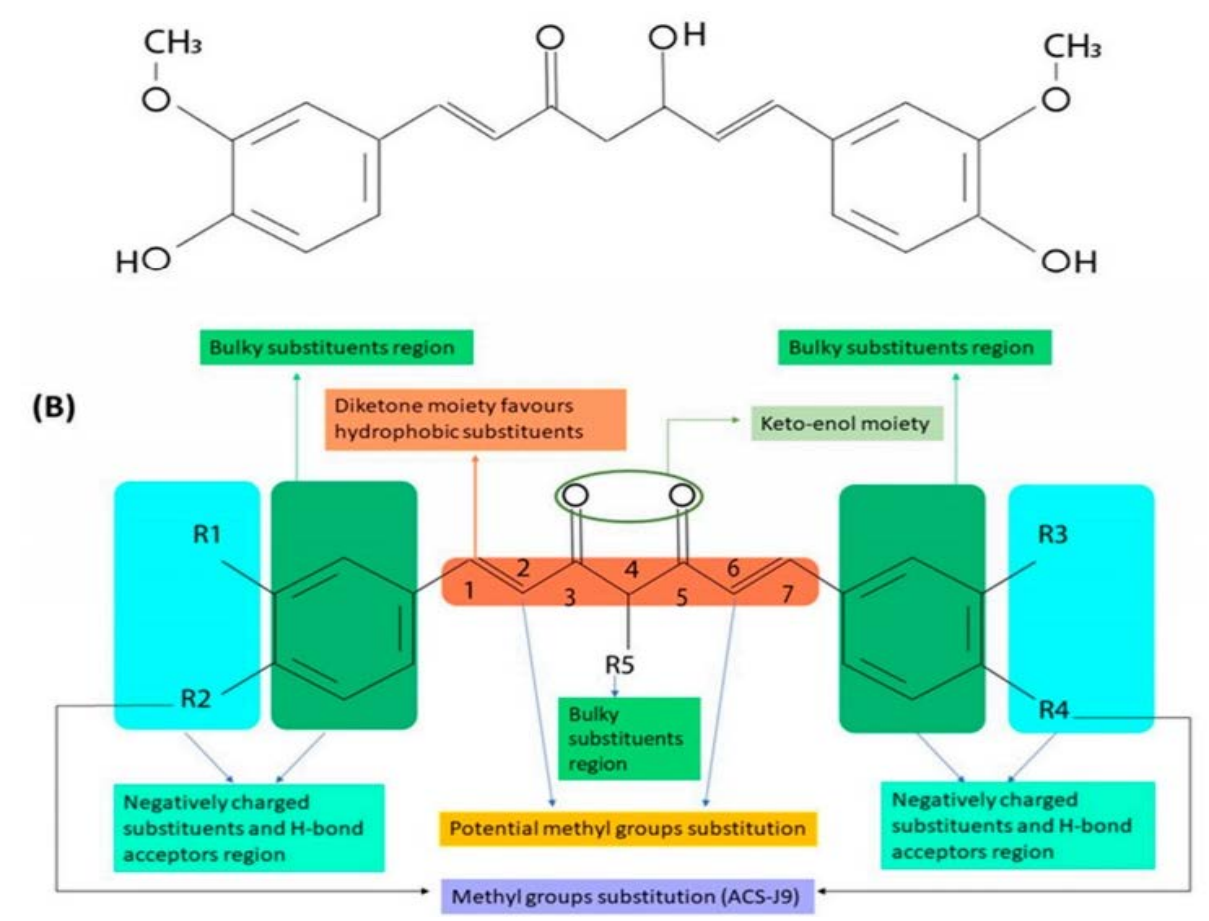

Figure 1: (A) Chemical sttucture of curcumin. (B) The main pharmacophores and potential substitution positions. Examples of current nanoparticle design strategies used to improve targeting. 


\section{Delivery systems of curcumin used in cancer therapy:}

To improve the feature and ability of target, curcumin has several delivery systems using different nanotechnologies. For the allowable different designs, different reasons should be considered to improve the efficiency and target of cellular level of the antimalignant agents. These reasons cover shape and size of the nanoparticles, properties of surface phenomenon, and nanoparticle which targets ligands. A brief summary of the delivery system of curcumin is discussed below:

A) Polymeric Nanoparticles: Different polymeric compounds have been prepared using nano formulations for drug delivery of curcumin for betterment of its biological activity (Sun, et al., 2008). In the delivery systems of drug, the bioconsistant and eco-friendly polymers are used due to low risk of toxic character (Naksuriya et al., 2014). Therefore, eco-friendly and synthetic polymer such as PLGA, poly (D, L-lactic-co-glycolic acid) and the polymers which are natural, such as, silk fibroin and chitosan widely used in delivery system as drug (Danhier et al., 2012). It is found that curcumin-PLGA was highly efficient as curcumin and it is found that at 15 -fold lower concentration, it inhibits mRNAs for inflammatory cytokines (CXCR3 and CXCL10) and increases cytokine interleukin-10 (IL-10) as anti-inflammatory agents in brain. In vivo study on rat shows that the bio sensitivity of curcumin-PLGA nanospheres increased 9-fold with respect to unprocessed curcumin when delivered with alkaloid compound, Piperine. When curcumin and piperine were combined, the activity of curcumin was increased by blocking hepatic and intestinal deactivation. (Shaikh, et al., 2009). The latter compound showed effective binding to cervical cancer cell but poor performance with respect to CUR-NPs (Punfa et al., 2012). PLGA which is spherical nanospheres are also delivered to dimethyl curcumin and tested in breast cancer cells. The PLGA nanospheres were able to redeem ASC-J9 by intra cellularly, giving to resistance of growth of estrogen-dependent MCF-7 cancer cells.

B) Liposomes: liposomes which is in nanoscale are outgoing and it is one of the most necessary medication delivery systems for anticancer agents. Recently in preparation of liposome an improved treatment for tumors which are drug-resistant and waned toxicity (Malam, Loizidou \& Seifalian, 2009). Because of its bilayer shell and watery interior, the liposome is an excellent transporter for both hydrophobic and hydrophilic molecules. Different liposome has been used to placing curcumin. Egg yolk phosphatidyl choline (EYPC), Dihexyl phosphate (DHP), and cholesterol digested curcumin make up the liposomal lipid doble layer. That method stabilized curcumin which is loaded proportionally to its content (Karewicz et al., 2011). Positively charged nanoliposomes was developed for delivery of curcumin by incorporating cationic polyethyleneimine (PEI) and polyethylene glycol (PEG) into formula. Though it has low encapsulation ability (45\%), this method has showed twenty-fold higher cytotoxic activity than redefined curcumin in various cell lines, which includes human A549 lung carcinoma, HepG2 hepatocellular carcinoma, HT29 colorectal carcinoma, and cervical carcinoma (Li et al., 2012) etc. In delivery of liposome in gene level, an important work led by Fujita et al. (2016) which used curcumin to monitor release of siRNA. When curcumin was incorporated into the liposomal formula, siRNA was released, showed a bell-shaped shape because of dose-dependent enhancement in permeability of liposome induced by curcumin.

c) Nanogels: Nanogels and hydrogels have got immense importance in the past few decades as an important delivery system of drug, very few studies have examined for treatment of cancer with delivery curcumin-nanogel. Recently synthetic as well as natural polymers are used to make the different polymeric hydrogel nanoparticles. Chitosan, chitin, alginate is the mostly examined natural polymers among the all, for the making of nanogels in delivery system of drug (Lee et al., 2014). The mostly used synthetic polymers are polyethylene oxide (PEO), polyvinyl alcohol (PVA), polyvinyl pyrrolidone (PVP), polyethyleneimine (PEI), and poly-N-isopropylacrylamide (PNIAA) (Hamidi, Azadi \& Rafiei, 2008). Main importance of natural hydrogels than synthetic ones are biodegradability and biocompatibility when they are used in drug delivery. Moreover, nanogels show unique features, which includes large area of surface for put a drug and a leaky structure for loading of drug and relief. 
For treatment of skin cancer, as a transdermal system was used with chitin nanogel and it is very toxic for human skin melanoma (A375) compare to human dermal fibroblast (HDF) cells without accommodating anticancer behaviour of curcumin (Mangalathillam et al., 2012). In a different study, a nanogel system which is hybrid and consists of a salt of alginic acid, pluronic and chitosan polymers can be prepared through the linked with a cross link of polycationic process and it was verified on HeLa cell line (Das, Kasoju \& Bora, 2010). This system of deliberation illustrated high skill of catching, and an important variety in rapid increase of cell was seen midway the cells which treated with unusual curcumin and curcumin-loaded hybrid nanogel.

d) Cyclodextrin complexes: Cyclodextrins are oligosaccharides which made of a lipophilic core and hydrophilic external layer. In delivery system of drug, these particular complexes give some advantageous properties, as for example, enhanced bioavailability, increased solubility, and better durability of the given drug. There are different types of cyclodextrins, such as natural ( $\alpha, \beta$, and $\gamma)$, polymerized, that have different solubility in water and have different relative molecular mass (Gidwani \& Vyas, 2015). Inclusion complexes and self-assembled cyclodextrins are examples of cyclic oligosaccharides complexes (Figure 2). Some literature reviews indicate that cyclodextrins can use as

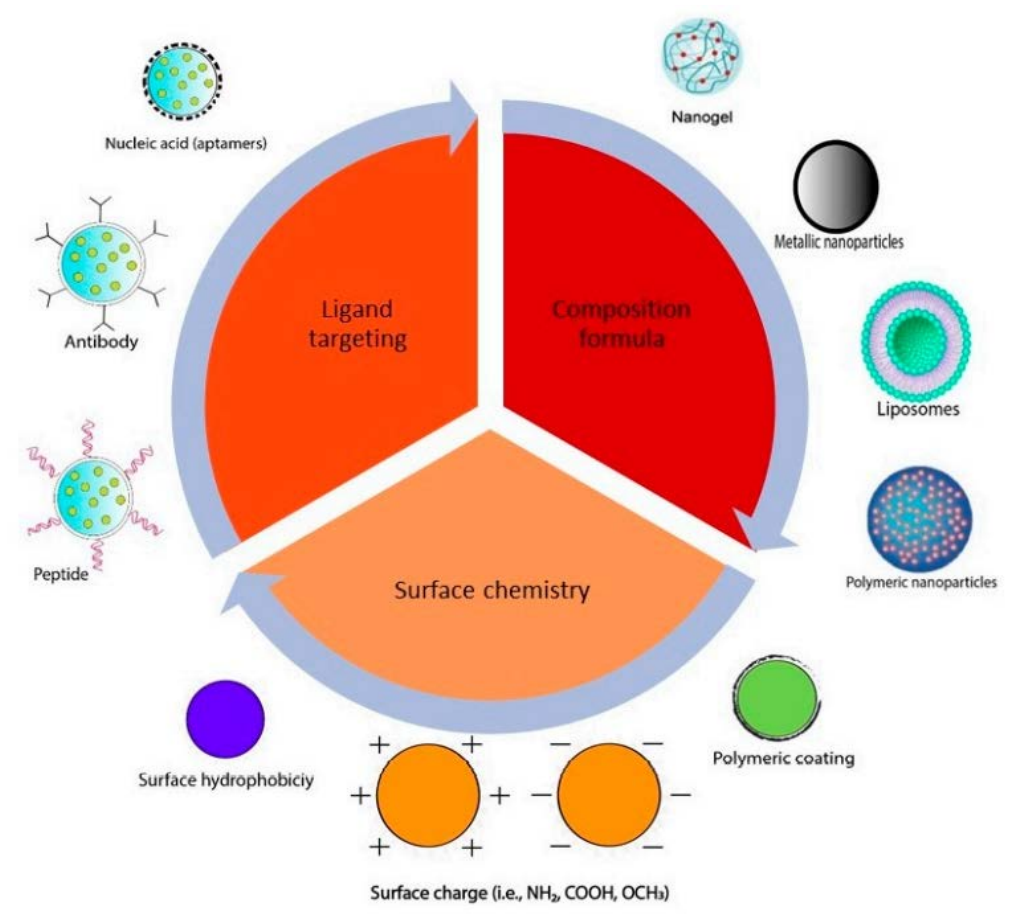

Figure 2: Examples of current nanoparticle design strategies used to improve targeting.

carriers in delivery of curcumin to minimize degradation, reduce nonselective toxicity and increase bioavailability. Self-assemble of $\beta$-cyclodextrin-curcumin showed that higher amount of curcumin was required by DU145 prostate cancer cells with respect to crude curcumin (Yallapu, Jaggi \& Chauhan, 2012) A supplementary salutary effect of curcumin-cyclodextrin complexes is found in case of lung cancer. When these complexes are injected to mice results in the reformed availability of curcumin and tumor size is reduced to a significant extend (Rocks et al., 2012).

\section{Anticancer activity of Curcumin:}

In vitro and in vivo studies: Curcumin shows significant results in controlling the growth of carcinoma cell and expansion in other cancer, such as cervical, intestine, pancreatic, breast, brain, head, and neck cancers. Next question is how curcumin and its derivatives work in different types of cancer from in vitro and in vivo studies in different cell lines of cancer and study of animal. 
Prostate Cancer: Curcumin has a strong efficiency to reduce expansion and introduce control cell death (Dorai, et al., 2001) in prostate cancer by intervening with a number of pathways of cell, which includes mitogen-activated protein kinase (MAPK), epidermal growth factor receptor (EGFR), and nuclear factor $K(N F K B)$. Recent study has expressed the efficiency of curcumin to activate protein kinase D1 (PKD1), gives rise to destruction of the oncogenic signalling pathway by $\beta$-catenin and MAPK and subsequently prohibition of prostate cancer (Sundram et al., 2012). Moreover, PKD1 was decrease from androgen-dependent to androgen-independent prostate cancer, and to change the activity and attack of cancer in prostate via interaction with E-cadherin (Mudduluru et al., 2011). Then, we can consider it as a new target for carcinoma in general and for prostate cancer specifically (LaValle et al., 2010).

Colorectal Cancer: Colorectal, is one of most important and malignant cancer. Patients having this cancer, they have to remove the tumor tissue in addition to chemotherapy (Carrato, 2008). Introduction of curcumin reduces in the cancer cells increasing levels of COX-2 protein (Garcea et al., 2005). Moreover, treatment of curcumin downregulates miR-21 gene, which is upregulation in cancer cells and prohibiting AP-1 (activator protein) binding to miR-21 gene. Treatment of HCT 116 carcinoma cells with curcumin results in arrest of cell cycle in the G2/M phase by gene regulation of miR-21 and inhibited the growth of tumor tissue. Another study has been done where curcumin enhances inhibition with respect to colon cancer cells which combines with ERRP, a pan-erb $B$ inhibitor (Xu etal., 2005).

Head and Neck Carcinoma: Neck or head cell carcinoma (HNSCC) is another dangerous cancer throughout the whole world, more than 30,000 cases were diagnosed every year (Vokes et al., 1993). Different neck and head cancer cell proved that it is able to forbid the growth of cell because of its consequence on different cellular pathways, mainly in a sharp increase in productivity of cell, eg., NF-KB and STAT3, which are stereotype in several head and neck carcinomas. Curcumin downregulates NF-kB and supresses interleukin-6 (IL-6)-mediated phosphorylation of STAT3, which inhibits the in a sharp increase in productivity of carcinoma cells.

Breast Cancer: Breast cancer is another deadly disease. It is one of the main cause of death in women (Ananthakrishnan, Balci \& Crowe, 2012). Besides therapy using radiation, lumpectomy, and therapy considering endocrine, the repetition of breast cancer rate is still high which depends on retrospective studies of meta-analysis (Houssami et al., 2012). Therefore, there is more requirement for efficient drugs and techniques. When curcumin was treated on a concentrationdependent manner, telomerase activity was shown. It was downregulated through hTERT by curcumin but not through the c-Myc mRNA pathway (Ramachandran et al., 2002). The effect of curcumin on regulatory proteins by cell-cycle, matrix metalloproteinases (MMPs), and NF-kB were evaluated in MDA-MB-231 and BT-483 breast cancer cell (Liu et al., 2015). The ability of curcumin to downregulate NF-B can be inferred from this study. It gives rise to a used or tending to inhibit cell growth. Though, a lowering of cyclic D1 in MDA-MB-231 cells and a lowering in CDK4 BT-483 were noticed when treated with curcumin.

Brain Cancer or glioblastoma: The brain cancer is increasing day by day. Glioblastoma (GBM) is one of the common malignant carcinomas in humans' brain. About $15 \%$ of all CNS tumors are added here. For treatment of GBM and brain tumors, therapy using radiation and surgical use are restricted for the passing of cancer cells into the healthy brain which leading to damage after treatment. Therefore, alternative methods which has lesser side effects are preferred than the conventional treatments. Curcumin has different sites for target molecule (Figure 3), thus, brain tumors has been cured through apoptosis, autophagy, angiogenesis, invasion, and metastasis. The rate-limiting stage in this process is the passage of the blood-brain barrier (BBB). Curcumin is one of the 
anticancer drugs that has a high rate of crossing the BBB barrier.

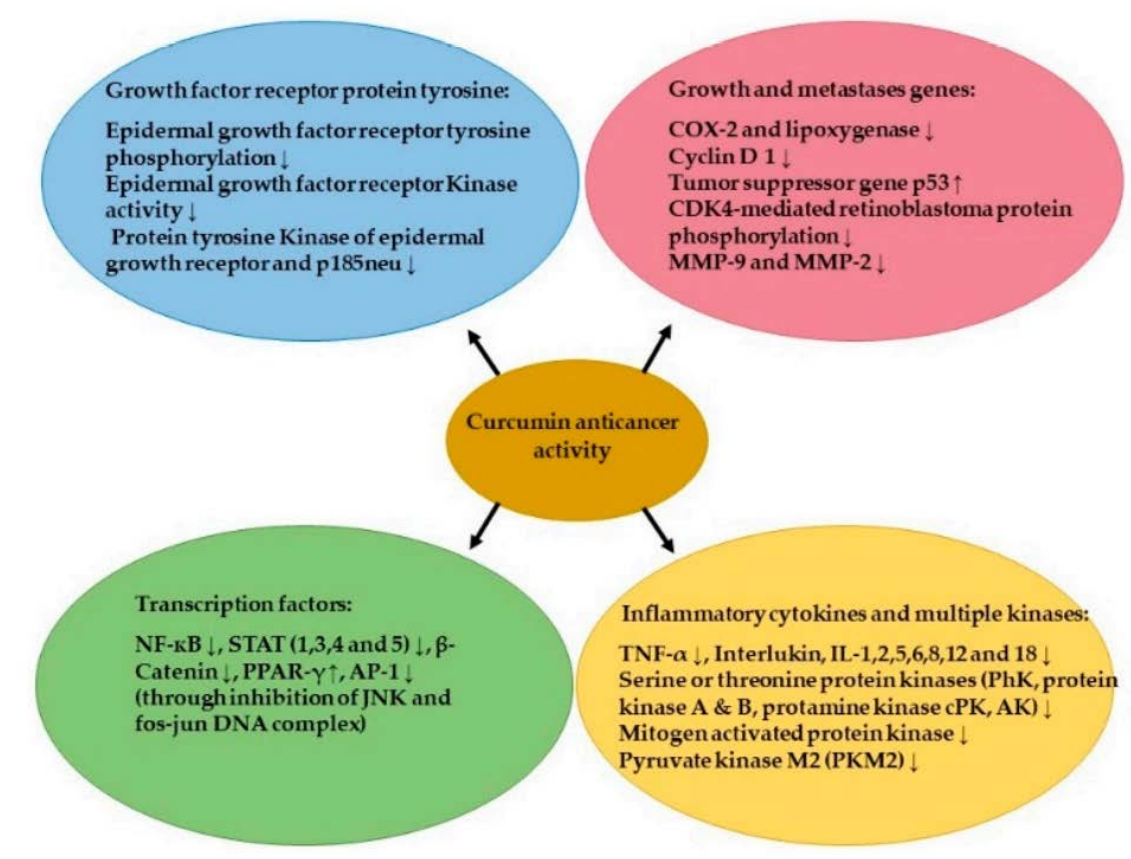

Figure 3: The Main Molecular Targets of Curcumin in Cancer Cells. $\uparrow:$ Increase; $\downarrow$ : Decrease; MMP: Matrix Metalloproteinase; AP-1: Activation Pro

\section{CONCLUSION}

The main element of the extract of Curcuma longa, Curcumin was discussed elaborately for the last few decades for its various important properties like antioxidant, anticancer, anti-inflammatory, and antiandrogenic effects. It shows various effects of anticancer against different types of carcinomas, like colorectal cancer, prostate cancer, breast cancer, pancreatic cancer, and head and neck cancer etc both in vivo and in vitro. Furthermore, Curcumin has been shown to be effective and safe in cancer patients when used alone or in conjunction with other anticancer medicines in humans. Curcumin is thought to exert anticancer effect through a variety of mechanisms, including interacting with numerous cellular pathways and activating or inhibiting the production of certain enzymes, cytokines, or growth factors. However, the anticarcinoma effect of curcumin is restricted due to mainly its low solubility in water, this results in reduced oral bioavailability and inadequate cellular absorption, and low stability in chemical. To overcome those difficulties, various approaches have been taken, such as structural alterations and using delivery systems of drug. The hydrogen donor group, the $\beta$-diketone moiety, the phenyl rings, and the substituent groups of Curcumin are the pharmacophoric points of it which contribute towards the biological activity of it. Chemical modification of these groups leads to the enhanced solubility or higher working efficiency of curcumin derivatives. Using various types of natural or synthetic polymers, lipids, or proteins, different delivery systems has been developed for delivery of curcumin to cancer cells. Some of them possess improved stability and cellular uptake of the drug, thus shows to a stronger anticarcinoma reaction. Despite the extreme effect to enhance the biological and physicochemical curcumins properties, there are still different reasons to be searched with respect to its availability, strength, and for the target cell specificity. It is found that the curcumin derivatives are not more efficient than curcumin itself. It is difficult for structural modification of Curcumin to reach a balance between efficiency and solubility.

\section{ACKNOWLEDGMENT}

The authors express their sincere thanks to Department of Biotechnology, Government of India for the funding from DBT-STAR College Grant, under which this review project was conducted. The authors are also grateful to the Principal, Surendranath College and the DBT-STAR Coordinator, Surendranath College, for their support and encouragement in implementing this review project. 


\section{REFERENCES}

Aggarwal, B. B., Deb, L., \& Prasad, S. (2015). Curcumin differs from tetrahydrocurcumin for molecular targets, signaling pathways and cellular responses. Molecules, 20(1), 185-205.

Agrawal, A. K., \& Gupta, C. M. (2000). Tuftsin-bearing liposomes in treatment of macrophage-based infections. Advanced Drug Delivery Reviews, 41(2), 135-146.

Alibeiki, F., Jafari, N., Karimi, M., \& Dogaheh, H. P. (2017). Potent anti-cancer effects of less polar Curcumin analogues on gastric adenocarcinoma and esophageal squamous cell carcinoma cells. Scientific Reports, 7(1), 1-9.

Anand, P., Sundaram, C., Jhurani, S., Kunnumakkara, A. B., \& Aggarwal, B. B. (2008). Curcumin and cancer: an "old-age" disease with an "age-old" solution. Cancer Letters, 267(1), 133-164.

Ananthakrishnan, P., Balci, F. L., \& Crowe, J. P. (2012). Optimizing surgical margins in breast conservation. International Journal of Surgical Oncology, 2012.

Barry, J., Fritz, M., Brender, J. R., Smith, P. E., Lee, D. K., \& Ramamoorthy, A. (2009). Determining the effects of lipophilic drugs on membrane structure by solid-state NMR spectroscopy: the case of the antioxidant curcumin. Journal of the American Chemical Society, 131(12), 4490-4498.

Cao, Y. K., Li, H. J., Song, Z. F., Li, Y., \& Huai, Q. Y. (2014). Synthesis and biological evaluation of novel curcuminoid derivatives. Molecules, 19(10), 16349-16372.

Carrato, A. (2008). Adjuvant treatment of colorectal cancer. Gastrointestinal Cancer Research: GCR, 2(4 Suppl 2), S42.

Chen, W. F., Deng, S. L., Zhou, B., Yang, L., \& Liu, Z. L. (2006). Curcumin and its analogues as potent inhibitors of low density lipoprotein oxidation: $\mathrm{H}$-atom abstraction from the phenolic groups and possible involvement of the 4-hydroxy-3-methoxyphenyl groups. Free Radical Biology and Medicine, 40(3), 526-535.

Danhier, F., Ansorena, E., Silva, J. M., Coco, R., Le Breton, A., \& Préat, V. (2012). PLGA-based nanoparticles: an overview of biomedical applications. Journal of Controlled Release, 161(2), 505522.

Das, R. K., Kasoju, N., \& Bora, U. (2010). Encapsulation of curcumin in alginate-chitosan-pluronic composite nanoparticles for delivery to cancer cells. Nanomedicine: Nanotechnology, Biology and Medicine, 6(1), 153-160.

Dorai, T., Cao, Y. C., Dorai, B., Buttyan, R., \& Katz, A. E. (2001). Therapeutic potential of curcumin in human prostate cancer. III. Curcumin inhibits proliferation, induces apoptosis, and inhibits angiogenesis of LNCaP prostate cancer cells in vivo. The Prostate, 47(4), 293-303.

Ferrari, E., Lazzari, S., Marverti, G., Pignedoli, F., Spagnolo, F., \& Saladini, M. (2009). Synthesis, cytotoxic and combined cDDP activity of new stable curcumin derivatives. Bioorganic \& Medicinal Chemistry, 17(8), 3043-3052.

Fujita, K., Hiramatsu, Y., Minematsu, H., Somiya, M., Kuroda, S. I., Seno, M., \& Hinuma, S. (2016). Release of siRNA from liposomes induced by curcumin. Journal of Nanotechnology, 2016.

Garcea, G., Berry, D. P., Jones, D. J., Singh, R., Dennison, A. R., Farmer, P. B., ... \& Gescher, A. J. (2005). Consumption of the putative chemopreventive agent curcumin by cancer patients: assessment of curcumin levels in the colorectum and their pharmacodynamic consequences. Cancer Epidemiology and Prevention Biomarkers, 14(1), 120-125.

Gidwani, B., \& Vyas, A. (2015). A comprehensive review on cyclodextrin-based carriers for delivery of chemotherapeutic cytotoxic anticancer drugs. BioMed Research International, 2015.

Goel, A., Kunnumakkara, A. B., \& Aggarwal, B. B. (2008). Curcumin as "Curecumin": from kitchen to clinic. Biochemical Pharmacology, 75(4), 787-809. 
Gupta, A. P., Khan, S., Manzoor, M. M., Yadav, A. K., Sharma, G., Anand, R., \& Gupta, S. (2017). Anticancer curcumin: natural analogues and structure-activity relationship. In Studies in Natural Products Chemistry (Vol. 54, pp. 355-401). Elsevier.

Gupta, A. P., Pandotra, P., Sharma, R., Kushwaha, M., \& Gupta, S. (2013). Marine resource: A promising future for anticancer drugs. In Studies in Natural Products Chemistry (Vol. 40, pp. 229-325). Elsevier.

Hamidi, M., Azadi, A., \& Rafiei, P. (2008). Hydrogel nanoparticles in drug delivery. Advanced Drug Delivery Reviews, 60(15), 1638-1649.

Houssami, N., Macaskill, P., von Minckwitz, G., Marinovich, M. L., \& Mamounas, E. (2012). Metaanalysis of the association of breast cancer subtype and pathologic complete response to neoadjuvant chemotherapy. European Journal of Cancer, 48(18), 3342-3354.

Karewicz, A., Bielska, D., Gzyl-Malcher, B., Kepczynski, M., Lach, R., \& Nowakowska, M. (2011). Interaction of curcumin with lipid monolayers and liposomal bilayers. Colloids and Surfaces B: Biointerfaces, 88(1), 231-239.

Kunnumakkara, A. B., Bordoloi, D., Padmavathi, G., Monisha, J., Roy, N. K., Prasad, S., \& Aggarwal, B. B. (2017). Curcumin, the golden nutraceutical: multitargeting for multiple chronic diseases. British Journal of Pharmacology, 174(11), 1325-1348.

LaValle, C. R., George, K. M., Sharlow, E. R., Lazo, J. S., Wipf, P., \& Wang, Q. J. (2010). Protein kinase D as a potential new target for cancer therapy. Biochimica et Biophysica Acta (BBA)-Reviews on Cancer, 1806(2), 183-192.

Lee, W. H., Loo, C. Y., Young, P. M., Traini, D., Mason, R. S., \& Rohanizadeh, R. (2014). Recent advances in curcumin nanoformulation for cancer therapy. Expert Opinion on Drug Delivery, 11(8), 1183-1201.

Li, X., Nan, K., Li, L., Zhang, Z., \& Chen, H. (2012). In vivo evaluation of curcumin nanoformulation loaded methoxy poly (ethylene glycol)-graft-chitosan composite film for wound healing application. Carbohydrate Polymers, 88(1), 84-90.

Lin, L., Shi, Q., Su, C. Y., Shih, C. C. Y., \& Lee, K. H. (2006). Antitumor agents 247. New 4ethoxycarbonylethyl curcumin analogs as potential antiandrogenic agents. Bioorganic \& Medicinal Chemistry, 14(8), 2527-2534.

Liu, B., Sun, L., Liu, Q., Gong, C., Yao, Y., Lv, X., ... \& Song, E. (2015). A cytoplasmic NF-kB interacting long noncoding RNA blocks IKB phosphorylation and suppresses breast cancer metastasis. Cancer Cell, 27(3), 370-381.

Malam, Y., Loizidou, M., \& Seifalian, A. M. (2009). Liposomes and nanoparticles: nanosized vehicles for drug delivery in cancer. Trends in Pharmacological Sciences, 30(11), 592-599.

Mangalathillam, S., Rejinold, N. S., Nair, A., Lakshmanan, V. K., Nair, S. V., \& Jayakumar, R. (2012). Curcumin loaded chitin nanogels for skin cancer treatment via the transdermal route. Nanoscale, 4(1), 239-250.

Mudduluru, G., George-William, J. N., Muppala, S., Asangani, I. A., Kumarswamy, R., Nelson, L. D., \& Allgayer, H. (2011). Curcumin regulates miR-21 expression and inhibits invasion and metastasis in colorectal cancer. Bioscience Reports, 31(3), 185-197.

Nagahama, K., Utsumi, T., Kumano, T., Maekawa, S., Oyama, N., \& Kawakami, J. (2016). Discovery of a new function of curcumin which enhances its anticancer therapeutic potency. Scientific Reports, $6(1), 1-14$.

Naksuriya, O., Okonogi, S., Schiffelers, R. M., \& Hennink, W. E. (2014). Curcumin nanoformulations: a review of pharmaceutical properties and preclinical studies and clinical data related to cancer treatment. Biomaterials, 35(10), 3365-3383. 
Ohtsu, H., Xiao, Z., Ishida, J., Nagai, M., Wang, H. K., Itokawa, H., ... \& Lee, K. H. (2002). Antitumor agents. 217. Curcumin analogues as novel androgen receptor antagonists with potential as antiprostate cancer agents. Journal of Medicinal Chemistry, 45(23), 5037-5042.

Punfa, W., Yodkeeree, S., Pitchakarn, P., Ampasavate, C., \& Limtrakul, P. (2012). Enhancement of cellular uptake and cytotoxicity of curcumin-loaded PLGA nanoparticles by conjugation with anti-Pglycoprotein in drug resistance cancer cells. Acta Pharmacologica Sinica, 33(6), 823-831.

Quu, X., Du, Y., Lou, B., Zuo, Y., Shao, W., Huo, Y., ... \& Bu, X. (2010). Synthesis and identification of new 4-arylidene curcumin analogues as potential anticancer agents targeting nuclear factor-KB signaling pathway. Journal of Medicinal Chemistry, 53(23), 8260-8273.

Ramachandran, C., Fonseca, H. B., Jhabvala, P., Escalon, E. A., \& Melnick, S. J. (2002). Curcumin inhibits telomerase activity through human telomerase reverse transcritpase in MCF-7 breast cancer cell line. Cancer Letters, 184(1), 1-6.

Rocks, N., Bekaert, S., Coia, I., Paulissen, G., Guéders, M., Evrard, B., ... \& Cataldo, D. (2012). Curcumin-cyclodextrin complexes potentiate gemcitabine effects in an orthotopic mouse model of lung cancer. British Journal of Cancer, 107(7), 1083-1092.

Shaikh, J., Ankola, D. D., Beniwal, V., Singh, D., \& Kumar, M. R. (2009). Nanoparticle encapsulation improves oral bioavailability of curcumin by at least 9-fold when compared to curcumin administered with piperine as absorption enhancer. European Journal of Pharmaceutical Sciences, 37(3-4), 223230.

Sun, M., Estrov, Z., Ji, Y., Coombes, K. R., Harris, D. H., \& Kurzrock, R. (2008). Curcumin (diferuloylmethane) alters the expression profiles of microRNAs in human pancreatic cancer cells. Molecular Cancer Therapeutics, 7(3), 464-473.

Sundram, V., Chauhan, S. C., Ebeling, M., \& Jaggi, M. (2012). Curcumin attenuates $\beta$-catenin signaling in prostate cancer cells through activation of protein kinase D1. PLoS one, 7(4), e35368.

Tsukamoto, M., Kuroda, K., Ramamoorthy, A., \& Yasuhara, K. (2014). Modulation of raft domains in a lipid bilayer by boundary-active curcumin. Chemical Communications, 50(26), 3427-3430.

Vokes, E. E., Weichselbaum, R. R., Lippman, S. M., \& Hong, W. K. (1993). Head and neck cancer. New England Journal of Medicine, 328(3), 184-194.

Xu, H., Yu, Y., Marciniak, D., Rishi, A. K., Sarkar, F. H., Kucuk, O., \& Majumdar, A. P. (2005). Epidermal growth factor receptor (EGFR)-related protein inhibits multiple members of the EGFR family in colon and breast cancer cells. Molecular Cancer Therapeutics, 4(3), 435-442.

Yallapu, M. M., Jaggi, M., \& Chauhan, S. C. (2012). Curcumin nanoformulations: a future nanomedicine for cancer. Drug Discovery Today, 17(1-2), 71-80. 


\section{Published by :}

Lincoln Research and Publications Limited, Australia in collaboration with

Lincoln University College, Malaysia

ISBN: 978-0-6488798-6-2

\section{www.lincolnrpl.org $\quad$ www.lucp.net}

